\title{
Non-Invasive Monitoring of Brain Tissue Temperature by Near-Infrared Spectroscopy
}

\author{
Veronica S. Hollis, M.Sci. \\ Department of Medical Physics and Bioengineering \\ University College London
}

Thesis submitted for the degree of Doctor of Philosophy (Ph.D.)

at the University of London

September 2002 
ProQuest Number: U643426

All rights reserved

INFORMATION TO ALL USERS

The quality of this reproduction is dependent upon the quality of the copy submitted.

In the unlikely event that the author did not send a complete manuscript and there are missing pages, these will be noted. Also, if material had to be removed, a note will indicate the deletion.

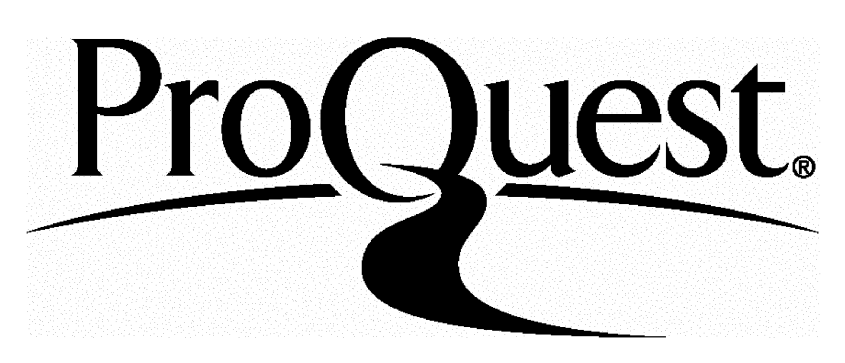

ProQuest U643426

Published by ProQuest LLC(2016). Copyright of the Dissertation is held by the Author.

All rights reserved.

This work is protected against unauthorized copying under Title 17, United States Code. Microform Edition (c) ProQuest LLC.

\author{
ProQuest LLC \\ 789 East Eisenhower Parkway \\ P.O. Box 1346 \\ Ann Arbor, MI 48106-1346
}




\section{Abstract}

Near-infrared spectroscopy (NIRS) is a non-invasive technique that is established as a research tool with which to study tissue oxygenation and haemodynamics, particularly in infant brains. In studies of oxygenation, NIRS relies on the oxygen-dependence of the haemoglobin spectrum to determine blood oxygen saturation. Similarly, the temperaturedependence of the tissue water absorption spectrum, due to changes in intermolecular hydrogen bonding, could act as an endogenous indicator of tissue temperature. This thesis describes the development of a methodology to determine in vivo tissue temperature using NIRS, based on the temperature response of the NIR water spectrum. One particular application of this technique is monitoring cerebral temperature in infants suffering from birth asphyxia, a condition in which the brain is deprived of oxygen. It has been shown that cooling the brain by a few degrees has a neuroprotective effect and the potential to prevent long-term damage. In order to assess the efficacy of mild hypothermia, brain temperature must be monitored continuously and non-invasively during the treatment, which is in principle possible with NIRS.

Experiments have been performed to accurately characterise the temperaturedependence (in the clinical range) of the NIR water absorption spectrum between 650 and $1050 \mathrm{~nm}$. Measurements of absorption are calibrated against temperature using a range of multivariate fitting techniques. In order to determine tissue temperature from the calibration of the water spectrum, the contribution of absorption to in vivo NIRS measurements must be separated from that of light scattering. Furthermore, absorption due to water must be extracted from the total absorption, which contains contributions from oxy- and deoxyhaemoglobin. A number of different approaches have been explored, including second derivative spectroscopy, non-linear diffusion theory modelling and spatially-resolved techniques. The performance of the tissue prediction algorithms are investigated using temperature-resolved measurements of a tissue phantom and ex vivo and in vivo tissues. 


\section{Acknowledgements}

Firstly I would like to thank my supervisors: Dr Mark Cope, for his inspirational guidance in the first year of my $\mathrm{PhD}$; Prof. David Delpy, for his excellent supervision throughout the course of my studies; Dr Clare Elwell, for her advice and discussions on clinically-related aspects of this project. I would also like to acknowledge Dr Roger Springett, to whom I am extremely grateful for all his time and effort spent helping me in my work with the CCD-spectrograph system, for which he developed the spectral acquisition software.

I would like to credit Dr Tiziano Binzoni (University of Geneva, Switzerland) for the in vivo measurements employed in much of the temperature prediction analysis, and to thank him for his enthusiastic communications regarding the work presented at the SPIE conference (San Jose, USA) in January 2001. I would also like to thank Prof. Eiji Okada (Keio University, Japan) for readily supplying the Monte Carlo simulations.

To my colleagues and friends at the Medical Physics Department, and those I have met this past year at the Wolfson Institute, thank you for the unconditional help, advice, and support. A special mention must go to the staff in the Medical Physics workshop, without whose technical expertise much of this work could not have taken place.

Thanks to all who have taken time to read sections of this thesis, especially Prof. Dave Delpy for his inexhaustible patience and thoroughness, and to Dr Jem Hebden and Prof. Andrew Todd-Pokpropek, whose feedback on the transfer document was invaluable.

The funding for this project was provided by the Engineering and Physical Sciences Research Council (EPSRC). I would like to express my gratitude to the Medical Physics Department for allocating me the award.

Finally, I want to thank my parents, my brother and sisters and all my friends for their love, understanding and encouragement, and in particular my partner Andrew, who has supported me in every possible way during my time in higher education. 


\section{Contents}

$\begin{array}{ll}\text { Abstract } & \mathbf{2}\end{array}$

$\begin{array}{ll}\text { Acknowledgements } & 3\end{array}$

$\begin{array}{ll}\text { Contents } & 4\end{array}$

$\begin{array}{lr}\text { List of Figures } & 8\end{array}$

$\begin{array}{ll}\text { List of Tables } & 14\end{array}$

$\begin{array}{ll}\text { Glossary } & 16\end{array}$

$\begin{array}{llr}1 & \text { Introduction } & 19\end{array}$

1.1 Objective and Motivation $\ldots \ldots \ldots \ldots \ldots \ldots \ldots$

1.2 Clinical Background $\ldots \ldots \ldots \ldots \ldots \ldots \ldots$

$1.2 .1 \quad$ Birth Asphyxia . . . . . . . . . . . . . . . 21

1.2.2 Delayed Energy Failure . . . . . . . . . . . . . . 22

1.2.3 Clinical Hypothermia . . . . . . . . . . . . . 23

1.3 Near-Infrared Spectroscopy $\ldots \ldots \ldots \ldots \ldots$

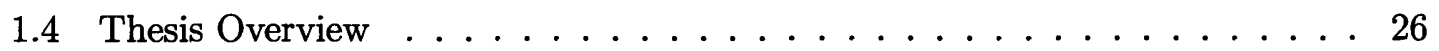

2 Light Transport in Biological Tissue $\quad 28$

2.1 Light Absorption . . . . . . . . . . . . . . . . . . . . 29

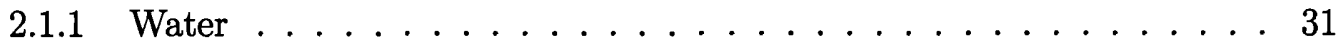

2.1 .2 Haemoglobin $\ldots \ldots \ldots \ldots \ldots \ldots \ldots$

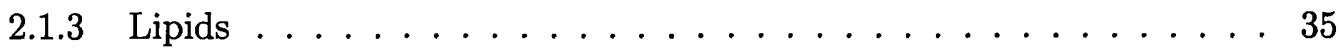




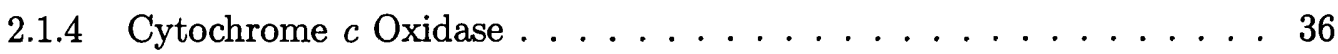

2.1.5 Other Chromophores . . . . . . . . . . . . . . 37

2.2 Light Scattering $\ldots \ldots \ldots \ldots \ldots \ldots \ldots$

2.2 .1 Scattering Theory $\ldots \ldots \ldots \ldots$

2.2.2 Light Scattering in Tissue . . . . . . . . . . . . . . . 42

2.3 Models of Light Propagation in Turbid Media . . . . . . . . . . . . . . 46

2.3.1 The Diffusion Approximation . . . . . . . . . . . . 47

2.3.2 The Monte Carlo Method . . . . . . . . . . . . . 51

3 Practical Aspects of Tissue NIRS $\quad 53$

3.1 Tissue NIRS Measurement Techniques . . . . . . . . . . . . . . . . . 54

3.1.1 Light Attenuation in Tissue . . . . . . . . . . . . . . . 54

3.1 .2 The Differential Pathlength . . . . . . . . . . . . 58

3.1.3 In Vivo Measurements . . . . . . . . . . . . . . 59

3.2 NIRS Steady-State Instrumentation $\ldots \ldots \ldots$. . . . . . . . . 60

3.3 Optical Coefficients in Whole Tissue . . . . . . . . . . . . . 63

3.3 .1 Skin . . . . . . . . . . . . . . 6 67

3.3 .2 Muscle .......................... 67

3.3 .3 Bone . . . . . . . . . . . . . . . . . 69

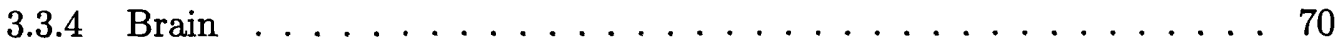

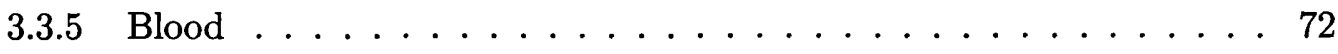

4 Methods of Tissue Temperature Measurement $\quad 73$

4.1 Measurement of Core Body Temperature . . . . . . . . . . . . . . . 74

4.1.1 Tympanic Membrane Thermometry . . . . . . . . . . . . 74

4.1.2 The Zero Heat Flow Method . . . . . . . . . . . . 76

4.2 Non-Invasive Brain Temperature Measurement $\ldots \ldots \ldots$. . . . . . . 79

4.2.1 Microwave Radiometry . . . . . . . . . . . . . . 79

4.2.2 Magnetic Resonance Thermometry . . . . . . . . . . . . 81

4.2 .3 Ultrasound Thermometry . . . . . . . . . . . . . . 82 
4.3 Tissue Temperature Monitoring by NIRS $\ldots \ldots \ldots . \ldots . \ldots$

4.3.1 Changes in Absorption with Temperature . . . . . . . . . 85

4.3.1.1 Water ................... 85

4.3.1.2 Haemoglobin . . . . . . . . . . . . . . 89

4.3.1.3 Whole Tissue . . . . . . . . . . . . . . 90

4.3.2 Changes in Scattering with Temperature: Whole tissue . . . . . . 93

4.3.3 Effect of Temperature on In Vivo NIRS Measurements . . . . . . . 97

5 Characterisation of the NIR Water Absorption Spectrum 99

5.1 Measuring the Temperature Response of the Water Absorption Spectrum . 100

5.1 .1 Experimental Design . . . . . . . . . . . . . . 100

5.1 .2 Experimental Methods and Results . . . . . . . . . . . . . . 102

5.1 .2 .1 Method I . . . . . . . . . . . . . . 103

5.1.2.2 Analysis and Results I . . . . . . . . . . 106

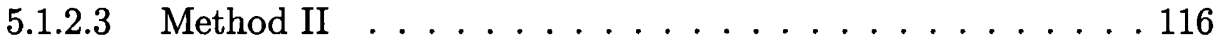

5.1.2.4 Analysis and Results II . . . . . . . . . . . . . 119

5.1.3 Comparison and Further Analysis of Results I and II . . . . . . . 121

5.2 Effects of Water Binding on the NIR Absorption Spectrum . . . . . . 124

5.2.1 Organic Solute: Sodium Carboxymethylcellulose . . . . . . . . . 124

5.2.1.1 Experimental Method . . . . . . . . . . . 125

5.2.1.2 Results and Discussion $\ldots \ldots \ldots \ldots \ldots$

5.2.2 Inorganic Solute: Sodium Chloride . . . . . . . . . . . 128

5.2.2.1 Experimental Method . . . . . . . . . . . . 128

5.2.2.2 Results and Discussion . . . . . . . . . . 128

5.3 Temperature Calibration of the NIR Water Absorption Spectrum . . . . 130

5.3.1 Gaussian Component Method . . . . . . . . . . . . . . . 131

5.3.2 Classical Least-Squares $\ldots \ldots$. . . . . . . . . . . 135

5.3 .3 Inverse Least-Squares $\ldots \ldots \ldots$. . . . . . . . . . 138

5.3 .4 Hybrid Method . . . . . . . . . . . . . . . 140

5.3.5 Principal Component Regression . . . . . . . . . . . . . . . 142 
5.3.5.1 Theory ....................... 142

$5.3 .5 .2 \quad \operatorname{Results} \ldots \ldots \ldots \ldots \ldots \ldots$

5.3.6 Partial Least Squares Regression . . . . . . . . . . . . . 149

5.3.6.1 Theory ....................... 149

5.3.6.2 Results . . . . . . . . . . . . . . 152

6 NIRS Measurements as a Function of Temperature 158

6.1 Chicken Muscle . . . . . . . . . . . . . . . . . . . 159

6.1.1 Experimental Design and Method . . . . . . . . . . 159

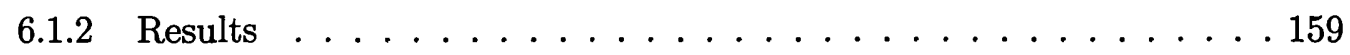

6.1 .3 Discussion . . . . . . . . . . . . . . . 161

6.2 Human Forearm . . . . . . . . . . . . . . . . . 164

6.2.1 Experimental Design and Method . . . . . . . . . . . 164

6.2 .2 Results and Discussion . . . . . . . . . . . 166

6.3 Tissue Phantom $\ldots \ldots \ldots \ldots \ldots$

6.3.1 Experimental Design and Method . . . . . . . . . . 169

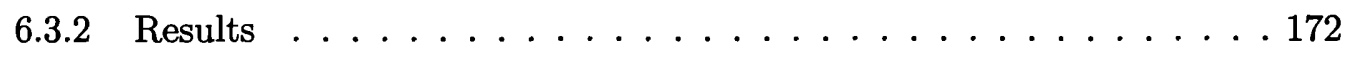

6.3 .3 Discussion . . . . . . . . . . . . . . . 174

7 NIRS Tissue Temperature Prediction Algorithms $\quad 177$

7.1 Algorithm I: Linear SDS Method . . . . . . . . . . . . . . 178

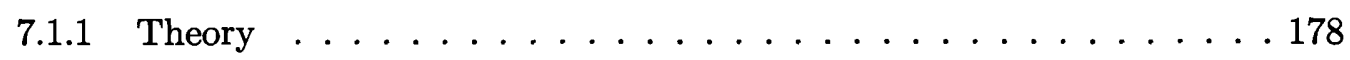

7.1 .2 The Savitzsky-Golay Filter . . . . . . . . . . . . 180

7.1.3 Determining Chromophore Concentrations by SDS . . . . . . . 181

7.1 .4 Temperature Prediction . . . . . . . . . . . . 186

7.2 Algorithm II: Non-Linear Optimisation Method . . . . . . . . . . . . . 190

7.2 .1 Theory $\ldots \ldots \ldots \ldots \ldots \ldots \ldots \ldots \ldots \ldots \ldots \ldots \ldots \ldots \ldots \ldots \ldots \ldots$

7.2.2 Application to in Vivo Tissue Data . . . . . . . . . . . . 195

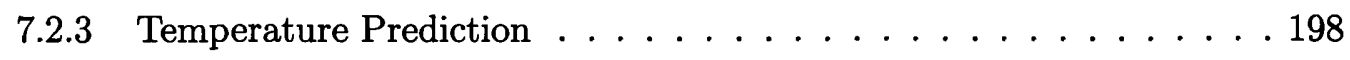

7.2 .4 Discussion . . . . . . . . . . . . . . . . 201 
7.3 Algorithm III: Spatially-Resolved Reflectance Method . . . . . . . . . 207

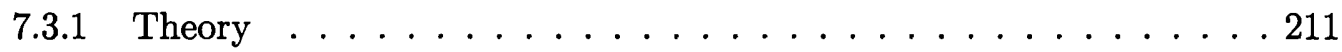

7.3.2 Application to Tissue Phantom Data . . . . . . . . . . . . . . . 214

7.3.3 Temperature Prediction . . . . . . . . . . . . 218

8 Conclusions $\quad 221$

8.1 Project Summary . . . . . . . . . . . . . . . . . . 222

8.1.1 Temperature-Dependence of the NIR Water Spectrum . . . . . . 222

8.1.2 Calibration of the Water Spectrum Against Temperature . . . . . 223

8.1.3 Temperature Prediction Algorithms . . . . . . . . . . . . 223

8.2 Further Work . . . . . . . . . . . . . . . . . . . . . . 224

A Error Analysis for Calculation of $\mu_{a \mathbf{T}} \quad \mathbf{2 2 8}$

A.1 Method I . . . . . . . . . . . . . . . . 230

A.2 Method II . . . . . . . . . . . . . . . . . 231

B Eigenvector Proof for PCA 233

C Effect of Water Concentration on PCR Temperature Prediction 235

$\begin{array}{ll}\text { D Orthogonality Proof for PLSR } & 238\end{array}$

$\begin{array}{ll}\text { References } & \mathbf{2 4 0}\end{array}$ 


\section{List of Figures}

1.1 Cross-sectional view of the (adult) cerebral hemispheres indicating the areas affected by HIE in new-born infants . . . . . . . . . . . . . . 21

1.2 Cooling cap applied to a new-born infant for the induction of mild hypothermia by selective head cooling . . . . . . . . . . . . . . 24

2.1 The absorption spectrum of pure water in the range $200-10,000 \mathrm{~nm} \quad \ldots \quad$. 32

2.2 Specific absorption spectra of $\mathrm{Hb}$ and $\mathrm{HbO}_{2}$ in the NIR from $650-1050 \mathrm{~nm} 34$

2.3 The absorption spectrum of pork fat (lipid) in the NIR from 800-1080 nm . 35

2.4 Specific difference absorption spectrum of cytochrome $c$ oxidase in the NIR from $650-970 \mathrm{~nm} \ldots \ldots \ldots \ldots \ldots \ldots$

2.5 A 'generalised' human cell . . . . . . . . . . . . . . . . . . . . . 42

3.1 The non-linear relationship between attenuation and absorption is approximately linear for small changes in attenuation . . . . . . . . . . 57

3.2 Schematic diagram of the generic equipment used for the CW spectroscopic measurements of this project $\ldots \ldots \ldots \ldots$. . . . . . . . 61

3.3 Three-dimensional view of the skin . . . . . . . . . . . . 67

3.4 Optical properties of ex vivo Caucasian dermis in the NIR . . . . . . . . . 68

3.5 Human skeletal muscle . . . . . . . . . . . . . . . . . . . . 68

3.6 Optical properties of ex vivo human abdominal muscle in the NIR . . . . . 69

3.7 Cross-section of flat bone in the human skull . . . . . . . . . . . . 69

3.8 Optical properties of ex vivo porcine skull in the NIR . . . . . . . . . . 70

3.9 A typical neurone present in the CNS $\ldots \ldots \ldots \ldots \ldots$

3.10 Optical properties of neonatal grey and white brain matter in the NIR . . 71

4.1 Schematic diagram of the deep body thermometer probe. . . . . . . . . 77 
4.2 The three normal modes of vibration of a water molecule in the liquid phase 85

5.1 Experimental set-up for the measurement of the NIR water absorption spectrum and its temperature response. . . . . . . . . . . . . . . 101

5.2 Definition of intensity measurements in determination of water absorption spectrum using Method I . . . . . . . . . . . . . . . . . . . . 109

5.3 NIR absorption coefficient in the region of the $740 \mathrm{~nm}$ water absorption band measured using Method I as a function of temperature between 44

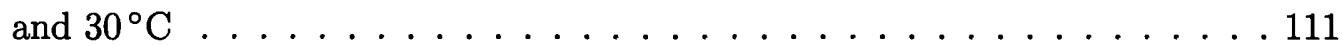

5.4 NIR absorption coefficient in the region of the $840 \mathrm{~nm}$ water absorption band measured using Method I as a function of temperature between 44

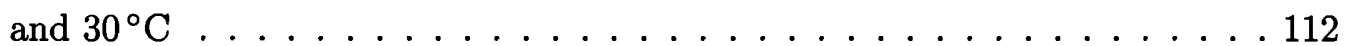

5.5 NIR absorption coefficient in the region of the $970 \mathrm{~nm}$ water absorption band measured using Method I as a function of temperature between 44

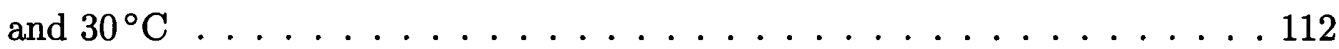

5.6 Close-up display of the water absorption spectrum in the range $895-920 \mathrm{~nm}$ measured using Method I . . . . . . . . . . . . . . . . . . 114

5.7 Attenuation through $1 \mathrm{~cm}$ of silicone oil in the range $700-950 \mathrm{~nm} . \ldots 115$

5.8 Second derivative comparison of measurements of water absorption with and without oil layer in the range $700-915 \mathrm{~nm} \ldots \ldots . \ldots 115$

5.9 NIR absorption coefficient in the region of the 740 and $840 \mathrm{~nm}$ water absorption bands measured using Method II as a function of temperature between

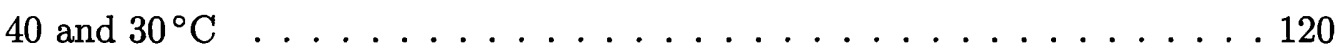

5.10 NIR absorption coefficient in the region of the $970 \mathrm{~nm}$ water absorption band measured using Method II as a function of temperature between 41

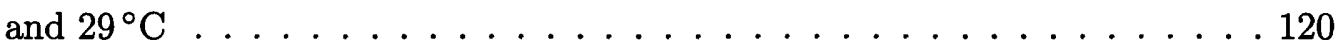

5.11 Temperature coefficient of the water absorption coefficient in the range 650$920 \mathrm{~nm}$ and $900-1050 \mathrm{~nm} \ldots \ldots . \ldots \ldots \ldots$

5.12 Absorption coefficient of CMC solutions at concentrations $0.1,0.2$ and $0.3 \%$

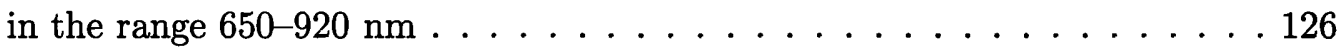

5.13 Absorption coefficient of CMC solutions at concentrations $0.1,0.2$ and $0.3 \%$

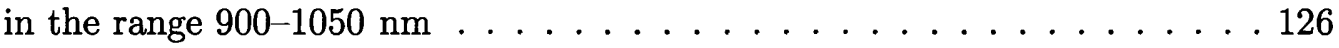


5.14 Absorption coefficient of isotonic saline compared to pure water at $30^{\circ} \mathrm{C}$ in the ranges $650-925$ and $900-1050 \mathrm{~nm} \ldots \ldots 129$

5.15 Temperature coefficients of isotonic saline and pure water absorption spectra in the ranges $650-925$ and $900-1050 \mathrm{~nm} \ldots \ldots 130$

5.16 Decomposition of the NIR water absorption spectrum measured at $42^{\circ} \mathrm{C}$ in the range $650-1050 \mathrm{~nm}$ into 12 Gaussian components . . . . . . . . . . . 133

5.17 Estimated against measured temperature for the CLS temperature calibration of the three NIR water absorption bands . . . . . . . . . 136

5.18 Temperature residuals for the CLS calibration of the NIR water absorption

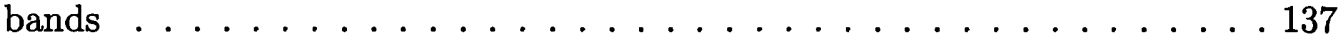

5.19 Temperature residuals for the 'hybrid' calibration of the NIR water absorption bands . . . . . . . . . . . . . . . . . . . . . . . 141

5.20 A graphical representation of PCA in the case of two independent variables 143

5.21 Cumulative percentage of the total variance in the water absorption spectra for the first four PCs . . . . . . . . . . . . . . . . . . . 145

5.22 The first and second loadings of the three NIR water absorption bands measured as a function of temperature, as determined by PCA . . . . 146

5.23 Temperature residuals for PCR calibration with 2 and 3 PCs of the three NIR water absorption bands . . . . . . . . . . . . . . 147

5.24 Comparison of first four weight and loading vectors from the PLSR temperature calibration of the water absorption spectra $\ldots \ldots \ldots 4$

5.25 The first four loading vectors from the PCR temperature calibration of the water absorption spectra . . . . . . . . . . . . . . . 155

5.26 Comparison of the temperature residuals determined from the PCR and PLSR calibrations of the water absorption spectra with 3 and 4 components 156

6.1 Relative changes in attenuation with heating of chicken muscle tissue. . . . 160

6.2 Difference attenuation during cooling of chicken muscle tissue . . . . . . 161

6.3 Experimental set-up for in vivo measurements of the adult forearm as a

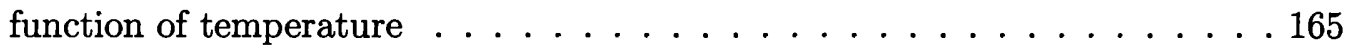

6.4 Attenuation spectra measured during cooling of the adult forearm . . . . 166 
6.5 Final difference attenuation spectrum of the adult forearm cooled from 37

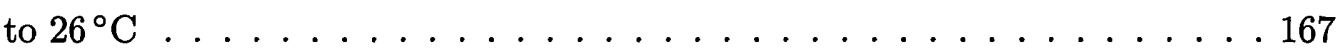

6.6 The specific absorption spectrum of copper sulphate in the region $650-925$

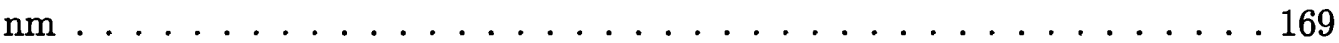

6.7 Spatially-resolved attenuation spectra of tissue phantom measured at $40.8^{\circ} \mathrm{C}$ using multi-distance fibre with source-detector separations of 1.8 , .

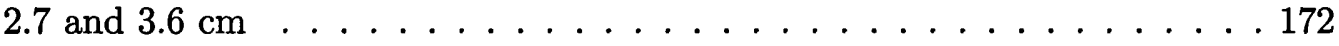

6.8 Absorption spectrum of soybean oil (constituent of Intralipid-10\% ${ }^{\mathrm{TM}}$ ) measured at $37^{\circ} \mathrm{C}$ in the range $650-925 \mathrm{~nm} \ldots \ldots \ldots 173$

6.9 Final difference attenuation spectra of spatially-resolved phantom measurements over the temperature range $40.8-33.8{ }^{\circ} \mathrm{C} \ldots \ldots 173$

6.10 The absorption and transport scattering spectra of the tissue phantom in the range $650-925 \mathrm{~nm}$, estimated for a temperature of $37^{\circ} \mathrm{C} \ldots \ldots 174$

7.1 The SDS approximation used to determine tissue chromophore concentrations from NIRS measurements of attenuation . . . . . . . . . . . 179

7.2 Data smoothing by the Savitzsky-Golay filter . . . . . . . . . . 180

7.3 Second derivative spectra of human forearm attenuation and tissue chromophore absorption spectra in the range $690-980 \mathrm{~nm} \ldots \ldots . \ldots 181$

7.4 WDPL corrections in the range $720-880 \mathrm{~nm}$ used in the estimation of chromophore concentrations by SDS . . . . . . . . . . . 186

7.5 Predicted against measured temperature using the SDS method on a simulated tissue attenuation data set . . . . . . . . . . . . 189

7.6 Graphical demonstration of the Newton-Raphson root-finding method . . . 192

7.7 Percentage errors in the difference absorption determined by the NewtonRaphson optimisation routine as a function of the ratio of input to actual $\mu_{a c a l}$ and $\mu_{s}^{\prime}$ values . . . . . . . . . . . . . . . . 194

7.8 Calibration absorption and transport scattering spectra used in the reconstruction of the absorption differences from measurements of attenuation on the adult forearm using the Newton-Raphson optimisation routine . . 197

7.9 Final difference absorption spectra reconstructed from difference attenuation measurements of the adult forearm using the Newton-Raphson optimisation routine . . . . . . . . . . . . . . . . . 198 
7.10 Chromophore concentrations as a function of temperature determined by a linear fit of the chromophore spectra to the reconstructed difference absorption spectra . . . . . . . . . . . . . . . . . . 199

7.11 Predicted against measured tissue temperature as determined by applying the PCR calibration of the water spectrum to the reconstructed tissue absorption spectra . . . . . . . . . . . . . . . . . 201

7.12 The effect of underestimating the change in scattering upon the reconstructed difference absorption . . . . . . . . . . . . . . . 202

7.13 Final difference absorption spectra reconstructed from difference attenuation measurements of the adult forearm incorporating simulated changes in the transport scattering spectrum with temperature $\ldots \ldots 203$

7.14 Predicted against measured tissue temperature for absorption reconstructions incorporating a decrease in the transport scattering coefficient with temperature . . . . . . . . . . . . . . . . . . 204

7.15 Temperature residuals for reconstructions using Set I and Set II with Coefficient II . . . . . . . . . . . . . . . . . . 205

7.16 Log normalised spatially-resolved reflectance generated using diffusion theory, Monte Carlo simulations and experimental measurements of a tissue phantom, at three source-detector separations between 1.8 and $3.6 \mathrm{~cm} \ldots 209$

7.17 Percentage error of the linear approximation of spatially-resolved intensity compared to the exact diffusion theory solution as a function of sourcedetector separation in the range $1.8-3.6 \mathrm{~cm} \ldots 212$

7.18 Absorption and transport scattering coefficients of the tissue phantom in the range 650-925 $\mathrm{nm}$ reconstructed using the SRA method . . . . . . . . 215

7.19 Effect of phantom temperature in the range $40.8-33.8^{\circ} \mathrm{C}$ on the slope and offset of the SRA approximation at $850 \mathrm{~nm} \ldots \ldots 216$

7.20 Reconstructed absorption and transport scattering coefficients of the tissue phantom at $850 \mathrm{~nm}$ as a function of temperature $\ldots \ldots \ldots 217$

7.21 Second derivative regression coefficients of saline and copper sulphate components as a function of temperature for a source-detector separation of 1.8

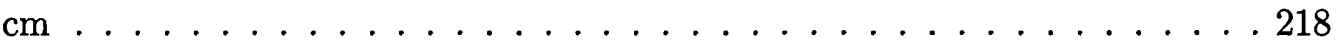


7.22 Tissue phantom temperature predicted from reconstructions of $\mu_{a}$ using the SRA method for the inclusion of one PLSR calibration factor . . . . . . . 219

8.1 Cross-section of a PMDF calculated for absorption in a 2D model of the neonatal head as a function of depth into the head from the surface $\ldots 226$ 


\section{List of Tables}

3.1 NIR optical coefficients of ex vivo tissues. . . . . . . . . . . . . . 65

3.2 NIR optical coefficients of in vivo tissues . . . . . . . . . . . . . . . 66

5.1 Experimental and acquisition parameters used during the measurement of water absorption by Method I . . . . . . . . . . . . . . . 105

5.2 Experimental and acquisition parameters used during the measurement of water absorption by Method II . . . . . . . . . . . . . 118

5.3 Main differences between Methods I and II for the measurement of the water absorption spectrum . . . . . . . . . . . . . . 121

5.4 Percentage errors in $\mu_{a \mathrm{~T}}$ determined for both Method I and II . . . . 123

5.5 Coefficients obtained from the linear regression of the amplitudes and widths of the 12 Gaussian components against water temperature . . . . . 134

5.6 Statistical parameters for the CLS temperature calibration of the three NIR water absorption bands . . . . . . . . . . . . . . 137

5.7 Statistical parameters for the 'hybrid' temperature calibration of the three NIR water bands . . . . . . . . . . . . . . . . . . . . . 141

5.8 Statistical parameters for the PCR calibration of the NIR water bands . . . 146

5.9 Comparison of temperature calibration parameters obtained from the application of PCR and PLSR to temperature-dependent NIR water absorption spectra . . . . . . . . . . . . . . . . . 153

5.10 Comparison of temperature prediction parameters obtained from the application of PCR and PLSR to the NIR water spectra using the cross-validation

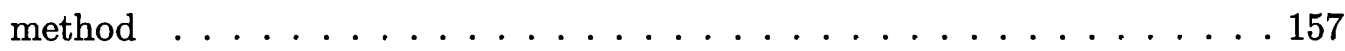

7.1 SDS-estimated chromophore concentrations using simulated forearm attenuation spectra and WDPL-corrected chromophore absorption spectra . . . . 184 
7.2 The effect of different WDPL corrections of the chromophore spectra on the SDS-estimated chromophore concentrations of a simulated forearm attenuation spectrum . . . . . . . . . . . . . . . 185

7.3 Statistical parameters for the PCR calibration of the temperature-matched ranges of the water absorption spectrum measured by Method I . . . . . 187

7.4 Standard error for water temperature prediction using the SDS method, applied to the measured water absorption spectra . . . . . . . . . 188

7.5 Optical coefficients used in Monte Carlo simulations of diffuse reflectance from a semi-infinite medium . . . . . . . . . . . . . . . . . 193

7.6 SDS-estimated chromophore concentrations of the flrst 50 attenuation spectra measured on the adult forearm . . . . . . . . . . . 196

7.7 Prediction of the optical coefficients used to generate Monte Carlo simulations of spatially-resolved reflectance by the SRA method $\ldots \ldots$. . . . 214

C.1 Statistical parameters for the PCR calibration of the temperature-matched ranges of the water absorption spectrum measured by Method I . . . . . . 237 


\section{Glossary}

ADC

ADP

ATP

CCD

CLS

CNS

CSF

CW

DBT

DP

DPF

FWHM

$g$

$\mathrm{Hb}$

$\mathrm{HbO}_{2}$

HIE

ILS

ISF

ITT

$m$

$\mu_{a}$

$\mathrm{MC}$

MLR

MRI

MRS

$\mu_{s}$

$\mu_{s}^{\prime}$
Analogue-to-Digital Converter

Adenosine Diphosphate

Adenosine Triphosphate

Charged-Coupled Device

Classical Least Squares

Central Nervous System

Cerebrospinal Fluid

Continuous Wave

Deep Body Thermometer

Differential Pathlength

Differential Pathlength Factor

Full-Width Half-Maximum

Anisotropy factor

Deoxyhaemoglobin

Oxyhaemoglobin

Hypoxic-Ischaemic Encephalopathy

Inverse Least Squares

Interstitial Fluid

Infrared Tympanic Thermometry

Complex refractive index

Absorption coefficient

Monte Carlo

Multiple Linear Regression

Magnetic Resonance Imaging

Magnetic Resonance Spectroscopy

Scattering coefficient

Transport scattering coefficient 
$\mathrm{NADH}$

NIR

NIRS

NMR

OD

PC

PCA

PCR

PMDF

QE

QTH

RMS

RTE

SDS

SEC

SEP

$\mathrm{S}_{\mathrm{mc}} \mathrm{O}_{2}$

$\mathrm{S}_{\mathrm{mt}} \mathrm{O}_{2}$

SNR

TM

WDPL
Refractive index

Nicotinamide Adenine Dinucleotide

Near-Infrared

Near-Infrared Spectroscopy

Nuclear Magnetic Resonance

Optical Density

Principal Component

Principal Component Analysis

Principal Component Regression

Photon Measurement Density Function

Quantum Efficiency

Quartz Tungsten Halogen

Root Mean Square

Radiative Transfer Equation

Second Derivative Spectroscopy

Standard Error of Calibration

Standard Error of Prediction

Mean cerebral oxygen saturation

Mean tissue oxygen saturation

Signal-to-Noise Ratio

Tympanic Membrane

Wavelength Dependence of Pathlength 


\section{Chapter 1}

\section{Introduction}

This chapter introduces the objective of this project: to non-invasively monitor temperature in the human body using near-infrared spectroscopy.

In Section 1.1 a synopsis of the project is given, establishing the clinical need for non-invasive tissue temperature monitoring and the proposed implementation of this using near-infrared spectroscopy. Section 1.2 discusses the medical background pertinent to the primary application of this method, the measurement of cerebral tissue temperature in new-born infants during hypothermic treatment. In Section 1.3 the technique of near-infrared spectroscopy is introduced, discussing its current application to physiological monitoring, with emphasis on new-born infants, and its suitability as a technique for tissue temperature monitoring. Finally, Section 1.4 summarises the details of the subsequent chapters in this thesis, highlighting the development of the project towards its goal. 


\subsection{Objective and Motivation}

Measurement of temperature within the body is an important indicator of metabolic and thermoregulatory activity (Togawa, 1985). Whilst core body temperature has traditionally been measured at rectal, sublingual and axillary sites, certain clinical studies require the measurement of temperature within a specific region of the body. For example, local temperature monitoring during studies of skeletal muscle activation can provide information about the temperature-dependence of oxygen consumption by muscle tissue (Ferretti et al, 1995). Measurement of tissue temperature is also important during clinical therapies such as hyperthermia, in which the local tissue temperature is raised above $40^{\circ} \mathrm{C}$ to eradicate tumour cells (Carter et al, 1998). In contrast, hypothermia has been induced in patients undergoing major surgical operations, e.g. cardiopulmonary bypass, to protect the brain against oxygen deprivation (Ginsberg et al, 1992). The risk of permanent brain damage in new-born infants suffering from birth asphyxia has led to recent interest in the use of mild hypothermia as a neuroprotective therapy. To determine its efficacy, brain temperature must be monitored non-invasively and continuously throughout the treatment. Conventional methods of determining brain temperature, however, have proved to be inaccurate during hypothermia (Haaland et al, 1996). The need for a non-invasive technique of monitoring cerebral temperature has therefore arisen.

The technique of near-infrared spectroscopy (NIRS) has become well-established as a non-invasive method of monitoring cerebral oxygenation in new-born infants. The relatively low absorption of near-infrared (NIR) light in biological tissue allows the light to penetrate the skin and skull layers to sample the brain tissue beneath. The neonatal brain is comprised predominantly of water. The temperature-dependence of the water absorption spectrum in the mid-infrared, where the fundamental molecular vibrations occur, has been extensively documented. Although absorption by water in the NIR is significantly lower, the NIR absorption bands also exhibit a temperature response, which may in theory be able to indicate tissue temperature in the brain during hypothermic treatment.

It is the aim of this project to exploit the temperature-dependence of the water absorption spectrum as a means of determining tissue temperature in the neonatal brain. The challenge is to account for the absorption by other compounds in the brain, such as haemoglobin, and the non-linear effects of light scattering. This technique could potentially be used in conjunction with other NIRS methods for determining blood flow or tissue oxygen saturation during mild cerebral hypothermia. 


\subsection{Clinical Background}

As mentioned in Section 1.1, one of the motivations for this project is the need for a noninvasive technique capable of monitoring cerebral temperature in birth-asphyxiated infants during mild hypothermia. This section describes the clinical aspects of birth asphyxia and the potential of mild hypothermia to prevent long-term cerebral damage.

\subsubsection{Birth Asphyxia}

The term birth asphyxia, meaning 'without pulse', is used to describe an injury sustained by the new-born infant around the time of birth which results in hypoxia, or poor tissue oxygenation. The injury usually occurs due to abnormal exchange between the placenta and the foetus during labour. If the umbilical cord is compressed, for example, hypoxia may be accompanied and further exacerbated by ischaemia, a reduced blood flow to some or all of the baby's organs. The result is known as a hypoxic-ischaemic injury, or 'insult'.

Birth asphyxia affects as many as six in every one thousand term births (Robertson and Edwards, 1998), with varying degrees of severity. Approximately $50 \%$ of asphyxiated term infants recover completely on resuscitation and show no abnormal neurodevelopment. However, significant cases of asphyxia result in damage to the brain known as hypoxicischaemic encephalopathy (HIE), which can lead to death in the early neonatal period. Of those who survive HIE approximately $25 \%$ will suffer from permanent neurological

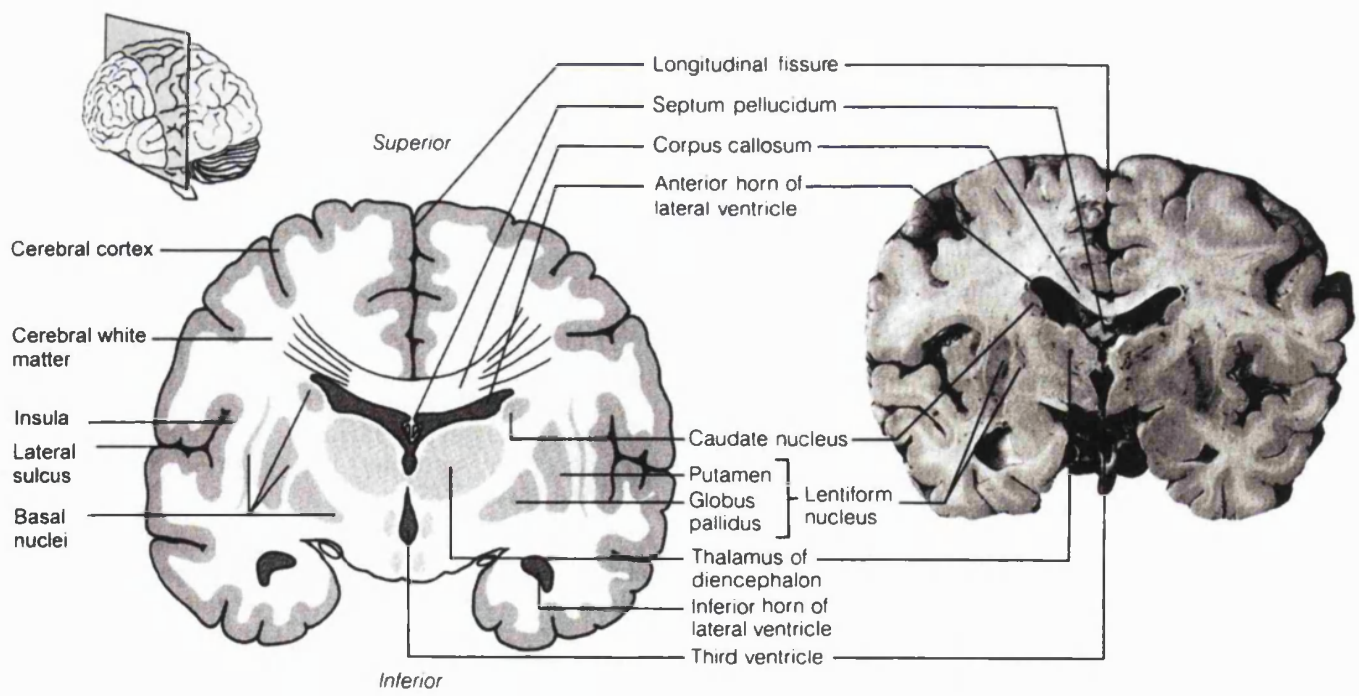

Figure 1.1: Cross-sectional view of the (adult) cerebral hemispheres (Marieb 1995) indicating the areas affected by HIE in new-born infants: the cortex, the sub-cortex and the basal nuclei (see text for details). 
disorders (Vannucci and Perlman, 1997). The majority of damage caused by HIE in term infants is located in the cortex (grey matter), subcortex (white matter) and the basal nuclei (Campistol et al, 1999). Figure 1.1 shows a cross-sectional representation of the (adult) cerebral hemispheres in which these areas are identified (Marieb, 1995). The cortex is responsible for controlling motor activity and interpreting sensory inputs. It is also involved in the intellectual and emotional processing of conscious thought and behaviour. The nerve axons in the sub-cortical white matter provide the route by which information is passed between different parts of the cerebral cortex and also to other parts of the central nervous system (CNS). Deep within the hemispheres are the basal nuclei, or basal ganglia, clusters of nuclei that, in tandem with the motor cortex, are involved in control of movement. Cerebral palsy (CP) is a neurological disorder caused by a lack of development or damage to the brain, characterised by impaired muscle control or paralysis. The most common form, known as spastic CP, occurs as a result of damage to the cerebral cortex. HIE is estimated to be the cause of $20 \%$ of all CP cases (Patel and Edwards, 1997).

A complication in the clinical assessment of HIE is the possibility that certain symptoms may not be apparent for some time after the initial hypoxic-ischaemic insult. Phosphorus magnetic resonance spectroscopy ( ${ }^{31} \mathrm{P}$ MRS) has shown that after resuscitation, the ratio of phosphocreatine to inorganic phosphate, $\mathrm{PCr} / \mathrm{P}_{\mathrm{i}}$, in the brain of birth asphyxiated infants is usually normal (Wyatt et al, 1989). In some infants, however, the observed $\mathrm{PCr} / \mathrm{P}_{\mathrm{i}}$ ratio decreases significantly some 12 to 24 hours later, indicating impaired cerebral energy metabolism and the onset of further brain damage (Azzopardi et al, 1989). The mechanism for this 'delayed energy failure', discussed briefly in Section 1.2.2, is not fully understood, but it is believed that the period immediately after resuscitation of the infant provides an interval during which any clinical or pharmalogical intervention may be most efficient in reducing the ultimate severity of the damage caused by HIE.

\subsubsection{Delayed Energy Failure}

In order to produce adenosine triphosphate (ATP), the molecule that stores chemical energy used for all cellular activity, the human brain requires a constant supply of both glucose and oxygen. ATP production is complicated and involves many metabolic pathways, one of these being a process known as oxidative phosphorylation (see Section 2.1.4). Energy released during the process is used to form the high energy bonds of ATP, which is then stored in equilibrium with PCr. Both ATP and PCr are used by the brain for synthetic and ion transport purposes, e.g. in the transmission of neuronal impulses. 
Lorek et al (1994) studied $\mathrm{PCr} / \mathrm{P}_{\mathrm{i}}$ ratios in the new-born piglet during and after an induced hypoxic-ischaemic insult. During a temporary occlusion of the carotid arteries they observed an initial cerebral energy failure. Upon reperfusion the $\mathrm{PCr} / \mathrm{P}_{\mathrm{i}}$ ratio was seen to return to normal. Within several hours, however, the piglets suffered what is commonly termed delayed or secondary energy failure, as indicated by a further decrease in high energy phosphate ratios. During delayed energy failure the brain suffers neuronal death, the extent of which appears to be related to the depletion in high energy phosphates during the initial insult (Mehmet et al, 1994). The mechanism by which delayed cell loss occurs is believed to be that of apoptosis, described as a kind of programmed 'cell suicide' (Edwards and Mehmet, 1996). Apoptosis is usually a controlled event that occurs to a certain extent during normal neurodevelopment (Mazarakis et al, 1997). After a severe hypoxic-ischaemic injury, however, apoptosis is somehow triggered and cascades out of control, causing serious and irreversible brain damage. The trigger mechanism is not fully understood, but an increase in extracellular glutamate concentration is thought to be a contributing factor (Fellman and Raivio, 1997). Glutamate levels, which can be detected by proton magnetic resonance spectroscopy $\left({ }^{1} \mathrm{H} \mathrm{MRS}\right)$, are seen to rise during secondary energy failure (Penrice et al, 1997).

Recently there has been a considerable interest in therapies with the potential to interrupt the sequence of events leading to apoptotic neuronal death in birth asphyxiated infants (Vannucci and Perlman, 1997; Robertson and Edwards, 1998). The 'window of opportunity', during which therapeutic measures are most likely to protect the brain from further damage, has been identified as the interval following resuscitation of the infant. The following section will discuss the induction of clinical hypothermia as a neuroprotective therapy, and its implications in both animal models and human infants.

\subsubsection{Clinical Hypothermia}

Clinical hypothermia has long been known to protect the brain from permanent damage during a hypoxic-ischaemic insult. Reports of children surviving near-drowning experiences in cold waters have shown that complete neurological recovery is possible, even after submersion periods of up to one hour (Siebke et al, 1975; Sekar et al, 1980; Bolte et al, 1988). The technique of 'deep' clinical hypothermia, a lowering of core temperature to $15-25^{\circ} \mathrm{C}$, has for many years been used to protect the brains of infants suffering from congenital heart disorders during cardiac arrest and cardiopulmonary bypass in open-heart surgery (Bigelow et al, 1950; Dickinson and Sambrooks, 1979; Greeley et al, 1989). 
Hypothermia is thought to prevent or minimise brain damage caused by hypoxicischaemia by reducing the metabolic demand for oxygen, thus maintaining normal cerebral levels of high energy phosphates (Ginsberg et al, 1992; Williams et al, 1997), although other mechanisms are also believed to contribute (Colbourne et al, 1997). Prolonged deep hypothermia, however, can have adverse physiological effects (Edwards et al, 1998), hence researchers have turned towards investigating the efficacy of 'mild' hypothermia, i.e. a reduction in temperature of about 3 to $6^{\circ} \mathrm{C}$. Although dependent on the length of the applied therapy, mild hypothermia appears to be without the systemic side effects associated with deep hypothermia such as respiratory disorders (Thoresen, 1999; Simbruner et al, 1999). However, since intra-ischaemic hypothermia is only applicable in cases of 'pre-elective' care, e.g. heart bypass operations, there has been recent interest in the neuroprotective capabilites of post-ischaemic mild hypothermia.

The findings of recent studies of post-ischaemic hypothermia in animal models are varied, though promising. In summary, it appears that post-ischaemic hypothermia can provide a similar degree of neuroprotection to intra-ischaemic hypothermia (Huh et al, 2000 ), and that the extent of the delayed energy failure is significantly reduced compared to normothermic models (Thoresen et al, 1995). Moreover, in long-term studies of neurodevelopment, outcome has been related to the duration of the post-ischaemic cooling (Colbourne and Corbett, 1994) and the time delay between the insult and the induction of hypothermia (Colbourne and Corbett, 1995). Although these findings show that mild hypothermia has a secure place in the treatment of hypoxic-ischaemic injuries, the situations

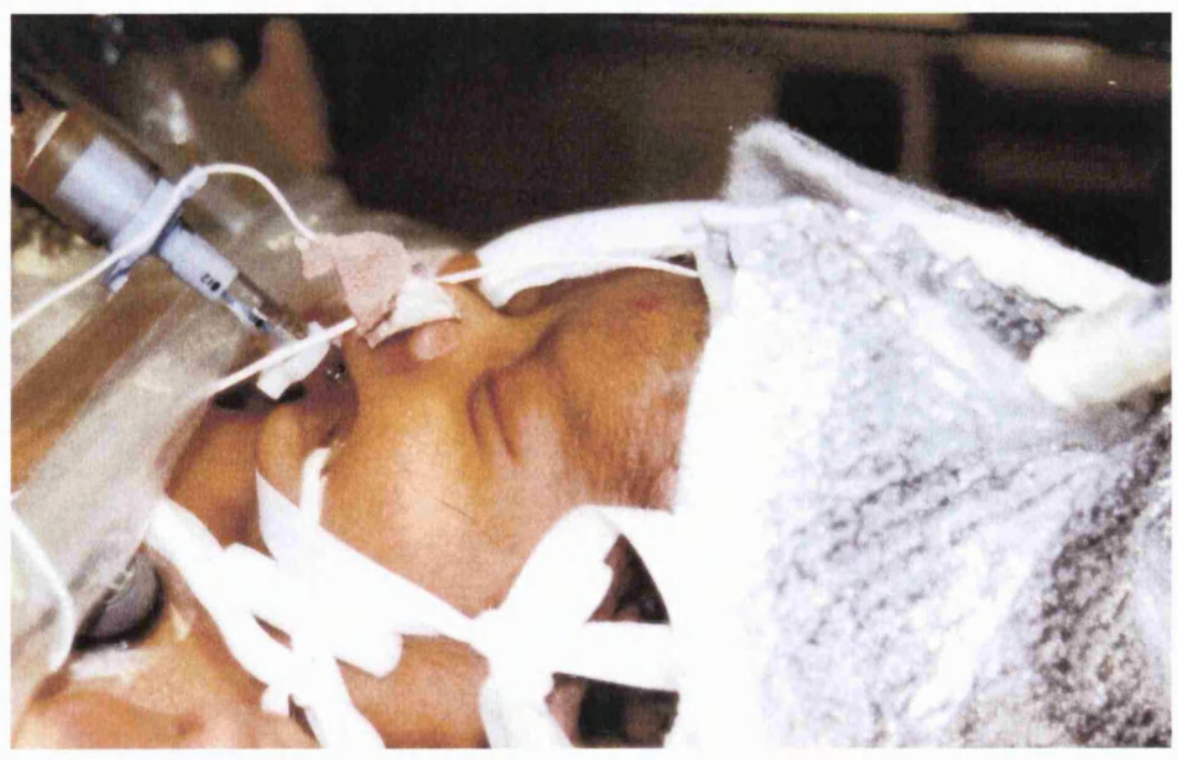

Figure 1.2: Cooling cap applied to a new-born infant for the induction of mild hypothermia by selective head cooling (Wyatt, 1999). 
faced by paediatricians in neonatal intensive care units are somewhat different to those found with animal models in the laboratory. Clinical trials of hypothermic treatment in asphyxiated infants are thus progressing cautiously, and much deliberation has gone into establishing a suitable protocol (Edwards and Azzopardi, 1998). For example, regarding the question of whether whole body or selective head cooling should be used to induce hypothermia in the brain, Edwards et al (1998) point out that the need for prolonged mild hypothermia must be balanced by the need to minimise any associated side effects. In a preliminary study by Gunn et al (1998) a group of term infants with moderate to severe HIE were cooled using selective head cooling for up to 72 hours, in which cold water is circulated around a 'cooling cap' placed on the head whilst the body is warmed by overhead heaters, as shown in Figure 1.2 (Wyatt, 1999). A mild systemic hypothermia was achieved, as recorded by continuous rectal and nasopharyngeal temperature monitoring, and no adverse effects due to hypothermia were observed. The authors concluded that mild hypothermia induced by selective head cooling is a safe way of lowering cerebral temperature in term infants suffering from birth asphyxia.

\section{$1.3 \quad$ Near-Infrared Spectroscopy}

In the near-infrared region of the spectrum, 650-1050 nm, absorption in biological tissue is dominated by water and haemoglobin. The absorption by these chromophores is relatively low compared to the visible and mid-infrared regions (see Section 2.1). This enables the light to travel deep into most tissues, its propagation governed by scattering processes, and hence makes it a suitable probe for in vivo measurements.

The first application of NIRS to clinical measurements was in the field of oximetry, i.e. the spectroscopic measurement of haemoglobin oxygen saturation. Oximetry is based on the oxygen-dependence of the haemoglobin absorption spectrum (Section 2.1.2), in which comparisons of haemoglobin absorption at different wavelengths can yield the degree of oxygen saturation in the blood. A concise review of the development of in vivo NIRS oximetry up to the 1970s, when the first commercial oximeter became available, is given by Rolfe (2002). Since the beginning of the 1970s the field of in vivo NIRS has expanded dramatically. In addition to the mean tissue oxygen saturation indicated by in vivo oximetric measurements, NIRS can monitor oxidative processes at the intracellular level via the redox-dependence of the cytochrome $c$ oxidase absorption spectrum. Cytochrome $c$ oxidase is an intracellular respiratory enzyme which interacts directly with oxygen in the 
cells (Section 2.1.4), hence changes in its absorption can indicate the availability of cellular oxygen (Wray et al, 1988).

The development of clinical NIRS in the last 15-20 years has seen an increased focus on the monitoring of cerebral function in the neonate (Cooper et al, 1996; Patel et al, 1998; Wolf et al, 2000; Wyatt et al, 1990). The non-invasive nature of the technique, its portability and relatively low cost make it an ideal choice for studying cerebral haemodynamics at the cot-side of new-born infants (Meek et al, 1999). As suggested in Section 1.1, therefore, the proposed method of monitoring brain temperature by NIRS could be performed simultaneously with already well-established measurements of cerebral function.

\subsection{Thesis Overview}

Having introduced the main objective of the project, this final section will give an overview of the development of the thesis towards the goal of non-invasive tissue temperature measurement by NIRS.

Chapter 2 introduces the concept of light propagation in biological tissue and discusses the physical and chemical processes involved in absorption and scattering of light by different tissue components and in whole tissues. Models of light transport based on radiative transfer theory are described, and their use in determining the optical properties of tissue is established.

In Chapter 3 the technique of NIRS is discussed in greater detail, introducing important definitions relevant to the in vivo monitoring of physiological parameters. The various types of NIRS measurement are described, with emphasis on the steady-state techniques employed in this project.

Chapter 4 discusses the measurement of body temperature by both conventional techniques and those currently under research development. The need for an alternative method of monitoring cerebral tissue temperature is highlighted by studies describing the poor agreement between invasively measured brain temperatures and those obtained using conventional methods. The optical properties of tissue as a function of temperature are reviewed and the feasibility of using NIRS to monitor tissue temperature is once again addressed.

Chapter 5 describes the experiments designed during the course of this project to accurately determine the temperature-dependence of the NIR water absorption spectrum. The potential effects of water binding on this temperature-dependence are also investigated. 
The water spectrum is calibrated against temperature using a variety of multi-variate calibration techniques. The ability of each of these methods to determine temperature in 'unknown' samples is assessed.

Chapter 6 describes the temperature-resolved measurements of ex vivo and in vivo tissues and a tissue-like phantom. The results of these experiments are discussed in terms of the changes in optical properties with temperature described in Chapter 4 .

In Chapter 7 various methods of predicting temperature from the temperature-resolved measurements of Chapter 6 are investigated, including the use of second-derivative spectroscopy, non-linear optimisation and spatially-resolved techniques. The calibration of the water spectrum described in Chapter 5 is used to predict temperature from reconstructions of the tissue absorption spectrum.

Chapter 8 gives an overall review of the techniques developed in this thesis and their ability to predict tissue temperature from NIRS measurements. Suggestions for additional work are also discussed, highlighting the direction from here towards the ultimate aim of the project, to monitor in vivo tissue temperature non-invasively. 


\section{Chapter 2}

\section{Light Transport in Biological Tissue}

In order to discuss the technique of NIR tissue spectroscopy in more detail, it is important to establish an understanding of the manner in which light propagates through biological tissue. This chapter therefore describes the mechanisms that govern light transport through physical media and identifies the components of biological tissue that are responsible for these processes.

Section 2.1 describes the concept of light absorption pertaining to the (near-) IR region of the spectrum, introducing laws and definitions relevant to its quantification and measurement. This section also presents the constituents of biological tissue responsible for the absorption of NIR light, highlighting the important features of their absorption spectra. In Section 2.2 the basic theory behind light scattering and the parameters used to define it are introduced. The discussion is limited to elastic scattering, in which there is no loss in energy of the scattered light, on the grounds that non-linear scattering effects are generally not significant in the NIR region. The biological structures that give rise to scattering in tissue are also identified. Section 2.3 discusses the various models commonly used to describe the transport of light through turbid media, i.e. those in which multiple scattering occurs. In particular the discussion concentrates on the assumptions and approximations made for each model and hence their validity in 'real' situations. 


\subsection{Light Absorption}

When light radiation is incident on matter composed of discrete electrical charges, the charges are forced to oscillate at the frequency of the incident electric field. The range of frequencies covered by radiation in the infrared region of the electromagnetic spectrum $(\sim 300 \mathrm{THz}-300 \mathrm{GHz})$ is comparable to the natural frequencies at which atoms or molecules will vibrate in the absence of an applied field. Thus when infrared radiation is incident on a system of matter, resonance will occur around the natural frequencies, whereby energy is transferred from the incident field to the system and its amplitude of vibration is greatly increased. Although the lifetime of the excited state is around $10^{-7}$ to $10^{-8}$ seconds, the atoms or molecules will usually lose their energy by colliding with one another within $10^{-12}$ seconds, thereby raising the kinetic energy of the other particles involved in the collisions. Hence, the energy associated with the incident field is most often dissipated as heat within the medium. This process is known as absorption. The overall effect of absorption is a reduction in the intensity of the light beam traversing the medium. For a more detailed discussion on the subject of absorption of electromagnetic radiation the reader is referred to Bohren and Huffman (1983), or Jenkins and White (1981).

A relationship between the absorption of light in a purely absorbing medium and the thickness of the medium was first determined in 1729 by Bouguer (1729). Some years later Lambert (1760) derived the following mathematical expression for the relationship, known as the Lambert-Bouguer law

$$
\frac{\mathrm{d} I}{I}=\mu_{a} \mathrm{~d} l
$$

which describes how each successive layer $\mathrm{d} l$ of the medium absorbs the same fraction $\mathrm{d} I / I$ of the incident intensity $I$ for a constant $\mu_{a}$, the latter known as the absorption coefficient with units of inverse length (usually $\mathrm{mm}^{-1}$ ). For an incident intensity $I_{0}$, therefore, the transmitted intensity $I$ through a distance $l$ will be

$$
I=I_{0} e^{-\mu_{a} l}
$$

The absorption coefficient $\mu_{a}$ can thus be interpreted as the probability that a photon will be absorbed by the medium per unit length. The reciprocal of the absorption coefficient, known as the absorption length, is the distance required for the intensity of the beam to 
fall to $\mathrm{e}^{-1}$ of the initial intensity. When Equation (2.2) is expressed in base 10 logarithms

$$
I=I_{0} 10^{-K l}
$$

then the constant $K$ is known as the extinction coefficient. The absorbance of the medium is defined as the $\log _{10}$ ratio of the incident and transmitted intensities

$$
\begin{aligned}
A & =\log _{10}\left(\frac{I_{0}}{I}\right) \\
& =K l
\end{aligned}
$$

where the unit of absorbance is the 'optical density' (OD). Hence, the units of $K$ are OD per unit length (usually $\mathrm{OD} \mathrm{cm}^{-1}$ ). The extinction coefficient and the absorption coefficient are conceptually the same, differing only by the base of the logarithm used in the Lambert-Bouguer expression. For the same unit length, therefore, the extinction coefficient is related quantitatively to the absorption coefficient by a factor of 0.434 .

In 1852 Beer determined that the absorption coefficient of a compound is linearly related to its concentration $c$ diluted in a non-absorbing medium (Beer, 1852)

$$
\mu_{a}=\alpha c
$$

where $\alpha$ is known as the specific absorption coefficient. Substituting for $\mu_{a}$ in the LambertBouguer law gives what is known as the Beer-Lambert law

$$
I=I_{0} e^{-\alpha c l}
$$

Expressing the Beer-Lambert law in $\log _{10}$ gives

$$
I=I_{0} 10^{-\varepsilon c l}
$$

where $\varepsilon$ is the specific extinction coefficient, usually expressed in units of OD cm${ }^{-1} \mathrm{mM}^{-1}$. In a solution containing a mixture of $n$ absorbing compounds, the total absorbance is the sum of the individual extinction coefficients multiplied by the distance $l$

$$
\begin{aligned}
A & =\left(K_{1}+K_{2}+\cdots+K_{n}\right) l \\
& =\left(\varepsilon_{1} c_{1}+\varepsilon_{2} c_{2}+\cdots+\varepsilon_{n} c_{n}\right) l
\end{aligned}
$$


The Beer-Lambert law is only valid under certain limited conditions: the light entering the medium must be monochromatic and perfectly collimated, and the medium itself must be purely and uniformly absorbing. Therefore certain errors will arise when applying the law to practical spectroscopic measurements since, for example, even lasers are not perfectly monochromatic. The consequences on experimental measurements of the limitations imposed by the Beer-Lambert law have been discussed elsewhere (Cope, 1991).

There are many compounds in biological tissue which absorb light radiation, collectively known as tissue chromophores, each of which has its own unique spectrum. As expressed in Equation (2.8), the total extinction coefficient of a mixture of compounds is equal to the sum of their individual extinction coefficients, weighted by their relative concentrations. Therefore, approximating tissue as a homogeneous mixture of compounds, the overall light absorption in tissue at a given wavelength depends on the type and concentration of chromophores present. The following sections will discuss the chromophores present in biological tissues and their absorption spectra in the NIR.

Although the familiar term 'absorption' is used throughout this thesis, values for the absorption coefficients are given in units of $\mathrm{OD} \mathrm{cm}^{-1}$, since the spectroscopic measurements made during this project are expressed in terms of OD ( $\log$ base 10). In actual fact, $\mathrm{OD} \mathrm{cm}{ }^{-1}$ is the unit of the extinction coefficient $K$. However, the term 'absorption' and its symbol $\mu_{a}$ are used in preference to 'extinction' (and $K$ ), since the latter has less obvious meaning and in some texts is associated with attenuation due to both absorption and scattering. For consistency the scattering coefficient is also converted to $\mathrm{OD} \mathrm{cm}^{-1}$, although it is more normally expressed in natural logs, as it is originally defined.

\subsubsection{Water}

Water is the most abundant chemical substance in the human body, accounting for 60 to $80 \%$ of total body mass (Marieb, 1995). The water content varies with tissue type and is age and gender-dependent. For example, the new-born brain comprises $90 \%$ water by mass, whereas the water content in adult skeletal muscle is around $74 \%$ (White et al, 1991). Because of its high concentration in most biological tissue, water is considered to be one of the most important chromophores in tissue spectroscopy measurements. The absorption spectrum of water is shown in Figure 2.1 over the wavelength range 200-10,000 $\mathrm{nm}$ (Hale and Querry, 1973) and on an expanded scale from 650 to $1050 \mathrm{~nm}$ (Cooper et al, 1996). Between 200 and $900 \mathrm{~nm}$ there exists a region of relatively low absorption. Above $900 \mathrm{~nm}$ the absorption coefficient increases fairly rapidly to a peak at about $970 \mathrm{~nm}$, 

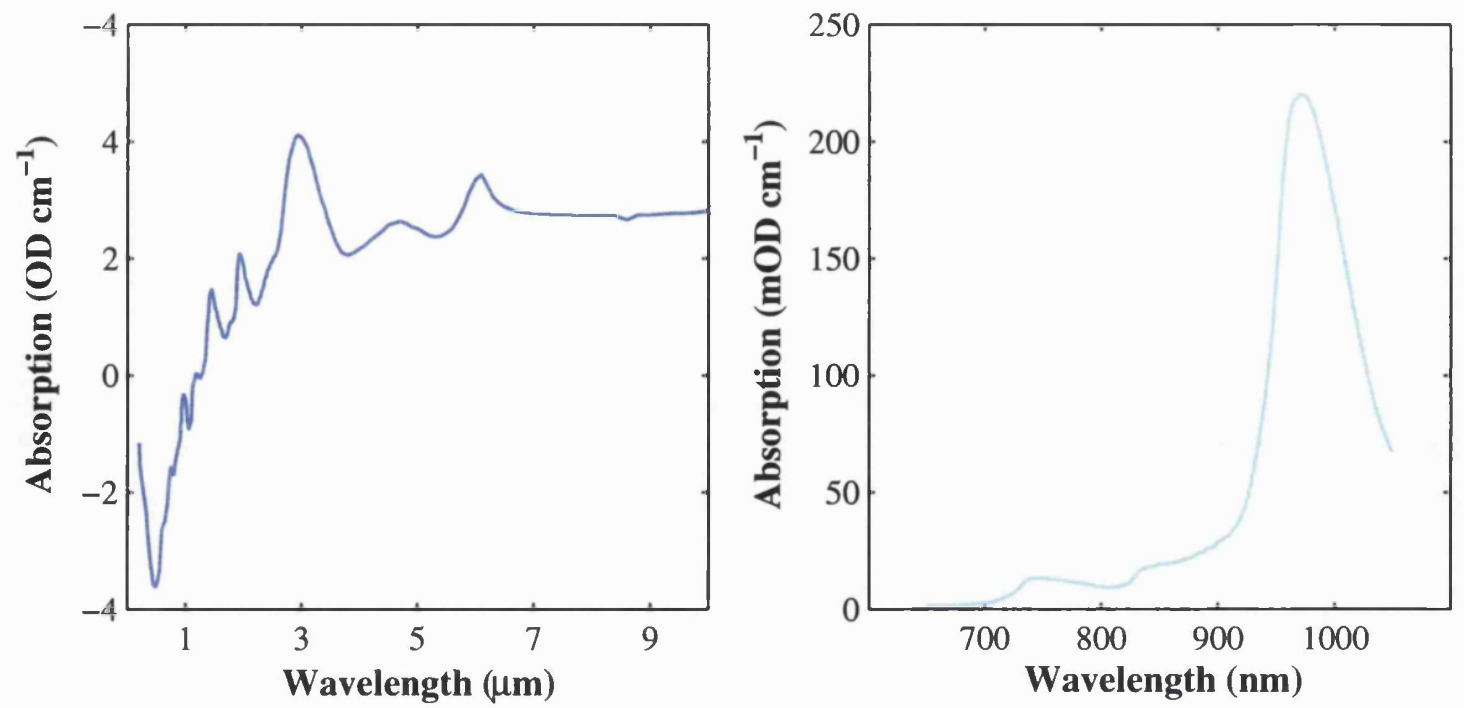

Figure 2.1: The absorption spectrum of pure water. Left: plotted on a $\log _{10}$ scale from 200-10,000 nm (Hale and Querry, 1973), note the use of $\mu \mathrm{m}$ for clearer display. Right: the NIR region from $650-1050 \mathrm{~nm}$ (Cooper et al, 1996), note the use of $\mathrm{mOD} \mathrm{cm}^{-1}$.

and following a minor trough continues to increase at longer wavelengths into the midinfrared. The region of low absorption acts as a 'window' of transparency, allowing NIR spectroscopic measurements through several centimeters of tissue to be made.

Much of the water in the body is not in the 'pure' form for which the above water absorption spectrum was measured but instead is hydrogen-bonded to biomolecules and ions. In nuclear magnetic resonance (NMR) studies of tissue, different proton relaxation times indicate the varying degrees to which water is bound to macromolecules (MathurDe Vré, 1984). Estimations for the amount of bound water in whole skin, for example, are as much as $90 \%$ of the total skin water content (Gniadecka et al, 1998). Tissue water can be categorised into three main types (Marcus, 1995):

i) strongly-bound water, prevalent at bio-surfaces, e.g. at the phospholipid bilayer surfaces of cell membranes

ii) weakly-bound water, hydrogen-bonded to the strongly-bound molecules

iii) free water, hydrogen-bonded to itself only and with similar properties to bulk or pure water, although the transition from bound to free water is not well-defined (Marcus, 1995; Jendrasiak, 1996).

Due to the effect of hydrogen-bonding strength on molecular vibrations (Eisenberg and Kauzmann, 1969), it is reasonable to expect that bound water will differ spectroscopically from free water (see Section 4.3.1.1 for more on hydrogen-bonding in water). In their 
study of the hydration of globular proteins, (i.e. the binding of water molecules to the proteins), Buontempo et al (1972) observed an absorption band in the mid-infrared, other than that of the $\mathrm{O}-\mathrm{H}$ stretching band of pure liquid water usually observed in that region (Section 4.3.1.1). The observed band was similar to that of strongly hydrogen-bonded ice, hence they attributed it to water molecules that were strongly-bound to the proteins. Schneider et al (1979) measured the mid-infrared spectrum of red blood cell membranes as a function of hydration. As hydration was decreased they observed three difference bands, significantly altered from the pure water band, which they postulated were due to water molecules varying in hydrogen-bonding strength and for which the relative concentrations altered with hydration. Bonner et al (1977) studied the effect of organic solutes on the structure of water via their effect on the NIR absorption band at $1.15 \mu \mathrm{m}$, which they decomposed into three Gaussian components corresponding to water molecules that were doubly-bonded to the same organic molecule, singly-bonded to one organic molecule and doubly-bonded to two different molecules, respectively. The relative intensities of the three components varied between solutes, depending on whether the solute-water interactions were more favourable than the water-water interactions.

NIRS studies of bound water in living tissue are scarce. In a study of the effect of different moisturising products, Martin and Curtis (1993) performed in vivo reflectance measurements on human skin in the region 1200-2400 nm. They assigned the observed bands to weakly-bound water, bulk water and two protein-bound water species. With decreasing humidity the peak positions of the weakly-bound and protein-bound bands were seen to shift to longer wavelengths, with a concomitant decrease in the amplitudes of the bands. The position of the bulk water band did not shift with decreasing humidity, although its intensity was seen to increase. The authors concluded that the shift in positions of the bound water bands was due to stronger hydrogen bonding with decreasing humidity. Moreover, they suggested that the change in band intensities was due to a decrease in the thickness of the stratum corneum, the outermost layer of the skin, with decreasing humidity, thus increasing the relative concentration of bulk water, and decreasing that of bound water, within the volume probed.

In general, it is likely that many factors will affect the absorption band positions and intensities of water in biological tissues. For example, white matter in the brain contains a significantly higher proportion of lipids than grey matter (White et al, 1991), including cholesterol, which is known to form stronger bonds with water than the phospholipids common to most cell membranes (Jendrasiak, 1996). Therefore, grey and white matter may 
contain different concentrations of bulk and bound water, which means the contribution to absorption by water could differ spectroscopically between the two tissues.

\subsubsection{Haemoglobin}

Within the water window of transparency the most dominant absorption of NIR light is by haemoglobin in its various forms. Haemoglobin is carried in red blood cells, or erythrocytes, and constitutes approximately $40-45 \%$ of whole blood. It is responsible for delivering oxygen from the lungs to the body tissues and returning waste gases, such as carbon dioxide, to the lungs to be exhaled. Haemoglobin consists of the protein globin bound to four haem groups. Each haem group contains an iron atom at the centre of a ring-like structure. An iron atom in the ferrous $\left(\mathrm{Fe}^{2+}\right)$ form will bind physically to an oxygen molecule to become oxygenated, as opposed to oxidised which would involve a chemical bond. Thus, one haemoglobin molecule with its four iron centres can carry a total of four molecules of oxygen, in which case it is said to be $100 \%$ saturated. In the oxygenated state haemoglobin is known as oxyhaemoglobin $\left(\mathrm{HbO}_{2}\right)$. The de-oxygenated form, with no oxygen molecules attached, is known as deoxyhaemoglobin (Hb).

The specific absorption spectra of oxy- and deoxyhaemoglobin, shown in Figure 2.2 (Cope, 1991), differ significantly, particularly in the red region of the visible and the NIR. This difference in absorption explains the visible colour difference between venous and arterial blood. Arterial blood, which in adults is usually about $98 \%$ oxygen saturated, is

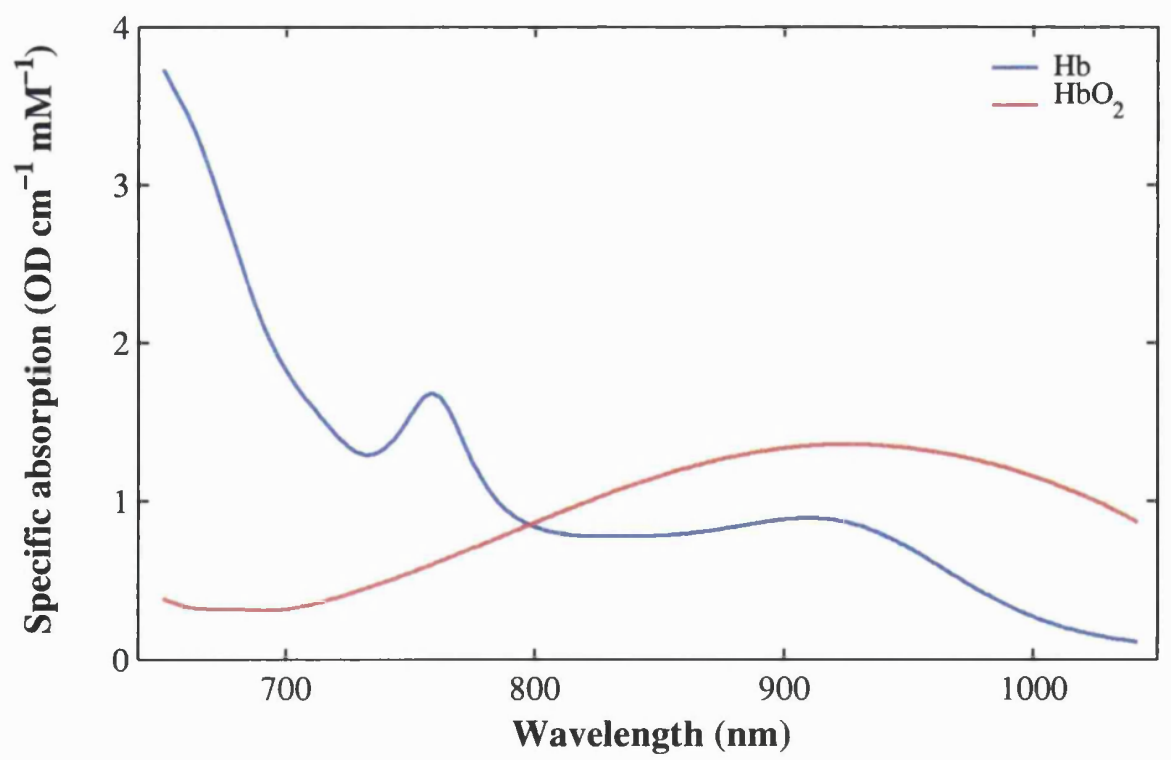

Figure 2.2: Specific absorption spectra of $\mathrm{Hb}$ and $\mathrm{HbO}_{2}$ in the NIR from $650-1050 \mathrm{~nm}$ (Cope, 1991). 
bright red, whereas venous blood, which is approximately $75 \%$ saturated, appears dark red to purple in colour. Although the NIR absorbances are reduced in amplitude relative to those in the visible, the spectra are still sufficiently different to distinguish between the two forms of haemoglobin. A typical value for haemoglobin concentration in the adult brain has been calculated to be $84 \mu \mathrm{M}$ (Cope, 1991). Foetal haemoglobin (HbF) differs from adult haemoglobin (HbA) in that it has a higher affinity for oxygen. This facilitates the uptake of oxygen by the foetus from the placenta, where the diffusion distance is greater than in the lung, and ensures a constant supply of oxygen to the growing cells of the foetus. $\mathrm{HbF}$ is gradually replaced by $\mathrm{HbA}$ in the months after birth. Although the NIR spectra of $\mathrm{HbF}$ and $\mathrm{HbA}$ are slightly different, Wickramasinghe et al (1993) determined no significant difference in the analysis of foetal cerebral oxygenation using either spectra.

Other forms of haemoglobin that absorb in the NIR are carboxyhaemoglobin ( $\mathrm{HbCO}$ ), methaemoglobin (metHb) and sulfhaemoglobin (SHb). However, these derivatives are generally ignored in spectroscopic measurements, either because they have a low percentage concentration in blood or a low specific absorption, or both (Cope, 1991).

\subsubsection{Lipids}

Most of the lipid in the body exists in the form of triglycerides (neutral fats) and is found in subcutaneous tissues and around internal organs. Phospholipids, another group of lipids, are the main component of cell membranes and are thus found in every organ in the body.

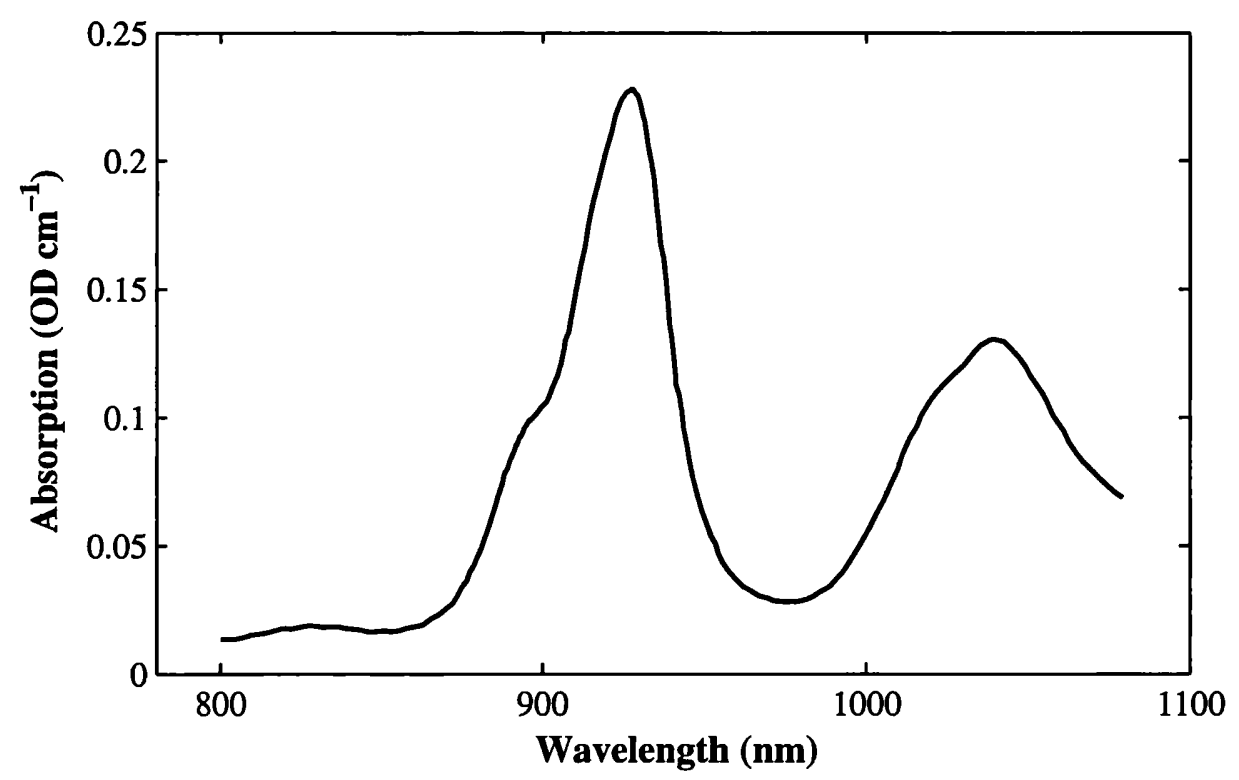

Figure 2.3: The absorption spectrum of pork fat (lipid) in the NIR from $800-1080 \mathrm{~nm}$ (Conway et al, 1984). 
The lipid content in the brain, which also contains steroidal lipids, varies with age from $2.6 \%$ in the new-born to $11.6 \%$ in the adult (White et al, 1991). In adipose tissue, found in the subdermis, the lipid concentration is again age- and gender-dependent, in the range 23-47\% for new-born infants and $68-87 \%$ for adults (White et al, 1991).

The spectrum of pure pork fat between 800 and $1080 \mathrm{~nm}$, shown in Figure 2.3, was measured by Conway et al (1984) and was reported to match the measured spectrum of lipid in muscle tissue. The lipid spectrum is not dissimilar to the water spectrum, but is distinguished from it by the sharp band at about $930 \mathrm{~nm}$ and an absence of one at 970 $\mathrm{nm}$. The importance of lipid as an absorber in NIRS depends upon the tissue in question. Since the water content is much greater than the lipid content in the brain, absorption due to lipid may be insignificant. In the forearm, however, the lipid content is seen to be highly variable, depending on the fat-to-muscle tissue ratio (Matcher et al, 1994), in which case lipid absorption may well be significant in spectroscopic measurements.

\subsubsection{Cytochrome $c$ Oxidase}

Cytochrome $c$ oxidase is the terminal protein in the electron transport chain within the inner mitochondrial membrane (see Section 2.2.2). The electron transport chain is the pathway along which electrons are passed for the purpose of oxidative phosphorylation. This process involves the conversion of adenosine diphosphate (ADP) into adenosine triphosphate (ATP), in which energy for cellular function is stored. The cumulative effect of the electron transport chain is the indirect oxidation of nicotinamide adenine dinucleotide hydride (NADH) by molecular oxygen. The first component of the chain accepts electrons from NADH and passes them on to subsequent components, each of which are at a higher potential than the previous one. The final transfer occurs between the cytochrome $c$ oxidase complex and oxygen, whereby oxygen is reduced to water. The cytochrome $c$ oxidase complex consists of two binuclear units, each containing a haem and a copper centre. The unit involved in the reduction of oxygen contains a haem $a_{3}$ and a $\mathrm{CuB}$ group, whilst the other contains a haem $a$ and a CuA group. Each of the metal groups undergoes oxidation and reduction during the transfer of electrons; the haem groups contain iron, which reversibly oxidises from $\mathrm{Fe}^{2+}$ to $\mathrm{Fe}^{3+}$.

The NIR absorption spectrum of cytochrome $c$ oxidase depends on its redox state, which in turn depends on the availability of oxygen in the cells. When the CuA centre is oxidised a broad absorption band appears, centered at around $830 \mathrm{~nm}$, which does not feature in the reduced spectrum. Thus, it should be possible to make inferences 


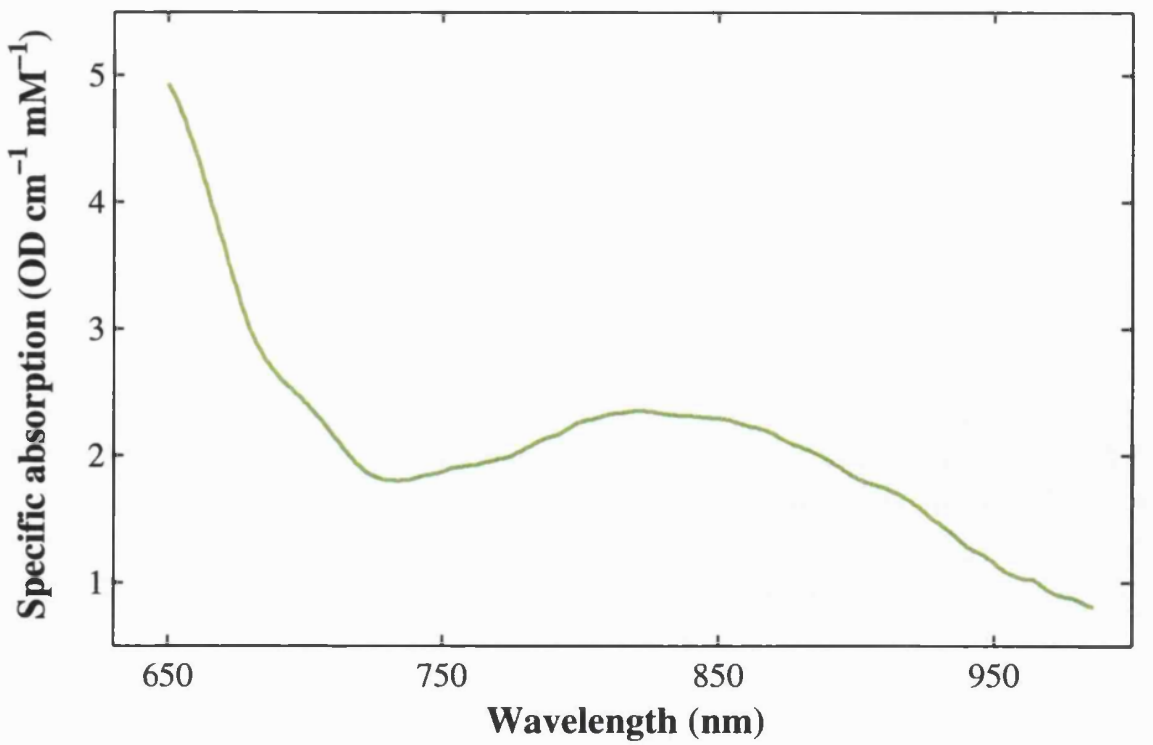

Figure 2.4: Specific difference absorption spectrum of cytochrome $c$ oxidase in the NIR from 650-970 nm (Cope, 1991).

on the availability of oxygen at a cellular level by measuring the difference absorption spectrum between the oxidised and reduced forms of cytochrome $c$ oxidase. This difference spectrum for the in vitro enzyme is shown in Figure 2.4 (Cope, 1991). Many studies have attempted to characterise this spectrum in vivo (Jöbsis, 1977; Wray et al, 1988; Cope, 1991). However, various factors complicate the measurement, in particular the low concentration of cytochrome $c$ oxidase compared to haemoglobin. Concentrations for oxidised cytochrome $c$ oxidase and $\mathrm{Hb}$ of 1.2 and $15 \mu$ Molar respectively have been measured in the neonatal pig brain (Cooper et al, 1999). Although other cytochromes may contribute to the in vivo spectrum, on comparison with in vitro measurements their contributions in the NIR are likely to be small compared to that of cytochrome $c$ oxidase (Cope, 1991).

\subsubsection{Other Chromophores}

There are two other tissue chromophores that are worth mentioning: melanin and myoglobin. Melanin, the pigment found in the epidermal layer of human skin, has a large scattering coefficient in the ultraviolet region, which protects the skin from damaging UV radiation from the sun, and a significant absorption coefficient in the NIR region (Cope, 1991). Thus, although not dependent on tissue oxygenation, the contribution by melanin to the overall light attenuation in in vivo NIRS studies can be considerable. Myoglobin, found within the cells of skeletal muscle, is a red pigment that binds to oxygen, similar to 
haemoglobin in red blood cells and with a similar absorption spectrum in the NIR. However, myoglobin is much less sensitive to tissue oxygenation than haemoglobin and oxygen delivery must be greatly reduced before the myoglobin absorption spectrum is affected (Wang et al, 1990).

\subsection{Light Scattering}

Elastic scattering of light occurs when charged particles in a medium are set into oscillatory motion by the electric field of the incident wave, and re-emit (as opposed to absorb) light of the same frequency as the primary wave. Scattering occurs at non-resonance frequencies, hence the scattered intensities are relatively weak, since the forced vibrational amplitudes of the particles are much smaller than those at natural resonances. In most solids and liquids, however, intermolecular interactions broaden the absorption frequencies such that both scattering and absorption of light occur at all wavelengths.

As a result of scattering, the velocity of light in all matter is less than it is in vacuo. In an optically dense or homogeneous medium, i.e. one in which the molecular separation is much smaller than the wavelength of the incident light, individual atoms or molecules in a medium will scatter the incident radiation in all directions. The phase difference of the scattered light relative to the primary wave will depend on the frequency of the primary wave. In any direction the total scattered field is then a superposition of all the scattered wavelets propagating in that direction. The scattered waves will interfere with the incident wave, modifying its phase and hence the velocity of the light through the medium.

The refractive index of a medium is given by

$$
n=\frac{c}{v}
$$

where $c$ is the speed of light in vacuo and $v$ the speed of light in the medium. The refractive index depends on the number of molecules per unit volume and their polarisability, since the total scattered wave that interferes with the incident wave depends on the amplitudes of, and phase relations between, the individual scattered wavelets. Furthermore, the refractive index for a given medium changes with the frequency of the incident light. This phenomenon is known as dispersion and is due in part to the frequency-dependence of the relative phase change between the incident and scattered waves. Theory has shown, however, that to explain dispersion in real media over a broad spectrum, including the resonance frequencies, the absorption of light must also be considered. Thus, the complex 
refractive index is defined as

$$
N=n+i k
$$

where the real part, $n$, as defined in Equation (2.9), determines the speed of the wave and the imaginary part, $k$, determines the absorption of the wave as it propagates through the medium. From Maxwell's equations $k$ can be related to the absorption coefficient, defined in Section 2.1, as follows

$$
k=\frac{\mu_{a} \lambda}{4 \pi}
$$

where $\lambda$ is the wavelength of the incident light.

\subsubsection{Scattering Theory}

Various theories have been put forward to describe the scattering of light by particles of different shapes and sizes. In general these fall into two categories: single scattering and multiple scattering theories. In single scattering theory it is assumed that the particle separation is sufficiently large, or the number of particles sufficiently small, such that the total scattered wave due to all the particles is small compared to the incident wave (Bohren and Huffman, 1983; van de Hulst, 1981). In other words, the assumption is that the particles do not further interact with the light that has been scattered from neighbouring particles. Single scattering by particles small in diameter compared to the wavelength of the incident light is often known as Rayleigh scattering, after the first scientist to quantitatively study the phenomenon (Rayleigh, 1871a; Rayleigh, 1871b). When unpolarised light is incident on a small particle the intensity distribution of the scattered light is almost isotropic, since the secondary wavelets emitted by the oscillating charges are approximately in phase with one another. At an angle of $90^{\circ}$ to the direction of propagation of the incident light, however, the charges oscillate linearly in one direction only, and thus the light appears to an observer to be linearly polarised. The most important aspect of Rayleigh scattering is its wavelength-dependence, which varies with the inverse fourth power of the wavelength of the illuminating light. Determining the scattered field patterns from particles that are large compared to the wavelength of the illuminating light is an enormously complex task, due to the significant phase differences between the scattered wavelets which must be taken into consideration. However, various approximations can be made to simplify the problem, as is the case in Rayleigh-Gans theory (Rayleigh, 1881; Gans, 1925). In this formulation, the large particle is theoretically split up into many small particles. It is then assumed that the scattered field from each 'small particle' is completely independent of the scattered 
fields from all the others, i.e. is described by Rayleigh scattering. The scattered wavelets from individual particles will interfere with another and the theory must take account of the phase differences, relative to some common origin, between them. Provided the small particles are identical in terms of their size, shape and orientation, it can be shown that all wavelets propagating in the forward direction, i.e. the direction of propagation of the incident beam, are in phase with one another (Bohren and Huffman, 1983). Hence, the amplitudes of the scattered wavelets simply sum to give the total scattered field amplitude in the forward direction. In all other directions constructive and destructive interference will occur. A further analysis known as Mie theory exactly describes both absorption and scattering by a spherical particle of arbitrary radius and refractive index (Mie, 1908). The analysis involves the formal solution of Maxwell's electromagnetic theory for homogeneous spheres using the appropriate boundary conditions (Bohren and Huffman, 1983; van de Hulst, 1981), which in the limit of a small particle reduces to the relatively simple solution for a Rayleigh scatterer. As with the Rayleigh-Gans theory, the scattering is most intense in the forward-direction. However, unlike the Rayleigh-Gans scatterer, the amplitudes of the scattered wavelets in any direction will never be exactly the same, due to the inclusion of absorption effects, therefore complete destructive interference cannot occur.

In the case of single scattering, therefore, a new exponential relationship can be defined for the collimated-beam intensity $I$, relative to the incident intensity $I_{0}$, transmitted a length $l$ through an absorbing medium in which only single scattering occurs

$$
I=I_{0} e^{\mu_{t} l}
$$

where $\mu_{t}$ is the total attenuation coefficient, given by

$$
\mu_{t}=\mu_{a}+\mu_{s}
$$

and $\mu_{s}$ is the scattering coefficient, the probability that a photon will be scattered per unit length. The reciprocal of the total attenuation coefficient, $\mu_{t}^{-1}$, is known as the mean free path, and is the distance travelled by a photon between interactions. In reality, there are many systems for which the assumption that the particles are independent of one another is invalid and multiple scattering becomes important (see Ishimaru (1978) for a comprehensive treatment of multiple scattering theories). Biological tissue illuminated by NIR light is just one example in which multiple scattering cannot be ignored.

Some other useful definitions that describe the parameters involved in light scattering 
should now be introduced. On encountering a scattering particle within a homogeneous medium, photons travelling in a direction $\hat{s}$ are scattered into a new direction $\hat{s}^{\prime}$. The new directions generally do not occur with equal probability and can be described by the differential scattering coefficient, $\mathrm{d} \mu_{s}\left(\hat{s}, \hat{s}^{\prime}\right)$. Integrating over all angles gives $\mu_{s}$, the total scattering coefficient

$$
\mu_{s}=\int_{4 \pi} \mathrm{d} \mu_{s}\left(\hat{s}, \hat{s}^{\prime}\right) \mathrm{d} \hat{s}^{\prime}
$$

Here it is assumed that the scattering coefficient is independent of the original direction $\hat{s}$ of the photons, and that it depends only on the scattering angle between the incident and scattered photons. This may hold true for randomly structured media, but many media have orientation-dependent structure and the scattered intensity distribution will therefore depend on the incident direction. The scattering phase function $p$ is the normalised version of the differential scattering coefficient

$$
p\left(\hat{s}, \hat{s}^{\prime}\right)=\frac{1}{\mu_{s}} \mathrm{~d} \mu_{s}\left(\hat{s}, \hat{s}^{\prime}\right)
$$

such that $\int_{4 \pi} p\left(\hat{s}, \hat{s}^{\prime}\right) \mathrm{d} \hat{s}^{\prime}=1$. The mean cosine $g$ of the scattering angle $\theta$, the angle between the incident $\hat{s}$ and scattered $\hat{s}^{\prime}$ directions, is known as the anisotropy factor

$$
g=\int_{4 \pi} p(\theta) \cos (\theta) \mathrm{d} \hat{s}^{\prime}
$$

If the scattering is completely isotropic then $p$ is equal for all angles and $g$ will be equal to zero. As the particle size increases, however, the intensity distribution increases in the forward direction and $p$ for small angles is much higher than for all other angles. Therefore, the mean cosine tends towards a value of unity, the higher the $g$ value the more forward-peaked the scattering. Combining the scattering coefficient and the anisotropy factor gives the transport scattering coefficient

$$
\mu_{s}^{\prime}=(1-g) \mu_{s}
$$

The significance of the transport scattering coefficient will be discussed in Section 2.3. From the definition of $\mu_{s}^{\prime}$ follows the expression for the transport attenuation coefficient

$$
\mu_{t}^{\prime}=\mu_{a}+\mu_{s}^{\prime}
$$

and its reciprocal, $\mu_{t}^{\prime-1}$, the transport mean free path. Another useful definition is the 
scattering cross-section $\sigma_{s}$, which describes the ability of a particle to scatter light. It is expressed as the effective surface area that a perfectly absorbing disk would have in order to produce the same attenuation of a collimated beam, measured by a collimated detector, as the scattering particle. The scattering coefficient and the scattering cross-section are related as follows

$$
\mu_{s}=\rho \sigma_{s}
$$

where $\rho$ is the particle number density in the medium.

\subsubsection{Light Scattering in Tissue}

Scattering of light in tissue is caused by inhomogeneities such as cell membranes or intracellular structures. The scattering arises due to a relative refractive index mismatch at the boundaries between two such media or structures, e.g. between the extracellular fluid and the cell membrane. Figure 2.5 depicts a 'generalised' cell (Marieb, 1995), i.e. it represents the basic components found in many human cells, but no one cell type in particular. Cells vary greatly in size, from blood platelets of approximately $4 \mu \mathrm{m}$ to nerve cells as much as a metre in length. However, all cells consist of three main components: the cell membrane, the cytoplasm and the nucleus. The cell membrane, which constitutes the outer boundary of the cell, is made up of a phospholipid bilayer, approximately $8 \mathrm{~nm}$

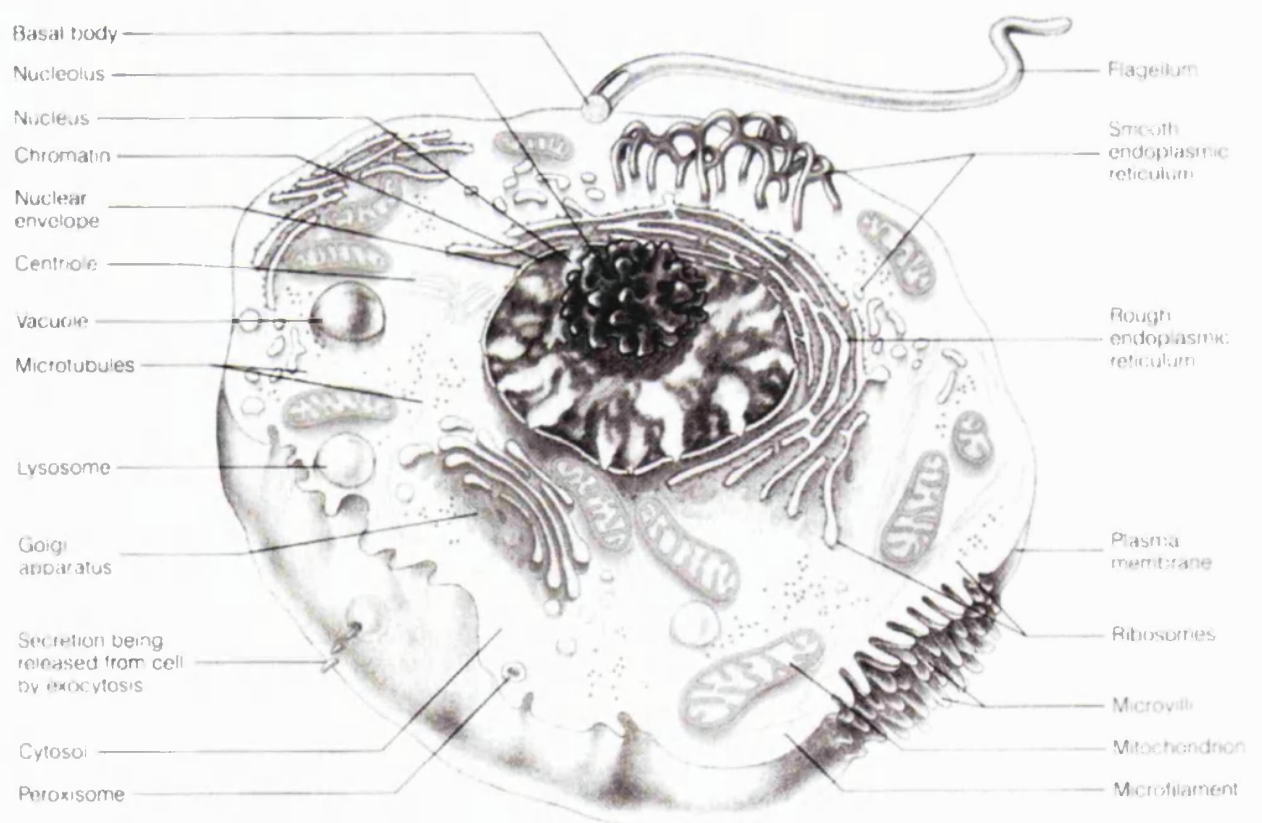

Figure 2.5: A 'generalised' human cell (Marieb, 1995). 
in thickness, with numerous proteins embedded in it. The cytoplasm is the intracellular 'matrix' held within the bounds of the cell membrane. It consists of the cytosol, a water-based fluid within which the other constituents are suspended, the organelles, each of which have a special function in the cell mechanism, and non-functioning units known as inclusions, which include lipid droplets in fat cells and melanin granules in certain skin cells. Cells receives their energy supply from organelles called mitochondria, the number of which reflects a cell's need for energy. The mitochondria, approximately $1-4 \mu \mathrm{m}$ by 0.3-0.5 $\mu \mathrm{m}$ in size (Palade, 1972), are surrounded by a double layer of membrane similar to the outer cell membrane. The nucleus is the largest organelle in the cell and constitutes its control centre. The size of the nucleus varies with cell type, with an average diameter in the order of $5 \mu \mathrm{m}$ (Marieb, 1995). The nucleus, like the mitochondria, is surrounded by a double-layered phospholipid membrane.

In order to understand how light is scattered in tissue it is helpful to study the structures responsible for scattering on a microscopic, i.e. cellular or sub-cellular, level. Each microscopic scattering particle or object will give rise to its own scattering phase function, which depends on the physical properties of the object, as discussed in Section 2.2.1. A single scattering event in tissue can be considered to arise from an 'averaged scattering object,' representing a distribution of scattering objects with an averaged phase function. The contribution of each type of object to the averaged scattering properties in tissue will depend on their individual scattering properties and their relative concentrations.

Mourant et al (1998) studied suspensions of mammalian cells in order to determine the dominant scattering centres in tissue. Comparing their measurements of the transport scattering coefficient $\mu_{s}^{\prime}$ and the anisotropy factor $g$ with predictions from Mie theory, they determined that the observed scattering was due to particles with a distribution of sizes, equivalent to spheres with diameters ranging from about 0.2 to at least $1 \mu \mathrm{m}$. They also measured the phase functions of isolated cell nuclei and mitochondria and compared them to the phase function measured for the cells. The authors concluded that the majority of light scattering from a cell at small angles is due to the nucleus, the smaller organelles such as the mitochondria being responsible for scattering at larger angles. In a more recent study Mourant et al (2000) determined that approximately $55 \%$ of scattering from cells at angles greater than $40^{\circ}$ was due to the internal cellular structures. By comparing scattering from cells and nuclei at different growth phases they concluded that the nucleus and its sub-structures are responsible for a maximum of approximately $40 \%$ of the scattering at any angle and that, as confirmed by the known 
change in mitochondrial content with growth phase, other organelles in the cytoplasm must also contribute significantly to the observed high-angle scatter.

Beauvoit et al (1995) demonstrated a linear increase in $\mu_{s}^{\prime}$ with mitochondrial protein content for various adult rat tissues. The mitochondrial volume fraction was estimated to vary widely in the cells under investigation, from around $8 \%$ in brain to as much as $28 \%$ in liver cells. In one tissue type, white adipose tissue (WAT), $\mu_{s}^{\prime}$ was very high and did not exhibit the same linear dependence on mitochondrial protein content as the other tissue types. The authors commented that the high volume fraction of lipid particles in WAT (approximately $88 \%$ ) and the relatively high refractive index of lipid particles (approximately 1.49 for pure trigylceride (Ross, 1967)) could be responsible for the observed anomaly. In a similar study of scattering from adult rat liver, Beauvoit and Chance (1998) estimated that the mitochondria were responsible for approximately $73 \%$ of total scattering observed from hepatocyte suspensions. Based on the mitochondrial content in whole liver, they determined that $\mu_{s}^{\prime}$ due to the mitochondria was very close to their measured value of $\mu_{s}^{\prime}$. They therefore suggested that, on the premise that the isolated mitochondria are optically comparable to those in situ in the liver, the mitochondrial compartment must be the dominant scatterer in liver tissue.

Another approach to examining the relationship between light scattering and cellular structure is to study the spatial variation in the refractive index on a microscopic level. Beuthan et al (1996) have studied the refractive index of biological cells using a technique called phase microscopy. The phase shift $\Delta \varphi$ of an incident wave (relative to its wavelength) on passing through a sample is related to the difference in refractive index $\Delta n$ between the sample and the surrounding medium

$$
\triangle \varphi=\frac{2 \pi d \triangle n}{\lambda}
$$

where $\lambda$ is the wavelength of the light and $d$ is the diameter of the sample. Beuthan et al (1996) produced phase images of living and dried cells and mitochondria. They showed that the phase shift produced by the mitochondria was small compared to the mean shift due to the whole cell. On inspection of the cross-sectional phase images, they noted that only the nucleus and the cell membrane appeared visibly different from the bulk of the cell. The cell and the mitochondria were modelled using Rayleigh-Gans theory, for which the differential scattering cross-section and phase function can be determined using the relative refractive index of the particle (see Section 2.2.1). They noted that the contribution of 
a particular particle to the total scattering coefficient observed in bulk tissue could then be calculated from the product of the cross-section and number density of the particle. In the same study, Beuthan et al point out that the high refractive index of lipids in the phospholipid membranes (approximately 1.48) relative to the refractive index of the cytoplasm (approximately 1.38) is likely to be a significant cause of scattering from the cell. Modelling the cell as a coated sphere, however, with a coating thickness of $10 \mathrm{~nm}$ and a relative refractive index of 0.1 , they determined that the scattering cross-section of the outer cell membrane alone is sufficient to describe only $1 \%$ of the typical scattering coefficient in tissue. Moreover, a simple model of the mitochondria, which contain between 20 and $40 \%$ of the total membrane volume of the cell (Alberts et al, 1983), as spheres of mean refractive index equal to 1.4 implies that a number density of greater than 1000 mitochondria per cell is required to explain the total scattering coefficient. This has in fact been seen to be the case in hepatic cells, where the number density of mitochondria is estimated to be around 1740 (Beauvoit and Chance, 1998).

The scattering of light by red blood cells should also be considered here. The red blood cell is distinct from the generalised cell described above since it has no nucleus or other intracellular organelles, making it an extremely efficient carrier of oxygen. Haemoglobin molecules (see Section 2.1.2) comprise approximately $97 \%$ of the cell solids (Ross, 1967). The erythrocyte has a biconcave shape, with a main diameter of approximately $8 \mu \mathrm{m}$ and a cross-sectional thickness of 1-2 $\mu \mathrm{m}$. The cell is bound by a membrane approximately $8 \mathrm{~nm}$ in thickness. Fibrous proteins in the membrane allow the cells enough flexibility to deform their shape, in order to fit through capillaries of smaller diameters than themselves. Since there is a refractive index mismatch between the cell membrane and the surrounding medium, i.e. plasma in whole blood, the red blood cell will scatter light. Ross (1967) found the refractive index of the red blood cell to be $1.4 \pm 0.01$ and that of blood plasma to be 1.34. Scattering by a single red blood cell is not easily modelled, however, since it is too large to employ Rayleigh-Gans theory and presents difficulties to Mie theory because of its irregular shape. In spite of this, various authors have made use of these theories (Chadwick and Chang, 1973; Steinke and Shepherd, 1988) and have compared their results to experimental data. Using Mie theory for an uncoated, spherical particle and typical values for the refractive indices, Steinke and Shepherd (1988) modelled scattering from red blood cells in saline suspensions. They found a reasonable agreement between experimental and theoretical values for the scattering cross-section $\sigma_{s}$, but a rather poor correlation between results for the $g$ values, Mie theory predicting a higher anisotropy 
factor $g$, i.e. more forward-peaked scattering, than that found experimentally. As they decreased the concentration of the saline solution, the agreement between theoretical and experimental values of $g$ improved. The authors noted that this was most likely due to the increased sphericity of the erythrocytes as the salt concentration in the suspending solution decreased and the cells swelled due to osmosis of water across the cell membranes.

This section has discussed the microscopic particles responsible for single scattering from tissue cells and their relative contributions to the average scattering phase functions measured. In bulk tissue it is the average scattering (and absorption) properties that are important in describing light transport. The typical mean refractive index for tissue is in the range 1.39-1.41 (Bolin et al, 1989), an exception being adipose tissue at 1.46. Most tissues have a high $g$ value in the range 0.7-0.97 (Flock et al, 1987), which means the scatter is very forward-peaked. (Typical tissue scattering coefficients will be discussed in Section 3.3). It is important then to consider the relevance of measurements of scatter from isolated cells or organelles to the scatter observed in bulk tissue. In tissue as a whole one must also take into account the intercellular order and structures present other than cells, such as collagen fibres. Moreover, in sufficiently thick samples of biological tissue, i.e. greater than the mean free path $\mu_{t}{ }^{-1}$, which is in the region of 10-100 $\mu \mathrm{m}$ (Flock et al, 1987), multiple scattering of light becomes significant.

\subsection{Models of Light Propagation in Turbid Media}

The previous sections have discussed the physical basis for the absorption and scattering of light radiation, and the components of biological tissue responsible for these. The theories discussed in Section 2.2.1 can describe light propagation by both large and small particles (relative to the wavelength of the illuminating light) assuming a small enough collection of particles, or one in which the particles are far enough apart, such that their individual scattered fields do not significantly perturb one other. In biological tissue, however, the scattering particle density is such that the interaction of scattered waves between neighbouring particles cannot be ignored and multiple scattering is said to occur. This section will discuss models of light propagation that are frequently used to describe absorption and scattering in turbid media such as tissue.

It is possible to describe light transport in a regime where multiple scattering dominates using Maxwell's electromagnetic theory (van de Hulst, 1980). It has been noted, however, that solutions to multiple scattering theory will be highly complex (Wilson and Jacques, 
1990; Patterson et al, 1991a), and therefore not altogether practical for the applications associated with clinical NIRS. A more suitable description is one in which the wavelike behaviour of light is ignored and the transport of individual photons, which can be absorbed or scattered, is considered. This treatment is known as radiative transfer theory and is formalised by the radiative transfer equation (RTE)

$$
\frac{1}{v} \frac{\partial}{\partial t} I(\mathbf{r}, t, \hat{s})+\hat{s} \cdot \nabla L(\mathbf{r}, t, \hat{s})=-\left(\mu_{a}+\mu_{s}\right) I(\mathbf{r}, t, \hat{s})+\mu_{s} \int_{4 \pi} p\left(\hat{s}, \hat{s}^{\prime}\right) \mathrm{d}^{2} \hat{s}^{\prime}+\varepsilon(\mathbf{r}, t, \hat{s})
$$

which describes the behaviour of the energy radiance $L(\mathbf{r}, t, \hat{s})$ in the medium, i.e. the energy at position $\mathbf{r}$ moving in the direction $\hat{s}$ per unit of solid angle, per unit of time $t$ and per unit of area normal to the $\hat{s}$ direction (Contini et al, 1997). The other parameters in the equation are $v$, the velocity of the light in the medium, $\mu_{a}$ and $\mu_{s}$, the absorption and scattering coefficients, $p$, the scattering phase function and $\varepsilon(\mathbf{r}, t, \hat{s})$, the radiation source term. Analytical solutions to the RTE that describe photon migration explicitly can be obtained by introducing further approximations. One of the most popular models used in the field of tissue optics is the diffusion approximation, the theory of which will be discussed in Section 2.3.1. A stochastic approach to modelling light propagation can also be taken, again based on radiative transfer theory, and one such technique, the Monte Carlo (MC) method, will be discussed in Section 2.3.2. The 2-flux and multiple-flux models are also useful approximations of the RTE and have been described elsewhere (Patterson et al, 1991a).

\subsubsection{The Diffusion Approximation}

The RTE can be reduced to a set of coupled differential equations by approximating the angular dependence of the radiance by a spherical harmonic expansion of the energy radiance and source terms, $L(\mathbf{r}, t, \hat{s})$ and $\varepsilon(\mathbf{r}, t, \hat{s})$, in Equation (2.21) (Case and Zweifel, 1967). The diffusion approximation is the first-order approximation of the spherical harmonic expansion, also known as the $\mathrm{P}_{1}$ approximation (Patterson et al, 1991a), which gives a set of differential equations from which the diffusion equation can be derived. The assumptions made in the derivation of the diffusion equation are that the source is an isotropic point source and scattering within the medium is (approximately) isotropic (Schweiger et al, 
1995). The time-dependent diffusion equation is given by (Patterson et al, 1989)

$$
\frac{1}{v} \frac{\partial \Phi(\mathbf{r}, t)}{\partial t}-D \nabla^{2} \Phi(\mathbf{r}, t)+\mu_{a} \Phi(\mathbf{r}, t)=\varepsilon_{0}(\mathbf{r}, t)
$$

where $\Phi$ is the photon density or fluence rate, related to the energy radiance as follows

$$
\Phi(\mathbf{r}, t)=\int_{4 \pi} L(\mathbf{r}, t, \hat{s}) \mathrm{d} \hat{s}
$$

$\varepsilon_{0}$ is the isotropic source term and $D$ is the diffusion coefficient, given by

$$
D=\frac{1}{3\left(\mu_{a}+(1-g) \mu_{s}\right)}
$$

where $g$ is the anisotropy factor defined in Equation (2.16) in Section 2.2.1. The transport scattering coefficient, defined in Equation $(2.17)$ as $(1-g) \mu_{s}$, then takes account of the anisotropy of the scattering in an essentially isotropic model. The principle of similarity (Patterson et al, 1991b) states that two media illuminated by a diffuse source will have similar photon distributions if the following conditions are true

$$
\begin{aligned}
\mu_{a 1} & =\mu_{a 2} \\
\left(1-g_{1}\right) \mu_{s 1} & =\left(1-g_{2}\right) \mu_{s 2}
\end{aligned}
$$

where the subscripts 1,2 refer to the two media. Therefore, the transport scattering coefficient effectively represents the isotropic medium, with $g=0$, that gives an equivalent light distribution to an anisotropic medium of scattering coefficient $\mu_{s}$ and anisotropy factor $g$. Hence, by incorporating $g$ in this way the diffusion approximation can model a 'linear anisotropy' that is suitable for biological media.

There are several situations, however, in which the diffusion approximation is not valid. Due to the highly forward-directed nature of scattering in most tissues (Section 2.2.2), the light close to the boundaries and the illuminating source is far from diffuse and the assumption of isotropy is therefore completely invalid. In general, a collimated beam at the surface can be represented by an isotropic point source at a depth $z_{0}$ below the surface (Arridge et al, 1992; Schweiger et al, 1995), which will accurately describe the fluence rate at greater distances from the source than $z_{0}$. The definition of $z_{0}$ is either $\mu_{s}^{\prime-1}$ or $\mu_{t}^{-1}$, depending on the definition of the source term (Contini et al, 1997; Farrell and Patterson, 1992). A further condition of the diffusion approximation is that $\mu_{s}^{\prime}$ is much greater than $\mu_{a}$. If $\mu_{a}$ is comparable to or larger than $\mu_{s}^{\prime}$ then the light will not deviate 
sufficiently from the forward-direction, even at depth, for it to be considered diffuse. In most tissues $\mu_{s}^{\prime}$ is greater than $\mu_{a}$ by one to two orders of magnitude (see Section 3.3), due to the low absorption by water in the NIR (Section 2.1.1). However, there are regions in the body, for example in the cerebrospinal fluid, where a very low $\mu_{s}^{\prime}$ could invalidate the use of diffusion theory as a model of light propagation in tissue and Monte Carlo models become more appropriate (Firbank et al, 1996). Provided $\mu_{s}^{\prime} \gg \mu_{a}$, therefore, light can be considered to be propagating diffusely through a scattering medium beyond a depth of $z_{0}$ from the source. Henceforth, the fluence rate exhibits an exponential decay with increasing depth into the medium (Patterson et al, 1991b; Wilson and Jacques, 1990). The decay constant is known as the effective attenuation coefficient $\mu_{\text {eff }}$, and is predicted by diffusion theory to be

$$
\mu_{e f f}=\sqrt{3 \mu_{a}\left(\mu_{a}+\mu_{s}^{\prime}\right)}
$$

Since the work of this thesis involves time-independent, spatially-resolved measurements of light intensity, it is appropriate to introduce here some time-independent analytical solutions to the diffusion approximation. The steady-state solution to the diffusion approximation for the fluence rate at a distance $(\rho, z)$ from an isotropic point source at $\left(0, z_{0}\right)$ in an infinite turbid medium is given by (Farrell and Patterson, 1992)

$$
\Phi\left(\rho, z_{0}\right)=\frac{1}{4 \pi D} \frac{\exp \left(-\mu_{e f f} r_{1}\right)}{r_{1}}
$$

where $r_{1}$, the distance from the point source to position $(\rho, z)$, is given by

$$
r_{1}=\sqrt{\left(z-z_{0}\right)^{2}+\rho^{2}}
$$

In a semi-infinite turbid medium, the boundary conditions at the surface of the medium become important. If there is no refractive index mismatch between the scattering and the non-scattering medium there must be no photon current back into the scattering medium at the boundary. This condition can be achieved by setting the fluence rate equal to zero at a certain distance above the surface of the medium, at the so-called 'extrapolated boundary' (Farrell and Patterson, 1992). If a mismatch in refractive indices exists at the media interface, the position of the extrapolated boundary must be adjusted to account for the degree of internal reflection at the physical boundary. In general, it can be shown that the position at which to set the extrapolated boundary, to obtain the correct photon current at the surface, is given by $z_{b}=2 \mathrm{~A} D$ (Farrell and Patterson, 1992), where $\mathrm{A}$ is dependent on the relative refractive indices of the media, and is 1 for a matched boundary. 
Using the method of images, Eason et al (1978) demonstrated that the photon current at a given distance above the physical surface, due to a point source at depth $z_{0}$, can be forced to zero if a virtual source is placed at an additional distance $z_{0}$ above the extrapolated plane, i.e. at a height $z_{b}+z_{0}$ above the surface of the medium. The solution for the semi-infinite medium is then given by

$$
\Phi\left(\rho, z_{0}\right)=\frac{1}{4 \pi D}\left(\frac{\exp \left(-\mu_{e f f} r_{1}\right)}{r_{1}}-\frac{\exp \left(-\mu_{e f f} r_{2}\right)}{r_{2}}\right)
$$

where $r_{1}$ was defined in Equation (2.28) and $r_{2}$, the distance from the mirror image source to position $(\rho, z)$, is given by

$$
r_{2}=\sqrt{\left(z+z_{0}+2 z_{b}\right)^{2}+\rho^{2}}
$$

The spatially-resolved diffuse reflectance, i.e. the fraction of light remitted from the medium, at the surface $(z=0)$ is then given by

$$
R(\rho)=\frac{1}{4 \pi}\left(z_{0}\left(\mu_{e f f}+\frac{1}{r_{1}}\right) \frac{\exp \left(-\mu_{e f f} r_{1}\right)}{r_{1}^{2}}+\left(z_{0}+2 z_{b}\right)\left(\mu_{e f f}+\frac{1}{r_{2}}\right) \frac{\exp \left(-\mu_{e f f} r_{2}\right)}{r_{2}^{2}}\right)
$$

Equation (2.31) is usually known as the extrapolated boundary condition (EBC) solution for spatially-resolved reflectance (Kienle and Patterson, 1997). An additional approximation known as the zero boundary condition (ZBC) solution can be made, in which the fluence rate is set to zero at the physical boundary instead of some extrapolated boundary (Kienle and Patterson, 1997). The reflectance derived by assuming this condition is given by Equation (2.31) with $z_{b}=0$, i.e. $r_{1}=r_{2}=r$, as follows

$$
R(\rho)=\frac{z_{0}}{2 \pi}\left(\mu_{e f f}+\frac{1}{r}\right) \frac{\exp \left(-\mu_{e f f} r\right)}{r^{2}}
$$

As mentioned above, the definition of $z_{0}$ given in the literature varies according to the source term used, but in the regime required for validity of the diffusion approximation, i.e. $\mu_{s}^{\prime} \gg \mu_{a}$, the difference between assuming $z_{0}$ to be $\mu_{s}^{\prime-1}$ or $\mu_{t}^{-1}$ is in any case small.

The solutions in Equations (2.31) and (2.32) can be compared to NIRS intensity measurements in order to determine the optical coefficients, $\mu_{a}$ and $\mu_{s}^{\prime}$, of biological tissues, as will be discussed in later chapters. Tissue homogeneity is usually assumed, but a numerical technique known as the finite-element method can be used in conjunction with diffusion theory to model regions with different optical properties (Schweiger et al, 1993). Section 3.1.3 discusses the theory of modelling heterogeneous tissue layers in more detail. 


\subsubsection{The Monte Carlo Method}

The Monte Carlo (MC) method, as applied to the transport of light radiation, is based on the RTE and involves computer-simulated calculations of photon propagation in scattering media. As with radiative transfer theory, MC simulations treat the photons as particles, ignoring their wave-like nature. Therefore, parameters such as the phase and polarisation of the light are usually discounted. Wang and Jacques (1992) argue that this is justifiable since, due to the multiple scattering of the photons, these properties are quickly randomised. In the simplest of $\mathrm{MC}$ simulations photons are injected into a medium individually and their paths traced until they are either absorbed or permanently scattered out of the region of interest (Patterson et al, 1991a). The rules of photon propagation are expressed as probability distributions (hence the name 'Monte Carlo'), which are based on the geometry and optical properties of the medium.

MC simulations are often applied to the propagation of NIR light in tissue (Flock et al, 1989; Firbank et al, 1996; Hielscher et al, 1996), in which the input parameters are usually the absorption and scattering coefficients and the scattering phase function of the tissue. The advantages over the diffusion approximation are that MC simulations do not require that $\mu_{s}^{\prime} \gg \mu_{a}$, and accurate results can be obtained close to sources and boundaries (de Jode, 1999). In addition, complicated geometries and multi-layered tissues in which the optical properties vary spatially can be modelled easily (Hiraoka et al, 1993). However, although potentially highly accurate, the simulations can be expensive due to the long computational times required to achieve such accuracy. For example, for a $1 \%$ accuracy the paths of 10,000 photons must be detected.

A basic method for MC simulation, described by Prahl (1988) and coded by Wang and Jacques (1992), will be outlined here. The method employs the technique of implicit photon capture, also known as survival weighting (de Jode, 1999). Rather than each photon being scattered until its path is terminated by a single absorption process, the photons are given an initial energy or weighting which is reduced, according to the propagation step size, at each scattering site. This improves the statistical efficiency of the method, since it avoids computing the path of photons that may be transported many steps only to be terminated in a single absorption process (Prahl, 1988). The basic steps of a single photon through this type of MC simulation are as follows (Wang and Jaques, 1992):

1. The photon is incident on the tissue boundary at an angle of $\alpha$ and with a weight of $W=1$ 
2. The photon weight is reduced by an amount equivalent to the reflected component at the boundary, $\Delta W$

3. The new direction of the photon is calculated from the incident angle $\alpha$ and the refractive index at the tissue boundary

4. The photon step size $l$ is calculated as follows:

$$
l=-\frac{\ln (\xi)}{\mu_{t}}
$$

where $\xi$ is a randomly generated number between 0 and 1 and $\mu_{t}$ is the total attenuation coefficient. It can be shown that on average the step size $l$ is equal to the mean free path, $\mu_{t}^{-1}$, as expected (Wang and Jaques, 1992)

5. The photon weight is reduced again, due to absorption, by an amount

$$
\triangle W=W \frac{\mu_{a}}{\mu_{t}}
$$

where $W$ is now the weight at the end of step 2

6. A random number, 0 or 1 , is then generated to determine if the photon will be scattered. If the number generated is 0 then no scatter occurs and the procedure is repeated from step 3 . For a value of 1 the photon is scattered

7. The new direction of the scattered photon is calculated using two angles: the deflection angle $\theta$, which has values between 0 and $\pi$, and the azimuthal angle $\varphi$ with values between 0 and $2 \pi$. The deflection angle is calculated from the normalised, cumulatively integrated scattering phase function using $\xi$. The azimuthal angle is uniformly distributed over the interval $0-2 \pi$ and is therefore calculated by $\varphi=2 \pi \xi$

Steps 3-6 are repeated for the desired number of photons. If the simulation involves a more complex heterogeneous model the process returns to step 1 when a tissue boundary is reached, proceeding in the same manner but using the optical properties for the new region. Section 3.1.3 will discuss the application of MC simuations in the modelling of heterogeneous tissue layers in more detail. 


\section{Chapter 3}

\section{Practical Aspects of Tissue NIRS}

This chapter will discuss the practical aspects of NIRS measurements of biological tissue. Since the NIRS studies described in this thesis are based on steady-state (or continuouswave) measurements, the focus shall be on this type of measurement regime.

Section 3.1 describes the use of NIRS measurements to determine tissue optical coeffcients, and discusses aspects of in vivo monitoring of physiological parameters using NIRS. The techniques described can of course be applied in spectroscopic studies of any media, but are discussed here with emphasis on their application to tissue spectroscopy. Some new definitions of particular relevance to steady-state measurements will be introduced. In Section 3.2 the generic equipment used for the steady-state measurements of Chapters 5 and 6 is described, although specific details of each experiment are given in these later chapters. Section 3.3 gives a summary of the optical coefficients previously measured in whole tissues, both in vivo and ex vivo, and includes a brief description of the anatomical features of the component tissues that govern their optical properties. 


\subsection{Tissue NIRS Measurement Techniques}

In Section 1.3 the application of NIRS to the monitoring of tissue oxygenation, haemodynamics and other such physiological parameters was introduced. Tissue NIRS can be implemented by a variety of measurement techniques, all of which attempt to relate the spectroscopic measurement to the physiological parameter of interest via the optical properties of the tissue. Steady-state, or continuous-wave (CW), techniques are based on measurements of the diffuse transmittance or reflectance, i.e. the fraction of the incident signal intensity that is transmitted through or reflected from the tissue. By comparing the spectroscopic measurements to models of light transport in scattering media (Section 2.3), the optical properties of the tissue can be inferred (Simpson et al, 1998). These techniques usually involve illuminating the tissue with either CW irradiation or pulsed irradiation in which the length of the pulse is long in comparison to the transit time of the photons through the tissue. Steady-state measurements of the localised diffuse reflectance at varying distances from the incident irradiation are known as spatially-resolved measurements, from which the optical coefficients can also be obtained (Doornbos et al, 1999). Time-resolved techniques use extremely fast photodetectors to monitor the 'time-of-flight' of individual photons through the tissue (Schmidt et al, 2000). Again, by comparison with models of light propagation, the optical properties of the tissue can be determined from the distribution of signal intensities with photon flight time (Hillman et al, 2001). In the frequency domain the time-of-flight can be related to measurements of the phase shift and modulation depth of the signal, relative to an incident intensity-modulated signal, on passing through the tissue (Kohl et al, 1997). A comprehensive review of these techniques is given elsewhere (Wilson and Jacques, 1990). Since the work of this thesis involves steady-state measurements of tissue (see Chapter 6), this section will discuss the factors involved in the determination of optical coefficients from CW measurements.

\subsubsection{Light Attenuation in Tissue}

Steady-state measurements of tissue are often expressed in terms of the attenuation $A$, defined as the log (base 10) ratio of the incident to remitted (reflected or transmitted) intensities

$$
A=\log _{10} \frac{I_{0}}{I}
$$

where $I_{0}$ is the incident intensity and $I$ is the intensity reflected from or transmitted through the tissue. Since attenuation is defined in the same way as the absorbance for 
a purely absorbing medium (Section 2.1), it is also by convention given the symbol $A$ and has units of OD. However, whereas the absorbance is a linear function of absorption in a non-scattering medium, the attenuation in a turbid medium such as tissue depends non-linearly on both absorption and scattering. The effect of scattering is to increase the distance travelled by the photons relative to a transparent medium, hence the amount of light absorbed on travelling the fixed distance from source to detector will be greater than in the non-scattering medium. The non-linear relationship between attenuation and absorption can be understood intuitively if one considers that as the absorption increases, the mean distance travelled by the photons between the source and detector will tend to decrease, since the photons travelling the longer distances are absorbed before they reach the detector. Thus, although attenuation increases with absorption, the change in attenuation is smaller at higher values of absorption, due to the concomitant decrease in the average pathlength of the photons. (The optical pathlength will be discussed in greater detail in Section 3.1.2.)

The calculation of tissue attenuation, based on the definition given in Equation (3.1), requires that the incident intensity $I_{0}$ is known. However, measurement of the wavelengthdependence of intensity $I_{0}$, delivered to the tissue surface by an optical fibre, say, will be weighted by factors such as the spectral efficiency of the detector (see Section 3.2) and the transmittance of the detecting fibre. These factors are normally termed the 'spectral response' of the system, and can be accounted for by making a reference intensity measurement in the absence of any other attenuator, e.g. through air. Filters with wavelengthindependent attenuation properties, known as neutral density filters, are often used to attenuate the output of the source to a level appropriate for detection. In fact, any attenuator with a spectrally featureless remittance (diffuse transmittance or reflectance) can be used, e.g. an integrating sphere (see Section 3.2). The reference intensity $I_{\text {ref }}(\lambda)$ measured by the system through a neutral attenuator can be given as

$$
I_{\text {ref }}(\lambda)=I_{0}(\lambda) S(\lambda) R^{m}
$$

where $I_{0}(\lambda)$ is the output intensity from the source, $S(\lambda)$ is the spectral response of the system to a unit neutral attenuator and $R^{m}$ is the wavelength-independent remittance of the attenuator used in the measurement. Similarly, the intensity measured through a sample, $I_{\text {sample }}(\lambda)$, is given by

$$
I_{\text {sample }}(\lambda)=I_{0}(\lambda) S(\lambda) R_{\text {sample }}^{m}(\lambda)
$$


where $R_{\text {sample }}^{m}(\lambda)$ is the remittance of the sample. Both the output intensity of the light source and the system response are generally unknown. However, assuming they do not change between reference and sample measurements, it can be seen that these unknown quantities cancel out in the calculation of attenuation using Equation (3.1) if $I_{\text {ref }}$ and $I_{\text {sample }}$ are substituted for $I_{0}$ and $I$ respectively. The calculation therefore yields the true spectral-dependence of the sample attenuation, with respect to that of the neutral attenuator, albeit scaled by the remittance $R^{m}$ and any other potential scaling factor, such as an alteration in the acquisition time of the measurement in the presence of the sample. In general, therefore, spectroscopic measurements of tissue attenuation are relative ones, although absolute CW measurements are possible (Kienle et al, 1996).

As discussed in Section 2.3, the diffuse reflectance is defined as the fraction of the incident intensity remitted from the medium. Attenuation is therefore given by the (negative) $\log$ of the reflectance

$$
\begin{aligned}
R & =\frac{I}{I_{0}} \\
A & =-\log _{10} R
\end{aligned}
$$

The optical properties of tissue can be determined from attenuation measurements by comparing them with an analytical solution for the diffuse reflectance based on diffusion theory (Farrell and Patterson, 1992), or with stochastic models such as the computergenerated Monte Carlo simulations (Flock et al, 1989), described in Section 2.3. For direct comparison with diffusion theory, within the limits of its validity, the refractive index mismatch at the tissue surface and the numerical aperture of the detection fibre should also be considered (Kienle and Patterson, 1997). Whilst it is possible to model almost any physical situation with Monte Carlo simulations, the computing time increases greatly with complexity of the model (Hiraoka et al, 1993). An alternative to these modelling techniques is to determine a linear approximation of the changes in attenuation as a function of changes in absorption (Delpy et al, 1988). Many in vivo NIRS studies are interested only in changes in tissue chromophore concentrations from a physiological 'baseline' and do not require quantification of the absolute absorption coefficients, e.g. (Wyatt et al, 1990). For small changes in attenuation $\triangle A$, the following approximation can be made

$$
\triangle A=\frac{\partial A}{\partial \mu_{a}} \triangle \mu_{a}+\frac{\partial A}{\partial \mu_{s}^{\prime}} \triangle \mu_{s}^{\prime}
$$

If the assumption can be made that changes in tissue scattering during the period of the 
measurement are negligible, then changes in attenuation are given by

$$
\begin{aligned}
\triangle A & =\frac{\partial A}{\partial \mu_{a}} \Delta \mu_{a} \\
& =\frac{\partial A}{\partial \mu_{a}} \varepsilon \Delta c
\end{aligned}
$$

where $\varepsilon$ is the specific absorption coefficient and $\Delta c$ are the changes in the chromophore concentration. The approximation of Equation (3.6) is represented graphically in Figure 3.1, in which attenuation determined analytically using a diffusion theory solution is plotted against absorption, for a fixed scattering coefficient. The local tangent to the curve of attenuation against absorption, i.e. the partial derivative of $A$ with respect to $\mu_{a}$, represents the mean optical pathlength travelled by the light for a given set of optical coefficients, and is known as the differential pathlength (DP)

$$
\mathrm{DP}=\frac{\partial A}{\partial \mu_{a}}
$$

Using Monte Carlo simulations, Delpy et al (1988) have shown that the DP relates directly to the mean transit time of photons through a scattering medium. The following section discusses the DP in greater detail, since its quantification is important for the accurate determination of chromophore concentration changes using this method.

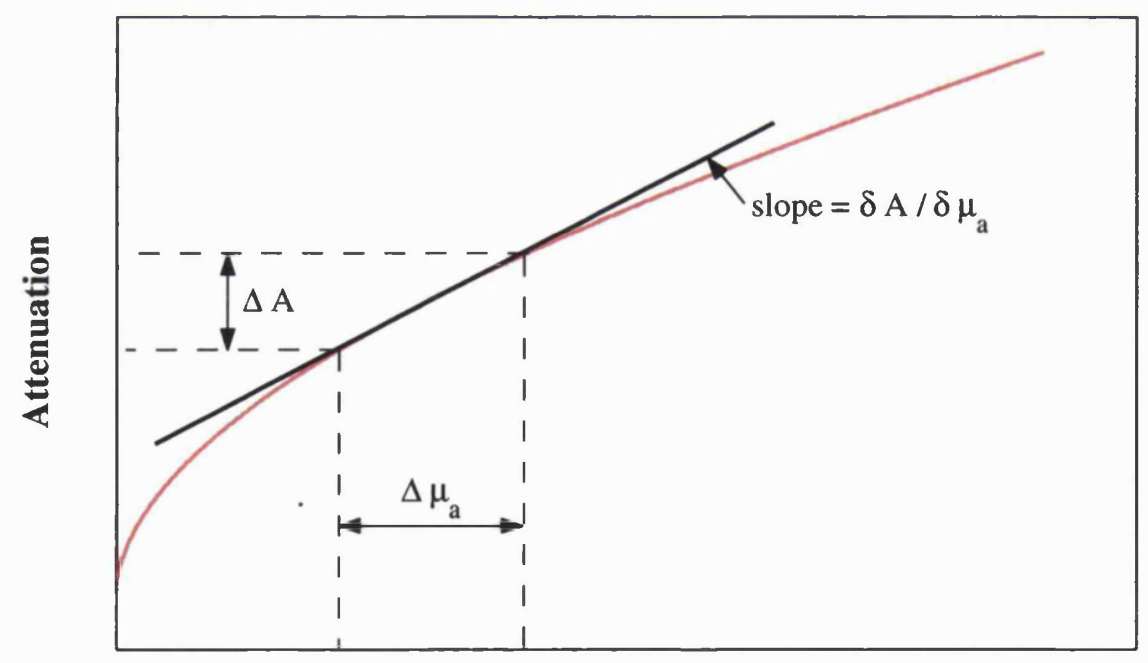

\section{Absorption}

Figure 3.1: The non-linear relationship between attenuation and absorption is approximately linear for small changes in attenuation. The local gradient of the curve represents the mean optical pathlength. 


\subsubsection{The Differential Pathlength}

There are various methods currently employed to determine the DP in tissue. Three such techniques are:

- the measurement of the mean time-of-flight of a picosecond light pulse through the tissue (Delpy et al, 1988; Wyatt et al, 1990; van der Zee et al, 1992)

- the frequency-domain measurement of the phase shift of an intensity-modulated, CW laser beam on passing through the tissue (Chance et al, 1990; Duncan et al, 1993)

- second derivative spectroscopy (Cooper et al, 1996; Matcher et al, 1994), which will be explained in detail in Section 7.1.

As discussed earlier, the time-of-flight and the phase shift methods are essentially the same, the latter being in the frequency domain as opposed to the time domain. The mean time-of-flight of a picosecond light pulse through a scattering medium can be related to the DP as follows

$$
\mathrm{DP}=\frac{c}{n}\langle t\rangle
$$

where $c$ is the speed of light in vacuo, $n$ is the refractive index of the medium and $\langle t\rangle$ is the mean time of flight. Using diffusion theory Arridge et al (1992) have shown that $\langle t\rangle$ is directly related to the gradient of attenuation with respect to absorption. In vivo measurements of the DP in the human adult head, calf and forearm and in the neonatal head have yielded values for the differential pathlength factor (DPF), the DP divided by the source-detector separation, of between 3 and 6, depending on the tissue type (Wyatt et al, 1990; van der Zee et al, 1992). Moreover, it has been shown that the DPF in a given homogeneous tissue is approximately constant above a source-detector separation of 2.5 cm (van der Zee et al, 1992).

In the approximation of Equation (3.6) it is assumed that the DP is constant as $\mu_{a}$ changes. In fact, the DP alters as a continuous function of $\mu_{a}$ (and $\left.\mu_{s}^{\prime}\right)$, therefore a more accurate equality would be

$$
\triangle A=\int_{0}^{\Delta \mu_{a}} \mathrm{DP}\left(\mu_{a}, \mu_{s}^{\prime}\right) \mathrm{d} \mu_{a}
$$

However, for small changes in $\mu_{a}$, particularly at higher values of $\mu_{a}$ (see Figure 3.1), Equation (3.6) is a good approximation of Equation (3.9) and avoids the need for com- 
plex modelling of the DP as a function of $\mu_{a}$ and $\mu_{s}^{\prime}$. This approximation is well suited to NIRS measurements of tissue oxygenation, in which it has been estimated that the oxygen-dependent chromophores are responsible for approximately $30 \%$ of the total absorption coefficient (van der Zee, 1993), hence any changes in oxygenation will produce small changes in $\mu_{a}$ relative to the background tissue absorption.

In full-spectral NIRS measurements an additional factor to consider is the wavelengthdependence of the pathlength (WDPL). The non-linearity of the relationship between attenuation and absorption means that the larger absorption features characteristic of the tissue will appear to be suppressed in the attenuation spectrum, relative to the smaller features. Strictly, therefore, the DP should be determined at each wavelength that $\Delta \mu_{a}$ is required at. For the range 740-840 nm, Essenpreis et al (1993) determined the WDPL in vivo for the adult head, calf and forearm, and also for the infant head (post-mortem). The DPF was seen to decrease with increasing wavelength and varied by about $12 \%$ within the experimental range. Therefore, over small wavelength ranges, the WDPL may be small enough such that a single value for the DP within the range is sufficient for calculations of $\Delta \mu_{a}$. The effect of the WDPL on NIRS algorithms will be discussed in greater detail in Section 7.1.3.

\subsubsection{In Vivo Measurements}

As described in Section 3.1, therefore, the optical properties of tissue can be determined from NIRS measurements by comparison with models of light transport (in the absolute or relative sense) of varying degrees of complexity and validity, or by making linear approximations appropriate to the physical situation (i.e. small changes in $\mu_{a}$ on a relatively large $\mu_{a}$ background are proportional to measured changes in attenuation). However the optical properties are inferred, an important issue is that of tissue heterogeneity within the volume of tissue interrogated by the light. NIRS investigations of the head and limbs, in addition to probing the tissues at depth, i.e. brain and muscle, will also interrogate the skin, skull and adipose tissue layers. If the model or approximation assumes a homogenous medium, the optical coefficients obtained will represent a weighted average of the optical properties of the tissues probed by the light. In measurements of diffuse reflectance, the tissue probed by the light can be described as an essentially 'banana-shaped' volume (Okada et al, 1997), the fatness and average depth of which will depend on the optical properties of the tissue layers and the source-detector separation employed. According to diffusion theory the penetration depth, i.e. the depth at which the photon flux falls by $e^{-1}$ 
of its initial value, is given by

$$
\begin{aligned}
\delta & =\mu_{\text {eff }}{ }^{-1} \\
& =\frac{1}{\sqrt{3 \mu_{a}\left(\mu_{a}+\mu_{s}^{\prime}\right)}}
\end{aligned}
$$

Patterson et al (1995) have derived a simple relationship between the average photon depth $\langle z\rangle$ into the tissue, the penetration depth defined in Equation (3.10) and the sourcedetector separation $\rho$

$$
\langle z\rangle=\frac{1}{2} \sqrt{\rho \delta}
$$

Tissues at different depths will contribute different weights to the average optical properties. Hiraoka et al (1993) introduced the concept of the partial differential pathlength (PDP) to account for the inhomogeneity of the tissue. They determined in simulations of the neonatal head that for an exit angle (relative angle between the fibres) of $45-75^{\circ}$, the PDP in the deep brain tissue is approximately $55-75 \%$ of the total effective pathlength. In general, they conclude that as the source-detector separation increases, the relative contribution of the surface tissues decreases. This conclusion has also been reached in studies of light penetration into skeletal muscle tissue in the limbs (Franceschini et al, 1998). However, the maximum source-detector separation is limited by the SNR, which must be reasonable to enable precise reconstruction of the optical properties. It is important, therefore, to consider the precise nature of the tissues under investigation by in vivo NIRS when reconstructing the optical coefficients.

\subsection{NIRS Steady-State Instrumentation}

As mentioned previously, the experiments in this project involve CW measurements of intensity. The instrumentation described in this section is therefore limited to the basic equipment used for the CW experiments detailed in Chapters 5 and 6 . Figure 3.2 shows a schematic diagram of the generic spectroscopic system employed, each component to be explained briefly as follows.

Light source: The light source (77501, Oriel) depicted in Figure 3.2 is a self-contained unit in which an in-built DC supply powers a 100 watt broad-band quartz-tungsten halogen (QTH) lamp, emitting in the region 250-2500 $\mathrm{nm}$. The lamp output is collimated and re-focused onto an optical fibre, which carries the light to the sample. A coloured-glass 


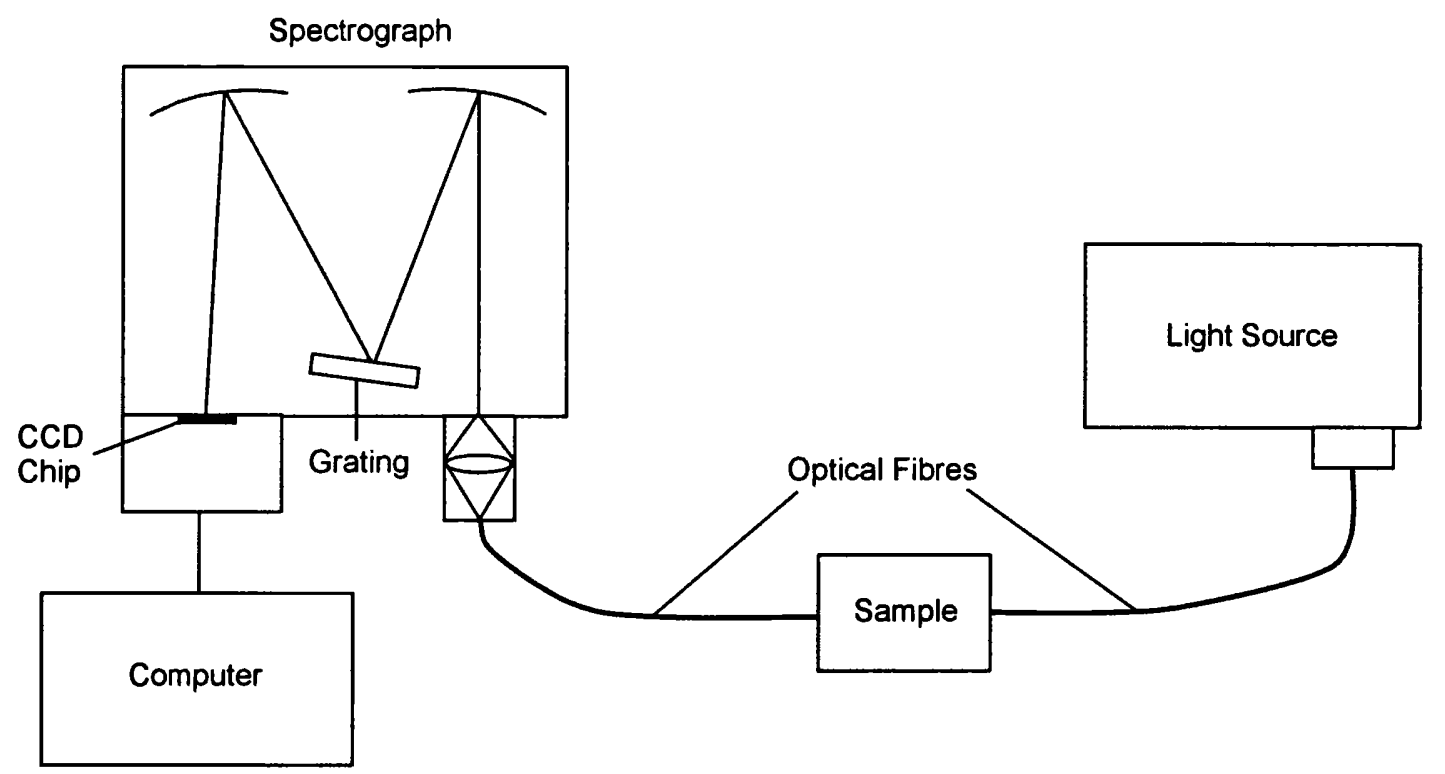

Figure 3.2: Schematic diagram of the generic equipment used for the CW spectroscopic measurements of this project.

filter, fixed within the collimated region of the beam, was used to prevent higher order spectra from the visible wavelengths appearing in the NIR. The most frequently used filter for this purpose was a long-pass filter with a cut-off wavelength ( $50 \%$ transmission) of $610 \mathrm{~nm}$ (RG610, Schott).

Spectrograph and Grating: As shown in Figure 3.2, a second optical fibre collects the light that has passed through the sample and guides it to a spectrograph with a focal length of $27 \mathrm{~cm}$ (270M, Instruments SA). The light is focused on to the variable-width entrance slit by a series of lenses. A collimating mirror on the back wall of the spectrograph directs the beam on to a rotatable diffraction grating (510-14, Instruments SA) with either 600 lines $\mathrm{mm}^{-1}$, blazed (made most efficient) at $750 \mathrm{~nm}$, or 300 lines $\mathrm{mm}^{-1}$, blazed at $1000 \mathrm{~nm}$. The dispersed light is then focused by another mirror on the back wall on to the focal plain of the spectrograph, where the detector is positioned. The spectrograph can also function as a scanning monochromator, with adjustable slits at the exit as well as the entrance. Since broad-band measurements were required, however, the monochromator slits were removed such that the full range of wavelengths determined by the grating dispersion was incident on the focal plain. The spectral resolution is given by the product of the spectral dispersion of the grating and the spectrograph entrance slit width. For example, the 600 lines $\mathrm{mm}^{-1}$ grating has an average linear dispersion of $6.2 \mathrm{~nm} \mathrm{~mm}^{-1}$ in the focal plain, therefore with a slit width of $100 \mu \mathrm{m}$ the spectral resolution would be $0.62 \mathrm{~nm}$. 
CCD Camera: The detector consists of a Peltier-cooled charge-coupled device (CCD) camera (Wright Instruments). The camera head is attached to the spectrograph such that the CCD detector array (CCD30-11, EEV) is in the focal plane of the spectrograph. The CCD array, built on to a silicon chip, has 1024 horizontal and 256 vertical elements, or pixels, each $26 \mu \mathrm{m}$ square. With the 600 lines $\mathrm{mm}^{-1}$ grating, this equates to a pixel bandwidth of $0.16 \mathrm{~nm}$ and a total wavelength range of $165 \mathrm{~nm}$. Photons incident on the array produce photoelectrons which accumulate in the pixels. For a given wavelength, the photon flux is proportional to the number of photoelectrons produced. The charge is transferred down the vertical length of the array to the output register at the bottom of the array. The charge is then moved across the register to the output amplifier and the signal is digitised by a 16-bit analogue-to-digital-converter (ADC). The ADC has a gain setting which alters the number of electrons required to produce one ADC count. Low gain corresponds to 4 electrons per ADC count, high gain to 1 electron per count. The quantum efficiency (QE) of the CCD, i.e. the probability that an incident photon will produce a photoelectron, is determined mainly by the absorption coefficient of silicon, and is significant over a spectral range of $400-1100 \mathrm{~nm}$. At the short wavelength end, the QE is limited by the high absorption of photons in the polysilicate material of the electrodes which cover the surface of the array, preventing the production of photoelectrons in the bulk silicon beneath. The low absorption coefficient of silicon, i.e. a low probability of photon interaction within the device, limits the response at the long wavelength end.

The three main sources of noise associated with a CCD detector are as follows:

i) Photon or shot noise is due to the random nature of the photons incident on the array. Since the production of photoelectrons does not occur at a constant rate, a variation in the current is seen which appears as noise. The random incidence of photons is well described by Poisson statistics (Cope, 1991), therefore the uncertainty in the current produced, the shot noise, is given as the square root of the signal (number of electrons).

ii) Dark current is due to the generation of thermally excited electrons within the device itself. The dark current is reduced by cooling the CCD, falling by a factor of approximately 2.5 for each $10^{\circ} \mathrm{C}$ drop in temperature. The device used in this project has a dark current of $2.4 \times 10^{-4}$ electrons pixel $^{-1}$ second $^{-1}$ at an operating temperature of $195 \mathrm{~K}\left(-78^{\circ} \mathrm{C}\right)$. The dark current noise, i.e. the statistical variation 
in the dark current, is equal to the square root of the dark current signal, as with shot noise.

iii) Readout noise is caused by sources of uncertainty in the camera electronics, also affecting the signal measured by the camera. Its value is in the region of 3-30 signal electrons RMS, but depends on the readout rate, the higher the rate the greater the noise.

In order to determine the signal-to-noise ratio (SNR) of the CCD camera, all sources of noise must be included. Assuming the noise sources listed above are the dominant ones, a simple expression for the SNR of the camera is given by

$$
\mathrm{SNR}=\frac{I \mathrm{QE} t}{\sqrt{I \mathrm{QE} t+N_{d} t+N_{r}^{2}}}
$$

where $I$ is the photon flux (photons pixel ${ }^{-1}$ second $^{-1}$ ), $t$ is the acquisition or integration time (seconds), $N_{d}$ is the dark current (electrons pixel ${ }^{-1}$ second $^{-1}$ ) and $N_{r}$ is the readout noise (electrons RMS pixel ${ }^{-1}$ ). In most cases though, the shot noise is by far the dominant source of noise and readout noise is often ignored.

Computer: An IBM-compatible computer is used as a platform from which the hardware is controlled and the spectral data from the CCD camera can be displayed in real-time and stored. The custom-written software enables the dark-current to be read out and subsequently subtracted from the measured spectra, thus no longer contributing to noise on the measurement. Moreover, a reference spectrum can be acquired and stored that can be used in the calculation of attenuation spectra (see Section 3.1).

\subsection{Optical Coefficients in Whole Tissue}

Having discussed the origins of absorption and scattering in biological tissue in Sections 2.1 and 2.2.2, and the measurement of attenuation in tissue in Section 3.1, this section will present quantitative data for the optical coefficients of various tissues, as found in the literature. In 1990 an extensive review of the optical properties of various tissues was published by Cheong et al (1990), hence the literature search here was limited to work published after this date.

It is important to note the physiological state or condition of the tissue under study, since there can be significant variations in the absolute values of optical coefficients deter- 
mined under in vivo, ex vivo or post-mortem conditions. The main reasons for this are as follows (van der Zee, 1993; Rutishauser, 1994; Zijp and ten Bosch, 1998):

- a lack of blood flow and pressure and hence redistribution of blood in post-mortem tissues

- an influx of ions into the cytoplasm of post-mortem cells, leading to a change in the relative refractive indices of intra- and extracellular fluids and a change in the shape and size of the cells

- reduced oxygenation of tissue haemoglobin due to the continuation of cell metabolism after the oxygen supply has ceased

- drainage of blood from excised tissue samples

- reduced tissue temperature in ex vivo or post-mortem tissues (unless specifically heated to body temperature).

An additional factor to consider in ex vivo experiments is the sample preparation or storage prior to its measurement. Tissue samples are often frozen immediately after excision to prevent decomposition and bacterial contamination, or to aid in the preparation of thin slices. However, the process of freezing and subsequently re-thawing the tissue can itself alter the optical properties. Nau et al (1999) measured the absorption and scattering coefficients of canine prostate tissue and found significantly different results for fresh and previously-frozen samples. They attributed this to cellular damage in the previously-frozen samples during re-heating, due to the expansion of water trapped in the cells on freezing. Having noted these differences, the following presentation of quantitative tissue optical properties will be divided into ex vivo and in vivo measurements. Table 3.1 lists the ex vivo optical properties of various tissues measured at a range of wavelengths. Table 3.2 gives the optical properties determined by in vivo measurements of the intact head and limbs, including the source-detector separation at which they where measured. For some of the measurements of the piglet head (Fantini et al, 1999; Hueber et al, 2001; Kurth et al, 1995) the mean tissue oxygen saturation $\left(\mathrm{S}_{\mathrm{mt}} \mathrm{O}_{2}\right)$ at which the coefficients were determined has been quoted, since these studies were investigating the effect of varying the $\mathrm{S}_{\mathrm{mt}} \mathrm{O}_{2}$ on the optical properties determined. The subsequent sections will briefly discuss the tissue types mentioned and, where possible, show spectra of published optical coefficients determined by ex vivo measurements. 


\begin{tabular}{|c|c|c|c|c|c|c|}
\hline Author & Tissue & Species & $\lambda(\mathrm{nm})$ & $\mu_{a}\left(\mathrm{ODcm}^{-1}\right)$ & $\mu_{s}^{\prime}\left(\mathrm{OD} \mathrm{cm}^{-1}\right)$ & Comments \\
\hline Marchesini et al (1992) & epidermis & human & $400-800$ & $1.04-0.09$ & $13.9-9.1$ & \\
\hline Troy et al (2001) & skin & human & 1000 & 0.43 & 12.7 & $\begin{array}{l}\text { includes epidermis and } \\
\text { dermis, measured at } 37^{\circ} \mathrm{C}\end{array}$ \\
\hline \multirow[t]{2}{*}{ Simpson et al (1998) } & dermis & human & $620-1000$ & $0.05-0.17$ & $12.2-6.9$ & $\begin{array}{l}\text { Caucasian, including epi- } \\
\text { dermis }\end{array}$ \\
\hline & subdermis & & & $0.03-0.08$ & $5.4-4.8$ & \\
\hline \multirow[t]{2}{*}{ Arnfield et al (1992) } & fat & pig & 630,789 & $0.04,0.03$ & $6.7,5.9$ & \\
\hline & muscle & chicken & 630,789 & $0.05,0.03$ & $3.9,3.3$ & \\
\hline Beek et al (1997) & muscle & piglet & 633 & 0.26 & 10.7 & \\
\hline Simpson et al (1998) & muscle & human & $620-1000$ & $0.11-0.61$ & $3.9-2.4$ & $\begin{array}{l}\text { illumination perpendicu- } \\
\text { lar to direction of muscle } \\
\text { fibre }\end{array}$ \\
\hline Firbank et al (1993) & bone & pig & $650-950$ & $0.17-0.22$ & $15.2-10.4$ & \\
\hline Bevilacqua et al (1995) & grey matter & porcine & 670 & 0.29 & 9.1 & \\
\hline \multirow[t]{4}{*}{ van der Zee (1993) } & grey matter & human & $650-1000$ & $0.17-0.56$ & $3.0-1.3$ & neonate \\
\hline & white matter & & & $0.09-0.39$ & $5.6-2.2$ & \\
\hline & grey matter & & & $0.09-0.34$ & $12.2-8.3$ & adult \\
\hline & white matter & & & $0.04-0.26$ & $43.4-32.6$ & \\
\hline Nilsson et al (1997) & blood & human & 633 & 1.30 & 8.3 & \\
\hline Lovell et al (1999) & blood & human & 802 & 0.04 & 13.7 & $\begin{array}{l}\mathrm{HbO}_{2} \text { is replaced by } \\
\text { carbonmonoxide- } \mathrm{Hb}\end{array}$ \\
\hline
\end{tabular}

Table 3.1: NIR optical coefficients of ex vivo tissues. Values are given at specific wavelengths, or ranges indicate the upper and lower limits of $\mu_{a}$ or $\mu_{s}^{\prime}$ within the stated wavelength range. 


\begin{tabular}{|c|c|c|c|c|c|c|c|}
\hline Author & Tissue & Species & $\lambda(\mathrm{nm})$ & $\begin{array}{l}\mu_{a} \\
\left(\mathrm{OD} \mathrm{cm}^{-1}\right)\end{array}$ & $\begin{array}{l}\mu_{s}^{\prime} \\
\left(\mathrm{OD} \mathrm{cm}^{-1}\right)\end{array}$ & $\begin{array}{l}\text { Source-detector } \\
\text { separation }(\mathrm{cm})\end{array}$ & Comments \\
\hline Kurth et al (1995) & head & piglet & 670,830 & $0.10,0.07$ & $3.5,3.3$ & 3 & $55-65 \% \mathrm{~S}_{\mathrm{mt}} \mathrm{O}_{2}$ \\
\hline Fantini et al (1999) & head & piglet & 758,830 & $0.07,0.06$ & $4.0,3.6$ & $1.5-3$ & $\begin{array}{l}\text { multiple source fibres, } \\
60 \% \mathrm{~S}_{\mathrm{mt}} \mathrm{O}_{2}\end{array}$ \\
\hline Hueber et al (2001) & head & piglet & 758,830 & $0.07,0.06$ & $3.0,2.6$ & $1.5-3$ & $\begin{array}{l}\text { multiple source fibres, } \\
58 \% \mathrm{~S}_{\mathrm{mt}} \mathrm{O}_{2}\end{array}$ \\
\hline \multirow[t]{3}{*}{ Doornbos et al (1999) } & forehead & human & 633,700 & $0.04,0.009$ & $7.3,6.7$ & $0.18-1.68$ & multiple detector fibres \\
\hline & arm & & & $0.07,0.04$ & $4.0,3.5$ & & \\
\hline & foot sole & & & $0.03,0.02$ & $4.9,4.1$ & & \\
\hline \multirow[t]{3}{*}{ Matcher et al (1997) } & head & human & 800 & 0.07 & 4.1 & 4 & adult \\
\hline & forearm & & & 0.10 & 3.0 & & \\
\hline & calf & & & 0.07 & 4.08 & & \\
\hline \multirow[t]{3}{*}{ Torricelli et al (2001) } & head & human & $610-1010$ & $0.07-0.16$ & $7.1-4.3$ & 2 & adult \\
\hline & abdomen & & & $0.009-0.09$ & $6.1-3.5$ & & \\
\hline & arm & & & $0.09-0.22$ & $5.3-2.2$ & & \\
\hline Cubeddu et al (1999) & arm & human & 672,818 & $0.12,0.11$ & $4.8,3.9$ & 2 & adult \\
\hline $\begin{array}{l}\text { Farrell and Patterson } \\
\text { (1992) }\end{array}$ & forearm & human & $610-700$ & $0.09-0.01$ & $2.9-2.5$ & $0.1-1.0$ & multiple detector fibres \\
\hline
\end{tabular}

Table 3.2: NIR optical coefficients of in vivo tissues. Values are given at specific wavelengths, or ranges indicate the lower and upper limits of $\mu_{a}$ or $\mu_{s}^{\prime}$ within the stated wavelength range. 


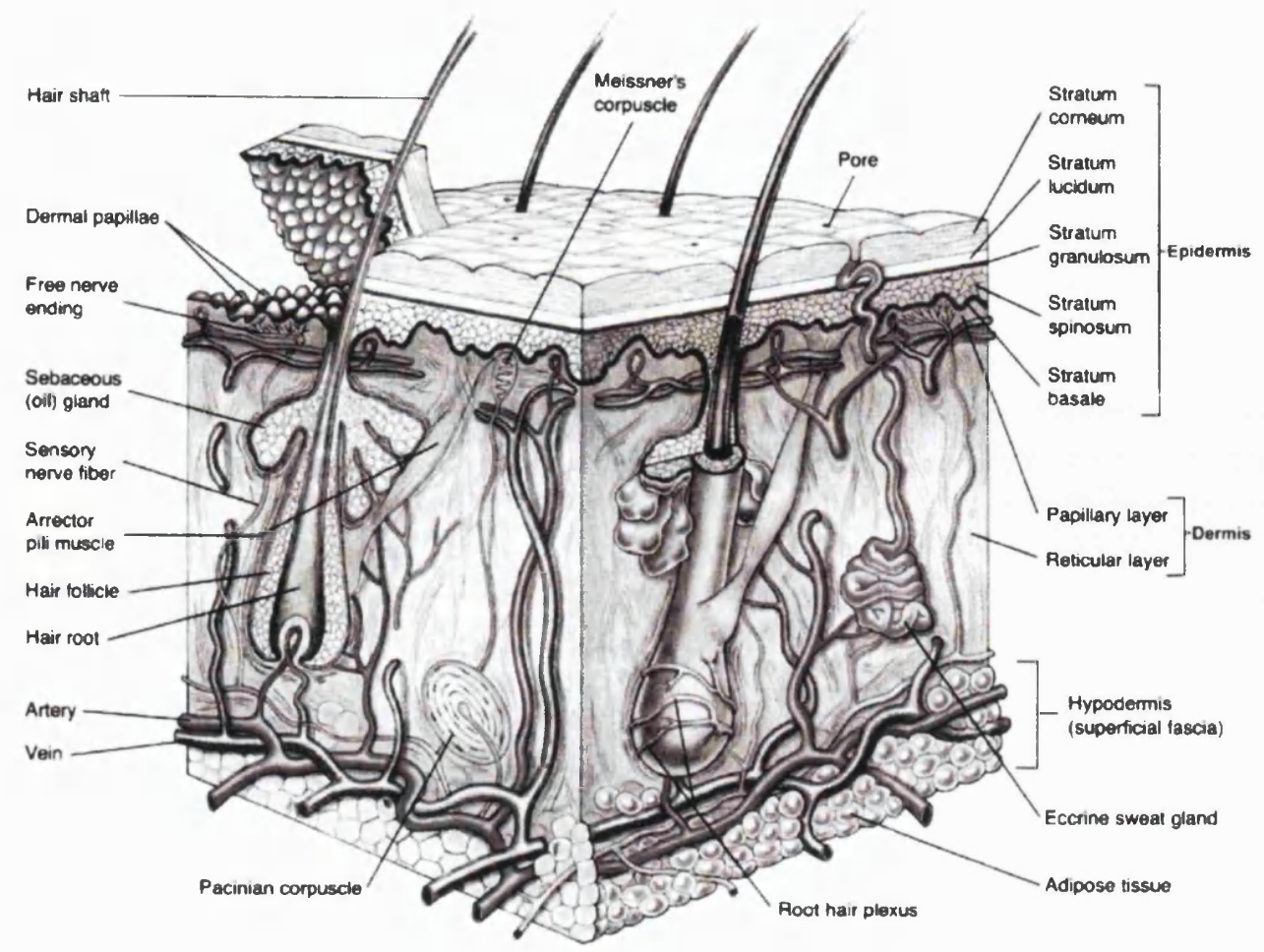

Figure 3.3: Three-dimensional view of the skin (Marieb, 1995).

\subsubsection{Skin}

The optical properties of the skin are important since all in vivo NIRS measurements will include a contribution from the skin. Figure 3.3 shows a three-dimensional view of the human skin and its two main layers: the epidermis and the dermis. Beneath the dermis is the adipose tissue referred to as the hypo- or subdermis, but which is not strictly part of the skin. The dermis is richly vascularised, supplying nutrients to the epidermis which does not contain blood vessels. The extracellular matrix of the dermis contains thick bundles of collagen fibres that give the skin its strength and elastin fibres, which provide elasticity. Figure 3.4 shows the ex vivo absorption and transport scattering properties of Caucasian dermis in the NIR, as measured by Simpson et al (1998).

\subsubsection{Muscle}

The optical properties of skeletal muscle are relevant to in vivo measurements of the forearm (see Section 6.2), which will later be employed in preliminary studies of tissue temperature prediction. The muscles that are attached to bones and are responsible for body movements are comprised of skeletal muscle tissue, illustrated in Figure 3.5 (Marieb, 1995). The muscle cells are known as fibres because of their elongated shape and are 


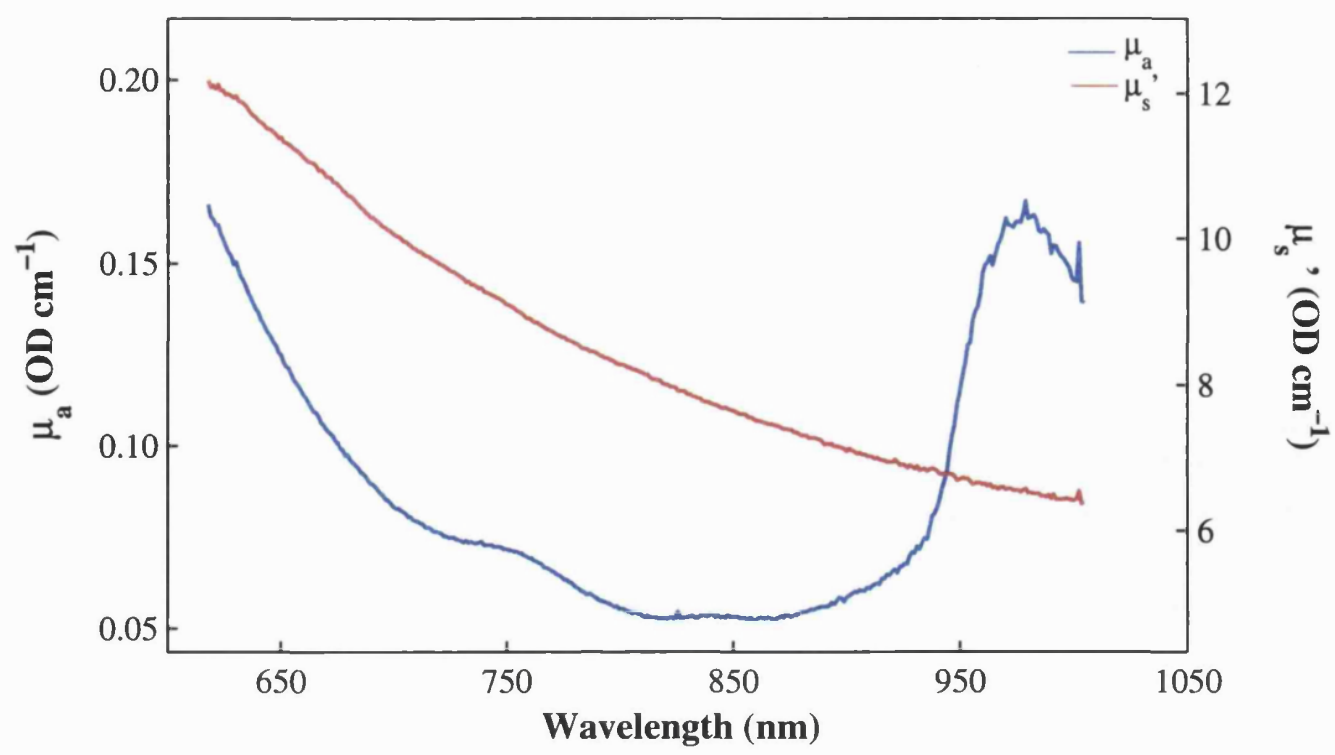

Figure 3.4: Optical properties of ex vivo Caucasian dermis in the NIR (Simpson et al, 1998).

arranged parallel to one another within the muscle. The muscle fibres range in diameter from $10-100 \mu \mathrm{m}$ and are grouped into larger bundles which are bound by a collagenous tissue. Muscles are well-supplied with blood by vessels that infiltrate the fibre bundles to serve each individual fibre. Within the muscle cells themselves is a protein known as myoglobin. As mentioned in Section 2.1.5, myoglobin stores oxygen within the muscle fibre and has a similar absorption spectrum to haemoglobin. In modelling light transport through tissue, e.g. using Monte Carlo simulations (see Section 2.3), it is usually assumed that the scattering volumes are randomly aligned with respect to one another and hence no fixed phase relationship exists between the individual scattered fields. In muscle this is not the case since the muscle fibres have a definite orientation, therefore scattering

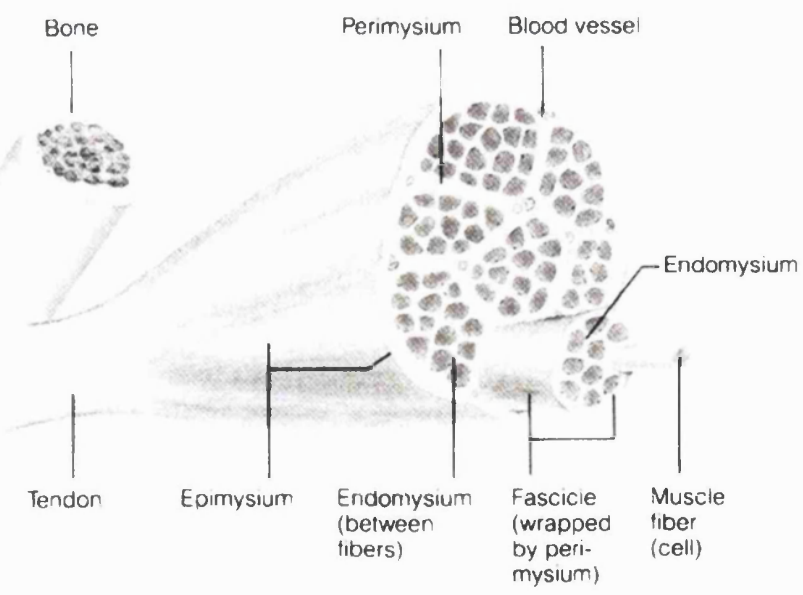

Figure 3.5: Human skeletal muscle (Marieb, 1995). 


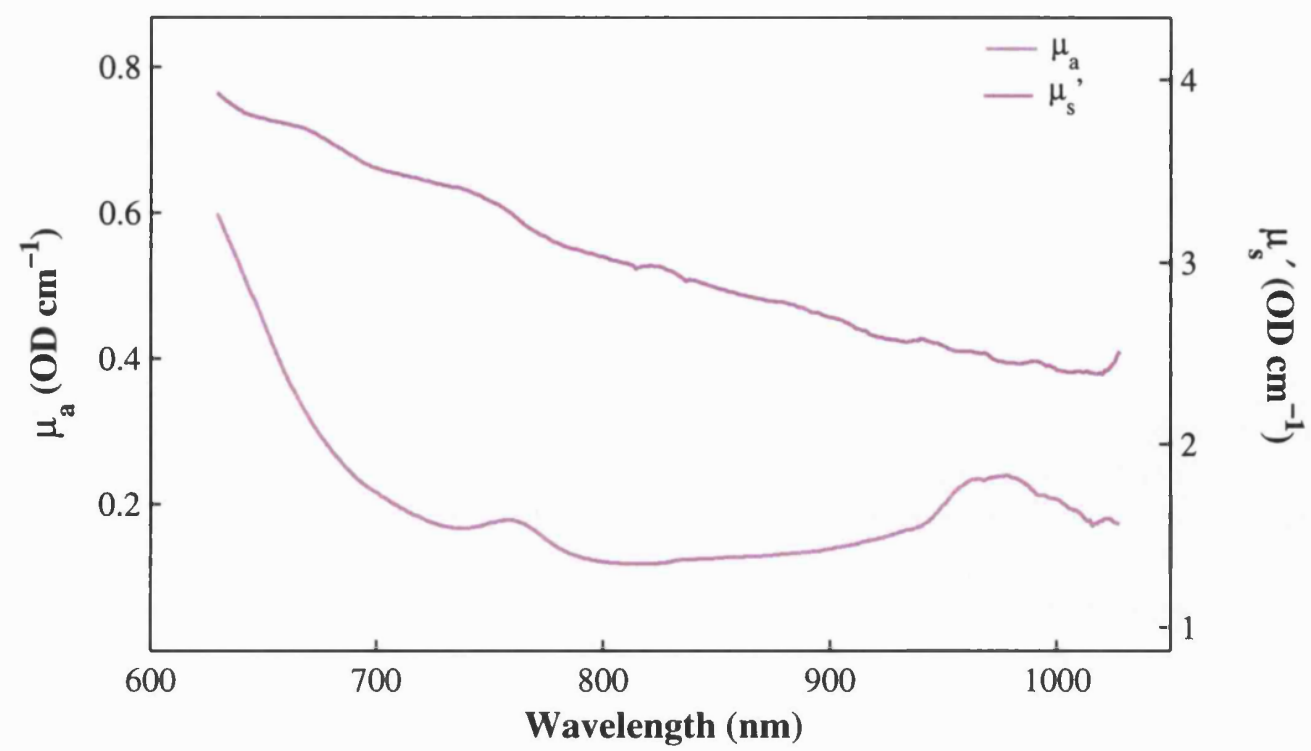

Figure 3.6: Optical properties of ex vivo human abdominal muscle in the NIR (Simpson et al, 1998).

within the muscle will not be isotropic and the total scattered field will depend on the direction of illumination (Zijp and ten Bosch, 1998). Figure 3.6 shows the ex vivo optical properties of human abdominal muscle tissue (Simpson et al, 1998), in which the direction of illumination was perpendicular to the direction of the muscle fibres.

\subsubsection{Bone}

Of the different types of bone, the flat bones of the skull and the long bones of the limbs are of particular interest to NIRS measurements. Figure 3.7 shows a cross-section through

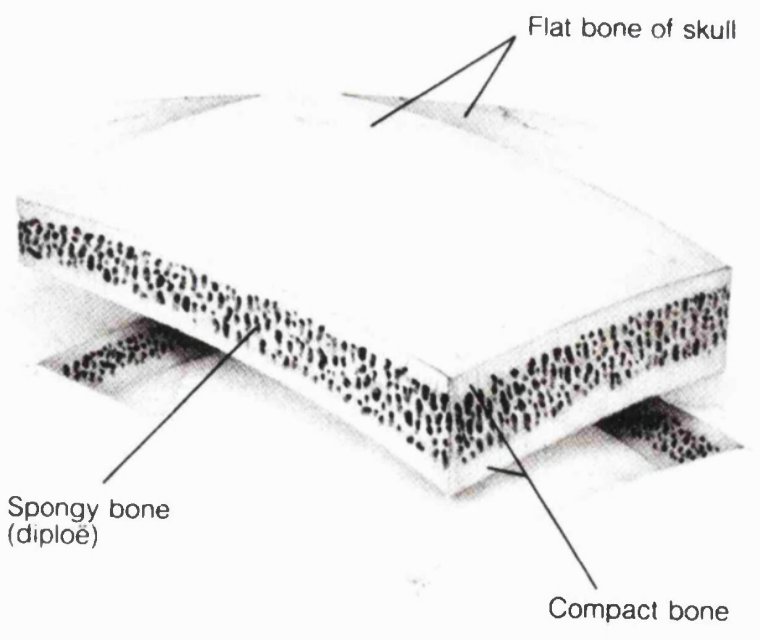

Figure 3.7: Cross-section of flat bone in the human skull (Marieb, 1995). 


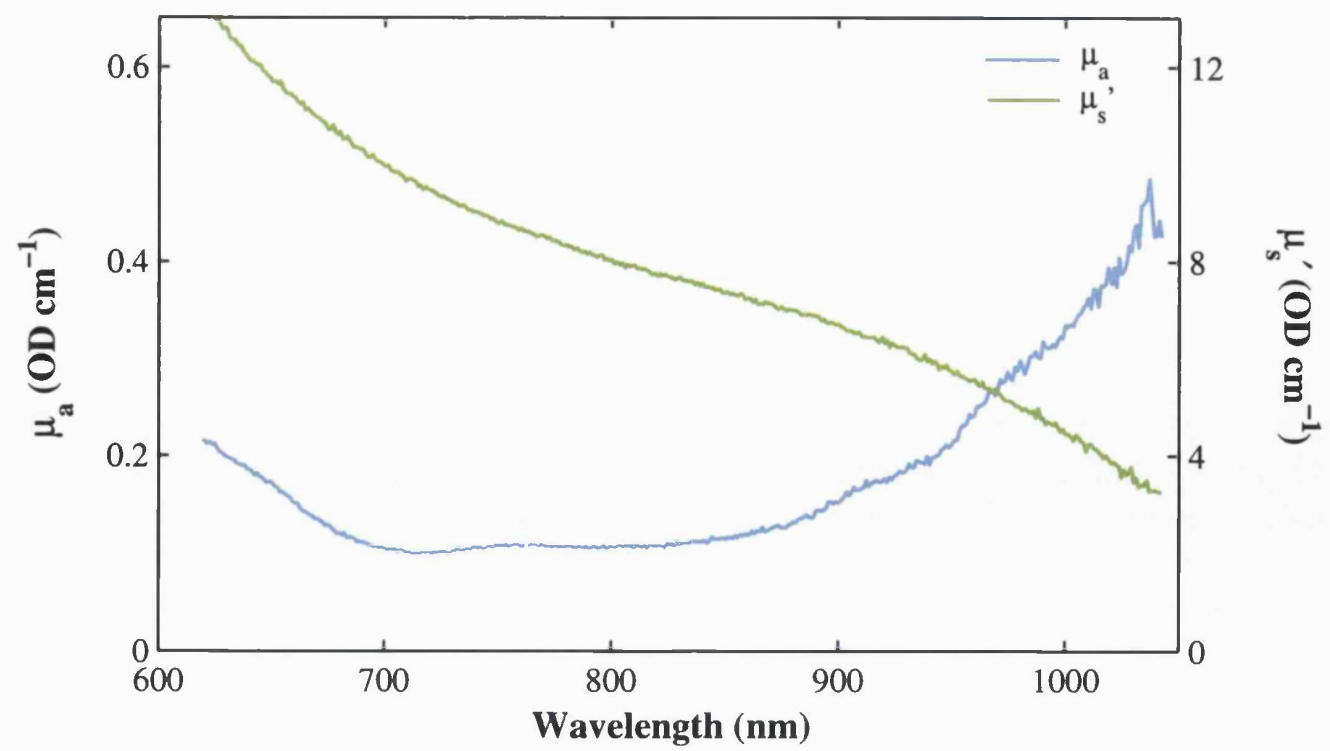

Figure 3.8: Optical properties of ex vivo porcine skull in the NIR (Firbank et al, 1993).

the flat bone of the skull, which comprises of two types of bone: cortical and spongy bone. The cortical bone is dense and hard and the spongy bone is comprised of tiny struts of bone called trabeculae between which bone marrow exists. Bone is a highly scattering medium because of the minerals present in its extracellular matrix. In the new-born infant mineralisation of the skull bones is incomplete, hence one would expect the scattering coefficient of the neonatal cranium to be less than that of the adult. Light absorption by bone will be due to haemoglobin in the blood vessels and also due to red marrow (where red blood cells are formed), of which there is a greater proportion in the bones of the new-born compared to the adult. Figure 3.8 shows the ex vivo NIR optical coefficients for porcine skull (Firbank et al, 1993).

\subsubsection{Brain}

Nervous tissue in the brain is comprised of two cell types: nerve cells known as neurones and supporting cells known as neuroglia. A typical neurone is illustrated in Figure 3.9. The nerve cell bodies and their axons respectively form the main components of the grey and white matter in the brain. Scattering in the brain is mostly attributable to the presence of lipids, which, as discussed in Section 2.1.3, also contribute to the total absorption coefficient. The lipid content in the white matter is considerably greater than in the grey matter, with values for the adult brain of 18.1 and $5.3 \%$ respectively (Woodard and White, 1986). This is largely due to the lipid-rich sheath that covers nerve axons in the white matter known as myelin, which protects and electrically insulates the axons. The myelin 


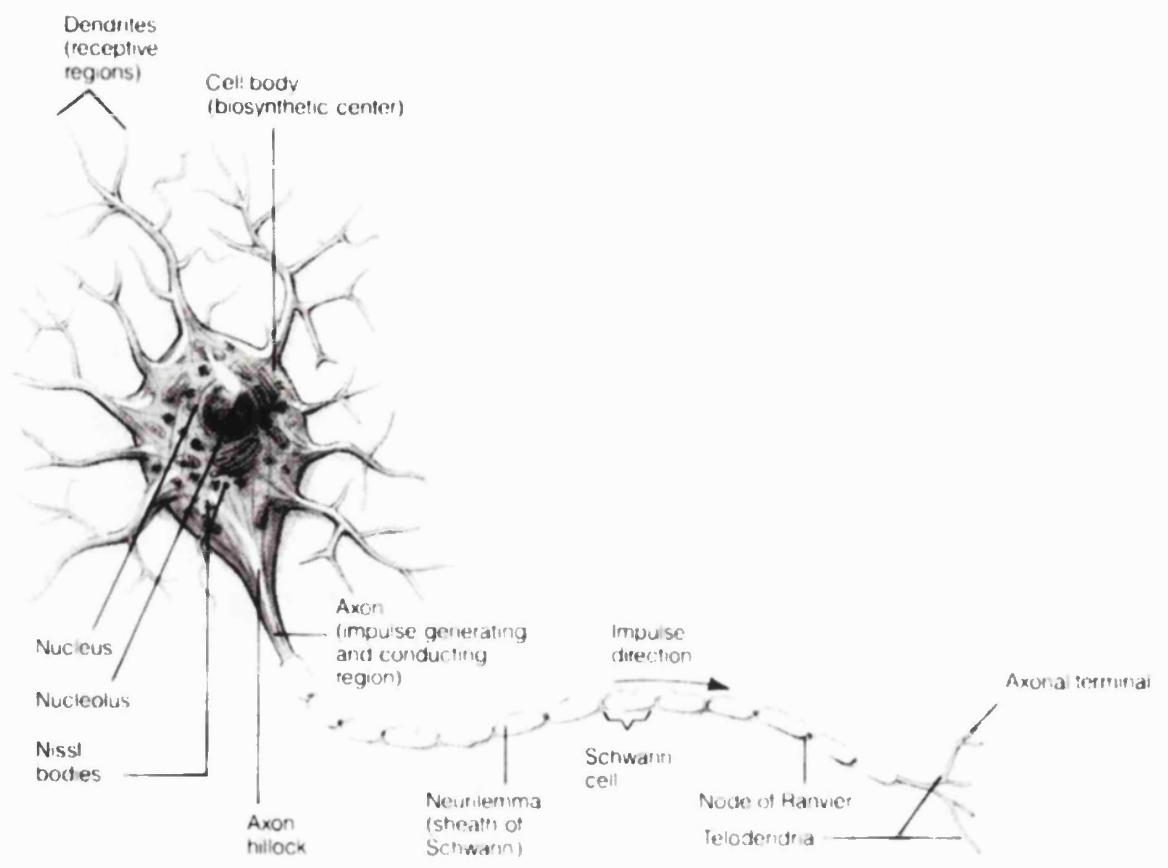

Figure 3.9: A typical neurone present in the CNS.

sheath forms in segments around the nerve fibres, greatly increasing the transmission of electrical impulses down the length of the fibres. Due to the high refractive indices of the lipids in the myelin, white matter has a significantly higher scattering coefficient than grey matter, as indicated in Table 3.1 (van der Zee, 1993; Bevilacqua et al, 1995). Scattering in the brain increases from birth as lipid content increases due to myelination, indicated in Table 3.1 by the difference between optical coefficients in the neonatal and adult brain.
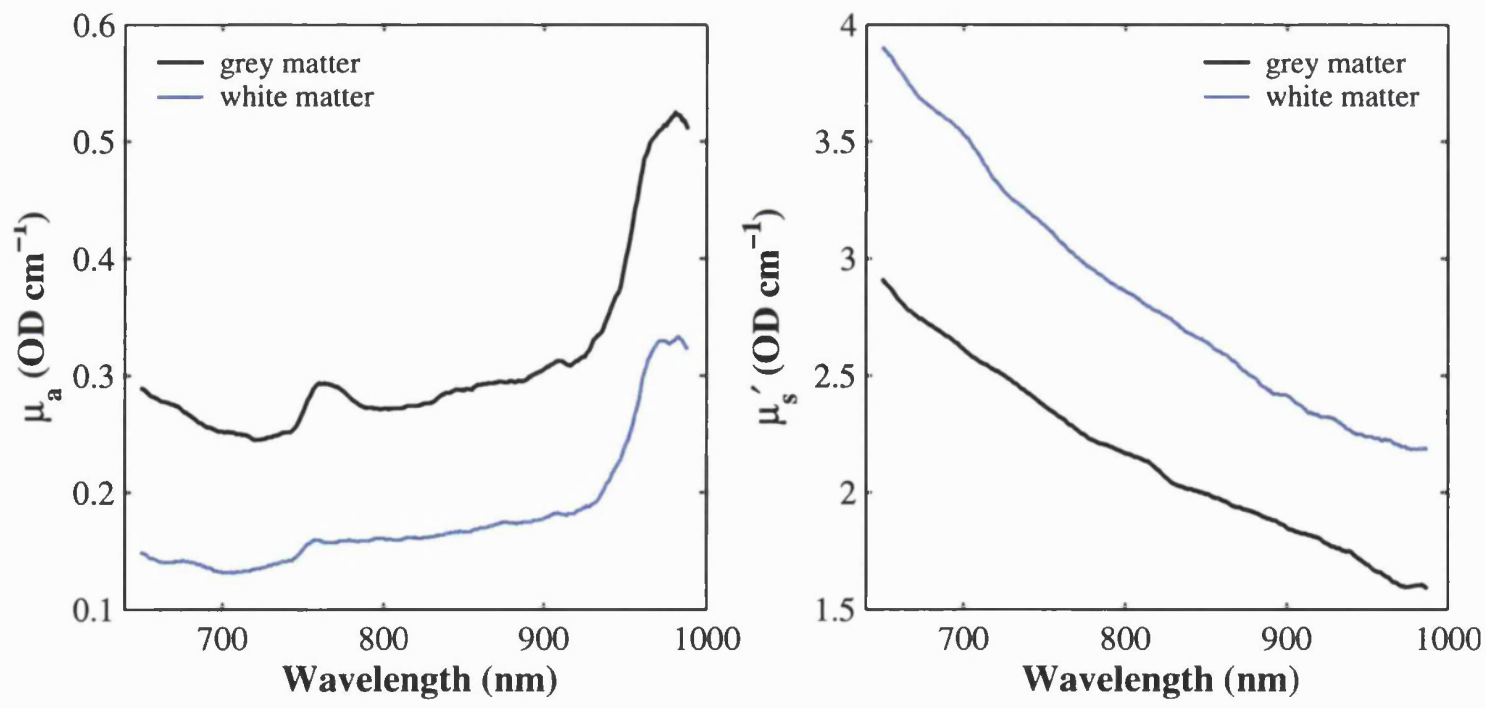

Figure 3.10: Optical properties of post-mortem neonatal brain matter at 40 weeks gestational age in the NIR. Left: Absorption for grey and white matter. Right: Scattering for grey and white matter 
Figure 3.10 shows the ex vivo optical properties for grey and white brain matter of a post-mortem neonate at 40 weeks gestational age (van der Zee, 1993).

\subsubsection{Blood}

Whole blood consists of roughly $55 \%$ plasma and $45 \%$ formed elements. Red blood cells are by far the most abundant of the formed elements, the others, leukocytes and platelets, contributing less than $1 \%$ to whole blood volume. Haemoglobin molecules in the red blood cells are responsible for almost all of the absorption of light by blood (see Section 2.1.2). Scattering in blood is due to the refractive index mismatch between plasma and the red blood cell membranes (see Section 2.2.2). Table 3.1 includes the optical coefficients of whole blood, measured at approximately $25-28^{\circ} \mathrm{C}$ (Nilsson et al, 1997 ; Lovell et al, 1999). The sample preparation and measurement procedure described by these two studies were sufficiently different that, even at comparable wavelengths, the determined optical coefficients are unlikely to be comparable. For example, to prevent blood clotting during the experiment Lovell et al (1999) removed the plasma from the blood and replaced it with a phosphate buffered saline solution containing heparin, an anti-clotting agent. Moreover, in order to apply diffusion theory to their measurements, Lovell et al converted the oxyhaemoglobin in the red blood cells to carboxyhaemoglobin ( $\mathrm{HbCO}$ ), which they reported has an absorption coefficient that is approximately $5 \%$ of that of $\mathrm{HbO}_{2}$ in the NIR. Nilsson et al (1997), on the other hand, did not replace or dilute the plasma with saline, although an anti-coagulant was added, and they attempted to maintain the oxygen saturation of haemoglobin at a constant level during the experiment. 


\section{Chapter 4}

\section{Methods of Tissue Temperature Measurement}

In this chapter various techniques for the non-invasive monitoring of tissue temperature are reviewed, including the proposed NIRS method, highlighting their relative advantages and disadvantages. The emphasis is on the new-born human brain, since, as discussed in Section 1.1, one of the major applications of the work developed in this project is the intended monitoring of cerebral temperature during hypothermic treatment in birthasphyxiated infants.

Section 4.1 discusses the definition and measurement of core body temperature by conventional and other non-invasive methods. It can also be important to measure temperature in a particular part of the body or even a specific volume of tissue. As discussed in Section 1.2.3, one example of this is the case of selective head cooling, in which cerebral temperature may significantly differ from that in the rest of the body. Therefore, Section 4.2 describes methods in which non-invasive temperature measurement can be applied to localised tissue areas, again with particular focus on the infant brain. Finally, Section 4.3 discusses the feasibility of using NIRS to monitor tissue temperature non-invasively, describing how temperature affects the optical properties of tissue and reviewing studies in which the temperature-dependence of NIRS tissue measurements is investigated. 


\subsection{Measurement of Core Body Temperature}

Deep or 'core' body temperature refers to that of the tissues deep within the skull or the thoracic and abdominal cavities, whereas surface or 'shell' temperature refers to the outer body tissues, essentially the skin. Core body temperature is regulated mainly by the hypothalumus in the brain, which responds to input from thermoreceptors located centrally in the body core and peripherally in the skin. The pulmonary artery is considered to be the 'gold standard' measurement site for core body temperature (Fulbrook, 1997). However, the use of this site is rarely an option in normal clinical practice, and other methods of temperature measurement are generally employed. There has been much debate about the most reliable site from which to monitor body temperature in children (Haddock et al, 1996; Robinson et al, 1998; Craig et al, 2000). The majority of the studies conclude that axillary temperature measurement (from the armpit), whilst safe and easy to perform, is not sensitive enough to detect fever in infants, and hence rectal temperature measurement should be used. Rectal temperature has most commonly been monitored by conventional mercury-glass thermometers. The use of mercury thermometers in neonatal wards has, however, also been under recent debate, due to safety and cost issues (Sganga et al, 2000), and the length of time required to achieve an accurate measurement (Craig et al, 2000). Sganga et al (2000) condone the use of digital thermometers in healthy neonates as a cheaper alternative to mercury-glass thermometers without the associated risks. Whilst these methods can provide clinicians with a more or less non-invasive measurement of body temperature, they do not lend themselves to measurement of temperature within a particular area of the body, e.g. the brain. The following sub-sections describe two other methods of measuring core temperature non-invasively, which have specifically been applied to monitoring cerebral tissue temperature: tympanic thermometry and the zero heat flow method.

\subsubsection{Tympanic Membrane Thermometry}

In recent years the tympanic membrane has become a popular temperature measuring site, in particular with the use of infrared thermometry (Nobel, 1992). The tympanic membrane (TM) is the boundary between the outer and middle ears, commonly known as the eardrum. Due to its close proximity to the carotid artery, temperature at the TM should mirror core body temperature well. Ferrara-Love (1991) reports no significant difference between temperatures measured in post-operative patients using a TM thermometer and 
via a pulmonary artery catheter. TM temperature is also thought to accurately represent core cerebral temperature (Childs et al, 1999), which could differ from core trunk temperature, e.g. during brain hypothermia.

An extensive number of studies have compared brain and TM temperatures by a variety of methods under differing conditions. Mariak et al (1994) compared invasive brain temperature measurements with those made at other externally accessible sites during open-brain surgery in humans. They concluded that TM temperature gave the best approximation of the average cerebral temperature. During mild hypothermia $\left(35^{\circ} \mathrm{C}\right)$ in piglets, Haaland et al (1996) reported that TM temperature was a better indicator of brain temperature than colonic temperature. However, they noted that during moderate hypothermia $\left(29^{\circ} \mathrm{C}\right)$ and rapid changes in temperature the tympanic-cerebro correlation was poorer, an observation also made by Stone et al (1995) during studies of heart surgery patients. There are further studies that conclude that TM temperature does not give a good indication of changes in brain temperature (Shiraki et al, 1988). Sato et al (1996) suggest that the discrepancy between core and TM temperatures is due to poor thermal insulation of the measuring probe from the surrounding ear canal and poor contact between the probe and the membrane itself. Contact thermistors or thermocouples are not, however, suitable in the majority of clinical cases and have associated risks such as injury to the delicate TM and the spread of infection.

Infrared tympanic thermometry (ITT), which has become increasingly popular in hospitals since the late 1980's, has been found to provide fast, accurate temperature readings at relatively low cost (Shinozaki et al, 1988). The non-contact thermometers detect infrared radiation emitted from the TM and calculate an equivalent black-body temperature. Since the TM is optically insulated from the outside environment, due to the shape of the ear canal, it can be approximated as a black-body. In a comparison study between contact and non-contact (i.e. infrared) TM temperature measurements, Terndrup et al (1997) indicated that the infrared thermometer gave an accurate estimate of TM, and hence core, temperature in healthy children. In contrast, many neonatal and paediatric studies have shown infrared tympanic thermometers (ITTs) to be inaccurate, or insensitive to temperature changes, when compared to other core-estimating techniques (Muma et al, 1991; Yetman et al, 1993; Lanham et al, 1999). Childs et al (1999) suggest that conflicting results from studies evaluating the accuracy of ITTs could mainly be due to probe positioning. If the sensor is not correctly positioned it may detect radiation emitted from the ear canal and not the TM. Since the ear canal is not in equilibrium with the arterial 
supply and is more readily affected by environmental temperature this may give rise to an erroneous reading of core body temperature.

A further point made by Shibasaki et al (1998) is that current ITTs are not capable of monitoring temperature continuously, which is especially important in the case of hypothermic treatment. They have developed a method of continuously measuring TM temperature using an optical fibre and infrared detector, which showed no significant differences from TM temperature measured by a contact thermistor during exercise and face fanning. The adult volunteers gave an indication of when they believed the fibre to be close to the TM, based on the amplitude of the sound, which travelled down the fibre, of a mechanical chopper used in the set-up. As the method still involves insertion of the probe (the fibre in this case) into the ear canal, however, it is unlikely to be suitable for continuous monitoring of brain temperature in new-born infants.

\subsubsection{The Zero Heat Flow Method}

Another method of measuring core temperature is based on the 'zero heat flow' principle. The assumption is made that if heat loss from the surface of the body is reduced to zero, the gradient between core and surface temperature will also tend towards zero. When an equilibrium has been reached there should be a region of tissue below the surface which will be at a uniform temperature. The depth to which the tissue reaches a uniform temperature depends on the size of the area over which the surface is insulated. A number of studies have reported the use of the zero heat flow method in monitoring tissue temperature in new-born infants. These studies have taken advantage of the high insulation properties of the infant mattresses used in neonatal wards and intensive care incubators in order to achieve the zero heat flow condition. Simbruner et al (1994) investigated the relationship between brain temperature and cerebral metabolism or circulation in neonates, using the zero heat flow method to determine temperature within the head. The zero heat flow condition was achieved by turning the head to one side and positioning the monitoring thermistor between the infants temple and the incubator mattress. They believed the method to be accurate and precise enough to determine small temperature differences between infants with normal or abnormal cerebral tissue or haemodynamics, although they also suggested a validation of these results with a more direct or invasive method.

The Deep Body Thermometer (DBT), first developed by Fox and Solman (1971), achieves the zero heat flow condition by heating the skin surface such that outward heat flow from the core is matched by inward heat flow from the surface. The thermometer 


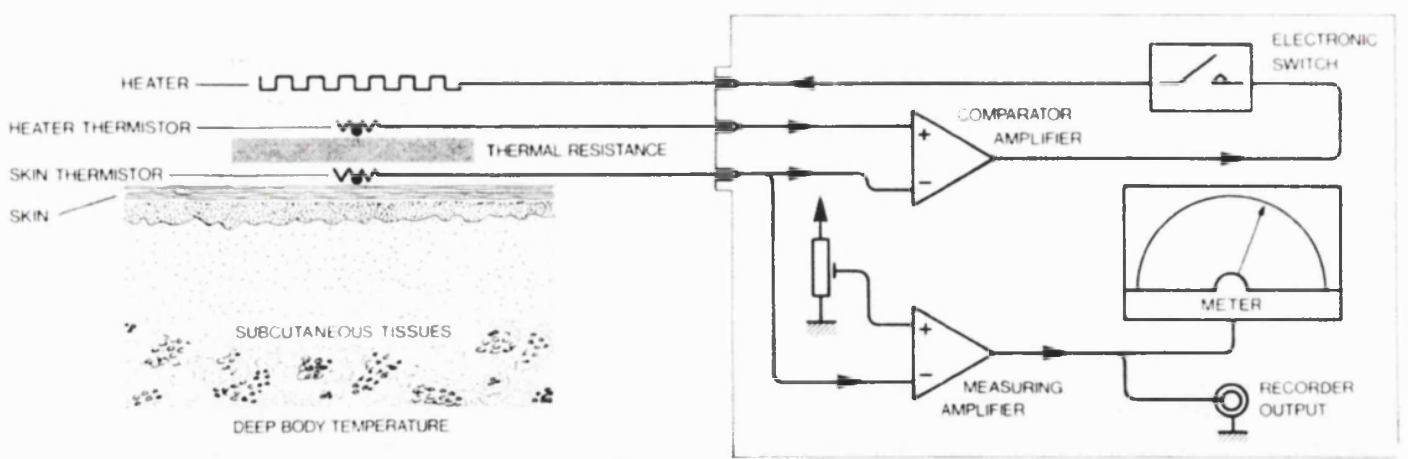

Figure 4.1: Schematic diagram of the deep body thermometer probe, based on the zero heat flow method, developed by Fox and Solman (1971).

probe, shown in Figure 4.1 (Solman and Dalton, 1973), is approximately $6 \mathrm{~cm}$ in diameter and consists of two temperature sensors separated by an insulating pad, which also houses a heating element. The temperatures of the sensors, one of which is in contact with the skin surface, are compared by an operational amplifier, the output of which controls the heating element, switching it on or off depending on the direction of the temperature gradient between the two sensors (Solman and Dalton, 1973). When the sensors eventually reach the same temperature, no additional heat can flow out through the pad and the 'deep body' temperature is inferred by the temperature of the skin sensor.

Following its development some thirty years ago, many studies were done to assess the accuracy of the DBT. In a study by Singer and Lipton (1975), a close correlation was found between the temperature measured by the DBT, applied to the upper sternum, and the temperature of the tympanic membrane. The upper sternum has been recommended as a suitable measuring site (Fox and Solman, 1971) since it is relatively flat, has little subcutaneous fat and is close to the large systemic blood vessels which will be at core temperature. Togawa (1979) comments that the forehead is also a convenient measuring site for the thermometer probe and cites findings from a study by Tsuji et al (1976) in which temperature measured at the forehead by the DBT probe was seen to correlate closely to blood temperature in the jugular vein. A modified probe, developed by Togawa et al (1976), incorporates an aluminium casing (approximately $2 \mathrm{~cm}$ in diameter) around a smaller version of the original probe, which minimises radial heat flow from the probe centre and thus improves the accuracy of the DBT. The DBT response time, i.e. the time taken for the initial equilibrium temperature to be reached, is reported to be between 15 and 20 minutes (Togawa, 1985), or slightly less if the probe is allowed to heat up to $37^{\circ} \mathrm{C}$ prior to application (Solman and Dalton, 1973). The temperatures measured by this probe 
have been shown to reflect the temperature at a depth of $18 \mathrm{~mm}$ or greater below the skin surface, i.e. the core temperature (Matsukawa et al, 1996).

A relevant point to consider is whether the zero heat flow method (achieved via the DBT or other method) could be used to monitor tissue temperature during mild hypothermia. In a study of patients undergoing cardiopulmonary bypass and moderate hypothermia $\left(25-28^{\circ} \mathrm{C}\right)$, the modified DBT was used to monitor deep brain temperature at the forehead (Muravchick, 1983). The author concluded that the DBT was not suitable for monitoring the rapid changes in temperature seen during cardiopulmonary bypass. This hypothesis was supported in a review by Togawa (1985), who suggests that zero heat flow measurement at either the forehead or the torso is a reliable method of measuring deep body temperature except during rapid cooling or warming. Conversely, in a recent study (Harioka et al, 2000), temperature monitored by the DBT at the (adult) forehead was seen to correlate well with blood temperature measured in the pulmonary artery during intraoperative mild hypothermia $\left(\sim 34^{\circ} \mathrm{C}\right)$, although the authors noted that the probe was allowed to equilibrate for 20 minutes prior to induction of anaesthesia. Dollberg et al (1993) point out that the zero heat flow method relies on heat conduction to the surface, but that heat is also transferred by the circulation and the two forms of heat flow are not independent. Thus if heat flow via the circulation were to change during hypothermia, for example due to vasoconstriction, this would affect heat flow to the surface and hence the time response of the measuring system. A potentially serious hazard is the possibility that the imposed heat insulation at the skin surface may in fact alter cerebral tissue temperature during the treatment (Togawa, 1979). Gunn and Gunn (1996) investigated the effect of a radiant heater on the temperature gradient in the head of new-borns in a neonatal intensive care unit, using a similar zero heat flow method to Simbruner et al (1994). They had expressed concern that since brain temperature depends partly upon radiative heat loss from the scalp, which in turn depends on skin and ambient temperatures, the use of overhead heaters could in fact reduce the natural core to surface temperature gradient in the head, thus increasing brain temperature. It has been shown, in adult rats, that an increase in body temperature of just a few degrees can significantly affect the extent of neuronal damage following an ischaemic insult (Baena et al, 1997). In their study Gunn and Gunn (1996) found no significant differences between core head and core body temperatures (as measured by the zero heat method) and rectal temperature when the overhead heater was switched on, although scalp temperature was seen to rise sharply. They concluded that the subsequent reduction in core to surface temperature gradient in 
the head must affect the temperature of intermediate or superficial brain structures. This alteration in temperature of the surface tissues would not be measured by the zero heat flow method, as indicated by the findings of the study (Gunn and Gunn, 1996), since it represents the deep body or head temperature.

\subsection{Non-Invasive Brain Temperature Measurement}

This section will discuss non-invasive methods of brain temperature measurement that are currently being researched and developed world-wide in academic and industrial institutions. These include microwave radiometry, magnetic resonance thermometry and ultrasound thermometry. Many studies based on these methods have investigated the application of remote thermometry to hyperthermic oncological treatments (Carter et al, 1998; Dubois et al, 1993). The discussion here, however, will focus if possible on thermometry in hypothermic therapies, in particular in the neonatal brain. The advantages and disadvantages of the methods, with regard to the specific application, are summarised.

\subsubsection{Microwave Radiometry}

The technique of microwave radiometry in the application to non-invasive thermometry is based on the detection of electromagnetic radiation in the microwave region emitted from a 'hot' body (Foster and Cheever, 1992; Leroy et al, 1998). Any material body at a temperature above that of absolute zero will emit thermal radiation, the spectrum of which is directly related to the temperature of the body. For living human tissue, at a temperature of around $37^{\circ} \mathrm{C}$, spectral emission is most intense in the mid-infrared at about 9.7 $\mu \mathrm{m}$ (Togawa, 1985). At a microwave frequency of $3 \mathrm{GHz}(10 \mathrm{~cm}$ wavelength), a typical frequency used in microwave radiometry (Leroy et al, 1998), the emitted intensity is approximately $10^{8}$ times less than that at the peak (Togawa, 1985). However, with sensitive enough equipment this radiation can be detected and a temperature resolution of $0.1^{\circ} \mathrm{C}$ achieved (Edrich et al, 1980). At microwave wavelengths the penetration depth through human tissue is the order of several centimetres, much deeper than the few millimetres or less characteristic of the mid-infrared (Godik and Gulyaev, 1991). Spatial resolution is, however, compromised by the use of longer wavelengths: at a wavelength of $3 \mathrm{~cm}$ the spatial resolution has been reported to be about $2 \mathrm{~cm}$ (Edrich et al, 1980).

Microwave radiometers detect the average power emitted from a particular volume of the medium beneath the detecting antenna. The received signal depends on many 
factors, such as the temperature distribution in the measured volume and its dielectric properties (Cheever and Foster, 1992). The effective depth of sensing, i.e. the depth at which the signal is $e^{-1}$ that at the surface, depends on the antenna geometry: a larger antenna increases the effective depth at the cost of resolution (Cheever and Foster, 1992). The radiometric temperature (known as the brightness temperature) is determined by a complex two-step process (Leroy et al, 1998). Initially, the received radiation field pattern of the antenna is estimated by assuming it to be the same as the absorbed field distribution seen in the medium were the antenna to be used to transmit energy into the medium (Foster and Cheever, 1992). Subsequently, the temperature is found by applying an inversion process to the radiometric data, which has been weighted by the antenna field distribution. There are many inversion techniques used for recovery of the temperature distribution in biological tissue (Foster and Cheever, 1992). Some require the use of multifrequency radiometers (Mizushina et al, 1992), whilst others depend on a fixed-frequency, multi-angle method (Montreuil and Nachman, 1991).

Gustov et al (1985) investigated the non-invasive measurement of cranio-cerebral temperatures in healthy adults by the use of microwave radiometry. The system they used was reported to measure temperature to an accuracy of $0.1^{\circ} \mathrm{C}$. In three patients, brain temperature at a depth of 5-6 cm was measured directly during surgery. The radiometric temperature measured at the corresponding site prior to surgery was found to differ from brain temperature at depth by only $0.4-0.8^{\circ} \mathrm{C}$. Recently, an interest in the application of microwave radiometry to monitoring neonatal brain temperature during hypothermic treatment has arisen (Hand et al, 2001).

Foster and Cheever (1992) discuss the relative advantages and disadvantages of microwave thermometry. In their opinion, the advantages include the comparative simplicity of the hardware, e.g. in comparison to magnetic resonance systems (see Section 4.2.2), and the potential for greater tissue-depth temperature determination compared to infrared thermomgraphic techniques. Another obvious advantage is the inherent safety of the technique, since no external field is applied to the tissue. Amongst the disadvantages, Foster and Cheever (1992) include the relatively poor temporal and spatial resolution of the measurements, the susceptibility to interference from other electrical signals and the possibility that the radiometric signal may reflect temperature-dependent changes in tissue dielectric properties as well as tissue temperature changes. 


\subsubsection{Magnetic Resonance Thermometry}

The application of nuclear magnetic resonance (NMR) to non-invasive tissue thermometry is based on the effects of temperature on several physical parameters that influence the NMR signal. These include the water proton chemical shift (Cady et al, 1995; de Poorter et al, 1995), the spin-lattice relaxation time (Young et al, 1994; Bertsch et al, 1998) and the water diffusion coefficient (Delannoy et al, 1991).

The water proton chemical shift is the shift in the proton resonance frequency due to a change in the magnetic field strength local to the proton (Eisenberg and Kauzmann, 1969). Hindman (1966) studied the effect of temperature on the proton chemical shift in water, suggesting that the observed shift was due to the temperature dependence of the electronic environment of the proton. Chemical shifts must be measured with respect to some reference resonance (Eisenberg and Kauzmann, 1969). In magnetic resonance spectroscopy (MRS) the proton chemical shift of water with changing temperature has been measured relative to that of the metabolite methyl $\mathrm{N}$-acetyl aspartate (Cady et al, 1995). In their study, Cady et al (1995) investigated this temperature-dependent change in the brains of anaethetised, ventilated new-born piglets, and correlated it to TM temperature measured by a contact thermistor. A linear calibration equation was determined and used to predict the brain temperature in six new-born human infants to a precision of $\pm 0.4^{\circ} \mathrm{C}$. However, the reliability of the water chemical shift measured by ${ }^{1} \mathrm{H}$ MRS as a method of in vivo temperature determination has recently been questioned (Frenzel et al, 1996; Zuo et al, 1996). The main objection to the method is the relatively small change in the water chemical shift with temperature, approximately $0.01 \mathrm{ppm}^{\circ} \mathrm{C}^{-1}$ (Cady et al, 1995), which requires the magnetic field to remain highly stable during the study, a condition that is often difficult to achieve in clinical situations (Young et al, 1995). Frenzel et al (1996) studied the temperature dependence of ${ }^{1} \mathrm{H}$ MRS measurements of the proton shift in an intravenously injected lanthanide complex, relative to the water signal, in the livers of anaethetised rats. They observed a relative change of approximately $0.13 \mathrm{ppm}^{\circ} \mathrm{C}^{-1}$, more than an order of magnitude greater than that seen with the water proton shift, although this was at the expense of the associated invasiveness of using an external indicator compound administered by intravenous injection.

The spin-lattice relaxation time $\left(T_{1}\right)$ characterises the time taken for an excited spin system in an oscillating magnetic field to reach an equilibrium along the axis of the applied field. In NMR studies of biological tissue, $T_{1}$ is seen to vary with tissue type. The temperature-dependence of the spin-lattice relaxation time also varies with tissue type 
and is approximately linear in the temperature range of $20-50^{\circ} \mathrm{C}$ (Bertsch et al, 1998). Bertsch et al (1998) investigated the temperature-dependence of $T_{1}$ in various ex vivo tissue samples. In homogeneous samples of turkey breast tissue they obtained temperature predictions accurate to $\pm 2{ }^{\circ} \mathrm{C}$, relative to invasive temperature measurements, decreasing to an accuracy of $\pm 3.5^{\circ} \mathrm{C}$ in tissues containing a high percentage of fat. This was explained by the high temperature-dependence of the magnetic susceptibility in fatty tissues, which interferes with the calculation of temperature based on the measured changes in $T_{1}$ with temperature. As with the chemical shift method in magnetic resonance imaging (MRI), temperature monitoring via the $T_{1}$ relaxation time is essentially a difference technique.

The molecular water diffusion coefficient in tissue has also been investigated as a suitable parameter for MRI temperature measurements (Delannoy et al, 1991). Delannoy et al (1991) used diffusion imaging techniques to determine the temperature inside a gel phantom. They found the agreement between invasive measurements and non-invasive predictions of temperature to be better than $0.2^{\circ} \mathrm{C}$. They suggested that since the method determines only the change in temperature relative to some initial reference, absolute temperature measurement could be achieved by allowing the region of interest to be brought to an initial, uniform thermal equilibrium, e.g. by surrounding it with water at a known temperature. The temperature sensitivity of this method is reported to be twice that of the spin-lattice relaxation time method (Delannoy et al, 1991). The method can, however, suffer from poor temporal resolution and be highly sensitive to motion (Bertsch et al, 1998). Furthermore, the diffusion coefficient depends on tissue perfusion and compartment size, which can both change with temperature, confusing the relationship between the diffusion coefficient and the temperature (Bertsch et al, 1998; Frenzel et al, 1996).

\subsubsection{Ultrasound Thermometry}

The use of diagnostic ultrasound techniques have also been considered in an attempt to measure tissue temperature non-invasively. Seip and Ebbini (1995) related temperature changes to frequency changes in the spectrum of reflected ultrasound pulses. The underlying theory of the technique is based on the assumption that biological tissue consists of a semi-regular lattice of discrete scatterers, separated by some average distance $d$ over a certain region of interest. The frequency spectrum of back-reflected ultrasound pulses from tissue will therefore contain overtones of some 'fundamental' tissue frequency that will be related to $d$. This fundamental frequency $\left(f_{1}\right)$ is determined from the time taken for the ultrasound pulse to travel the minimum possible distance, (i.e. when it is reflected 
back by the first scatterer it encounters in the tissue lattice), and depends on both $d$ and the speed of sound in the tissue (Weng et al, 1992). Since $d$ and the speed of sound in tissue will change with temperature, and their temperature coefficients will vary between different tissue types, $f_{1}$ will also depend on tissue temperature (Seip and Ebbini, 1995). However, $f_{1}$ for most tissues does not lie within the frequency passband of typical diagnostic ultrasound transducers, therefore Seip and Ebbini (1995) investigated the temperature dependence of its visible harmonics. In an in vivo experiment they predicted the change in temperature of a dog's thigh due to ultrasonic heating by this method and found a reasonable agreement between these and invasive measurements of the temperature using a thermocouple. The standard deviation of the estimated results was however quite high at $0.5^{\circ} \mathrm{C}$, which the authors believed to be due to movement artifacts. Maass-Moreno et al (1996) used a similar technique to predict tissue temperature in turkey breast, relating temperature changes to the time shifts in the received ultrasound echoes. In between periods of heating with a high-intensity ultrasound beam, pulses were sent and received and the echo times recorded for the different temperatures. They noticed that the relationship between pulse-echo time and temperature related changes remained constant for a temperature increase of approximately $10^{\circ} \mathrm{C}$, but that as the temperature peaked the relationship appeared to change. The authors attributed this non-linearity to a result of irreversible tissue alterations at high temperatures (Maass-Moreno et al, 1996).

\subsection{Tissue Temperature Monitoring by NIRS}

The fundamental principal for all of the non-invasive techniques discussed in Section 4.2 is essentially the same: that some measurable signal detected from the brain, passively or otherwise, can be temperature-calibrated. For completeness, the technique of infrared thermography shall be mentioned, although it has not specifically been applied to measuring brain temperature. This is because the mid-infrared wavelengths employed, in the region of 5-15 $\mu \mathrm{m}$ (Togawa, 1985), are strongly absorbed by tissue water. Only radiation emitted from within a tissue depth of a few millimetres from the body's surface, i.e. the skin, will be detected. Hence, the temperature measurement, inferred by the assumption that the human body is a black-body radiator, pertains to the surface only and gives no information about deep body temperature (although diffusion of heat from the core will influence the surface measurement). In comparison to this and the other methods described in the previous section, the use of NIRS for monitoring brain tissue temperature 
offers some obvious advantages, as will be discussed in this section.

Firstly, NIR light can penetrate several centimetres into biological tissue (see Section 3.1), which ensures that the signal will contain contributions from deep brain tissue (see also Section 3.1.3). Furthermore, the equipment employed (Section 3.2) is relatively cheap, portable and simple to set up at the hospital bedside. Although the technique involves the active irradiation of tissue with NIR light, this is not considered to be invasive or damaging to tissue provided the safety standards for a maximum power input are adhered to (Cope, 1991). With regards to the temperature calibration, it is well-known that temperature is highly correlated to spectral changes in absorption by tissue chromophores, as will be discussed in Section 4.3.1. Hence, assuming that absorption can be separated from the scattering by appropriate modelling of the NIRS signal, one should be able to predict temperature by applying the previously determined temperature calibration of absorption. This provides NIRS with an advantage over methods, such as microwave radiometry, in which changes in other tissue properties (e.g. tissue dielectric properties) can interfere with the interpretation of the temperature-dependence of the signal. Since the penetration of the light will depend on its wavelength, it may also be possible to determine the temperature at different depths within the volume of tissue probed (see Chapter 8 for further discussion). Finally, the aim of this method is to determine absolute temperature in areas of suspected damage within the infant brain during hypothermic treatment. Provided the absolute absorption in the brain can be determined, absolute temperature quantitation should be possible. If the absolute quantitation of temperature is not possible, it may be that the determination of a temperature change in the brain will be sufficient provided that the initial temperature at depth can be estimated from e.g. skin surface measurements or heat-diffusion modelling. NIRS has the added advantage that it is currently employed in the assessment of cerebral oxygenation and haemodynamics, and could therefore be used to monitor temperature in conjunction with other cerebral parameters.

This section will discuss both the spectral changes in the tissue chromophore absorption coefficients and the physiological changes observed in tissue as a function of temperature, and hence the effects on whole-tissue absorption and scattering coefficients. In order to investigate its impact on tissue NIRS measurements, the effect of temperature on absorption and scattering will be studied separately. Absorption changes in the water spectrum will be discussed in some detail, since it is the main constituent of most soft tissues. 


\subsubsection{Changes in Absorption with Temperature}

\subsubsection{Water}

Absorption by water of electromagnetic energy in the infrared region is due to transitions between molecular vibrational and rotational levels. The vibrational motion of a water molecule can be described in terms of the three normal modes of vibration, the term 'normal' referring to the fact that the nuclei oscillate with the same frequency and phase (Eisenberg and Kauzmann, 1969). The three modes (see Figure 4.2) are known as the O-H symmetric stretch $\left(\nu_{1}\right)$, the $\mathrm{O}-\mathrm{H}$ antisymmetric stretch $\left(\nu_{2}\right)$ and the $\mathrm{H}-\mathrm{O}-\mathrm{H}$ bend $\left(\nu_{3}\right)$. The stretching modes are so called because the motions of the nuclei are very nearly parallel to the direction of the $\mathrm{O}-\mathrm{H}$ bonds, and similarly in the bending mode the vibrations occur in a direction almost perpendicular to the bonds (Eisenberg and Kauzmann, 1969).

In the vapour phase the $\nu_{1}, \nu_{2}$ and $\nu_{3}$ modes of vibration of a 'lone' water molecule are excited at frequencies in the mid-infrared corresponding to wavelengths of $2.73 \mu \mathrm{m}$, $6.27 \mu \mathrm{m}$ and $2.66 \mu \mathrm{m}$ respectively (Benedict et al, 1956). As water cools below $100^{\circ} \mathrm{C}$ and enters the liquid phase, intermolecular hydrogen bonding occurs. Hydrogen bonding affects the fundamental frequencies of the water molecules, and hence the absorption spectrum, in various ways. The $\mathrm{O}-\mathrm{H}$ stretches are facilitated by a hydrogen bond association with another molecule, which tends to reduce the $\nu_{1}$ and $\nu_{3}$ vibrational frequencies (Pimental and McClellan, 1960). In liquid water at $25^{\circ} \mathrm{C}$ a broad, irregularly shaped band with a principal maximum at about $2.87 \mu \mathrm{m}$ is observed (Bayly et al, 1963), which is associated
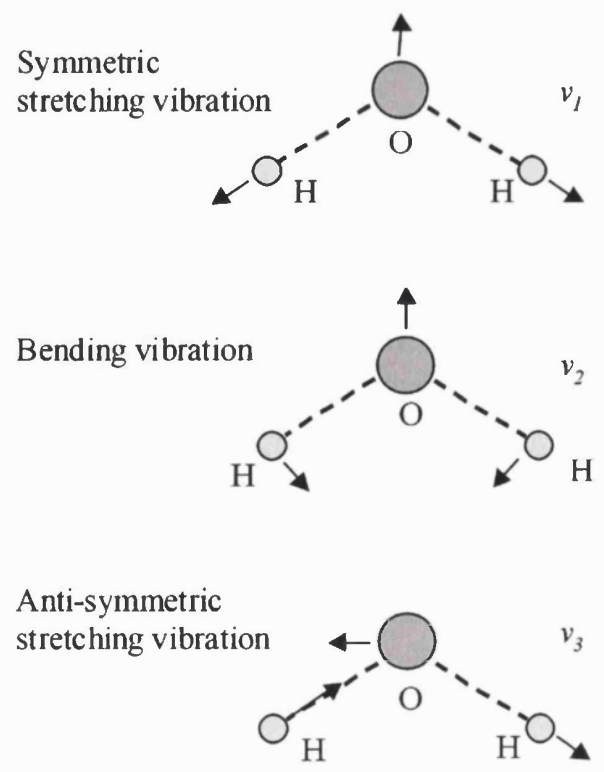

Figure 4.2: The three normal modes of vibration of a water molecule in the liquid phase. 
with the two stretching modes, reduced in frequency, and the first overtone of the $\mathrm{H}-\mathrm{O}-\mathrm{H}$ bending mode. The bending mode frequency increases with the degree of intermolecular hydrogen bonding and has an associated absorption band at around $6.08 \mu \mathrm{m}$ in water at $25^{\circ} \mathrm{C}$ (Bayly et al, 1963). Another notable effect of hydrogen bonding is the broadening of the fundamental absorption bands in liquid water relative to those in the vapour phase. This observation can largely be attributed to two effects:

i) due to the random structural order of liquid water the perturbation caused by hydrogen bonding can vary from molecule to molecule, producing a distribution of fundamental frequency shifts

ii) coupling of vibrational modes of similar frequencies between neighbouring molecules will cause a splitting of the shifted bands which may not be resolved, contributing to additional broadening (Eisenberg and Kauzmann, 1969).

The temperature-dependence of the fundamental vibrational bands of liquid water has been extensively studied (Eisenberg and Kauzmann, 1969). The majority of the work reviewed concentrates on the $\mathrm{O}-\mathrm{H}$ stretching bands in liquid water, since these have a greater temperature-dependence than the bending mode (Eisenberg and Kauzmann, 1969). Both Raman and infrared studies show that the integrated intensity of the combined stretching band decreases with increasing temperature (Walrafen, 1967; Libnau et al, 1994). The principal maximum of the band is seen to shift towards shorter wavelengths (i.e. higher frequencies), and the apparent width increases with temperature (Libnau et al, 1994). The interpretation of these temperature effects depends on the assumed model for the structure of water, for which many have been proposed. These models generally fall into two main classes:

i) continuum models

ii) mixture models.

The continuum model of liquid water implies an almost completely hydrogen-bonded molecular structure, in which a continuous distribution of intermolecular hydrogen bond strengths and angles exist (Wall and Hornig, 1965; Falk and Ford, 1966). The mixture model, on the other hand, suggests that a finite number of distinctly different structural species of water molecules exist in equilibrium (Buijs and Choppin, 1963; Walrafen, 1967). At least two different species are postulated: one involving 'free' or non-hydrogen-bonded 
molecules and another representing a bulky, hydrogen-bonded species (McCabe et al, 1970). The two schools of thought propose different explanations for the effects of temperature on the stretching bands $\nu_{1}$ and $\nu_{2}$ (Libnau et al, 1994). Walrafen (1967), a supporter of the mixture model, ascribed the structure of the Raman stretching band to the presence of various molecular species with different numbers of hydrogen bonds. He fitted a total of four Gaussian components to the band, measured over a range of temperatures. The intensity of the two higher frequency components increased with temperature; these he assigned to the vibrations of a non-hydrogen-bonded species. The two lower frequency components decreased in intensity with increasing temperature and were assigned to a hydrogen-bonded species, for which the $\nu_{1}$ and $\nu_{3}$ modes would, as previously discussed, be expected to vibrate at lower frequencies than those of a non-hydrogen-bonded species. Thus, Walrafen interpreted changes in the band shape with temperature in terms of a shift in the equilibrium between hydrogen-bonded and non-hydrogen-bonded species, due to breaking of intramolecular hydrogen bonds as the water temperature increased. From the continuum viewpoint, Schultz and Hornig (1961) argued that the temperature dependence of the stretching band can be explained in terms of Fermi resonance between the $\nu_{1}$ symmetric stretch mode and the first overtone of the bending mode, $2 \nu_{2}$. Fermi resonance, a 'borrowing' of intensity of one band from another, can occur when bands of a similar frequency overlap, which causes a shift in both band frequencies. The $2 \nu_{2}$ intensity alone would be much less than that of the fundamental $\nu_{1}$ band, but with the two in Fermi resonance with one another, the resulting band could be significantly shifted and broadened (Eisenberg and Kauzmann, 1969). Schultz and Hornig attributed the alterations in band shape to a change in the overlap, and hence Fermi resonance between, $\nu_{1}$ and $2 \nu_{2}$ caused by a continual shifting of the fundamental frequencies as a result of a change in intramolecular hydrogen bond strength with temperature.

In the NIR region, the water absorption bands are due to overtones and combinations of the fundamental $\nu_{1}, \nu_{2}$ and $\nu_{3}$ vibrations. The transitions that give rise to these bands are forbidden by quantum mechanical selection rules. In fact, due to the anharmonicity of the molecular vibrations, overtones of the fundamentals do occur, albeit at much lower probabilities (Pimental and McClellan, 1960). The assignment of bands in this region to particular overtones or combinations is, however, hindered by the possibility of many bands of similar frequencies overlapping one other. The bands of interest to the work in this thesis are those positioned at approximately 740,840 and $970 \mathrm{~nm}$. Bayly et al (1963) measured the absorption spectrum of water from $0.7-10 \mu \mathrm{m}$ and made assignments 
for the observed bands, comparing their results to those of other workers. They assigned the band at about $970 \mathrm{~nm}$ to the combination mode $2 \nu_{1}+\nu_{3}$, i.e. the first harmonic of the $\mathrm{O}-\mathrm{H}$ symmetric stretch in combination with the fundamental antisymmetric stretch, and the band at around $740 \mathrm{~nm}$ to the $3 \nu_{1}+\nu_{3}$ combination mode. However, there was disagreement over the assignment of the band at around $840 \mathrm{~nm}$, some workers suggesting it to be the combination of $2 \nu_{1}+\nu_{2}+\nu_{3}$, others assigning it to the $\nu_{2}+3 \nu_{3}$ combination mode. It must be noted that the temperature at which the water spectra were measured was not recorded for all the work listed, which could account for the disagreement between the workers in the assignment of the bands.

The temperature-dependence of the overtones and combination bands in the NIR has been studied by a number of workers, in particular by those wishing to investigate the hydrogen-bonded structure of water (Buijs and Choppin, 1963; McCabe et al, 1970; Libnau et al, 1994). Buijs and Choppin (1963) studied the bands in the region of 1.1 to $1.3 \mu \mathrm{m}$ as a function of temperature. They assigned the band structure in this region to the combination mode $\nu_{1}+\nu_{2}+\nu_{3}$. They explain its structure in terms of an overlapping of bands belonging to three different water species with the same vibrational mode assignment but forming different numbers of hydrogen bonds. The bands at 1.16, 1.20 and $1.25 \mu \mathrm{m}$ were attributed to a non-bonded species, a singly-bonded species and a doubly-hydrogen-bonded species respectively. Buijs and Choppin (1963) determined the absorption coefficients for the distinct bands at several temperatures and found the coefficient of the $1.16 \mu \mathrm{m}$ band to increase, i.e. as the number of non-hydrogen-bonded molecules increased, and the coefficient of the doubly-hydrogen-bonded species to decrease with temperature. The coefficient of the singly-bonded species increased slightly between 6 and $21^{\circ} \mathrm{C}$, presumably as the doubly-bonded species was converted into the singlybonded species, then decreased on further heating. Hornig (1964) strongly disputes Buijs' and Choppin's interpretation of the NIR band on the basis that the fundamental bands show no evidence of separate species (see Buijs and Choppin (1964) for reply). Libnau et al (1994) measured near- and mid-infrared spectra of liquid water at various temperatures. The NIR region between 1.1 and $1.25 \mu \mathrm{m}$ was assigned to both the $2 \nu_{1}+\nu_{2}$ and the $\nu_{2}+2 \nu_{3}$ combination bands. They found the integrated intensity of this multiple band to increase with temperature and the principal maximum of the band to shift to higher frequencies. Using a partial least-squares technique (see Section 5.3.6) they decomposed the spectra into two component spectra, one of which increased and the other which decreased in intensity with temperature. Kelly et al (1995) investigated the spectrum of water from 
850 to $1600 \mathrm{~nm}$ for temperatures between 17 and $45^{\circ} \mathrm{C}$. They found the band at 970 $\mathrm{nm}$ to increase in amplitude with increasing temperature, with a thermal isobestic point, i.e. a point at which the absorption is invariant with temperature, at about $1000 \mathrm{~nm}$. Hence the spectra have a positive temperature coefficient below the isobestic point, the greatest being at the band peak, and a negative coefficient above the isobestic point. This observation could be consistent with a change in band overlap and Fermi resonance with temperature, as proposed by supporters of the continuum model, although the presence of the isobestic point supports the theory that two absorbing species exist in equilibrium (Senior and Verrall, 1969). Del Bianco et al (2000) measured the spectrum of water in the range $850-1350 \mathrm{~nm}$ between 10 and $40^{\circ} \mathrm{C}$. They fitted the spectrum at $25^{\circ} \mathrm{C}$ with Gaussian components, finding a good fit over the range with six components. The spectra at all other temperatures were fitted using the same peak wavelength positions as for the fit to the spectrum at $25^{\circ} \mathrm{C}$ and allowing only the width and amplitude of the components to change with temperature. Of the six components, two accounted for the band at $970 \mathrm{~nm}$. The higher frequency component, at $964 \mathrm{~nm}$, showed an increase in amplitude with temperature, whilst the lower frequency component, at $992 \mathrm{~nm}$, decreased in amplitude with increasing temperature. The authors tentatively assigned the higher and lower frequency components to water species with a low and high number of hydrogen bonds respectively. Langford et al (2001) examined the temperature-dependence of the water absorption spectrum from $550-900 \mathrm{~nm}$ over the temperature range $15-60^{\circ} \mathrm{C}$. The absorption bands at approximately 740 and $840 \mathrm{~nm}$ were found to have comparable temperature coefficients, in the order of $0.8 \%{ }^{\circ} \mathrm{C}^{-1}$. These bands are of particular interest to the work in this thesis since, although their amplitudes are more than an order of magnitude smaller than for the band at $970 \mathrm{~nm}$ and hence the absolute signal changes with temperature are smaller, the penetration of NIR light into tissue is significantly greater at these shorter wavelengths.

\subsubsection{Haemoglobin}

As discussed in Section 2.1.2, haemoglobin is the complex molecule responsible for respiratory gas transport around the body. Haemoglobin absorption bands in the NIR are predominantly associated with charge-transfer transitions (Weissbluth, 1974). The NIR absorption spectra of haemoglobin in its oxygenated and deoxygenated forms (see Figure 2.2) are significantly different from one another. In deoxyhaemoglobin $(\mathrm{Hb})$, the charge-transfer transitions are between the iron centre and the surrounding porphyrin structure which, together, constitute the haem groups of the haemoglobin molecule. In 
oxyhaemoglobin $\left(\mathrm{HbO}_{2}\right)$, the broad spectral band centred at approximately $930 \mathrm{~nm}$ is associated with a charge-transfer transition between the iron and the oxygen molecule attached to it (Weissbluth, 1974).

The temperature-dependence of the haemoglobin NIR absorption spectra has been studied by various workers (San Bagio et al, 1977; Cordone et al, 1986; Sfareni et al, 1997). Sfareni et al (1997) measured the spectra of $\mathrm{Hb}$ and $\mathrm{HbO}_{2}$ in the region $700-1100 \mathrm{~nm}$ over the temperature range $20-40^{\circ} \mathrm{C}$. They expressed spectral changes with temperature in terms of the zeroth $\left(M_{0}\right)$ and first $\left(M_{1}\right)$ moments of the bands, which correspond to the overall intensity and the (weighted) peak wavelength respectively. For deoxyhaemoglobin $\mathrm{M}_{0}$ decreased by $0.15-0.18 \%{ }^{\circ} \mathrm{C}^{-1}$ and $\mathrm{M}_{1}$ altered by about $0.08 \mathrm{~nm}^{\circ} \mathrm{C}^{-1}$ (red-shifted for the band at $760 \mathrm{~nm}$ and blue-shifted for the band at $900 \mathrm{~nm}$ ), as temperature was increased from 20 to $40^{\circ} \mathrm{C}$. For oxyhaemoglobin $\mathrm{M}_{0}$ decreased by less than $0.05 \%{ }^{\circ} \mathrm{C}^{-1}$ and $\mathrm{M}_{1}$ red-shifted by about $0.15 \mathrm{~nm}^{\circ} \mathrm{C}^{-1}$ with increasing temperature. Cordone et al (1986) measured the $\mathrm{Hb}$ and $\mathrm{HbO}_{2}$ spectra between 20 and $300 \mathrm{~K}$ (i.e. -253 to $+27^{\circ} \mathrm{C}$ ). They observed similar spectral trends with temperature to those observed by Sfareni et al (1997). Cordone et al (1986) attributed the decrease in the integrated band intensities of both $\mathrm{Hb}$ and $\mathrm{HbO}_{2}$ with increasing temperature to a decrease in the overlap of the electron orbitals involved in the charge-transfer transitions.

\subsubsection{Whole Tissue}

The previous two sections have discussed changes in the absorption spectra of the major tissue chromophores caused by changes in intra- and inter-molecular forces with decreasing temperature. These spectroscopic changes are inherently related to both the chemical composition and physical structure of the chromophores themselves, and are observable in vitro in a laboratory setting. During NIRS measurements of living tissue, however, there will also be physiological changes within the region probed that are likely to affect the total absorption coefficient of the tissue, and hence the measured attenuation spectrum. A change in the temperature of the environment can activate a series of thermoregulatory responses in the body. For example, a reduction in ambient temperature will trigger cutaneous vasoconstriction, and thus a diversion of blood flow from surface to deep vessels in order to maintain the body's core temperature. Using NIRS techniques, Van Bel et al (1996) reported a decrease in cerebral blood volume with decreasing brain temperature in the neonate during hypothermic cardiopulmonary bypass, which would be observed as a reduction in the total haemoglobin concentration in the volume probed. Another NIRS 
study (Nomura et al, 1996) indicated an increase in mean cerebral oxygen saturation during cooling of the piglet brain. This can be explained by the decrease in metabolism with temperature and hence a reduction in the demand for oxygen, which leads to an increase in haemoglobin oxygen saturation because less oxygen is removed from the blood. Binzoni et al (1999) used NIRS methods to study oxygen consumption in the adult forearm as a function of temperature, and observed a drop in $\mathrm{O}_{2}$ consumption of approximately $55 \%$ as tissue temperature was decreased to approximately $26^{\circ} \mathrm{C}$. Furthermore, as temperature decreases the oxygen-haemoglobin dissociation curve shifts in such a way as to enhance oxygen binding to haemoglobin, thus augmenting the increase in saturation due to reduced metabolism. The absorption spectrum of cytochrome $c$ oxidase, the terminal enzyme in the mitochondrial electron transport chain (Section 2.1.4), depends on its redox state, which is also dependent on oxygen consumption (Springett et al, 2000). Hence, a reduced $\mathrm{O}_{2}$ consumption due to a decrease in tissue temperature will alter the wavelength-dependence of light absorption by cytochrome $c$ oxidase, although it should be noted that many electron transport chain reactions that influence the redox state of cytochrome $c$ oxidase will be temperature-dependent. Therefore it is important to consider both spectroscopic and physiological mechanisms for changes in the absorption of light by whole tissue.

Many workers have investigated the effect of increasing temperature on the absorption coefficient of tissue, e.g. to assess the dose given by laser-induced hyperthermia (Nilsson et al, 1998). Few studies could be found in which tissue optical properties had been measured at clinically hypothermic temperatures. The majority of the aforementioned hyperthermic studies were performed using excised tissue samples, in which the sample was heated from an ambient temperature of approximately $25^{\circ} \mathrm{C}$ and measurements made continuously during heating. In several of these studies workers found that the absorption coefficients of various types of tissue were not well correlated with, or were insensitive to, tissue temperature below about $45^{\circ} \mathrm{C}$, e.g. human skin (Laufer et al, 1998), bovine myocardium (Agah et al, 1996) and bovine liver (Jaywant et al, 1993). As mentioned in Section 3.3, the in vivo and ex vivo optical properties of tissue can vary considerably. In terms of its temperature response, an excised tissue sample will not behave in the same way as living tissue through which blood is continually flowing. Moreover, tissue water can be lost from an excised sample on heating causing shrinkage of the sample (Kelly et al, 1995), which could give significantly different results to the in vivo case.

The change in the optical properties of blood itself, however, can be studied in vitro and a comparison made with the in vivo properties in living tissues. Nilsson et al (1997) 
simulated the flow of human blood through a flow cell and measured its optical properties at $633 \mathrm{~nm}$, whilst slowly increasing the temperature from an ambient temperature of $30^{\circ} \mathrm{C}$. The absorption coefficient did not change until the temperature reached about $35^{\circ} \mathrm{C}$, then an increase in $\mu_{a}$ of approximately $35 \%$ between 35 and $45^{\circ} \mathrm{C}$ was observed, which was attributed to changes in absorption by haemoglobin. It should be noted, however, that other compounds in whole blood, e.g. other forms of haemoglobin, may also contribute to the change in $\mu_{a}$. Provided the maximum temperature reached did not exceed $45^{\circ} \mathrm{C}$, the increase in $\mu_{a}$ was seen to be reversible on cooling of the blood. In the same study, Nilsson et al also saw an irreversible change in $\mu_{a}$ when the blood was heated above $45^{\circ} \mathrm{C}$. They attributed this to the irreversible damage to the globin protein caused by heating, which results in the oxidation of the haem groups from their ferrous $\left(\mathrm{Fe}^{2+}\right)$ state to the ferric $\left(\mathrm{Fe}^{3+}\right)$ state. This oxidised form of haemoglobin is known as methaemoglobin (metHb), and has a characteristic absorption band in the region of $630 \mathrm{~nm}$ (Farahani et al, 1999).

Yeh et al (2001) performed in vivo NIRS measurements of the diffuse reflectance from the human adult forearm over a range of temperatures above and below core body temperature. In one experiment they cycled temperature between 22 and $38^{\circ} \mathrm{C}$, with a time period of about 6 minutes, for 90 minutes. The absorption coefficient at 590 and 950 $\mathrm{nm}$, determined by the use of MC modelling (see Section 2.3), increased by approximately $1 \%{ }^{\circ} \mathrm{C}^{-1}$ between 22 and $38^{\circ} \mathrm{C}$. (Changes in $\mu_{s}^{\prime}$ were also determined, but will be discussed in Section 4.3.2.) In a further experiment, temperature was swung between 25, 34 and $41^{\circ} \mathrm{C}$ and the absorption coefficient at $590 \mathrm{~nm}$ recorded. Once the temperature had reached $41^{\circ} \mathrm{C}$ they observed a continual upward drift in $\mu_{a}$ and only a slight decrease in its absolute value on reducing the arm temperature back to $25^{\circ} \mathrm{C}$. The authors attributed the changes in the absorption coefficient to changes in cutaneous blood content in the volume of interest. The source-detector separations used were relatively small (0.45$1.9 \mathrm{~mm}$ ), suggesting that the majority of the light would have propagated through the dermis, which is extremely well-vascularised and contains the capillary loops involved in thermoregulation. Hence, the assumption that changes in blood perfusion will be the dominant effect on $\mu_{a}$ is reasonable, particularly at $590 \mathrm{~nm}$ where absorption by water is negligible. No suggestions were made for why the change in $\mu_{a}$ was not immediately reversible above $40^{\circ} \mathrm{C}$. It is possible, though, that as the temperature is increased and blood flows into the superficial vessels, the vasoconstriction that occurs on a reduction in skin temperature will leave a certain amount of blood in the surface capillaries, preventing an immediate return to baseline levels of blood flow or volume in the region probed. 


\subsubsection{Changes in Scattering with Temperature: Whole tissue}

The scattering of light by tissue, in addition to the total absorption, may also be altered by a decrease in tissue temperature. The implications are the same as those detailed in Section 4.3.1.3 for absorption: a change in the scattering coefficient will affect the distribution of the light within the tissue and hence the measured attenuation spectrum. If information about the tissue temperature is to be extracted from the NIR spectrum, the effect of temperature upon the scattering coefficient must be understood.

The relevance of using excised samples to study the effect of temperature on in vivo tissue absorption coefficients was questioned in Section 4.3.1.3 since both blood flow and oxygen consumption, changes in which are likely to the dominate changes in absorption, are not present in ex vivo samples. The average scattering coefficient of tissue depends on factors such as the relative refractive index between the scattering objects and their surrounding media, the size and shape of the objects and their number densities (see Section 2.2.2). Therefore, changes in the magnitude and wavelength-dependence of the scattering coefficient with temperature are more likely to be governed by morphological changes than functional ones such as oxygenation or blood perfusion (although the latter may also affect scattering, as will be discussed shortly). Disregarding any post-mortem effects that may alter the absolute value of $\mu_{s}^{\prime}$ (see Section 3.3), it may be possible that similar trends in $\mu_{s}^{\prime}$ with temperature will be observed in ex vivo samples in comparison to in vivo studies.

Red blood cells have been identified as efficient scatterers of light (Sections 2.2.2 and 3.3), hence any change in their scattering properties as a function of temperature may affect the average scattering coefficient of well-perfused in vivo tissues. In the study mentioned in Section 4.3.1.3, Nilsson et al (1997) observed a reversible increase in the transport scattering coefficient of whole blood at $633 \mathrm{~nm}$ of approximately $12 \%$ between 35 and $45^{\circ} \mathrm{C}$. When the blood was heated above $45^{\circ} \mathrm{C}, \mu_{s}^{\prime}$ did not return to baseline on cooling but remained positively offset, i.e. the change was irreversible. The red blood cells were studied under a microscope and were seen to have transformed from the typical biconcave disc shape to spheres within a small temperature interval above $45^{\circ} \mathrm{C}$. This shape transformation was reported to be due to the effect of heating on structural proteins within the cells and was used to explain the changes in the anisotropy factor $g$ and the scattering coefficient $\mu_{s}$ (and hence $\mu_{s}^{\prime}$ ) on reaching the critical temperature. In addition to a change in the scattering properties of blood, a change in blood volume or flow with temperature may also affect the in vivo scattering coefficient of tissue, essentially by changing the 
concentration of scatterers present in the region probed. Depending on the tissue types present in the region of interest, however, this is likely to be a relatively small effect. For example, assuming that blood typically occupies $2-3 \%$ of brain volume in the new-born infant (Meek et al, 1999), a reduction in blood volume of $10-20 \%$ due to a $5^{\circ} \mathrm{C}$ decrease in brain temperature (Van Bel et al, 1996), would only reduce the percentage of blood in the brain by $0.2-0.6 \%$.

As mentioned in Section 4.3.1.3, much of the work on the temperature-dependence of tissue optical properties has been performed on excised tissue samples as a function of increasing temperature. At approximately $45^{\circ} \mathrm{C}$, irreversible thermal denaturation of proteins will occur, a complex process in which the protein structure is modified into a more random arrangement (Essenpreis, 1992). The effect of thermal denaturation on light scattering can be seen when cooking an egg, where upon heating the egg 'white' turns from clear to opaque as the protein structure is irreversibly altered. Denaturation can also be accompanied by thermal coagulation, the formation of the altered proteins into dense aggregates. These processes have been seen to occur in studies of ex vivo tissue samples, as confirmed by histological findings, with a concomitant change in the scattering coefficient (Jaywant et al, 1993). This supports the argument that the structural changes in living tissue as a function of temperature will also be seen in excised samples, making the comparison between in vivo and ex vivo studies of the scattering coefficient more plausible than for the absorption coefficient. Below a temperature of approximately $43^{\circ} \mathrm{C}$ thermal damage, defined as any structural changes in tissue due to heating above $37^{\circ} \mathrm{C}$ (Essenpreis, 1992), can be reversible provided it is not prolonged. Hence, one could conclude that structural changes can also occur in tissues that are cooled below normal body temperature, and that these changes will also be reversible.

Laufer et al (1998) studied the effect of temperature on the optical properties of excised samples of human skin. Dermis and subdermis were measured separately in the temperature range $25-40^{\circ} \mathrm{C}$. No significant change in the wavelength-dependence of the transport scattering coefficient was observed, therefore an average temperature coefficient was expressed, which was positive for the dermis and negative for the subdermis. The positive temperature coefficient for $\mu_{s}^{\prime}$ of the dermis of $0.47 \pm 0.05 \%{ }^{\circ} \mathrm{C}^{-1}$ was tentatively attributed to reduced hydration, and hence size, of the protein-rich collagen fibres that are deemed to be the dominant scatterers in the dermis. The negative temperature coefficient for $\mu_{s}^{\prime}$ of the subdermis of $-0.14 \pm 0.03 \%{ }^{\circ} \mathrm{C}^{-1}$ was believed to be due to the temperature-dependent behaviour of the lipids in the cellular membranes and adipose 
tissue abundant in the subdermis. As temperature increases the lipids will undergo a transition from an ordered gel phase to a more randomly ordered liquid phase (Mantsch et al, 1985). Chong and Colbow (1976) studied the change in the light intensity scattered from various lipid dispersions as a function of temperature. They showed that a large part $(\approx 7 / 8)$ of the reversible decrease in scattered light intensity with increasing temperature in the range $20-50^{\circ} \mathrm{C}$ could be accounted for by a reduction in the refractive index of the lipids at the phase transition. In different dispersions, however, Chong and Colbow observed irreversible changes in scattered light intensity, which they attributed to the fusion of smaller lipid vesicles during the gel/liquid phase transition. One could argue that below the thermotropic phase transition temperature of approximately $41^{\circ} \mathrm{C}$ (Chong and Colbow, 1976), there should be no irreversible effect on the scattering by lipid membranes. However, Mantsch et al (1987) point out that in live cells, certain fatty acid chains present in the membranes can affect the phase transition temperatures of phospholipids, complicating the modelling of light scattering by live cells as a function of temperature.

On the basis of their observations, Laufer et al (1998) comment that for tissues in which the protein and lipid contents are comparable, the average scattering coefficient may show little change with temperature due to a cancellation of the opposing effects of protein and lipid. Jaywant et al (1993) studied the optical properties of calf brain with increasing temperature in the range $30-80^{\circ} \mathrm{C}$. They found that the transport scattering coefficient at $633 \mathrm{~nm}$ of white matter did not change significantly over the whole temperature range, and for grey matter $\mu_{s}^{\prime}$ increased only beyond $50^{\circ} \mathrm{C}$. Although no values could be found for bovine brain, the protein-to-lipid ratio will be higher in grey matter than it is in white matter, as is the case for human brain (White et al, 1991). Hence, the observed increase in $\mu_{s}^{\prime}$ for grey matter could be due to the increasingly larger effect, with respect to that of decreasing lipid refractive index, of protein denaturation above $50^{\circ} \mathrm{C}$.

Jaywant et al (1993) also studied the optical properties of bovine liver and muscle tissue with increasing temperature. For liver the transport scattering coefficient increased steadily with temperature beyond $40-43^{\circ} \mathrm{C}$, the change at $633 \mathrm{~nm}$ being larger than that at $810 \mathrm{~nm}$. Histological findings at high temperatures showed that the cellular components in the liver tissue had denatured and coagulated. The authors attributed the steady increase in $\mu_{s}^{\prime}$ with temperature to the continual denaturation and aggregation of globulin proteins in the liver cells at various temperatures. In the muscle tissue the transport scattering coefficient increased immediately upon heating, with an increase of about $17 \%$ between 30 and $40^{\circ} \mathrm{C}$, the changes again being greater at shorter wavelengths. Above 
about $45^{\circ} \mathrm{C} \mu_{s}^{\prime}$ increased at a greater rate, the two different slopes being attributed to the denaturation of first actin proteins then myosin proteins in the muscle fibres with increasing temperature. The histological analysis showed that the myofibril structure had been partially preserved at $80^{\circ} \mathrm{C}$, which explained the fact that the absolute change in $\mu_{s}^{\prime}$ of the muscle was approximately half that of the liver tissue, in which the scattering centres had become increasingly random in arrangement with increasing temperature. An important point to remember is that the change in $\mu_{s}^{\prime}$ reported by these studies could be due to a change in either $\mu_{s}$, the scattering coefficient, or $g$, the anisotropy factor, or both. It is possible that denaturation and coagulation of protein-rich tissues will cause an increase in $\mu_{s}$ due to an increased number density of the average scattering centres, and a decrease in $g$ due to a reduction in their size. Both these effects will act to increase $\mu_{s}^{\prime}$ with increasing temperature. Jaywant et al (1993) report a smaller change in the total attenuation coefficient $\mu_{t}$ than for $\mu_{s}^{\prime}$, which indicates that the anisotropy term $(1-g)$ increases more rapidly with temperature than $\mu_{s}$.

Only one in vivo study could be found in which the optical properties of tissue were measured as a function of temperature. In the study mentioned previously in Section 4.3.1.3, Yeh et al (2001) determined $\mu_{a}$ and $\mu_{s}^{\prime}$ in the adult human forearm with increasing temperature. The changes in $\mu_{s}^{\prime}$ with temperature were always reversible during heating and cooling between 22 and $38^{\circ} \mathrm{C}$, and when the temperature was switched between 25,34 and $41^{\circ} \mathrm{C}$. In the range $22-38^{\circ} \mathrm{C}$ the temperature coefficient for $\mu_{s}^{\prime}$ of the adult forearm was approximately 0.46 and $0.34 \%{ }^{\circ} \mathrm{C}^{-1}$ at 590 and $950 \mathrm{~nm}$ respectively. These are close in value to the wavelength-independent temperature coefficient of $0.47 \%{ }^{\circ} \mathrm{C}^{-1}$ found by Laufer et al (1998) for their ex vivo study of the human dermis. However, Yeh et al (2001) argue a different explanation to Laufer et al (1998) for the observed changes in $\mu_{s}^{\prime}$ of the dermis. Yeh et al (2001) used Mie theory to simulate the effect on $\mu_{s}^{\prime}$ of a change in the refractive index of the interstitial (or intercellular) fluid (ISF) with temperature. They simulated the change in the refractive index of the ISF as a function of temperature by using typical values for its protein content. The refractive indices of the scatterers were assumed to remain the same as temperature changed, and a range of values typical of scattering centres within the dermis, such as cell membranes and collagen fibres, were used. The simulations predicted changes in $\mu_{s}^{\prime}$ of between 0.25 and $0.85 \%{ }^{\circ} \mathrm{C}^{-1}$ at 590 $\mathrm{nm}$. The authors thus concluded that the changes they had observed in $\mu_{s}^{\prime}$ of the human forearm could be attributed to a change in the refractive index mismatch between the ISF and the scattering centres within the tissue as temperature was altered. 


\subsubsection{Effect of Temperature on In Vivo NIRS Measurements}

The optical properties of various tissues as a function of temperature were considered in Sections 4.3.1.3 and 4.3.2. An important point to consider now is the effect of temperature on in vivo NIRS measurements of the head and limbs. The total absorption and scattering coefficients measured will obviously be influenced by and contain contributions from the each of the tissue layers in the region probed. In attempting to determine the relative contributions of each tissue type to the temperature-dependence of the total optical properties in the head and limbs, the important factors are the dominant temperature effects in each tissue layer and the fraction of the light that propagates through each of them.

From the findings of Section 4.3.1.3, it appears that the dominant effects of temperature on absorption by living tissue will be:

- changes in the concentrations of $\mathrm{Hb}$ and $\mathrm{HbO}_{2}$ due to changes in perfusion and metabolism

- wavelength-dependent changes in the absorption spectra of water and haemoglobin.

The skin, skull, brain and muscle tissue are all vascularised, therefore a reduction in tissue perfusion with decreasing temperature could contribute to absorption changes in each tissue. As the total absorption coefficient changes, e.g. due to decreased perfusion in some or all of the layers, the penetration of the light will change, which could also alter the relative concentrations of the chromophores within the volume probed. The possible effect of temperature on absorption by cytochrome $c$ oxidase was mentioned in Section 4.3.1.3. However, given the relatively small contribution of cytochrome $c$ oxidase to the total absorption in tissue (Section 2.1.4), its contribution to a change in absorption with temperature is also likely to be small.

Possible causes for the temperature-dependence of the scattering coefficient in tissue, identified in Section 4.3.1.3, include:

- structural changes in proteins, e.g. collagen in the dermis or actin in muscle tissue

- changes in the relative refractive index between e.g. phospholipid cell membranes or triglyceride fat cells and interstitial fluid.

Skin contains both protein and lipids, the former being present in the dermis and the latter being the main constituent of the subdermis. Brain tissue also contains both components, 
the ratio of lipid to protein increasing with development of the foetal and infant brain (White et al, 1991). The neonatal skull contains a relatively high proportion of collagenous proteins compared to the adult and a low degree of mineralisation. The dense cortical bone of the skull contains no lipids, but the spongy bone between the dense plates contains the fatty marrow responsible for red blood cell production. As mentioned in Section 4.3.2, the temperature coefficient of the scattering coefficient in some lipid-rich tissues is positive, i.e. in the same direction as the temperature change, whilst in protein-rich tissues it appears to be negative. However, it is not apparent which of these effects would be dominant during in vivo spectroscopic measurements, since the relative contributions of lipid and protein in the volume probed are unknown and will be highly age-dependent.

The overall effect on in vivo NIRS measurements of changes in tissue temperature, e.g. during hypothermic treatment, will depend on the relative pathlengths of the light through the each tissue layer. An important factor to consider is the change in the NIR penetration depth with optical properties. If $\mu_{a}$ increases with temperature, as was shown by Yeh et al (2001), then the penetration depth will decrease, as predicted by diffusion theory (Section 3.1.3). Hence, the volume of tissue sampled may vary with temperature, which, in addition to the temperature-dependence of the optical properties of each tissue type, will induce a further change in the average optical coefficients with temperature. 


\section{Chapter 5}

\section{Characterisation of the NIR Water Absorption Spectrum}

As outlined in Chapter 4, the basis of this work is to exploit the temperature-dependence of the NIR water absorption spectrum in order to determine tissue temperature from NIRS measurements. This chapter describes the primary goal: to characterise the water spectrum as a function of temperature in the clinical range. This initial aim was subdivided into specific tasks, the implementation of which is discussed in the following sections.

Section 5.1 describes the experimental design required to accurately measure the water absorption spectrum over the temperature range of interest, and displays and discusses the results obtained. Since some of the water in body tissues is bound to other biomolecules and ions, as discussed in Section 2.1.1, experiments were performed to investigate the effects of water binding on the temperature response of the water spectrum, the results of which are described in Section 5.2. Finally, Section 5.3 will discuss the development of methods used to calibrate the temperature-dependence of the water spectrum, hence providing a means of predicting temperature from measurements of water absorption. 


\subsection{Measuring the Temperature Response of the Water Ab- sorption Spectrum}

In Section 4.3.1.1 the temperature-dependence of absorption of electromagnetic radiation by water was discussed in detail. Changes in intermolecular hydrogen-bonding was cited as the cause of the shifts in the mid-infrared fundamental vibrational bands with temperature. As a consequence, the NIR bands (combinations and overtones of the fundamentals) are also sensitive to temperature. For this temperature-dependence to be employed in predicting tissue temperature, it must first be accurately determined. This section describes the development of an experimental methodology for the measurement of the NIR water absorption spectrum and its temperature response in the range $28-44^{\circ} \mathrm{C}$.

\subsubsection{Experimental Design}

The basic equipment used for all spectroscopic measurements described in this project was covered previously in Section 3.2 (shown schematically in Figure 3.2). Therefore, this section will discuss only the design of the specific set-up used for measuring the temperature-dependence of the water spectrum.

Prior to deciding upon an experimental method, the following design requirements for the set-up were identified:

- to avoid temperature-independent changes in water absorption affecting the measurements, the sample must be positioned such that the concentration of water molecules in the light path does not change with water expansion (or contraction) due to temperature changes

- since the measured absorption changes are small, the coupling of the emergent light into the detecting fibre must be efficient and relatively insensitive to small spatial or angular changes in light distribution

- the experimental set-up as a whole must be placed within a temperature-controlled environment with a uniform temperature distribution.

Certain requirements of the experimental method resulted in the development of two distinct methodologies, which shall be discussed in the following sections. The final design for the set-up, however, was common to both methods and is shown in Figure 5.1. The entire set-up was mounted on an optical board of approximate dimensions 30 by $45 \mathrm{~cm}$. 


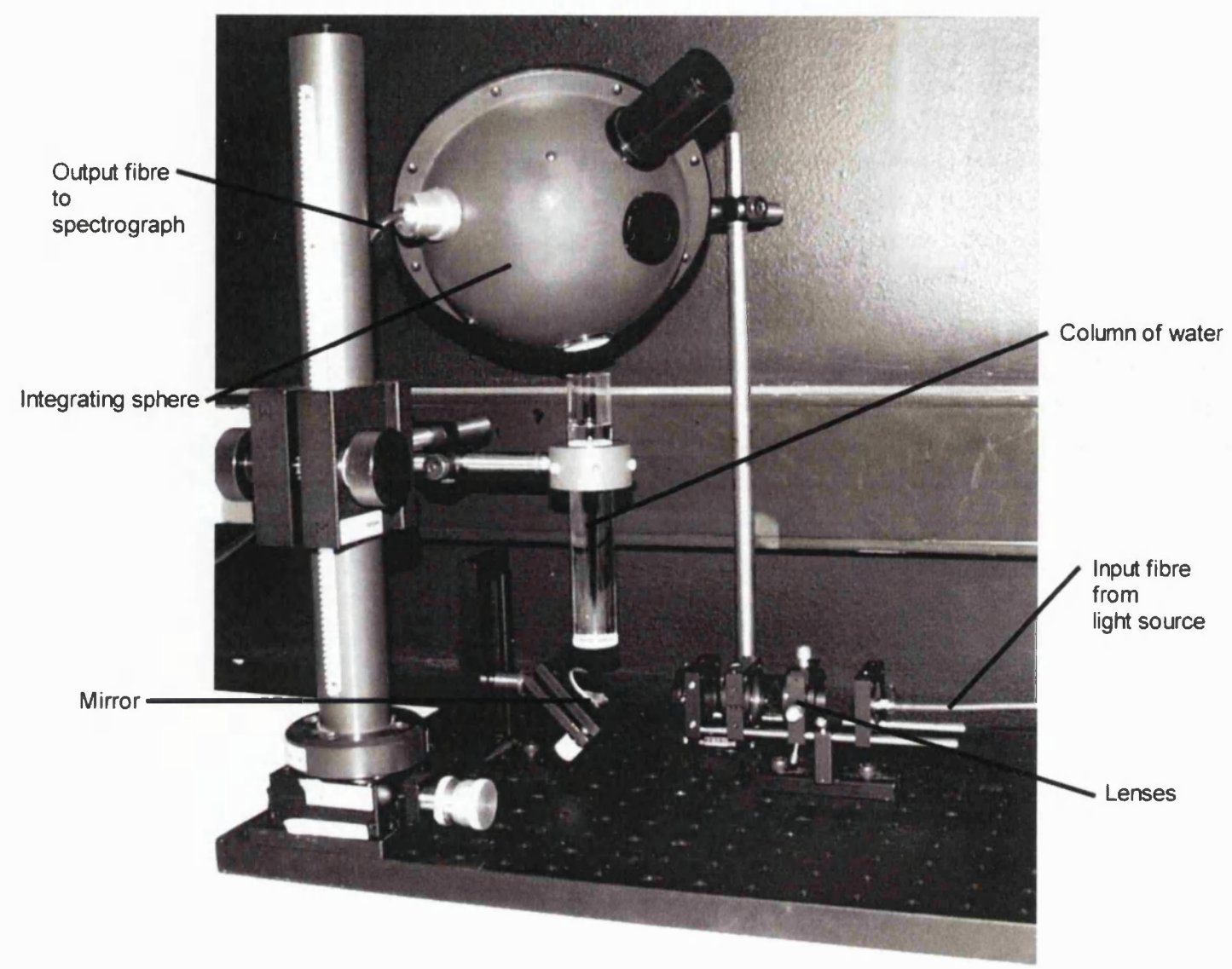

Figure 5.1: Experimental set-up for the measurement of the NIR water absorption spectrum and its temperature response.

As indicated in Figure 5.1, the light is coupled from the source to the set-up via a fibre optic of diameter $200 \mu \mathrm{m}$. The beam is then collimated and expanded to a diameter of approximately $5 \mathrm{~mm}$ by a system of lenses and an adjustable aperture. A mirror angled at $45^{\circ}$ to the board directs the beam vertically upwards through a glass tube containing the water sample. The tube is $2.5 \mathrm{~cm}$ in diameter and $15 \mathrm{~cm}$ in height, with a borosilicate glass window (Melles Griot) adhered to the base. This vertical positioning of the tube satisfies the first design requirement listed above by ensuring that the same number of water molecules (on average) remain in the path of the light during either water expansion or contraction due to changes in temperature (see Section 5.1.2.2 for further discussion). The light emerging from the sample is collected in an integrating sphere (RT060-SF, LabSphere) positioned with its open port-hole directly above the tube. The light is then coupled to the spectrograph-CCD system described in Section 3.2 via another fibre. The walls of the integrating sphere are designed to be highly reflective in order to diffuse the light uniformly around the interior of the sphere. Although much of the input light is attenuated due to multiple reflections, the output produced is highly uniform and, as 
stipulated by the second requirement listed above, is insensitive to small spatial or angular changes in the input, e.g. due to changes in the refractive index of water with temperature.

To satisfy the final design requirement the whole set-up displayed in Figure 5.1 was placed inside a neonatal incubator, within which the temperature could easily be controlled and a uniform temperature distribution reached. A safety feature of the incubator, intended during normal operation to inhibit the heating element once the internal temperature exceeds $39^{\circ} \mathrm{C}$, had previously been over-ridden (Cope, 1991). To prevent the detection of ambient light the incubator was made light-tight by covering it with thick black cloth, and all measurements were made with the room lights off.

\subsubsection{Experimental Methods and Results}

The absorption spectrum of distilled water in the region $650-1050 \mathrm{~nm}$ was measured over the temperature range $28-44^{\circ} \mathrm{C}$, using the set-up described in Section 5.1.1. To be successful the experimental method employed had to fulfil the following criteria:

- water temperature must be accurately determined and monitored throughout the experiment without affecting the spectroscopic measurements

- to determine the exact water height, and hence absorption, water added to the system must be done so accurately and without disturbing the optical set-up or affecting the measurements

- to obtain absolute measurements of water absorption the refractive index mismatches at the glass-air/glass-water interfaces must be accounted for

- water loss due to evaporation during the experiment must be prevented or minimised.

Common to the two methodologies to be described shortly was the manner in which the water temperature was measured. This was done using two calibrated temperature transducers (AD592, Maxim) mounted on the outside of the glass tube and held in place by a polystyrene insulating jacket fastened around the tube (not shown in Figure 5.1). A small amount of heat transfer compound provided good thermal contact between the tube and the sensors. The accuracy of the sensors was quoted to be $\pm 0.1^{\circ} \mathrm{C}$ after further calibration, which was performed using a precision mercury thermometer. A monitoring circuit was designed which converted the $1 \mu \mathrm{A}{ }^{\circ} \mathrm{C}^{-1}$ current output to a voltage (with a suitable gain), which was then input into the channels of a 12-bit analogue-to-digital (AD) 
computer card. This enabled the temperature to be sampled and recorded simultaneously with the spectral data by the acquisition software. The two sensors were placed at top and bottom ends of the tube in order to determine if any temperature gradient existed within the column of water. For the experiments in which the water height was at its maximum (i.e. the measurements of the smaller absorption bands, as discussed in Section 5.1.2.1), the temperature difference between the sensors positioned at the two extremes was always in the range $\pm 0.2^{\circ} \mathrm{C}$, indicating no significant temperature gradient. To determine how accurately the sensors mounted on the outside of the tube measured water temperature within the tube, a comparison was done with a mercury thermometer positioned at two different heights inside the tube of water. With the thermometer held at the bottom of the tube the sensor at the corresponding position on the outside of the tube was within $0.3^{\circ} \mathrm{C}$ of the mercury reading at a stable temperature of about $41^{\circ} \mathrm{C}$, this error approaching $0.2^{\circ} \mathrm{C}$ when the temperature cooled to $27^{\circ} \mathrm{C}$. Slightly smaller errors were seen for the second sensor when the procedure was repeated with the mercury thermometer positioned at the top of the tube.

Water was added to the tube through a thin plastic catheter using an electronic syringe driver with a reported accuracy of $\pm 3 \%$ (Medfusion 2010, Medex). This enabled water to be introduced without adjusting the set-up, the significance of which will become clearer in the subsequent sections, and to a known height (using a digital micrometer the inner diameter of the tube was determined to be $20.6 \pm 0.1 \mathrm{~mm}$ ).

Attempting to fulfil the final two criteria listed above led to the use of two different methods for measuring the temperature-dependence of the water spectrum, as shall be described here. To help explain the need for the second method, the results of the first method are discussed directly after its description.

\subsubsection{Method I}

Using the first method the three NIR absorption bands, centred at approximately 740 , 840 and $970 \mathrm{~nm}$, were measured separately, in order to study their temperature responses in detail. The actual wavelength ranges covered were $650-805 \mathrm{~nm}, 765-920 \mathrm{~nm}$ and 900 $1050 \mathrm{~nm}$. Using the 600 grooves $\mathrm{mm}^{-1}$ dispersion grating and a spectrograph slit width of $100 \mu \mathrm{m}$ gave a spectral resolution of $0.62 \mathrm{~nm}$ (see Section 3.2). During heating of the incubator, the rate of change in the temperature of the water sample proved difficult to control, being either too fast to provide sufficient temperature resolution, or too variable due to the feedback mechanism of the incubator's heating element. Spectral measurements 
were therefore made during cooling of the incubator, from a maximum temperature of approximately $44^{\circ} \mathrm{C}$ to a minimum of around $30^{\circ} \mathrm{C}$.

In order to obtain absolute measurements of water absorption using this set-up, (the third criterion listed in Section 5.1.2), a reference measurement through $1 \mathrm{~cm}$ of water was performed for each range. The absolute measurements were then made after adding a further volume of water, the optical path through the water (for the purpose of absorption calculation) being equivalent to this additional height. This avoids the problem of differences in light losses due to reflection at the glass-air interface, if no water is present for the reference measurement, and at the glass-water interface when the water is then added for the absolute measurement (see Section 5.1.2.2 for further clarification of this point).

Once the reference volume of water had been added, (using the syringe driver described in Section 5.1.2), the optical alignment of the set-up was adjusted by altering the angle of the mirror and tube to ensure that the light beam was passing straight through the centre of the tube and into the porthole of the integrating sphere above. Prior to heating the reference volume to the desired starting temperature, a thin layer of silicone oil was added to prevent water evaporation during the experiment, the fourth requirement of the experimental method discussed in Section 5.1.2. The incubator was then closed and heated until a stable temperature of around $44^{\circ} \mathrm{C}$ was recorded by the temperature sensor at the bottom of the tube, in thermal equilibrium with the reference volume of water, and a reference measurement was made. The spectral acquisition times were chosen such that, on addition of the further volume for absolute measurements, the signal recorded at the maximum temperature would be around 150,000 electron counts. This allows scope for the expected increase in intensity with decreasing temperature, without saturation of the CCD (see Section 3.2). Since the amount of absorption at the three different ranges varies considerably both the acquisition times and absolute heights were varied accordingly. To improve the SNR (Section 3.2) the spectra were averaged together by the acquisition software such that one spectrum was stored every 2 seconds, i.e. the averaging factor for a given range was equal to the number of acquisition times occurring in 2 seconds. Table 5.1 shows how these parameters were varied for each of the three ranges. The differences in the absolute heights added reflects the differences in the magnitude of the absorption coefficient in the three ranges (see Figures 5.3-5.5 in Section 5.1.2.2). It should be noted that the acquisition software averages together the logarithm (base 10) of the spectral intensities (calculated internally), not the intensities themselves, since the attenuation 


\begin{tabular}{llll}
\hline $\begin{array}{l}\text { Measured } \\
\text { band }(\mathrm{nm})\end{array}$ & $\begin{array}{l}\text { Absolute } \\
\text { height }(\mathrm{cm})\end{array}$ & $\begin{array}{l}\text { Acquisition } \\
\text { time }(\mathrm{ms})\end{array}$ & $\begin{array}{l}\text { Averaging } \\
\text { factor }\end{array}$ \\
\hline 740 & 12 & 100 & 20 \\
840 & 12 & 200 & 10 \\
970 & 1 & 500 & 4 \\
\hline
\end{tabular}

Table 5.1: Experimental and acquisition parameters used during the measurement of water absorption by Method I

averaged over $n$ spectra would be given by

$$
A_{a v g}=\log _{10} I_{r}-\frac{1}{n} \sum_{i=1}^{n} \log _{10} I_{i}
$$

where $I_{r}$ and $I_{i}$ are the reference intensity and the $i$ th sample intensity measurement respectively. Clearly, the average of the logarithm of the intensities is not equal to the logarithm of the averaged intensities

$$
\frac{1}{n} \sum_{i=1}^{n} \log _{10} I_{i} \neq \frac{1}{n} \log _{10} \sum_{i=1}^{n} I_{i}
$$

and therefore it must be the logarithm of each intensity that is averaged in order to subsequently calculate average attenuation and hence absorption.

After the reference measurement through $1 \mathrm{~cm}$ of water was made, the additional volume was added, again using the syringe driver. Adding the water via the plastic catheter ensured that the set-up did not have to be moved in any way, which avoided disturbing the optical alignment. Moreover, since the catheter was small enough to feed through a delivery hole in the incubator wall, water could be delivered into the glass tube without opening the incubator and affecting the temperature inside. As will be shown in the following results section, it was important for the accurate determination of the water absorption coefficient that, before absolute measurements were begun, the temperature of the additional water reached the same as that at which the reference measurement was made. Therefore, absolute measurements began once the temperature recorded by the sensors was stable to within $\pm 0.2^{\circ} \mathrm{C}$ of the reference temperature, allowing about 2 minutes of acquisition before the incubator temperature was reduced to $30^{\circ} \mathrm{C}$. The number of spectra collected was set to 8000 , with a total measurement time of approximately 4.5 hours. 


\subsubsection{Analysis and Results I}

For each of the three ranges measured using Method I the spectral data was exported from the acquisition software and imported into MATLAB ${ }^{\circledR}$ (The MathWorks), where a userdefined program was written to calculate $\mu_{a \mathrm{~T}}$, the water absorption coefficient spectrum as a function of temperature. A discussion of the calculation of $\mu_{a \mathrm{~T}}$ from measurements obtained using Method I has been published elsewhere (Hollis et al, 2001). However, a more thorough analysis of this experiment showed the calculation to be erroneous due to a misconception regarding the effects of temperature under this measurement scheme. Although the quantitative effects of this are extremely small $(<0.2 \%)$, the issue is redressed here with a more rigorous analysis of the situation.

A simple but important point to reiterate is the fact that, due to the vertical alignment of the glass tube, it was not necessary to correct calculations of the absorption coefficient for changes due to water expansion with temperature. This is because changes in water concentration and height with temperature cancel one another out. If, for example, an initial volume $V_{1}$ is added to the tube at $40^{\circ} \mathrm{C}$ such that a height of water $h_{1 \mathrm{~T}_{40}}$ is reached, the attenuation measured at that temperature can be expressed as

$$
A_{\mathrm{T}_{40}}=\varepsilon_{\mathrm{T}_{40}} c_{\mathrm{T}_{40}} h_{1 \mathrm{~T}_{40}}
$$

where $\varepsilon_{\mathrm{T}_{40}}$ is the specific absorption coefficient (defined in Section 2.1) at $40^{\circ} \mathrm{C}$ and $c_{\mathrm{T}_{40}}$ is the concentration of water molecules at $40^{\circ} \mathrm{C}$. The absorption coefficient at $40^{\circ} \mathrm{C}$ is calculated by dividing the attenuation by the height at that temperature

$$
\begin{aligned}
\mu_{a \mathrm{~T}_{40}} & =\frac{A_{\mathrm{T}_{40}}}{h_{1 \mathrm{~T}_{40}}} \\
& =\varepsilon_{\mathrm{T}_{40}} c_{\mathrm{T}_{40}}
\end{aligned}
$$

If the water in the tube is then cooled to $30^{\circ} \mathrm{C}$ such that its height becomes $h_{1} \mathrm{~T}_{30}$ and the concentration $c_{T_{30}}$, then the attenuation measured at this temperature is now

$$
A_{\mathrm{T}_{30}}=\varepsilon_{\mathrm{T}_{30}} c_{\mathrm{T}_{30}} h_{1 \mathrm{~T}_{30}}
$$

and the absorption coefficient at $30^{\circ} \mathrm{C}$ would be given by

$$
\begin{aligned}
\mu_{a \mathrm{~T}_{30}} & =\frac{A_{\mathrm{T}_{30}}}{h_{\mathrm{T}_{30}}} \\
& =\varepsilon_{\mathrm{T}_{30}} c_{\mathrm{T}_{30}}
\end{aligned}
$$


However, the absorption coefficient at $30^{\circ} \mathrm{C}$ is now being expressed at the concentration at $30^{\circ} \mathrm{C}$ and as a result cannot be directly compared to $\mu_{a} \mathrm{~T}_{40}$ since this was expressed at the concentration at $40^{\circ} \mathrm{C}$. Since concentration and height are inversely proportional to one another then it follows that $c_{\mathrm{T}_{30}} h_{1 \mathrm{~T}_{30}}=c_{\mathrm{T}_{40}} h_{1 \mathrm{~T}_{40}}$, thus attenuation at $30^{\circ} \mathrm{C}$ can also be given by

$$
A_{\mathrm{T}_{30}}=\varepsilon_{\mathrm{T}_{30}} c_{\mathrm{T}_{40}} h_{1 \mathrm{~T}_{40}}
$$

To express the absorption coefficient measured at $30^{\circ} \mathrm{C}$ at the water concentration existing at $40^{\circ} \mathrm{C}$, therefore, the following calculation is made

$$
\begin{aligned}
\mu_{a \mathrm{~T}_{30}} & =\frac{A_{\mathrm{T}_{30}}}{h_{1 \mathrm{~T}_{40}}} \\
& =\varepsilon_{\mathrm{T}_{30}} c_{\mathrm{T}_{40}}
\end{aligned}
$$

This example demonstrates that no correction to the original height is required to calculate $\mu_{a}$ at subsequent temperatures. The absorption coefficient calculated at any temperature is standardised to the water concentration at the temperature at which the initial volume was added, $40^{\circ} \mathrm{C}$ in the above example. Often, though, it may be useful to standardise $\mu_{a}$ to a concentration other than that at the temperature at which the initial volume was added, e.g. $37^{\circ} \mathrm{C}$. In the general case then, it follows from Equation (5.7) that the attenuation at any temperature $\mathrm{T}$ can be given by

$$
A_{\mathrm{T}}=\varepsilon_{\mathrm{T}} c_{\mathrm{T}_{S}} h_{1 \mathrm{~T}_{S}}
$$

where $c_{\mathrm{T}_{S}}$ and $h_{1 \mathrm{~T}_{S}}$ are the concentration and height of water at the 'standardising' temperature $T_{S}$ respectively. Therefore, the absorption coefficient at any temperature $\mathrm{T}$, expressed at the concentration at the standardising temperature, is given by

$$
\begin{aligned}
\mu_{a \mathrm{~T}} & =\frac{A_{\mathrm{T}}}{h_{1 \mathrm{~T}_{S}}} \\
& =\varepsilon_{\mathrm{T}} c_{\mathrm{T}_{S}}
\end{aligned}
$$

where $h_{1} \mathrm{~T}_{S}$ is determined as follows

$$
h_{1 \mathrm{~T}_{S}}=\frac{h_{1 \mathrm{~T}_{1}} d_{\mathrm{T}_{1}}}{d_{\mathrm{T}_{S}}}
$$

and where $h_{1 \mathrm{~T}_{1}}$ and $d_{\mathrm{T}_{1}}$ are the height and density of the water at a temperature $\mathrm{T}_{1}$, the temperature of the water when the initial volume $V_{1}$ was added, and $d_{\mathrm{T}_{S}}$ is the density 
of water at the standardising temperature. As long as the temperature at which the water was added is known, therefore, one can calculate the absorption coefficient at any temperature, standardised to the concentration at any fixed temperature using this vertical alignment set-up.

As described in Section 5.1.2.1, Method I involved making an initial reference intensity measurement in order to reduce refractive index mismatches. This measurement also accounts for the photometric response of the system (see Section 3.1), which should effectively be cancelled out in the calculation of attenuation. For the reference measurement an initial volume of water $V_{1}$ was added to give a height $h_{1}(1 \mathrm{~cm}$ at the temperature at which the water was added, $\mathrm{T}_{1}$ ) and a reference intensity $I_{r}$ was recorded at a temperature $\mathrm{T}_{r}$, approximately $44^{\circ} \mathrm{C}$. An additional volume $V_{2}$ was then added, the additional height $h_{2}$ varying according to wavelength range measured (see Table 5.1 in Section 5.1.2.1), and 'absolute' measurements were subsequently made. At the temperature at which the reference measurement was made, $\mathrm{T}_{r}$, the attenuation through the additional volume of water is calculated as

$$
A_{\mathrm{T}_{r}}=\log _{10} \frac{I_{r \mathrm{~T}_{r}}}{I_{f} \mathrm{~T}_{r}}
$$

where $I_{f} \mathrm{~T}_{r}$ is the intensity measured through the full height of water, from which it follows that the absorption coefficient at temperature $\mathrm{T}_{r}$ (standardised to the concentration at temperature $\mathrm{T}_{S}$ ) would be given by

$$
\mu_{a \mathrm{~T}_{r}}=\frac{1}{h_{2 \mathrm{~T}_{S}}} \log _{10} \frac{I_{r} \mathrm{~T}_{r}}{I_{f} \mathrm{~T}_{r}}
$$

where $h_{2} \mathrm{~T}_{S}$ can be calculated using Equation (5.11). However, if the water was cooled to any other temperature $\mathrm{T}$, the calculation of $\mu_{a}$ in Equation (5.13) would no longer be valid since the calculation uses the reference intensity measured at temperature $\mathrm{T}_{r}$, but the temperature of the reference volume would also have changed. In other words, due to the effect of temperature on the water absorption coefficient, part of the measured system response will change with temperature. The following steps have therefore been developed to correct for this effect and calculate the absorption coefficient at any temperature.

The attenuation through the glass tube containing the full water height $h_{1}+h_{2}$ at any temperature $\mathrm{T}$ can be defined as

$$
A_{f \mathrm{~T}}=\log _{10} \frac{I_{0}}{I_{f} \mathrm{~T}}
$$




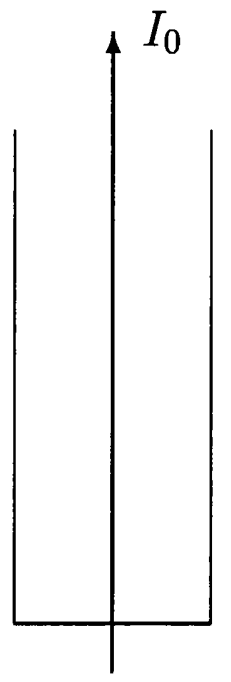

A

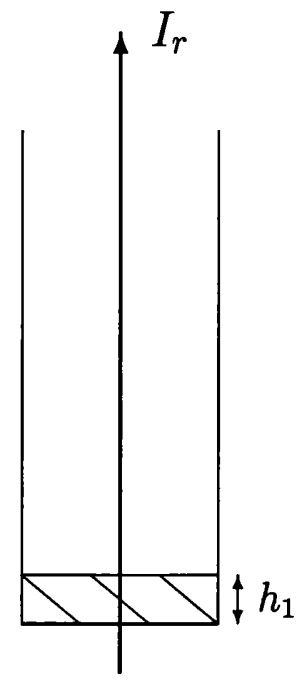

B

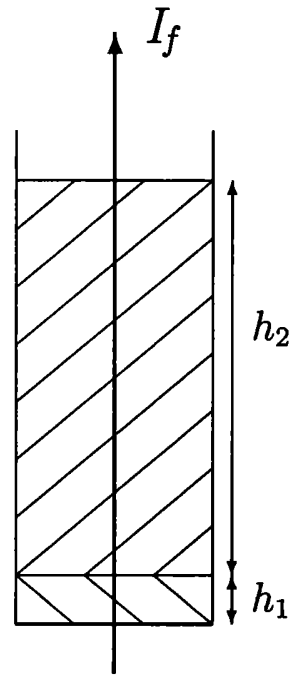

C

Figure 5.2: Definition of intensity measurements in determination of water absorption spectrum using Method I. A: Intensity $I_{0}$ measured through empty glass tube. B: Reference intensity $I_{r}$ measured through water height $h_{1}$. C: Intensity $I_{f}$ measured through full height $h_{1}+h_{2}$.

where $I_{0}$ is the intensity emerging from the empty tube and $I_{f} \mathrm{~T}$ is the intensity measured through the full height of water at temperature $\mathrm{T}$ (see Figure 5.2, parts $\mathbf{A}$ and $\mathbf{C}$ ). The absorption coefficient at temperature $\mathrm{T}$ (standardised to the water concentration at temperature $\mathrm{T}_{S}$ ) would then be

$$
\begin{aligned}
\mu_{a \mathrm{~T}} & =\frac{1}{h_{1 \mathrm{~T}_{S}}+h_{2 \mathrm{~T}_{S}}}\left(A_{f \mathrm{~T}}+\delta\right) \\
& =\frac{1}{h_{1 \mathrm{~T}_{S}}+h_{2 \mathrm{~T}_{S}}}\left(\log _{10} \frac{I_{0}}{I_{f} \mathrm{~T}}+\delta\right)
\end{aligned}
$$

where $\delta$ is an error due to the differences in reflectance at the glass-air interface in the measurement of $I_{0}$ and the glass-water and water-air interfaces in the measurement of $I_{f}$. (The concept of a standardising concentration is particularly useful when more than one volume of water is added, possibly at different temperatures, as is the case here.) Similarly, the attenuation through the initial reference height $h_{1}$ (see part $\mathbf{B}$ of Figure 5.2), measured at the reference temperature $\mathrm{T}_{r}$, can be defined as

$$
A_{r \mathrm{~T}_{r}}=\log _{10} \frac{I_{0}}{I_{r \mathrm{~T}_{r}}}
$$


with the absorption coefficient at $\mathrm{T}_{r}$ given by

$$
\begin{aligned}
\mu_{a \mathrm{~T}_{r}} & =\frac{1}{h_{1 \mathrm{~T}_{S}}}\left(A_{r \mathrm{~T}_{r}}+\delta\right) \\
& =\frac{1}{h_{1 \mathrm{~T}_{S}}}\left(\log _{10} \frac{I_{0}}{I_{r} \mathrm{~T}_{r}}+\delta\right)
\end{aligned}
$$

It should be noted that the $\delta$ here will be slightly different to the one expressed in Equation (5.15) due to the change in the refractive index of water with temperature. Reflectance at normal incidence is given by

$$
r_{\perp}=\left(\frac{n_{2}-n_{1}}{n_{2}+n_{1}}\right)^{2}
$$

where $n_{1}$ and $n_{2}$ are the refractive indices of the first and second media respectively. As an approximate guide, the difference in reflectance at $589 \mathrm{~nm}$ for normal incidence on a glass-to-water boundary at $44^{\circ} \mathrm{C}$ and at $30^{\circ} \mathrm{C}$ was calculated to be less than $0.01 \%$. Hence, the errors $\delta$ in Equations (5.15) and (5.17) can be assumed to be the same. Another definition of $\mu_{a}$ at temperature $\mathrm{T}_{r}$ was given in Equation (5.13), in which the measurement $I_{r}$ through an initial height $h_{1}$ of water was used as a reference and hence no correction for refractive index mismatches was required. This relationship can therefore be used to eliminate the unknowns, $I_{0}$ and $\delta$, in Equation (5.15). Rearranging Equation (5.17) to give the unknowns we have

$$
\log _{10} I_{0}+\delta=h_{1 \mathrm{~T}_{S}} \mu_{a \mathrm{~T}_{r}}+\log _{10} I_{r} \mathrm{~T}_{r}
$$

Substituting $\mu_{a} \mathrm{~T}_{r}$ from Equation (5.13) into Equation (5.19) gives

$$
\log _{10} I_{0}+\delta=\frac{h_{1 \mathrm{~T}_{S}}}{h_{2} \mathrm{~T}_{S}} \log _{10} \frac{I_{r \mathrm{~T}_{r}}}{I_{f} \mathrm{~T}_{r}}+\log _{10} I_{r} \mathrm{~T}_{r}
$$

Substituting the above expression for the unknowns into Equation (5.15) gives the absorption coefficient at any temperature $\mathrm{T}$ (standardised to the concentration at temperature $\left.\mathrm{T}_{S}\right)$

$$
\begin{aligned}
\mu_{a \mathrm{~T}} & =\frac{1}{h_{1 \mathrm{~T}_{S}}+h_{2 \mathrm{~T}_{S}}}\left(\log _{10} I_{0}-\log _{10} I_{f \mathrm{~T}}+\delta\right) \\
& =\frac{1}{h_{1 \mathrm{~T}_{S}}+h_{2 \mathrm{~T}_{S}}}\left(\frac{h_{1 \mathrm{~T}_{S}}}{h_{2 \mathrm{~T}_{S}}} \log _{10} \frac{I_{r \mathrm{~T}_{r}}}{I_{f} \mathrm{~T}_{r}}+\log _{10} \frac{I_{r} \mathrm{~T}_{r}}{I_{f \mathrm{~T}}}\right)
\end{aligned}
$$

Thus, the water absorption coefficient as a function of temperature can be calculated 
from these experimental measurements of intensity, using a reference at a single temperature, without knowledge of the input light intensity $I_{0}$ or errors due to differences in reflectances $\delta$. (In the previously mentioned publication (Hollis et al, 2001) the absorption coefficient was corrected to the concentration at temperature $\mathrm{T}$ instead of to a fixed, standardising temperature $\mathrm{T}_{S}$. The calculated absorption coefficient was thus in error by a factor of $d_{\mathrm{T}} / d_{\mathrm{T}_{S}}$, approximately $0.2 \%$ at $30^{\circ} \mathrm{C}$ with a standardising temperature of $37^{\circ} \mathrm{C}$.)

As described in Section 5.1.2.1, the acquisition software averages together contiguous spectra in order to reduce the data storage to a reasonable rate and at the same time improve the SNR. The averaged data were exported from the acquisition software by a custom-written program which further averaged the data such that the maximum temperature change between consecutive spectra was $0.05^{\circ} \mathrm{C}$, as estimated from the slope of the original temperature data with respect to time. Again, this reduced the size of the data set and the noise on each spectrum. The data were imported into MATLAB ${ }^{\circledR}$ and the absorption spectra were calculated using Equation (5.21).

The absorption spectra for the three NIR water bands measured as a function of temperature are displayed in Figures 5.3-5.5. The temperature at which the displayed spectra were measured is indicated by the adjacent colour bar, and the arrow shows the direction of decreasing temperature. The measured ranges have been cropped slightly to best dis-

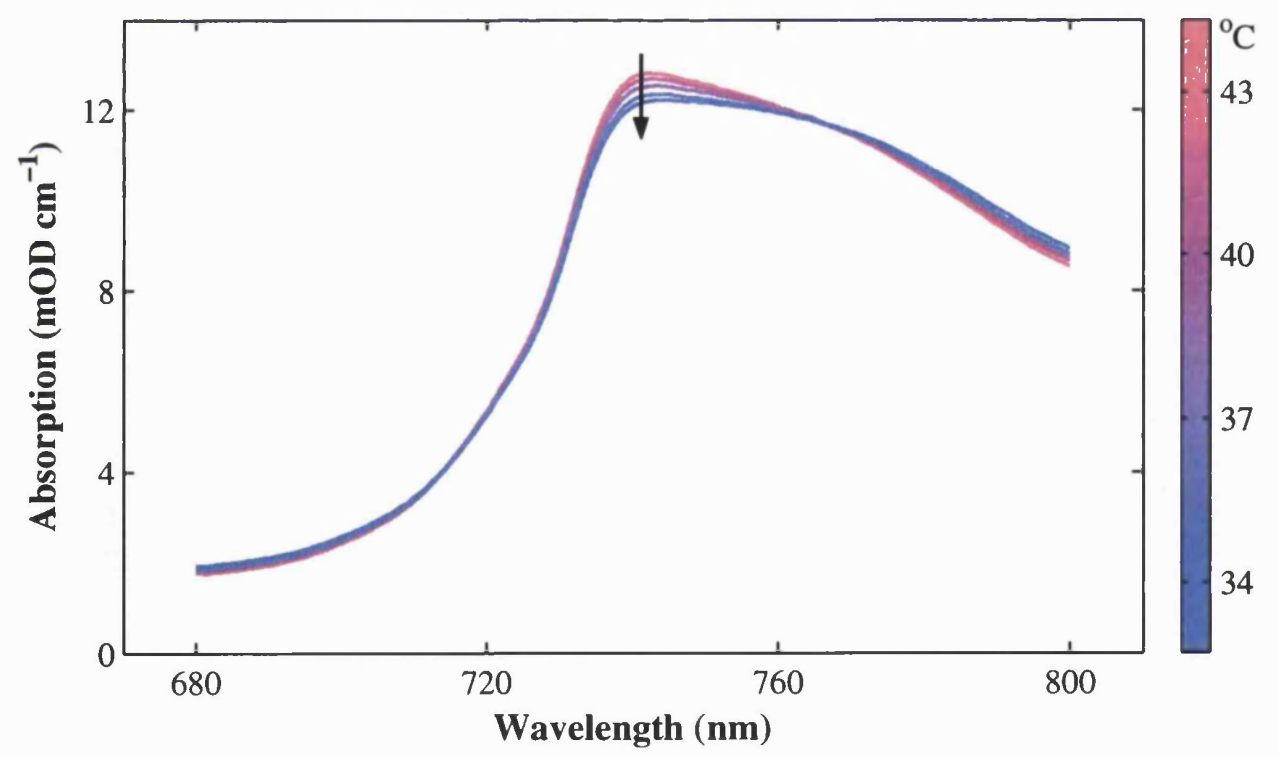

Figure 5.3: NIR absorption coefficient of the $740 \mathrm{~nm}$ water absorption band measured using Method I as a function of temperature between 44 and $30^{\circ} \mathrm{C}$ - arrow indicates direction of decreasing temperature. For clearer display the measured range has been cropped and the spectra have been smoothed with a filter (see text for details). 


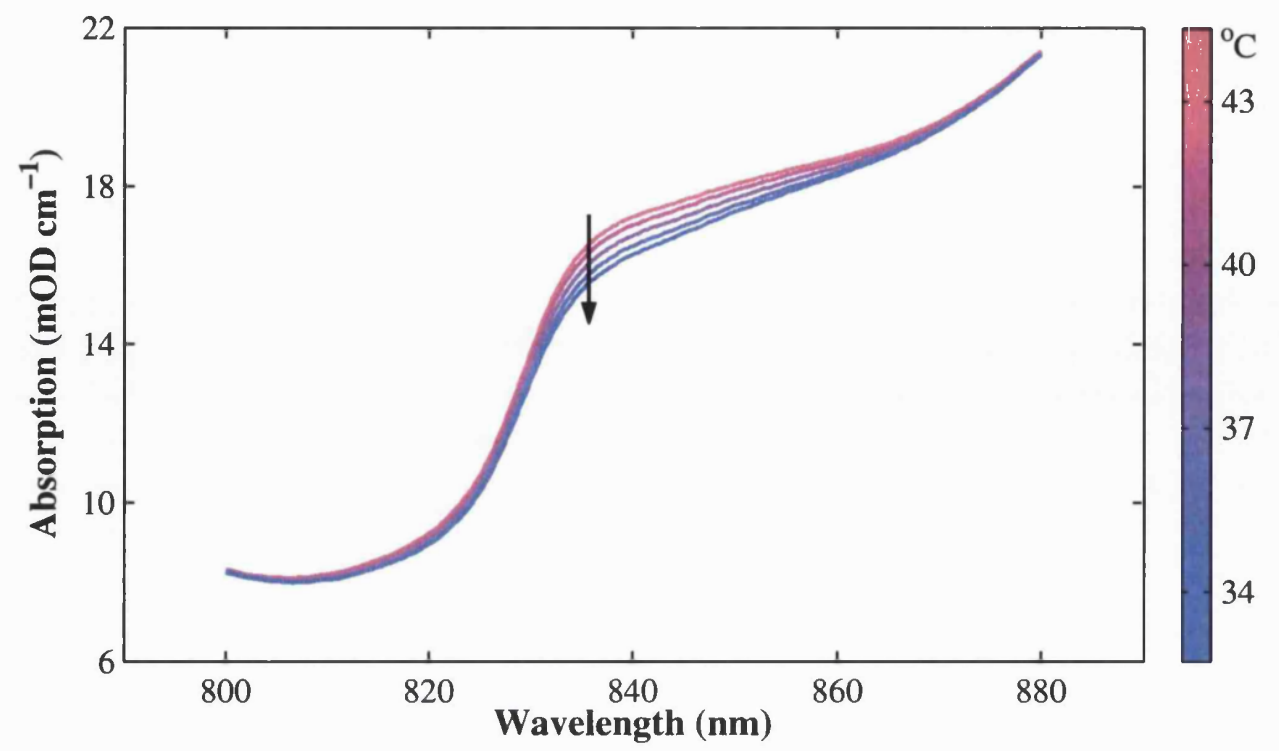

Figure 5.4: NIR absorption coefficient in the region of the $840 \mathrm{~nm}$ water absorption band measured using Method I as a function of temperature between 44 and $30^{\circ} \mathrm{C}$ - arrow indicates direction of decreasing temperature. For clearer display the measured range has been cropped and the spectra have been smoothed with a filter (see text for details).

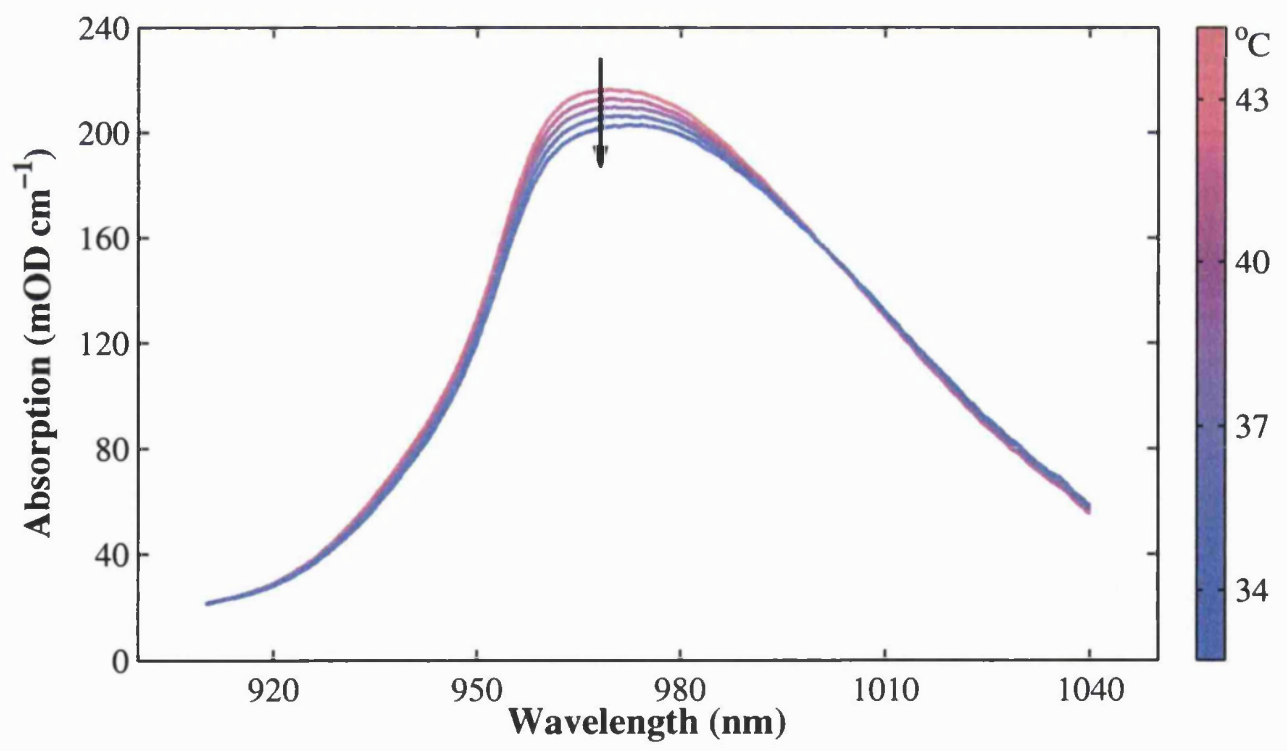

Figure 5.5: NIR absorption coefficient in the region of the $970 \mathrm{~nm}$ water absorption band measured using Method I as a function of temperature between 44 and $30^{\circ} \mathrm{C}$ - arrow indicates direction of decreasing temperature. For clearer display the measured range has been cropped and the spectra have been smoothed with a filter (see text for details). 
play the temperature response of the water bands. For clearer display the spectra were also smoothed by a Savitsky-Golay filter, the details of which will be described in Section 7.1.2. Due to the wavelength-dependence of dispersion, the wavelength separation between spectral points was not constant over the whole range. In order that the filter could be applied, therefore, the spectra were interpolated such that the wavelength separation between consecutive points was a constant $0.15 \mathrm{~nm}$ for all ranges. Each of the NIR water bands decreases in amplitude and their peak positions appear to shift towards longer wavelengths with decreasing temperature. The decreases in amplitude are approximately $0.5-0.8 \%{ }^{\circ} \mathrm{C}^{-1}$ (see also Section 5.1.3) and the shifts in position (easiest to see in the 970 $\mathrm{nm}$ band) are approximately $5-10 \mathrm{~nm}$ over the whole temperature range.

One particular problem associated with Method I is the necessity that the measurements $I_{r}$ and $I_{f}$ are made at the exact same temperature (within $\pm 0.2^{\circ} \mathrm{C}$ ), in order to determine absolute $\mu_{a}$ as a function of temperature, as given in Equation (5.21). As mentioned in Section 5.1.2.1, to avoid disturbing the temperature inside the incubator and hence in the water column, the additional volume of water was delivered to the glass tube via a catheter fed through a small hole in the incubator wall. Moreover, the water added was allowed to warm up inside the incubator during heating of the reference volume. Even though these precautions were taken, the temperature of water inside the tube took a considerable amount of time to return to the temperature at which the reference measurement was made. The concern is that during this time the output intensity of the light source may drift (also a potential problem during the experiment itself), which will not be accounted for since only one reference measurement is made. Furthermore, the longer the water is maintained at high temperatures the greater the chance of water evaporation occurring. To avoid these effects the measurements should be made in as short a time as possible, without compromising the temperature resolution or SNR.

Another problem associated with Method I was discovered later on close inspection of the absorption spectra. Figure 5.6 shows the measurements of the $840 \mathrm{~nm}$ and $970 \mathrm{~nm}$ absorption bands in the range $895-920 \mathrm{~nm}$, at a temperature of $40^{\circ} \mathrm{C}$. An absorption feature can be seen at around $910 \mathrm{~nm}$ that appears more significant in the measurement of the $970 \mathrm{~nm}$ band. Because the feature is 'negative', this indicates that the absorption in this wavelength region $(\sim 905-915)$ has decreased between the reference and absolute measurements. After extensive checking of the experimental set-up and methodology had eliminated potential candidates, it became apparent that the thin layer of silicon oil added to reduce water evaporation was responsible for this phenomenon. The additional volume 


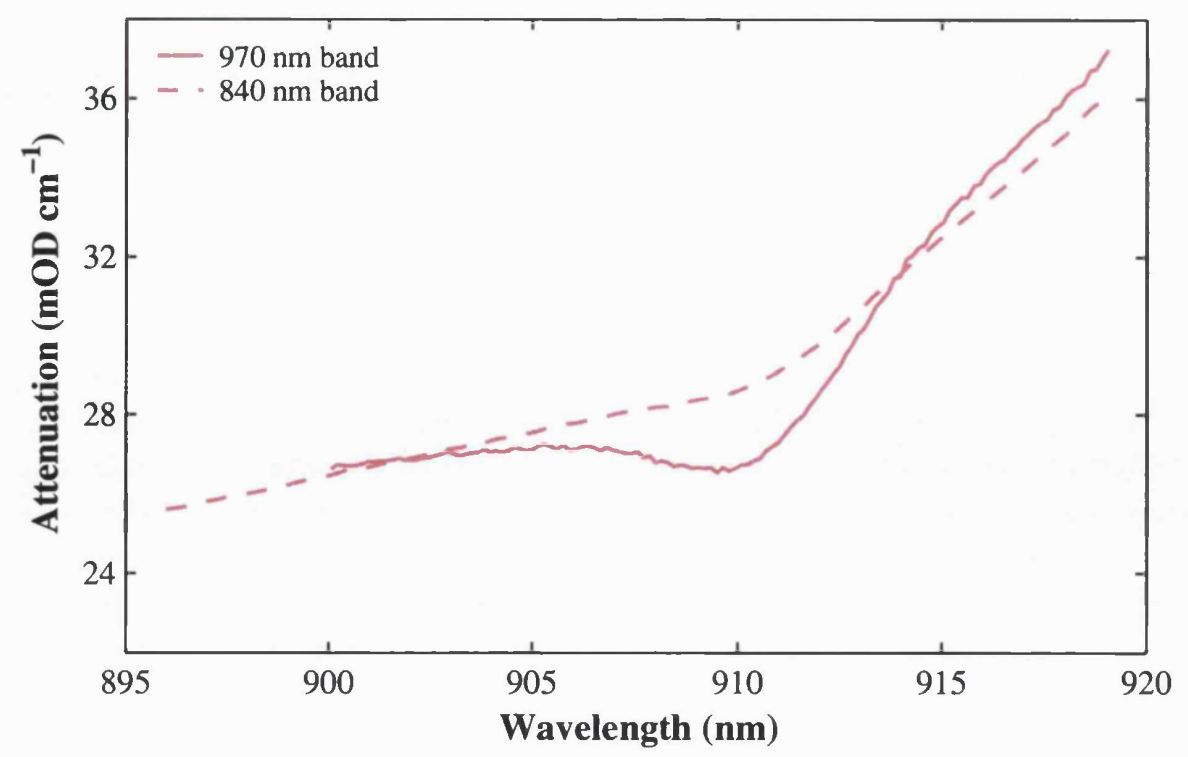

Figure 5.6: Close-up display of the $840 \mathrm{~nm}$ and $970 \mathrm{~nm}$ water absorption bands in the range $895-920 \mathrm{~nm}$ measured at $40^{\circ} \mathrm{C}$ using Method I. Note the absorption feature positioned around $910 \mathrm{~nm}$, which is more significant in the measurement of the $970 \mathrm{~nm}$ band (see text for details).

of water for the absolute measurements is added underneath the oil layer, by careful insertion of the catheter to the bottom of the glass tube. Although difficult to observe by eye, it seems probable that as the water is delivered and the level in the tube rises, a small amount of the oil adheres to the sides of the glass tube, thus depleting the layer floating on top of the water. Hence the oil layer in the absolute measurement is thinner than during the reference measurement. This means its contribution to the total absorbance does not cancel out in the calculation of the water absorption coefficient, and its (negative) presence can therefore be seen in the water spectrum, as shown in Figure 5.6.

This suggestion was confirmed by measuring the absorption spectrum of the oil itself, shown in Figure 5.7. (The unit of the $y$-axis in Figure 5.7 is attenuation as opposed to absorption because the reference measurement was made through the empty tube and the spectrum therefore has an unknown offset due to refractive index mismatches. The aim, however, was to determine the shape of the absorption spectrum, not the absolute absorption coefficient.) The oil spectrum reveals a large absorption peak positioned at exactly $910 \mathrm{~nm}$, and a smaller feature at about $745 \mathrm{~nm}$. To further confirm that the absorption feature seen in Figure 5.6 was due to the decrease in the amount of silicone oil, the water absorption spectrum was also inspected in the region around $745 \mathrm{~nm}$. Figure 5.8 shows the second derivative of the measured water spectrum and an additional water spectrum measured in the absence of the silicone oil layer, in the range 700-915 $\mathrm{nm}$. The 


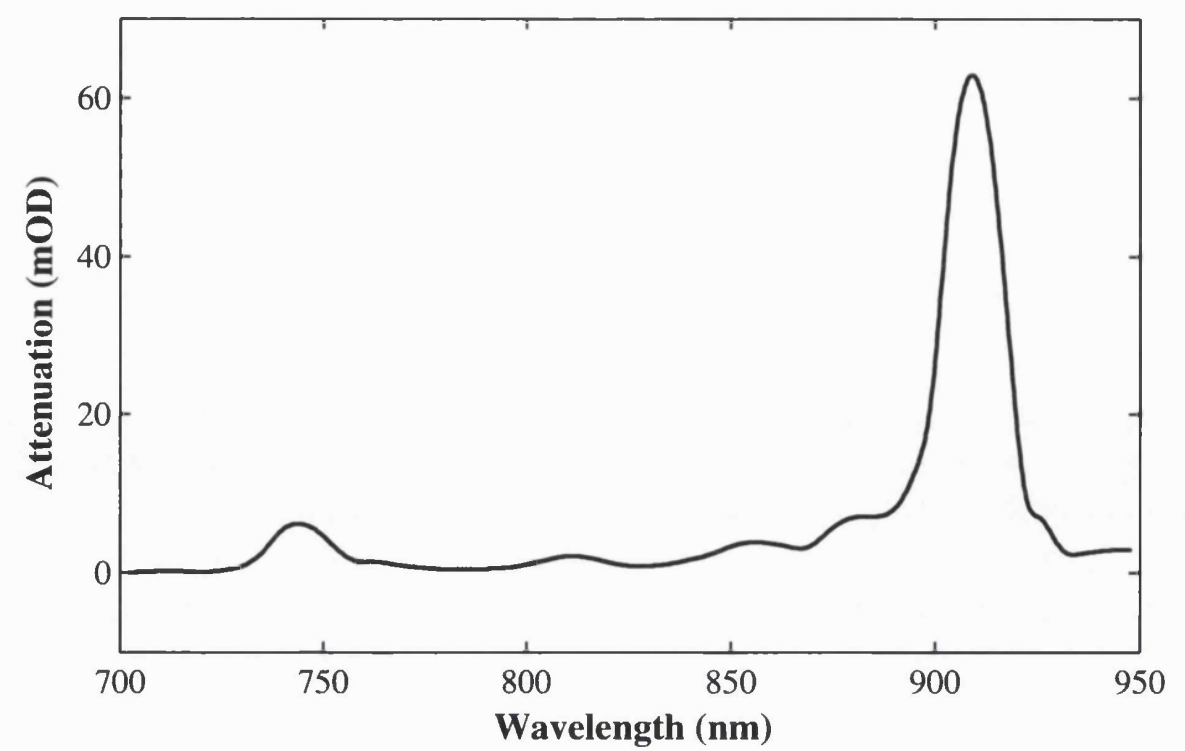

Figure 5.7: Attenuation measured through $1 \mathrm{~cm}$ of silicone oil in the range 700-950 nm (arbitrarily zeroed at $700 \mathrm{~nm}$ ). Note the large absorption feature centred at around 910 $\mathrm{nm}$ and the smaller feature at approximately $745 \mathrm{~nm}$.

second derivative was used to compare the spectra as it more clearly shows the underlying absorption bands that may overlap one another, e.g. the $740 \mathrm{~nm}$ water band and the $745 \mathrm{~nm}$ oil band (see Section 7.1). Both the 745-750 nm and 905-910 nm features of the oil spectrum can be seen in the second derivative spectrum of the measurement with the oil layer, whereas they do not appear in the measurement without the oil layer.

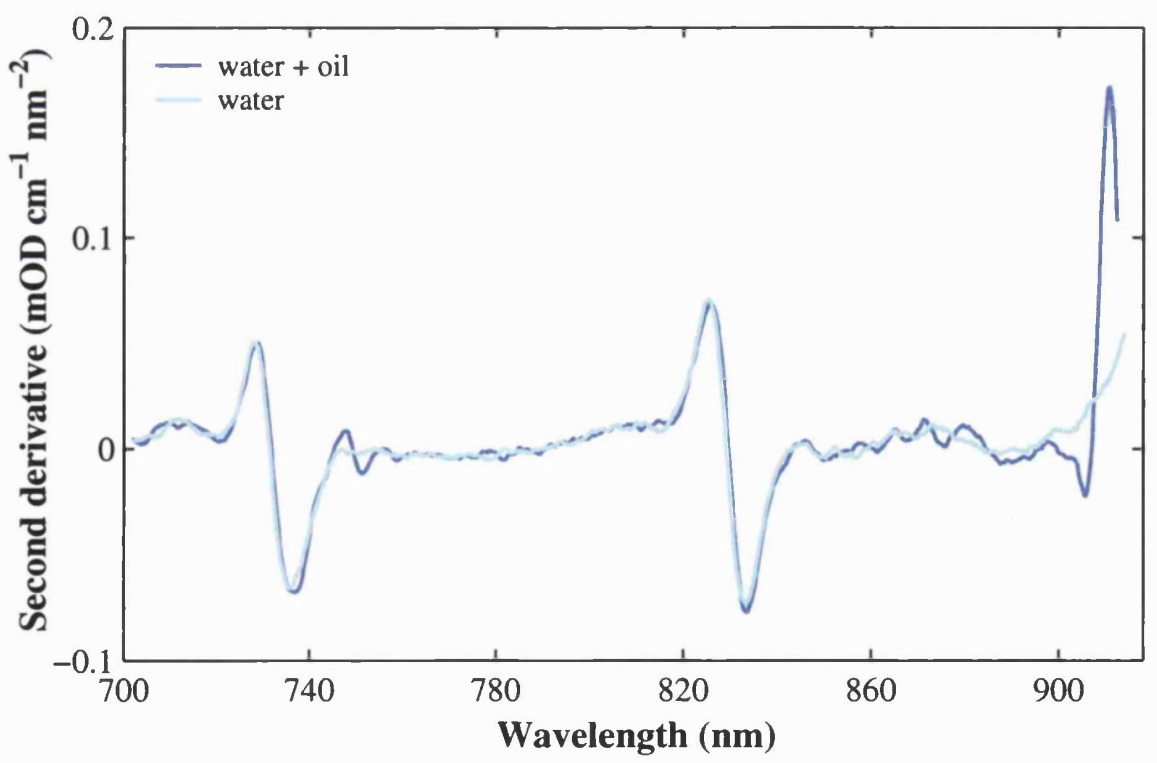

Figure 5.8: Second derivative comparison of measurements of water absorption with and without oil layer in the range $700-915 \mathrm{~nm}$. Note the features at approximately 750 and $905 \mathrm{~nm}$ in the measurement of the water spectrum with the oil layer and the absence of these features in the measurement without the oil layer. 
Method I thus has two major disadvantages:

i) in order to obtain absolute measurements of water absorption as a function of temperature the water temperature must return to that of the reference measurement after addition of the second volume of water, which extends the time taken for the experiment

ii) the oil added to reduce the amount of water evaporation interferes with the measurement of water absorption by contributing less to the total absorption in the absolute measurement, due to oil adhering to the walls of the glass tube.

The second point listed above was not discovered until after the temperature prediction algorithms (Sections 7.1 and 7.2) which use the temperature calibration of this data (Section 5.3.5) were developed. However, as will be discussed in Sections 7.1 and 7.2 the wavelength range used did not extend beyond $880 \mathrm{~nm}$, hence the oil feature at $745 \mathrm{~nm}$ (barely visible in Figure 5.3) is the only interfering factor, and due to its relatively small size (compared to the water absorption) is likely to have a minimal effect on the analysis.

As a result of the problems associated with Method I a second method was developed, which shall be used in the later temperature calibration and prediction algorithms, discussed in Sections 5.3.6 and 7.3. The following sub-sections will discuss the methodology and results of Method II.

\subsubsection{Method II}

The new methodology essentially followed the same experimental design as Method I, described in Section 5.1.1, and the methodological elements common to both methods are listed in Section 5.1.2. Method II is therefore based on the following improvements to Method I.

Firstly, the water absorption bands positioned at 740 and $840 \mathrm{~nm}$ were measured simultaneously in an extended range from 600 to $925 \mathrm{~nm}$. As will be explained in later sections, in order to improve the temperature prediction, much of the further analysis involves the use of both the 740 and the $840 \mathrm{~nm}$ water absorption bands. For the analysis employing the results of Method I, this required that the two ranges covering the 740 and $840 \mathrm{~nm}$ bands were matched together in the overlapping region of 765-805 $\mathrm{nm}$. Matching the ranges together was a somewhat arbitrary process, since the spectra in the overlap region were offset from one another by $\sim 4-7 \%$ with a slight wavelength dependence to 
the offset. For the further analysis using this data the spectra of the first measured range (650-805 $\mathrm{nm}$ ) were offset (and slightly scaled) to match those in the second range (765-920 $\mathrm{nm}$ ) in the overlap region. The reason for this choice was the fact that the time taken for the measurements of the second range was reduced due to a more efficient practice, thus reducing the potential for instrumental drift. Moreover, the second range matched well to the third range $(900-1050 \mathrm{~nm})$, as can be seen in Figure 5.6. It is far more practical, however, to measure the two bands together in one range. This was done in Method II using the 300 grooves $\mathrm{mm}^{-1}$ dispersion grating, which, with the spectrograph slit width maintained at $100 \mu \mathrm{m}$, halved the spectral resolution compared to that for the single band measurement (see Section 5.1.2.1). The advantage in measuring the 740 and $840 \mathrm{~nm}$ bands simultaneously far outweighs the small loss in resolution, which is still extremely high $(1.24 \mathrm{~nm})$ compared to the width of the water absorption bands. Hence the measured ranges were $600-925 \mathrm{~nm}$ and $900-1050 \mathrm{~nm}$. For the 900-1050 nm range, measured using the 600 grooves $\mathrm{mm}^{-1}$ grating as before, the slit width was increased to $200 \mu \mathrm{m}$ in order to obtain the same resolution as the $600-925 \mathrm{~nm}$ range.

As with Method I the spectral measurements were made during cooling, in the temperature range $41-29^{\circ} \mathrm{C}$. In Method II, however, the reference measurement was performed through the smallest possible volume of water that would cover the bottom of the tube, approximately $0.4 \mathrm{ml}$ corresponding to a height of $1.2 \mathrm{~mm}$. The aim of this step was to prevent refractive index mismatches as before, but ensure that the reference volume was small enough such that the correction for the change in its absorption with temperature (Equations (5.12)-(5.21)) could be ignored. The error in the calculation of $\mu_{a}$ incurred by this step will be discussed in Section 5.1.2.4. The incubator was set to $40^{\circ} \mathrm{C}$ and when the temperature of an equivalent volume of water (heated inside the incubator) to that in the delivery syringe reached a stable value at around $40^{\circ} \mathrm{C}$, as measured by a mercury thermometer, the reference volume of water was added from the syringe (also heating inside the incubator). The point of heating the water prior to adding the reference volume was not so much for the purpose of performing the reference measurement at exactly $40^{\circ} \mathrm{C}$, since no temperature correction will be made for this measurement, but more to reduce the time between adding the reference volume and the additional volume. In other words, the reference volume could be added at any temperature, but if added at room temperature the incubator would then be required to begin heating the additional volume of water in the syringe to around $40^{\circ} \mathrm{C}$, during which time the small reference volume inside the tube could have partially evaporated and perturbed the absorption of the light. 


\begin{tabular}{llll}
\hline $\begin{array}{l}\text { Measured } \\
\text { band }(\mathrm{nm})\end{array}$ & $\begin{array}{l}\text { Absolute } \\
\text { height }(\mathrm{cm})\end{array}$ & $\begin{array}{l}\text { Acquisition } \\
\text { time (ms) }\end{array}$ & $\begin{array}{l}\text { Averaging } \\
\text { factor }\end{array}$ \\
\hline 740 \& 840 & 14 & 500 & 4 \\
970 & 4 & 700 & 3 \\
\hline
\end{tabular}

Table 5.2: Experimental and acquisition parameters used during the measurement of water absorption by Method II

In Method II no silicone oil layer was added to the reference volume, because of the problems associated with this discussed in Section 5.1.2.2. The absorption spectra of several other oils (coconut, linseed, sunflower and paraffin) were also measured but all contained significant absorption bands at around 740-760 and 910-930 nm. Since nothing was to prevent the water from evaporating during the experiment, it was necessary to minimise the time taken for the whole experiment. To obtain an estimate of the extent of the evaporation during the experiment, a measuring cylinder with a diameter similar to that of the glass tube was filled with the same volume of water, the mass of which was measured when adding the additional volume to the glass tube and again at the end of the experiment. The rate of water evaporation from a vessel (for a given temperature, humidity, salinity and so on) depends on the surface area-to-volume ratio. For a cylinder of slightly larger diameter than the glass tube (22.5 mm as opposed to $20.6 \mathrm{~mm})$ the evaporation rate will be greater for the same volume of water, and the smaller the volume, the greater the rate of evaporation. Of the two measured wavelength ranges, the volume of water was smallest for the measurement of the 900-1050 nm range. During this experiment water evaporation from the measuring cylinder equated to a total volume (and therefore height) loss of $0.4 \%$, which is likely to be an overestimation of the water loss in the glass tube. Moreover, the water evaporation in the measurement of the $600-925 \mathrm{~nm}$ range will be smaller again, because of the greater volume added. Since the percentage volume loss due to water evaporation during these experiments is smaller than the reported accuracy of the delivery pump, its effect on the calculation of the absorption coefficient will be disregarded.

After adding the reference volume the system was optically aligned as described in Section 5.1.2.1. The incubator was made light-tight and the reference measurement was performed. The additional volume was added immediately after the reference measurement, recording the exact temperature at the time, as before. For the measurement of the extended range $(600-925 \mathrm{~nm})$ the additional volume corresponded to a height of 14 $\mathrm{cm}$ and for the 900-1050 $\mathrm{nm}$ range the extra height was $4 \mathrm{~cm}$, as opposed to $12 \mathrm{~cm}$ and 1 
$\mathrm{cm}$ for these ranges respectively in Method I (see Table 5.1). The additional heights were increased in order to maximise the measured change in absorbance and thereby reduce the errors in the calculation of $\mu_{a}$ (see Section 5.1.3). After allowing the water temperature inside the tube to stabilise for approximately 10-15 minutes, spectral measurements began. Table 5.2 shows the spectral acquisition parameters for measurements obtained using Method II. The incubator temperature was set to $20^{\circ} \mathrm{C}$ after approximately 2 minutes of acquisition at $40^{\circ} \mathrm{C}$. Setting the incubator temperature to $20^{\circ} \mathrm{C}$, as opposed to $30^{\circ} \mathrm{C}$ with Method I, reduced the time taken for the whole experiment ( $\sim 3.5$ hours) but at the expense of temperature resolution, as will be discussed in Section 5.1.3.

\subsubsection{Analysis and Results II}

The measurements of the water spectrum performed using Method II were exported from the acquisition software and imported into MATLAB ${ }^{\circledR}$, again averaged such that the maximum temperature difference between spectra was $0.05^{\circ} \mathrm{C}$. Because the reference measurement was small enough to ignore, the absorption coefficient as a function of temperature was calculated as follows using the formulation described by Equations (5.3)-(5.11)

$$
\mu_{a \mathrm{~T}}=\frac{1}{h_{2 \mathrm{~T}_{S}}} \log _{10} \frac{I_{r}}{I_{f} \mathrm{~T}}
$$

where $I_{r}$ is the temperature-independent reference measurement and $\mathrm{T}_{S}$ is as before the standardising temperature $\left(37^{\circ} \mathrm{C}\right)$.

The difference between the absorption coefficient calculated by Equation (5.22) for Method II and Equation (5.21) for Method I is given by

$$
\mu_{a \mathrm{~T}}^{\mathrm{II}}-\mu_{a \mathrm{~T}}^{\mathrm{I}}=\frac{h_{1 \mathrm{~T}_{S}}}{h_{2 \mathrm{~T}_{S}}\left(h_{1 \mathrm{~T}_{S}}+h_{2 \mathrm{~T}_{S}}\right)} \log _{10} \frac{I_{f} \mathrm{~T}_{r}}{I_{f \mathrm{~T}}}
$$

At $30^{\circ} \mathrm{C}$ this difference amounts to a percentage error in $\mu_{a \mathrm{~T}}^{\mathrm{II}}$ of $< \pm 0.01 \%$, i.e. this is the error produced by ignoring the reference volume, which is negligible compared to other sources of error in these measurements (see Section 5.1.3).

The absorption spectra measured using Method II in the temperature range $41-29^{\circ} \mathrm{C}$ and calculated using Equation (5.22) are displayed in Figures 5.9 and 5.10. As before the spectra were smoothed with a filter for clearer display and the ranges cropped slightly. In further analysis of the $600-925 \mathrm{~nm}$ range the data below $650 \mathrm{~nm}$ was excluded due to the effects of the $610 \mathrm{~nm}$ long-pass filter. 


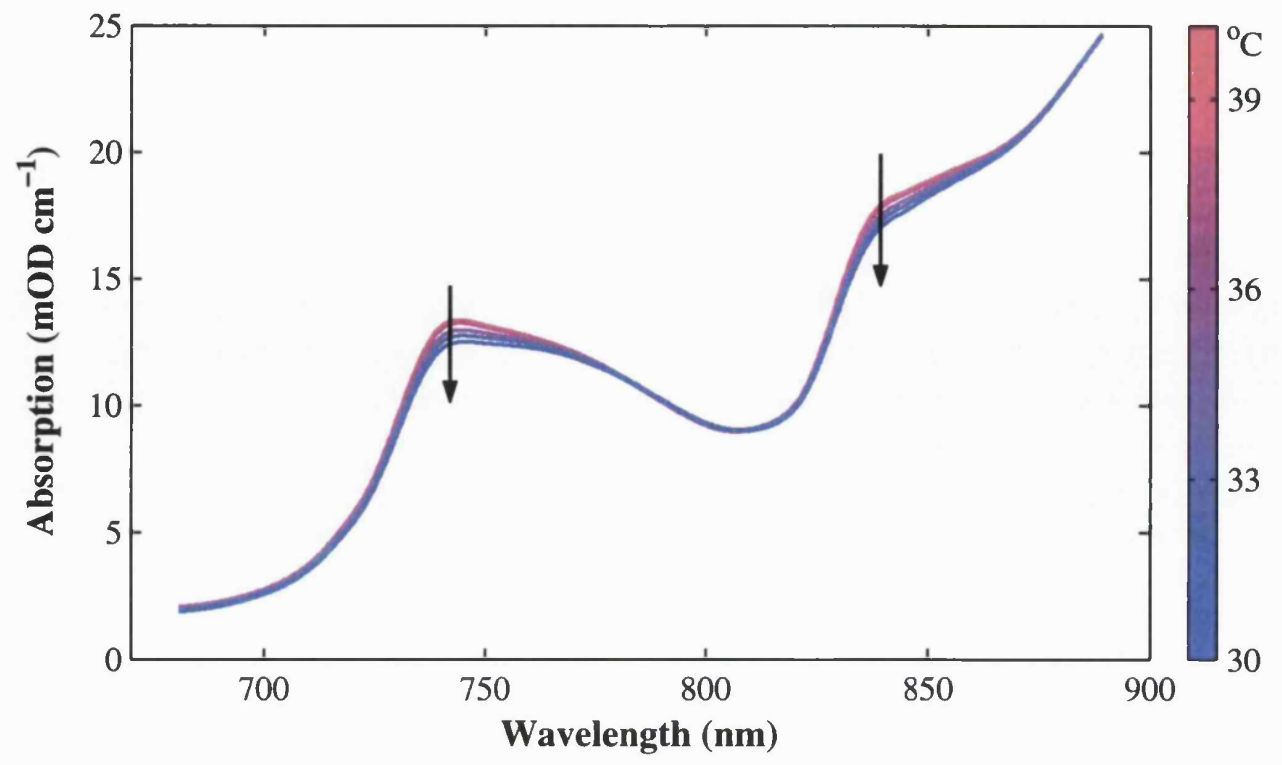

Figure 5.9: NIR absorption coefficient of the 740 and $840 \mathrm{~nm}$ water absorption bands measured using Method II as a function of temperature between 40 and $30^{\circ} \mathrm{C}$ - arrows indicate direction of decreasing temperature. For clearer display the measured range has been cropped and the spectra have been smoothed with a filter (see text for details).

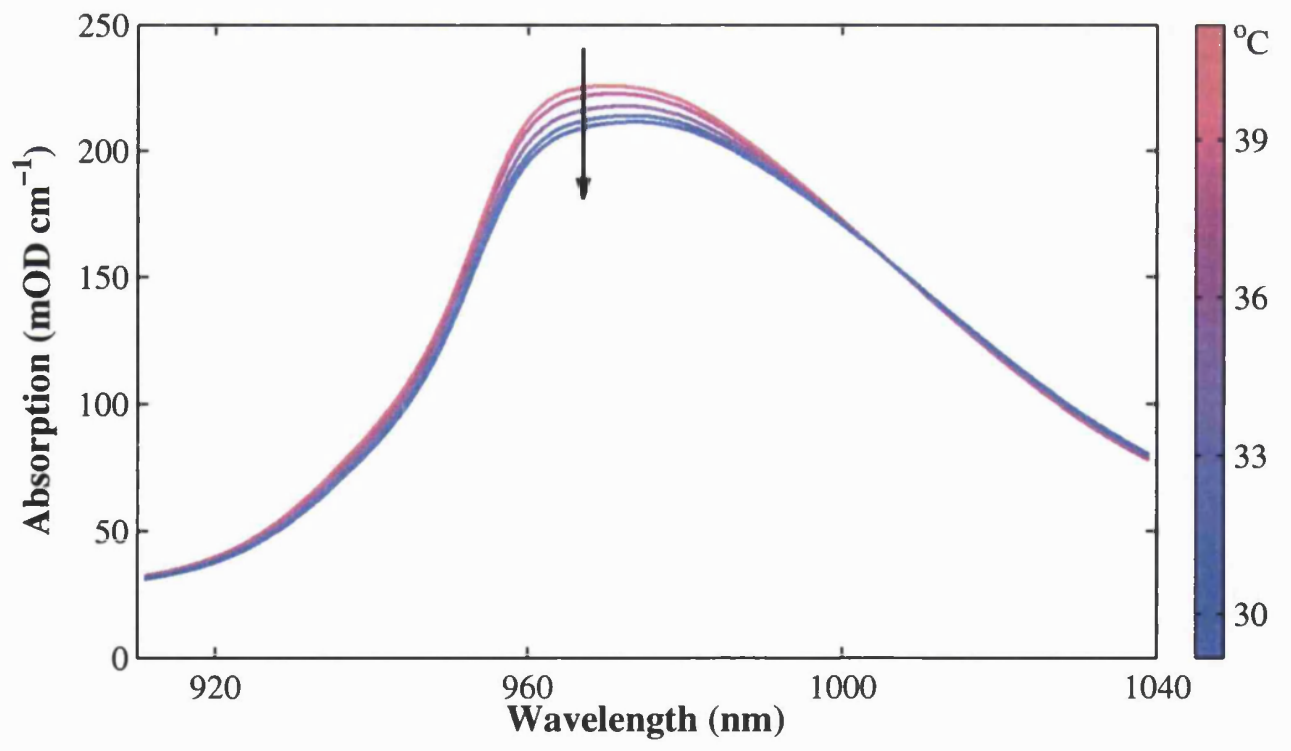

Figure 5.10: NIR absorption coefficient of the $970 \mathrm{~nm}$ water absorption band measured using Method II as a function of temperature between 41 and $29^{\circ} \mathrm{C}$ - arrow indicates direction of decreasing temperature. For clearer display the measured range has been cropped and the spectra have been smoothed with a filter (see text for details). 
The following section will discuss the differences between Methods I and II and the results obtained, including a discussion of the error analysis of the calculated absorption coefficients given in Appendix A.

\subsubsection{Comparison and Further Analysis of Results I and II}

The main differences between Method I and II and the subsequent analysis of the results are listed in Table 5.3. The majority of the difficulties associated with Method I were resolved by Method II. For completeness a comparison of the results obtained by these two methods has been made by determining the temperature coefficient at each wavelength, i.e. the slope of absorption against temperature. These temperature coefficients are shown in Figure 5.11, including those observed by several other groups (Langford et al, 2001; Pegau and Zaneveld, 1994). The differences in the measurements of the ranges 650-805 $\mathrm{nm}$ and $765-920 \mathrm{~nm}$ using Method I are made especially clear in the left-hand graph of

\begin{tabular}{l|l}
\hline Method I & Method II \\
\hline $\begin{array}{l}\text { Reference measurement performed } \\
\text { through } 1 \mathrm{~cm} \text { of water - correction } \\
\text { applied for the change in absorption } \\
\text { by the reference volume with tem- } \\
\text { perature in the calculation of } \mu_{a}\end{array}$ & $\begin{array}{l}\text { Reference measurement performed } \\
\text { through minimum of } 0.12 \mathrm{~cm}-\text { no } \\
\text { correction made, resulting in a per- } \\
\text { centage difference of }< \pm 0.01 \% \text { in } \\
\text { the calculation of } \mu_{a}\end{array}$ \\
\hline $\begin{array}{l}\text { Thin layer of silicone oil added to } \\
\text { reference volume to reduce water } \\
\text { evaporation - absorption due to oil } \\
\text { seen in water absorption spectrum }\end{array}$ & $\begin{array}{l}\text { No silicone oil added }- \text { no interfer- } \\
\text { ence from oil spectrum, evaporation } \\
\text { kept to minimum at }< \pm 0.4 \% \text { loss } \\
\text { in volume }\end{array}$ \\
\hline $\begin{array}{l}\text { Bands positioned around } 740,840 \\
\text { and } 970 \mathrm{~nm} \text { measured separately } \\
\text { with spectral resolution of } 0.62 \mathrm{~nm}- \\
\text { required range matching for further } \\
\text { analysis }\end{array}$ & $\begin{array}{l}\text { Bands at } 740 \text { and } 840 \mathrm{~nm} \text { measured } \\
\text { simultaneously with spectral resolu- } \\
\text { tion of } 1.24 \text { nm }(\text { much smaller than } \\
\text { absorption band widths }- \text { no range } \\
\text { matching required }\end{array}$ \\
\hline $\begin{array}{l}\text { Additional volume for absolute mea- } \\
\text { surements equates to } 12 \text { cm for } \\
\text { ranges } 650-805 \mathrm{~nm} \text { and } 765-920 \mathrm{~nm} \\
\text { and } 1 \mathrm{~cm} \text { for range } 900-1050 \mathrm{~nm}\end{array}$ & $\begin{array}{l}\text { Additional volume equates to } 14 \mathrm{~cm} \\
\text { for range } 600-925 \mathrm{~nm} \text { and } 4 \mathrm{~cm} \text { for } \\
\text { range } 900-1050 \mathrm{~nm}-\text { reduces uncer- } \\
\text { tainty in } \mu_{a} \text { calculation }\end{array}$ \\
\hline
\end{tabular}

Table 5.3: Main differences between Methods I and II for the measurement of the water absorption spectrum 

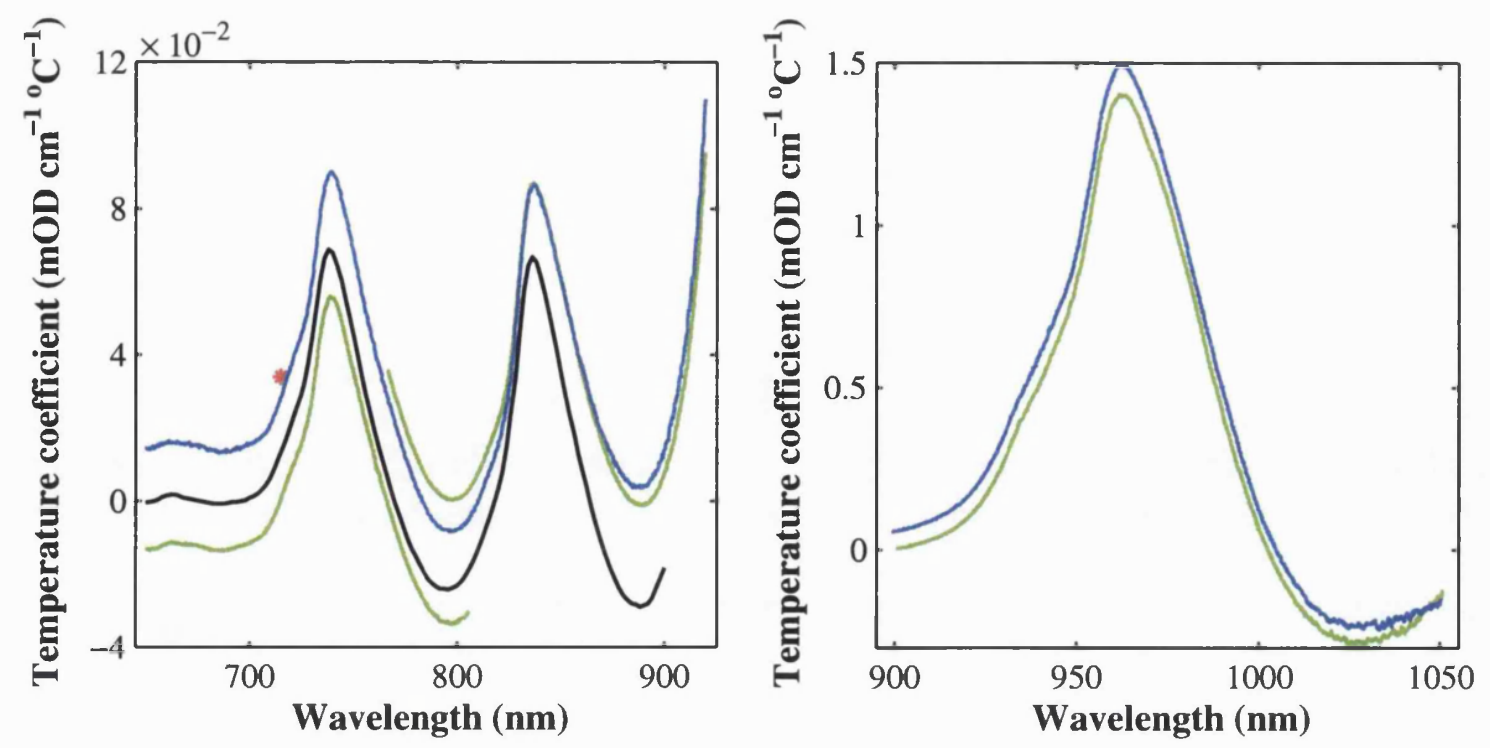

Figure 5.11: Temperature coefficient of the water absorption coefficient as determined by Method I (-), Method II (-), Langford et al (2001) (-) and Pegau and Zaneveld (1994) $(*)$. Left: in the range $650-920 \mathrm{~nm}$. Right: in the range $900-1050 \mathrm{~nm}$.

Figure 5.11, in which their temperature coefficients are seen to be significantly different. This re-enforces the need for measuring simultaneously the two bands at 740 and $840 \mathrm{~nm}$, as was the case for Method II. The temperature coefficients determined from the measurements of Method I and II in the region 770-920 $\mathrm{nm}$ are very similar, supporting the earlier choice of matching the Method I measurements of the $740 \mathrm{~nm}$ band to those of the $840 \mathrm{~nm}$ band, as described in Section 5.1.2.3. The temperature coefficient determined by Pegau and Zaneveld (1994) at $715 \mathrm{~nm}$ agrees well with that determined from the measurements of the extended range using Method II. However, the temperature coefficients determined by Langford et al (2001) in the range 650-900 $\mathrm{nm}$ are significantly different to those of the Method II measurements. In their study (Langford et al, 2001) the authors describe how they subtracted a linear fit to the absorption values in the region $400-500 \mathrm{~nm}$ and the points at 772, 816 and $864 \mathrm{~nm}$, where they believe the temperature coefficient to be zero, in order to remove the effects of instrumental drift during their experiment. They give no reason as to why these particular wavelengths were chosen and offer no evidence to suggest that the temperature coefficient is actually zero at these points. The measurements described here using Methods I and II could certainly be subject to instrumental drift, particularly a drift in the tungsten-halogen light source output with time, which could introduce spurious temperature dependencies into the absorption calculation. No correction was made for instrumental drift in this case, however, since it cannot be simultaneously determined. As a control-type experiment the change in attenuation was monitored over 


\begin{tabular}{lll|ll}
\hline \multicolumn{4}{c}{ Percentage error in $\mu_{a \mathrm{~T}}(\%)$} \\
\hline Method I & \multicolumn{2}{c}{ Method II } \\
\hline $650-805 \mathrm{~nm}$ & $765-920 \mathrm{~nm}$ & $900-1050 \mathrm{~nm}$ & $650-925 \mathrm{~nm}$ & $900-1050 \mathrm{~nm}$ \\
\hline $3.18,2.99$ & $3.01,3.01$ & $3.14,3.67$ & $3.23,3.00$ & $3.00,3.04$ \\
\hline
\end{tabular}

Table 5.4: Percentage errors in $\mu_{a \mathrm{~T}}$ determined for both Method I and II. Values indicate the first and last wavelengths within each measured range.

a period of several hours during stable and varying temperatures within the incubator using the experimental set-up described in Sections 5.1.1 and 5.1.2 (but with no water in the glass tube). From these studies it was not possible to determine a consistent rate of drift in attenuation with time or temperature, but the following observations were made. At a stable temperature the change in attenuation was significant (-5 to $-10 \mathrm{mOD})$ during the first 30-60 minutes of operating the lamp. This is likely to be due to the increase in light output from the bulb as the tungsten filament reaches its maximum operating temperature. After this initial 'warming up' period, the rate of change in attenuation varied between 0 and $\pm 1 \mathrm{mOD}$ per hour. It was observed that this rate appeared to correlate to the age of the bulb, the maximum rates occurring when the bulb was either fairly new (less than 5 hours of use) or relatively old (compared to the manufacturer's estimate of 50 hours for the bulb life-time). No correlation between the rate of change in attenuation and temperature inside the incubator was observed. In an attempt to minimise the potential drift in the light intensity, therefore, the measurements of the water absorption spectrum using Method I and II were made when the bulb was between 10 and 40 hours old, and the lamp was allowed 2 hours 'warm-up' time before the measurements began. It is possible, though, that the amount of drift in the measurement of the $740 \mathrm{~nm}$ band using Method I was significantly greater than for the other measurements, as suggested by Figure 5.11 .

An error analysis for the calculation of the water absorption coefficient measured using Methods I and II based on the uncertainties in the intensity and height measurements has also been performed, the details of which are given in Appendix A. Table 5.4 gives the percentage errors in $\mu_{a \mathrm{~T}}$ at the first and last wavelengths in each range for both methods (where the errors differ with temperature the most extreme value has been quoted). The error in the calculated absorption coefficient determined by both methods is dominated by the error in the additional height due to the $\pm 3 \%$ error in the volume delivery of the syringe driver. In the region $720-900 \mathrm{~nm}$ the error in $h_{2} \mathrm{~T}_{S}$ (Equation (A.11)) contributes over $99 \%$ to the total error in $\mu_{a \mathrm{~T}}$ for both Method I and II. At wavelengths shorter than 
$720 \mathrm{~nm}$ the contribution to the error in $\mu_{a \mathrm{~T}}$ by the intensity measurements becomes more significant, since the intensity in this region is low and as a result its percentage error is greater. At $650 \mathrm{~nm}$ the percentage error in $\mu_{a \mathrm{~T}}$ for Method II slightly exceeds that for Method I (by a maximum of $\sim 0.1 \%$ ) due to the fact that the intensity measurements in Method II were averaged over less data points than in Method I (see Tables 5.1 and 5.2 and Sections A.1 and A.2). In the region $720-900 \mathrm{~nm}$ the percentage errors in $\mu_{a \mathrm{~T}}$ are very similar for both methods, whereas in the range 900-1050 nm the error in $\mu_{a \mathrm{~T}}$ is less for Method II, due to the addition of a greater volume of water in Method II. In conclusion, therefore, the improvements of Method II do not adversely affect the accuracy of the calculation of the water absorption coefficient, and in some cases improve it.

\subsection{Effects of Water Binding on the NIR Absorption Spec- trum}

As discussed in Section 2.1.1, water in the body exists as both free and bound water, the percentage of bound water depending on the tissue type (i.e. its constituents). The binding of water to the extent that occurs in the body may affect its NIR absorption spectrum, which in turn will affect any algorithm based on the magnitude and wavelength-dependence of water absorption in tissue, such as the one being developed in this project. This section therefore looks at the effect on the NIR water spectrum of water binding to different solutes. As discussed in Section 2.1.1, water binding to other molecules tends to affect the spectrum by increasing the strongly hydrogen-bonded component of water. Section 4.3.1.1 described how stronger hydrogen bonding (whether due to temperature effects or a more favourable binding to another molecule) affects the fundamental $\mathrm{O}-\mathrm{H}$ stretching band by shifting it to longer wavelengths (lower vibrational frequencies). This phenomenon is observed especially clearly in the $970 \mathrm{~nm}$ water absorption band (a combination of the fundamental stretch) with decreasing temperature (e.g. Figure 5.10). For the same temperature, therefore, one might expect to see the position of the NIR water absorption bands red-shifting with increasing water binding.

\subsubsection{Organic Solute: Sodium Carboxymethylcellulose}

The NIR water absorption spectrum was re-measured on addition of different concentrations of sodium carboxymethylcellulose (Blanose ${ }^{\circledR}$ cellulose gum 7HF, Hercules-Aqualon, Widnes UK), known as CMC. Blanose ${ }^{\circledR}$ CMC is used predominantly as a thickening 
agent in a wide range of industries, including pharmaceuticals, food and cosmetics. It has a high water-binding capacity and when dissolved in water produces a clear viscous gel-like solution. At $25^{\circ} \mathrm{C}$ the viscosity of a $1 \%$ solution of $7 \mathrm{HF} \mathrm{CMC}$ is reported to be in the range 1500-2500 $\mathrm{mPa} \cdot \mathrm{s}$, compared with $\sim 0.9 \mathrm{mPa} \cdot \mathrm{s}$ for pure water. Unfortunately the precise extent of the water binding could not be ascertained for a given concentration of CMC dissolved in water, although it is known that this will depend on the availability of polar sites within the cellulose-based molecule capable of hydrogen-bonding to water molecules.

\subsubsection{Experimental Method}

The CMC solution was prepared by dissolving its powdered form in distilled water at three different concentrations: $0.1,0.2$ and $0.3 \%$. All three solutions were clear with a thicker consistency than water but still fluid enough to be easily poured. Using the set-up described in Section 5.1.1 the absorption coefficients of the three solutions were measured in the ranges $595-920 \mathrm{~nm}$ and $900-1050 \mathrm{~nm}$. The slit width, grating and spectral resolution for the two ranges were the same as those reported for the measurement of the water absorption spectrum using Method II (Section 5.1.2.3). The spectra were measured at a single temperature only, the ambient temperature inside the closed unheated incubator, approximately $31^{\circ} \mathrm{C}$. As for Method I of the water absorption measurements (Section 5.1.2.1), a reference measurement through $1 \mathrm{~cm}$ of the solution was made, then an additional volume was added and an absolute measurement performed. The additional heights of the solutions for the absolute measurements were 13 and $3 \mathrm{~cm}$ for the $595-920 \mathrm{~nm}$ and 900-1050 nm ranges respectively. For each concentration 100 spectra were acquired and averaged together for the reference and absolute intensity measurements, using an acquisition time of 500 and $800 \mathrm{~ms}$ for the $595-920 \mathrm{~nm}$ and $900-1050 \mathrm{~nm}$ ranges respectively.

\subsubsection{Results and Discussion}

The absorption coefficients for the three solutions could be calculated using Equation (5.13), since the temperature of the water during the reference and the single absolute measurement were the same to within $\pm 0.2^{\circ} \mathrm{C}$. This equation is the same as the Method I definition of $\mu_{a \mathrm{~T}}$ in Equation (5.21) with temperature $\mathrm{T}$ equal to $\mathrm{T}_{r}$. As before the standardising temperature was $37^{\circ} \mathrm{C}$.

Figure 5.12 shows the absorption coefficients of the three solutions of CMC in the range 


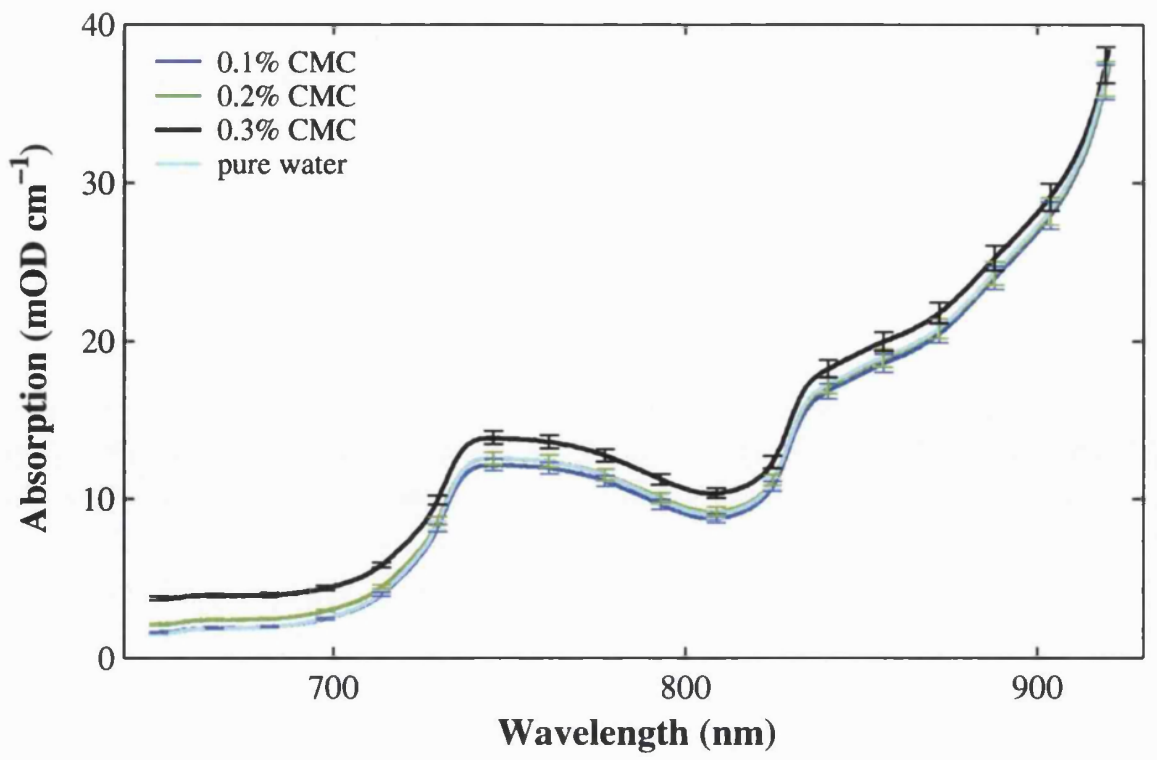

Figure 5.12: Absorption coefficient of CMC solutions at concentrations 0.1, 0.2 and $0.3 \%$ (at a mean temperature of $30.7^{\circ} \mathrm{C}$ ) in the range 650-920 $\mathrm{nm}$ compared to pure water. The error bars on the absorption spectra were calculated in Appendix A.

$650-920 \mathrm{~nm}$, including the pure water absorption spectrum in this range as measured using Method II (Section 5.1.2.3). The mean temperature at which the CMC spectra were measured is $30.7^{\circ} \mathrm{C}\left( \pm 0.2^{\circ} \mathrm{C}\right)$, and the pure water spectrum displayed was measured at the same temperature. Figure 5.13 shows the absorption coefficients of the CMC solutions in the range 900-1050 $\mathrm{nm}$. The mean temperature at which the spectra were measured is $31^{\circ} \mathrm{C}\left( \pm 0.7^{\circ} \mathrm{C}\right)$, and the pure water absorption spectrum displayed (measured using

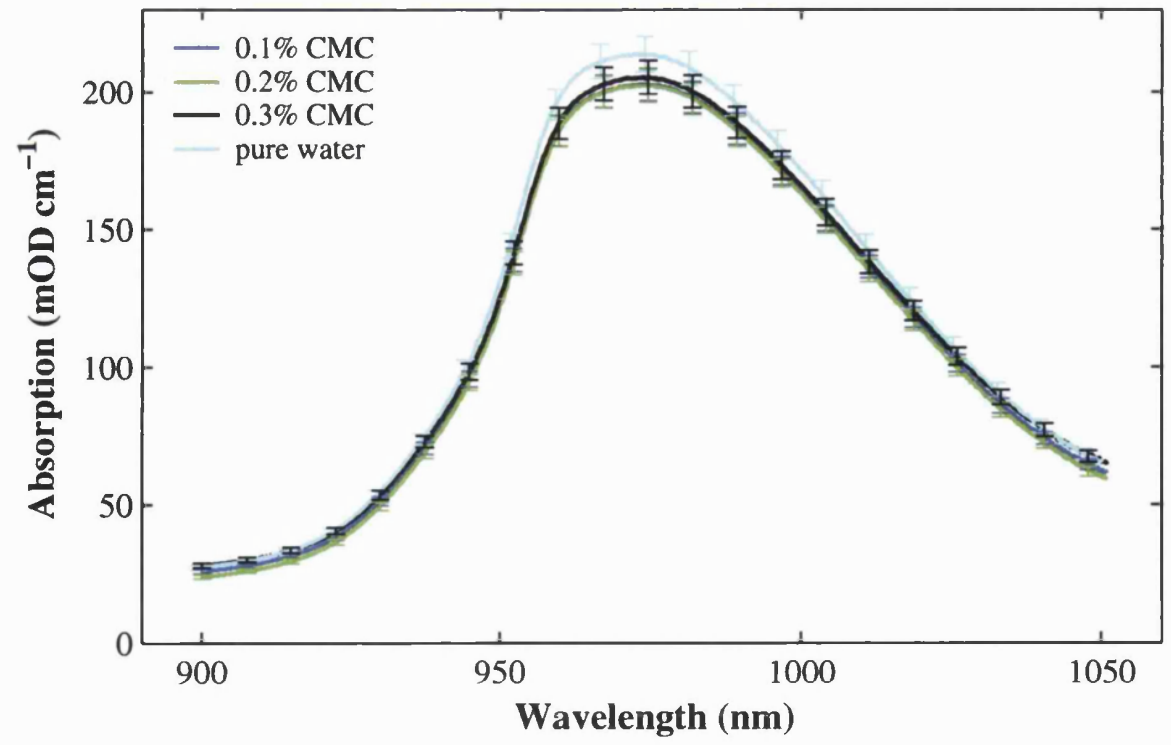

Figure 5.13: Absorption coefficient of CMC solutions at concentrations $0.1,0.2$ and $0.3 \%$ (at a mean temperature of $31.0^{\circ} \mathrm{C}$ ) in the range 900-1050 nm compared to pure water. The error bars on the absorption spectra were calculated in Appendix A. 
Method II) was also measured at this temperature.

In Figure 5.12 the absorption spectra of the three solutions of $\mathrm{CMC}$ become significantly different from one another below approximately $850 \mathrm{~nm}$, as shown by the fact that their error bars (calculated in Appendix A) no longer overlap. In particular the $0.3 \%$ solution differs from the other two below this wavelength, and the 0.2 and $0.1 \%$ solutions differ below about $720 \mathrm{~nm}$. Compared to the pure water spectrum, the $0.1 \%$ solution of CMC does not differ significantly in its absorption spectrum over the whole range, the $0.2 \%$ solution only differs from water below $720 \mathrm{~nm}$ and the $0.3 \%$ solution differs below approximately $840 \mathrm{~nm}$. Figure 5.13 shows that the absorption spectra of the three CMC solutions do not differ significantly except below approximately $930 \mathrm{~nm}$. In comparison to pure water, only the $0.2 \%$ solution absorption spectrum differs significantly from the water spectrum, and then only at the tail ends of the absorption band. It is possible that the small differences in the absorption spectra of the three CMC solutions are due to differences in the temperature at which they were measured, and likewise for the difference between the CMC absorption spectra and the pure water spectrum. However, correcting the pure water spectrum to the temperature at which each of the CMC spectra were measured using the temperature coefficients determined previously for pure water (Figure 5.11) did not greatly improve the agreement between the spectra, and in some cases degraded it. (It should be noted though that no account is made for the uncertainty of the temperature measurement). It is also possible that overtones of the fundamental C-H stretching mode could appear in the regions measured which could affect the shape of the measured bands.

From these measurements it is not clear whether or not the binding of water molecules to the cellulose polymers is affecting the water absorption spectrum. Subtle differences in spectral shape which could indicate the effect of water binding are probably best examined in the second derivative (see Section 7.1). Comparing the absorption spectra of the three solutions of $\mathrm{CMC}$ in the second derivative revealed no differences with $\mathrm{CMC}$ concentration and no differences relative to the water spectrum at the same temperature. Thus the experiments described in this section do not rule out the possible effect of binding to organic molecules on the NIR water spectrum, but it was not possible to observe at the extent of water binding present (although how this relates to that in biological tissues is unknown). 


\subsubsection{Inorganic Solute: Sodium Chloride}

The NIR absorption spectrum of a $0.9 \%$ solution of sodium chloride (saline) was measured to determine the effects of water binding to ions present in the body. At $0.9 \%$ the saline solution is isotonic, i.e. it exerts the same osmotic pressure as the fluid in the intracellular compartment. When a salt dissolves in water it disassociates into its constituent ions. This is because it is more energetically favourable for water molecules to form clusters around the ions, breaking intermolecular hydrogen bonds in order to bind to the ions instead. In the case of saline the $\mathrm{O}^{\delta-}$ site of the water molecule hydrogen bonds to an $\mathrm{Na}^{+}$ion and the $\mathrm{O}-\mathrm{H}^{\delta+}$ site hydrogen bonds to a $\mathrm{Cl}^{-}$ion. Each ion can bind approximately six water molecules, so for each pair of ions six intermolecular hydrogen bonds are broken. In addition to comparing the absolute measurements at a given temperature, the temperature dependence of the saline absorption spectrum was measured, to determine if this was significantly different to that of pure water.

\subsubsection{Experimental Method}

The absorption spectrum of isotonic saline was measured in the ranges 600-925 and 900-1050 nm between 40 and $30^{\circ} \mathrm{C}$ using Method II described in Section 5.1.2.3. The acquisition parameters for the two ranges were the same as those for the measurements of the pure water spectrum as a function of temperature, given in Table 5.2. The rate of evaporation of water from a vessel decreases with increasing salinity as the vapour pressure of the liquid decreases. Over the 3.5 hour period of the experiment the amount of saline evaporation from the equivalent measuring cylinder was less than $0.2 \%$ of the total volume compared to $0.4 \%$ with pure water (see Section 5.1.2.3).

\subsubsection{Results and Discussion}

As for the pure water measurements described in Section 5.1 the saline spectra were averaged such that the maximum temperature change between spectra was $0.05^{\circ} \mathrm{C}$. The absorption coefficient as a function of temperature was calculated using Equation (5.22) given in Section 5.1.2.4 (with $\mathrm{T}_{S}=37^{\circ} \mathrm{C}$ ). As an initial comparison between the absorption coefficients of pure water and the $0.9 \%$ saline solution, Figure 5.14 shows the saline and water absorption spectra at $30^{\circ} \mathrm{C}$ in the ranges $650-925$ and $900-1050 \mathrm{~nm}$. The calculation of the error bars on the absorption coefficients is presented in Appendix A. The absorption coefficients for pure water and the saline solution lie within each oth- 

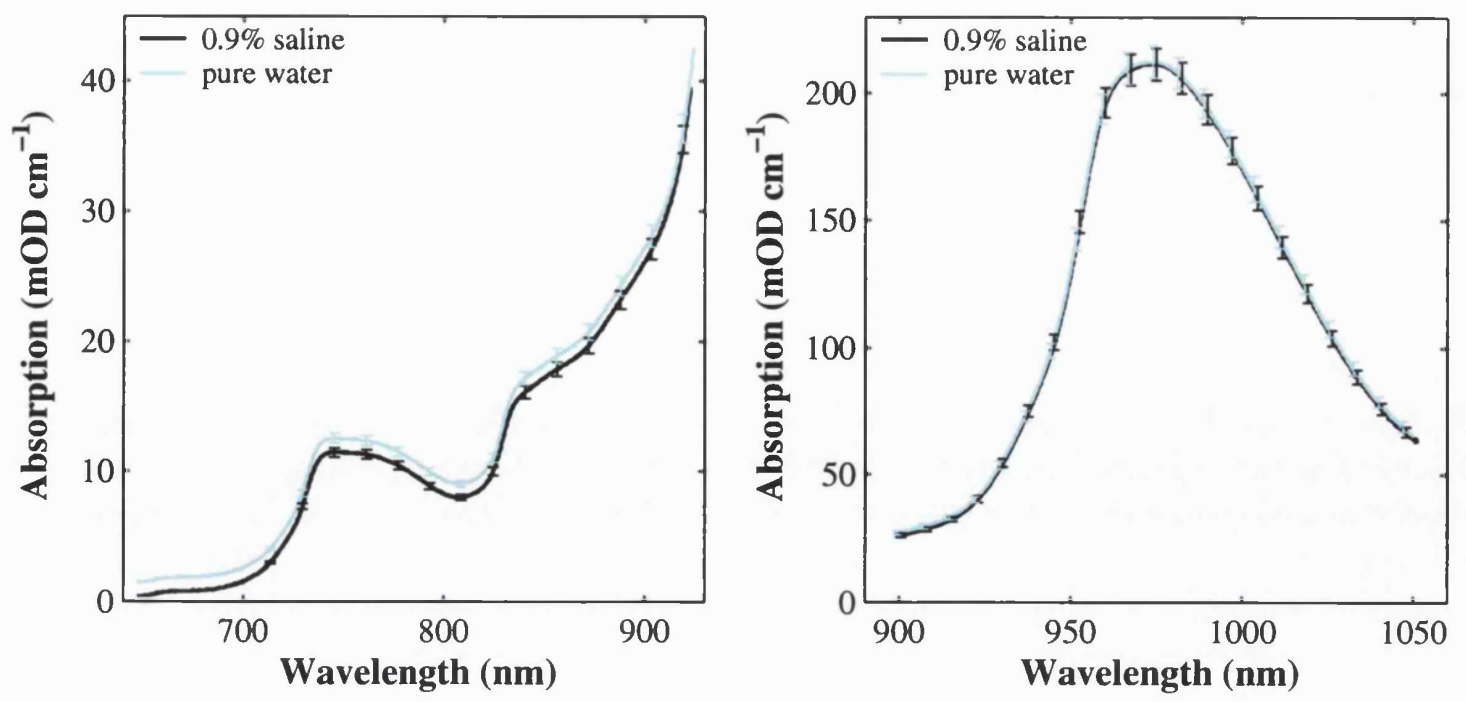

Figure 5.14: Absorption coefficient of isotonic saline compared to pure water at $30^{\circ} \mathrm{C}$ in the ranges $650-925 \mathrm{~nm}$ (left) and 900-1050 nm (right). The error bars on the absorption spectra were calculated in Appendix A.

ers error bars from approximately $840-1050 \mathrm{~nm}$, with particularly good agreement in the range covering the $970 \mathrm{~nm}$ absorption band. Below $840 \mathrm{~nm}$ the absorption spectra are significantly different. Sodium chloride has no absorption bands of its own in the near or mid-infrared (Hirschfield, 1985), so any differences between the absorption spectrum of saline and that of pure water (at a given temperature) should alone be due to the effect on the water absorption bands of a perturbation of intermolecular hydrogen bonding.

Pegau et al (1997) studied the effect of salinity $(\mathrm{NaCl})$ in the range $0-3.8 \%$ on the absorption spectra of pure water. At 715 and $750 \mathrm{~nm}$ they determined salinity coefficients of -0.027 and $0.064 \mathrm{mOD} \mathrm{cm}^{-1}(\% \mathrm{NaCl})^{-1}$ respectively at a (mean) temperature of $21^{\circ} \mathrm{C}$. This equates to changes in absorption for a $0.9 \%$ saline solution relative to pure water of -0.024 and $0.058 \mathrm{mOD} \mathrm{cm}^{-1}$ at 715 and $750 \mathrm{~nm}$ respectively. These very small changes with salinity do not account for the differences observed between the water and saline absorption spectra in Figure 5.14 (left hand graph). These differences are not observed in the second derivative (or in fact the first derivative), indicating that they could be due to the significant effects of instrumental drift in the region where absorption is the lowest.

The absorption spectra of the saline solution measured between 40 and $30^{\circ} \mathrm{C}$ do not appear visibly different from those of pure water (Figures 5.9 and 5.10) so will not be displayed again. Figure 5.15 compares the temperature coefficients of the saline absorption spectra to the pure water spectra. The temperature coefficients of saline and water appear very similar (error bars have not been calculated for these coefficients) with the the greatest differences below approximately $830 \mathrm{~nm}$, particularly where the temperature coefficient is 

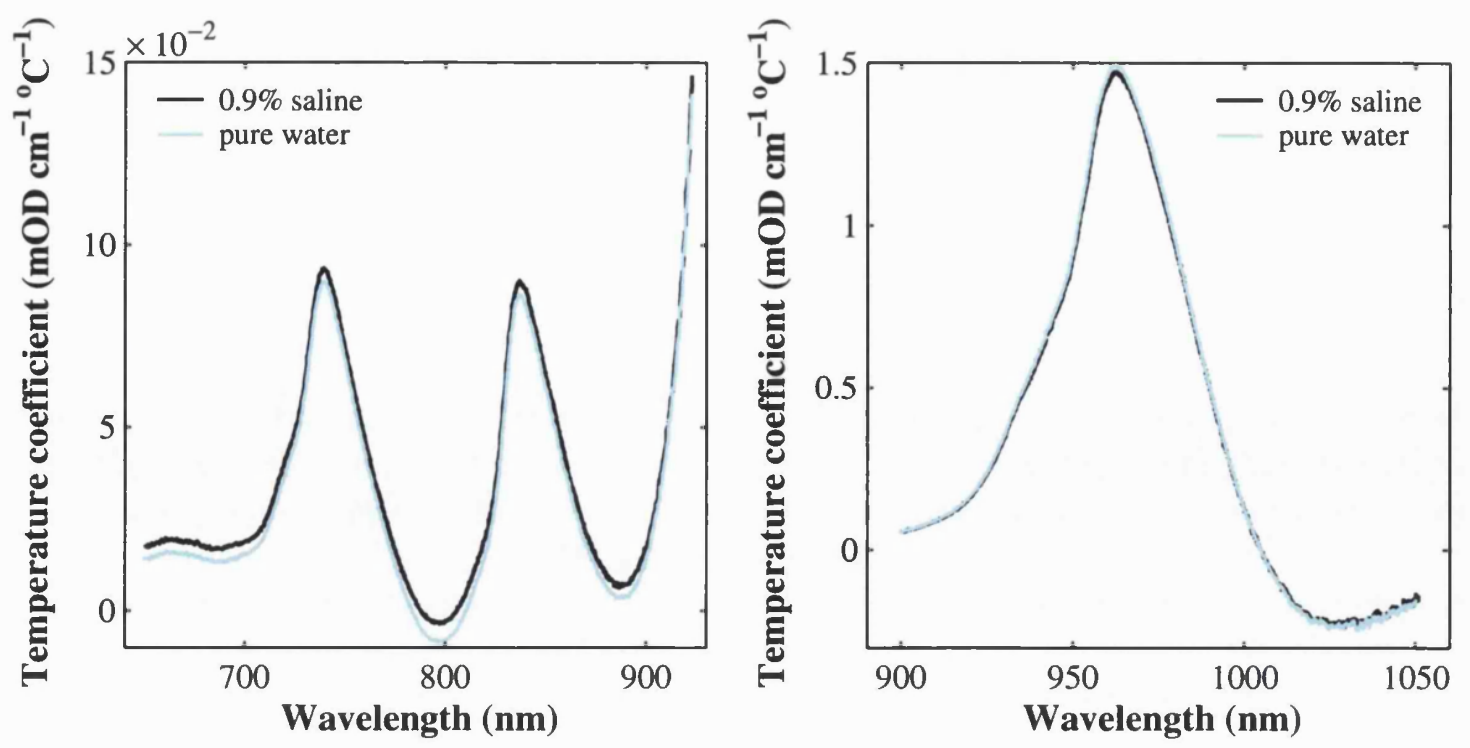

Figure 5.15: Temperature coefficients of isotonic saline and pure water absorption spectra in the ranges $650-925$ and $900-1050 \mathrm{~nm}$, measured between 40 and $30^{\circ} \mathrm{C}$.

close to zero. In their study Pegau et al report no significant differences between the temperature coefficients of saline and pure water over a range of wavelengths in the visible and NIR.

The measurements described in this section (Section 5.2) have demonstrated little evidence of the effect of water binding on the NIR absorption spectrum of water or its temperature-dependence. The differences between the measured absorption spectra and that of pure water are most significant in the low absorption region of the spectrum. This suggests that the dominant cause of these differences is instrumental drift, since the effect of water binding should be more obvious in the region of greater absorption around the $970 \mathrm{~nm}$ absorption band, as is the case with temperature (see Figures 5.11 and 5.15).

\subsection{Temperature Calibration of the NIR Water Absorption Spectrum}

Having accurately measured the temperature response of the NIR absorption spectrum of water (Section 5.1), this section will discuss various methods of calibrating the water spectrum against the measured temperature. The aim is to find a parameter (or parameters) of the water absorption spectrum that can be directly related to temperature, and to describe the relationship between the temperature-dependent parameters and the absorption spectrum in such a way that it can ultimately be employed in a tissue temperature prediction 
algorithm. All but the first method are known as multivariate calibration techniques since they use more than one 'independent' variable (e.g. absorption at several wavelengths) to calibrate the 'dependent' variable (e.g. temperature) by means of multiple linear regression (MLR) methods. These techniques are widely used in the field of chemometrics to relate chemical or physical properties to a measured response, e.g. analyte concentrations to spectroscopic measurements. An excellent general reference to multivariate calibration in a spectroscopic context is provided by Martens and Næs (1991). All but the final two methods are applied only to the water absorption spectra measured using Method I.

\subsubsection{Gaussian Component Method}

The first temperature calibration method to be described involves the deconvolution of the water absorption bands into a set of Gaussian functions that vary in width and amplitude with temperature. The justification behind this approach is based on the mixture model of water, in which liquid water is said to consist of a number of different molecular species of varying degrees of polymerisation by hydrogen-bonding, in thermal equilibrium with one another (see Section 4.3.1.1). Hence this method attempts to quantify the spectral shifts with temperature by deconvolving the absorption bands into their component species and describing their temperature-dependence analytically. As discussed in Section 4.3.1.1, various authors have used this method in order to characterise the temperature-dependence of the water absorption spectrum (Del Bianco et al, 2000; Pegau et al, 1997; Walrafen, 1967), although not to predict temperature in unknown samples.

The water absorption spectrum $S$ as a function of temperature T and wavelength $\lambda$ can be described as a superposition of $i$ Gaussian components as follows

$$
S_{(\mathrm{T}, \lambda)}=\sum_{i} G_{i(\mathrm{~T}, \lambda)}
$$

where $G_{i}$ is the value of the $i$ th Gaussian component at wavelength $\lambda$, given by

$$
G_{i(\mathrm{~T}, \lambda)}=a_{i(\mathrm{~T})} \exp \left(-\left(\frac{\lambda-\lambda_{i}}{w_{i(\mathrm{~T})}}\right)^{2}\right)
$$

where $\lambda_{i}$ is the fixed peak position of the $i$ th component and $a_{i(\mathrm{~T})}$ and $w_{i(\mathrm{~T})}$ are the amplitude and width of the $i$ th component at temperature $T$ respectively. The amplitude 
and width of the components can then be approximated as linear functions of temperature

$$
\begin{aligned}
& a_{i(\mathrm{~T})}=a_{0 i}+a_{1 i} \mathrm{~T} \\
& w_{i(\mathrm{~T})}=w_{0 i}+w_{1 i} \mathrm{~T}
\end{aligned}
$$

where $a_{0 i}$ and $a_{1 i}$ and $w_{0 i}$ and $w_{1 i}$ are the offsets and slopes for the regression of the amplitude and width against temperature respectively.

The water absorption spectra measured using Method I (Section 5.1.2.1) were matched together over the entire wavelength range $650-1050 \mathrm{~nm}$ at six different temperatures between 42 and $32^{\circ} \mathrm{C}$. Gaussian functions were fitted to the spectrum at the maximum temperature of $42^{\circ} \mathrm{C}$ using a peak-fitting tool in the spectral acquisition software. Starting with a component positioned at $970 \mathrm{~nm}$ to account for the largest absorption band, the number of components included in the fit was incremented progressively, the approximate position of the next component chosen according to the (absolute) maximum of the previous fit residuals. For the fitting of the absorption spectrum at the maximum temperature the peak position, amplitude and width of the Gaussian components were not restrained, such that the best component parameters minimised the fit residuals. A total of twelve Gaussian components were thus fitted to the spectrum at the maximum temperature. An unrestrained baseline offset was also included (approximately $1.4 \mathrm{mOD}$ ) which further improved the fit residuals. The fit was then repeated for the other five temperatures, allowing the amplitude and the width of the components to vary with temperature but fixing their peak wavelength positions. Figure 5.16 shows the twelve Gaussian components, the total fit and the measured absorption spectrum in the range $650-1050 \mathrm{~nm}$ at $42^{\circ} \mathrm{C}$.

A linear least-squares regression of the widths and amplitudes of each of the components against temperature was then performed. The coefficients of the fits, i.e. the offsets and slopes given by the $a$ and $w$ coefficients in Equation (5.26), are listed in Table 5.5 for each component. The coefficients $r_{a}^{2}$ and $r_{w}^{2}$ for the correlation between the amplitudes and widths and temperature are also given. Their predominantly high values indicate that the assumption of a linear relationship between temperature and the widths and amplitudes of the Gaussian components is reasonable within this temperature range. The few exceptions for which the $r^{2}$ coefficients are low are due to the extremely small variation in the particular parameter with temperature. The coefficients listed in Table 5.5 were then used to generate the modelled water absorption spectra $S$ at the various temperatures using Equations (5.24)-(5.26). An estimate of the 'goodness of fit' of the Gaussian model 


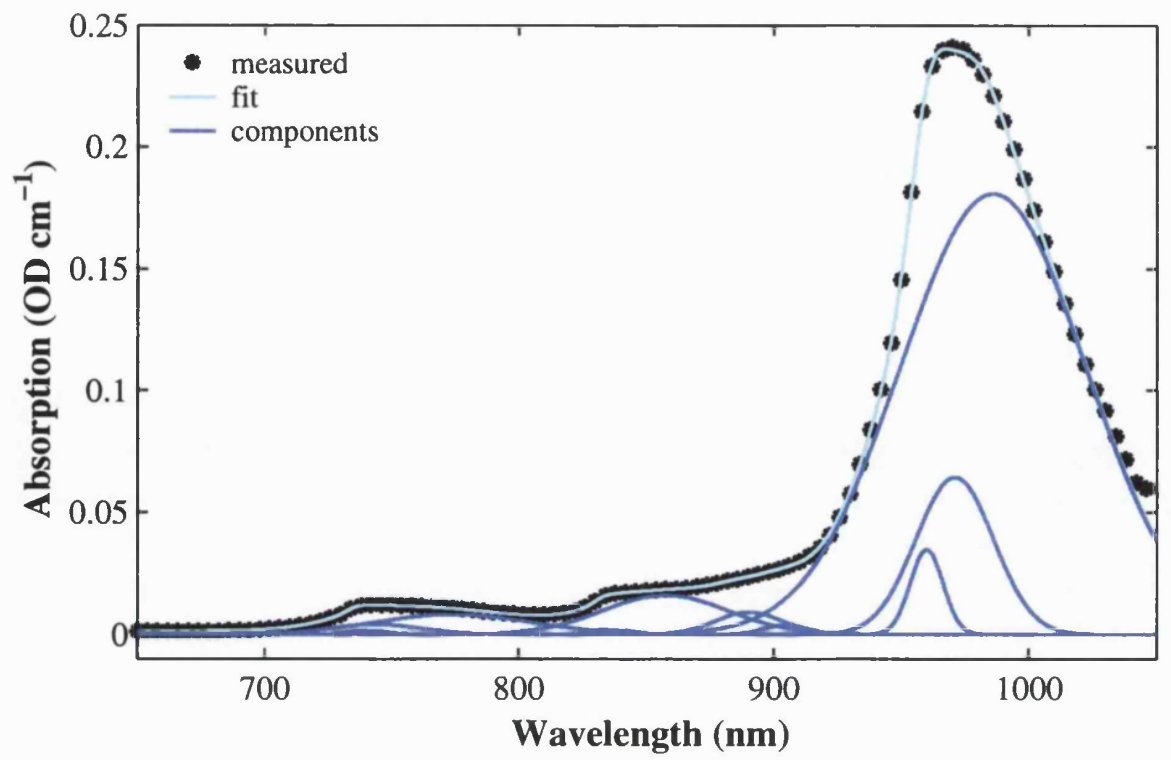

Figure 5.16: Decomposition of the NIR water absorption spectrum measured (using Method I) at $42^{\circ} \mathrm{C}$ in the range $650-1050 \mathrm{~nm}$ into 12 Gaussian components. The horizontal line close to zero is the $1.4 \mathrm{mOD}$ offset, included to improve the fit.

can be obtained by calculating the fit residuals $E$ as follows

$$
E_{(\mathrm{T}, \lambda)}=S_{(\mathrm{T}, \lambda)}-\mu_{a(\mathrm{~T}, \lambda)}
$$

where $\mu_{a(\mathrm{~T}, \lambda)}$ is the experimentally determined absorption spectrum. At the minimum temperature, for which the poorest fit was obtained, the residuals are approximately \pm 0.2 $\mathrm{mOD}$ or less in the range $650-920 \mathrm{~nm}$, i.e. within the error determined for the experimentally measured $\mu_{a}$ values in this region (see Section 5.1.3). The residuals increase to a maximum of $\pm 3 \mathrm{mOD}$ in the range $920-1030 \mathrm{~nm}$, still within the error on the experimentally determined $\mu_{a}$ values. Above $1030 \mathrm{~nm}$ the residuals increase to an absolute value of approximately $10 \mathrm{mOD}$, greater than the error on the measured $\mu_{a}$ values. As noted by Del Bianco et al (2000), the type of baseline correction included in the fit can greatly affect the peak position of the Gaussian components, and hence the goodness-of-fit. It is possible, therefore, that the constant baseline subtraction performed here is not appropriate for the region above $1030 \mathrm{~nm}$ and that a higher order correction may be more suitable. The temperature of a set of 'unknown' absorption spectra could be predicted by fitting Gaussians at the peak positions found during this calibration and using the known temperature-dependence of the widths and amplitudes of the twelve components. From various investigations, however, it is apparent that the calibration is extremely sensitive to the baseline correction and fit range employed. For this reason, a validation of the 


\begin{tabular}{lllllll}
\hline $\begin{array}{l}\text { Position } \\
(\mathrm{nm})\end{array}$ & $\begin{array}{l}a_{0} \\
(\mathrm{mOD})\end{array}$ & $\begin{array}{l}a_{1} \\
\left(\mathrm{mOD}^{\circ} \mathrm{C}^{-1}\right)\end{array}$ & $r_{a}^{2}$ & $\begin{array}{l}w_{0} \\
(\mathrm{~nm})\end{array}$ & $\begin{array}{l}w_{1} \\
\left(\mathrm{~nm}^{\circ} \mathrm{C}^{-1}\right)\end{array}$ & $r_{w}^{2}$ \\
\hline 986 & 208.76 & -0.656 & 0.9875 & 49.40 & 0.047 & 0.9613 \\
971 & -4.74 & 1.642 & 0.9997 & 12.31 & 0.220 & 0.9963 \\
960 & 19.68 & 0.359 & 0.9996 & 7.32 & 0.041 & 0.9982 \\
858 & 14.89 & 0.029 & 0.9213 & 32.42 & 0.057 & 0.8265 \\
774 & 8.78 & -0.012 & 0.7833 & 45.20 & -0.040 & 0.2994 \\
890 & 12.57 & -0.079 & 0.9736 & 17.37 & 0.024 & 0.5773 \\
744 & 2.37 & 0.042 & 0.9905 & 17.01 & 0.202 & 0.9978 \\
840 & -0.22 & 0.046 & 0.9961 & 2.80 & 0.143 & 0.9933 \\
741 & -0.53 & 0.048 & 0.9987 & 5.70 & 0.096 & 0.9910 \\
905 & 3.35 & 0.009 & 0.5986 & 5.73 & 0.141 & 0.8849 \\
833 & 1.56 & 0.011 & 0.8980 & 5.58 & 0.010 & 0.5509 \\
736 & 1.00 & 0.004 & 0.8651 & 6.52 & -0.016 & 0.6399 \\
\hline
\end{tabular}

Table 5.5: Coefficients obtained from the linear regression of the amplitudes and widths of the 12 Gaussian components against water temperature

method was not performed on a separate data set. One possible solution to the problem of the baseline offset would be to calibrate against the first or second derivatives of the water spectra. As will be discussed in greater detail in Section 7.1, differentiating the data removes linear backgrounds that may vary between data sets (e.g. due to instrumental drift), which would avoid the problem of including them in the Gaussian fitting.

In some respects, therefore, it would seem that this approach is not very robust and rather empirical. However, some physical meaning underlying the method can tentatively be drawn from the information in Table 5.5. Consider that each of the three measured absorption bands is comprised of four Gaussian components in which each component, as proposed by the mixture model (Section 4.3.1.1), represents a species of water molecule with a certain number of hydrogen bonds. From the results of the Gaussian fitting presented in Table 5.5 one could assign the components at 986, 971, 960 and $905 \mathrm{~nm}$ to the $970 \mathrm{~nm}$ water band, those at $890,858,840$ and $833 \mathrm{~nm}$ to the $840 \mathrm{~nm}$ band, and the components at 774, 744, 741 and $736 \mathrm{~nm}$ to the $740 \mathrm{~nm}$ band. It can be seen from Table 5.5 that the longest wavelength components of the three groups have negative amplitude slopes, that is, their amplitudes increase with decreasing temperature. Regardless of the number of species that supporters of the mixture model of water claim exist, it is generally accepted that as the temperature of water is decreased the strongly hydrogen-bonded component will increase in amplitude at the expense of one or more of the weaker-bonded (or 'free') species (Karmakar and Joarder, 1994; Walrafen, 1967). Moreover, the component 
belonging to the stronger-bonded species may well have a longer peak wavelength than the others, since hydrogen bonding decreases the frequency of the $\mathrm{O}-\mathrm{H}$ vibrational stretch (Pimental and McClellan, 1960). This last observation is fairly speculative, however, since the three absorption bands at 740,840 and $970 \mathrm{~nm}$ are combination bands arising from both bending and stretching vibrations. On the other hand, the amplitudes of all three bands show an overall positive correlation with temperature (Figures 5.3-5.5), as do the fundamental stretching bands (Eisenberg and Kauzmann, 1969). It is possible, therefore, that the peak positions of the different species are predominantly governed by the response of the stretching vibrations to hydrogen-bonding.

\subsubsection{Classical Least-Squares}

The next calibration method to be described is the first of the multivariate techniques: classical least-squares (CLS). The CLS technique is commonly applied to the spectral analysis of multi-component samples in order to calibrate component concentrations (Haaland et al, 1985). The basic assumption is that absorption is linearly related to the component concentrations, as stated by Beer's law in Equation (2.5). In the application of CLS to the temperature calibration of spectroscopic measurements, therefore, the underlying assumption is that absorption is a linear function of temperature at all wavelengths.

The calibration of the water absorption spectra for temperature can be described using matrices as follows

$$
\mathbf{A}=\mathbf{t} \cdot \mathbf{p}+\mathbf{E}_{\mathbf{A}}
$$

where $\mathbf{A}$ is an $(\mathrm{n} \times \mathrm{m})$ matrix of the measured absorption spectra, known as the calibration data set, with $n$ spectra at $m$ wavelengths, $t$ is the $(n \times 1)$ vector of measured temperatures corresponding to each spectrum, $\mathbf{p}$ is $(1 \times \mathrm{m})$ vector of coefficients relating the absorption spectra in $\mathbf{A}$ to the temperatures in $\mathbf{t}$ and $\mathbf{E}_{\mathbf{A}}$ is the $(\mathrm{n} \times \mathrm{m})$ absorption residuals matrix, i.e. the errors not fit by the model. (Hereafter matrices will be displayed as bold capital letters, column vectors as bold lower case letters, scalars as non-bold lower case letters and the transpose of a vector $\mathbf{x}$ (or matrix $\mathbf{X}$ ) will be indicated as $\mathbf{x}^{\prime}$ (or $\mathbf{X}^{\prime}$ ) as is the common convention (Geladi and Kowalski, 1986; Haaland and Thomas, 1988)). Since both $\mathbf{A}$ and $\mathbf{t}$ are known the $\mathbf{p}$ vector can be determined using matrix algebra as follows

$$
\hat{\mathbf{p}}=\left(\mathbf{t}^{\prime} \cdot \mathbf{t}\right)^{-1} \cdot \mathbf{t}^{\prime} \cdot \mathbf{A}
$$

where the caret over the $\mathbf{p}$ indicates that the solution is in the least-squares sense. A 
full derivation of minimisation by the least-squares estimator using matrix manipulation is given elsewhere (Press et al, 1992). For an 'unknown' sample, the calibration-predicted temperature is then

$$
\hat{\mathrm{t}}=\mathbf{a} \cdot \hat{\mathbf{p}}^{\prime} \cdot\left(\hat{\mathbf{p}} \cdot \hat{\mathbf{p}}^{\prime}\right)^{-1}
$$

where $\hat{\mathbf{a}}$ is the $(1 \times \mathrm{m})$ row vector from matrix $\mathbf{A}$ representing the spectrum of the given sample.

The water absorption spectra measured over the ranges $650-805,765-920$ and $900-$ $1050 \mathrm{~nm}$ using Method I were calibrated against temperature using the method described above. Prior to calibration the mean spectrum in each data set $\mathbf{A}$ was subtracted from each of the spectra and the mean temperature was subtracted from the measured values in $\mathbf{t}$. This pre-treatment of the data, known as mean-centering, removes the need to fit a non-zero intercept and is often used in multivariate analyses such as CLS (Haaland and Thomas, 1988).

Figure 5.17 shows the measured versus the calibration estimated temperature for the three ranges. The accuracy of the temperature prediction will depend on the both the validity of the model and the amount of noise on the raw data. Even if the model is correct in the assumption that absorption at all wavelengths varies linearly with temperature, the errors will be greater at wavelengths where the variation in absorption is comparable to the uncertainty on the absorption values. The uncertainties in the temperature prediction will therefore depend on the spectral range employed. This is indicated more clearly in

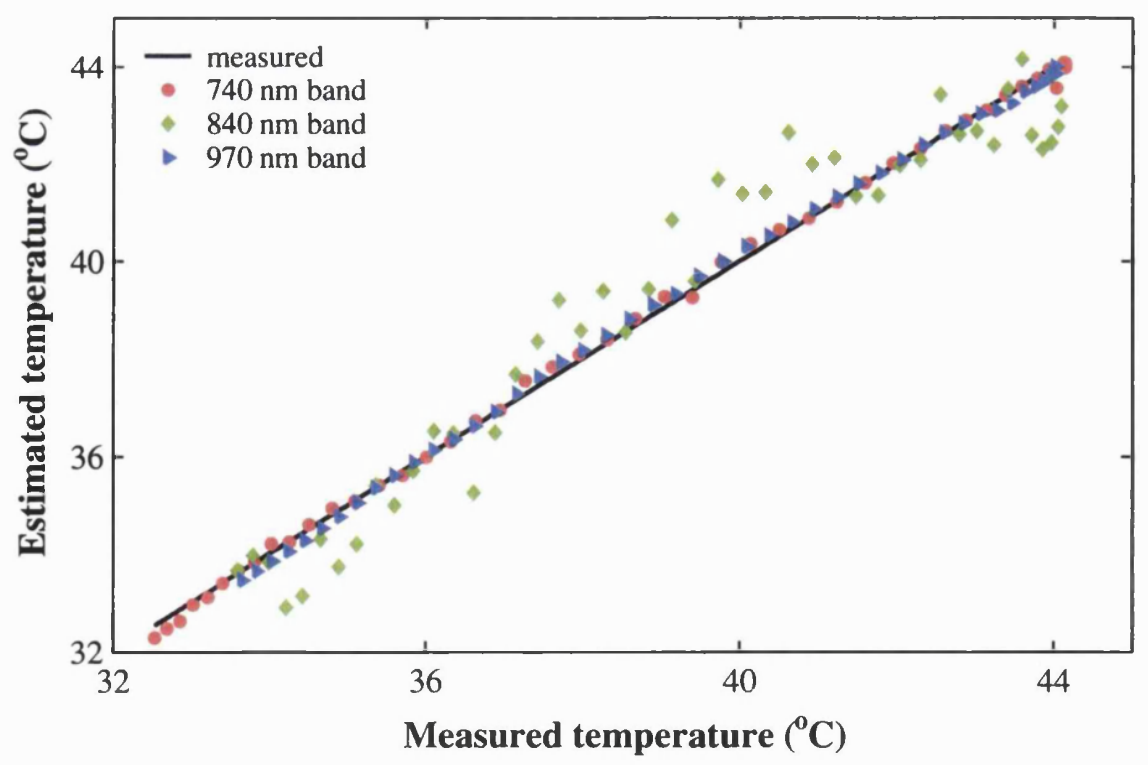

Figure 5.17: Estimated against measured temperature for the CLS temperature calibration of the three NIR water absorption bands, as measured using Method I. 


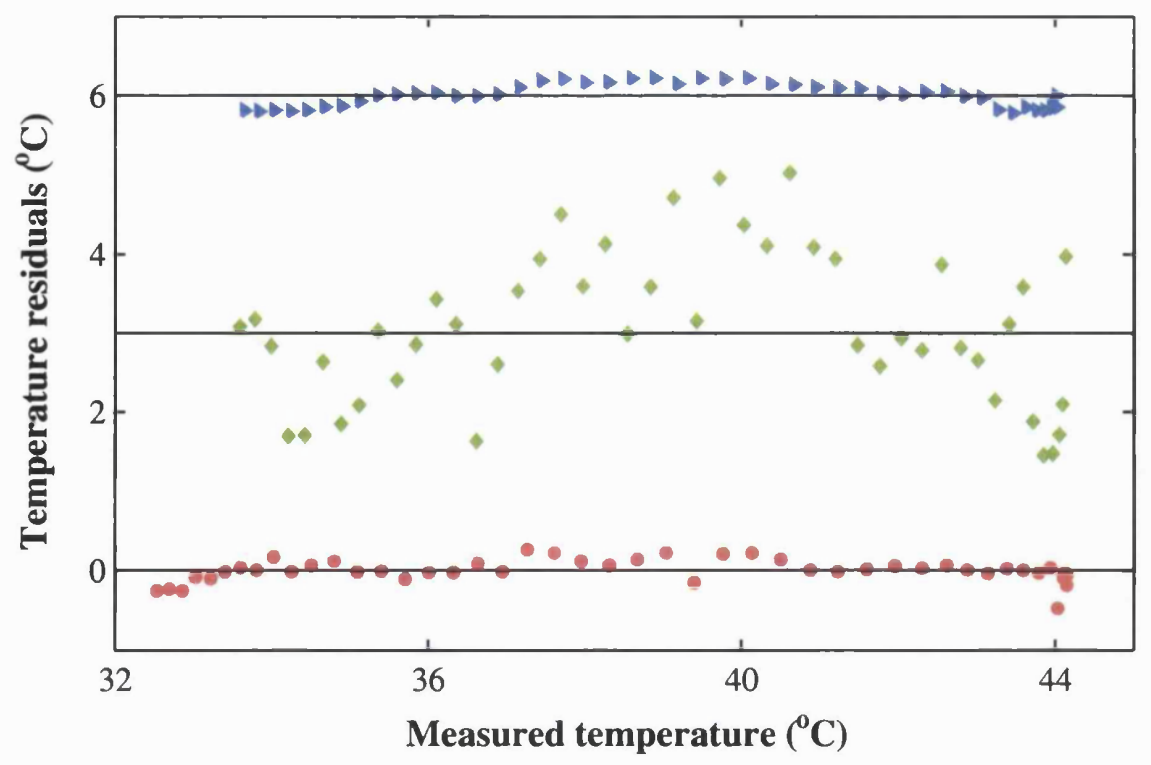

Figure 5.18: Temperature residuals for the CLS calibration of the NIR water absorption bands. Residuals are offset for clarity by 0,3 and $6{ }^{\circ} \mathrm{C}$ for the $740(\bullet), 840(\diamond)$ and 970 $(\triangleright) \mathrm{nm}$ bands respectively (solid black lines indicate offsets).

Figure 5.18 which shows the temperature residuals for each range (the residuals for the 840 and $970 \mathrm{~nm}$ bands are offset by 3 and $6{ }^{\circ} \mathrm{C}$ for clarity). Relative to the other two bands the $840 \mathrm{~nm}$ band exhibits a poor temperature prediction. This is probably due to the fact that the absolute errors on the $840 \mathrm{~nm}$ absorption band measurements are greater than those for the $740 \mathrm{~nm}$ band, although their temperature coefficients are similar, and whereas the errors for the $840 \mathrm{~nm}$ band are smaller than for the $970 \mathrm{~nm}$ band, the temperature coefficient of the latter is much greater (Figure 5.11). For all three ranges the residuals indicate a small degree of non-linearity that is not accounted for by this model.

Table 5.6 gives details of the standard errors and the $\mathrm{r}^{2}$ correlation coefficients for the temperature prediction. The standard error of calibration (SEC), given in Table 5.6, is defined as follows

$$
\mathrm{SEC}=\sqrt{\frac{\sum_{i}^{\mathrm{n}}\left(\mathrm{t}_{i}-\hat{\mathrm{t}}_{i}\right)^{2}}{\mathrm{n}-1}}
$$

\begin{tabular}{lllll}
\hline $\begin{array}{l}\text { Water } \\
\text { band }(\mathrm{nm})\end{array}$ & $\begin{array}{l}\mathrm{SEC} \\
\left({ }^{\circ} \mathrm{C}\right)\end{array}$ & $\mathrm{r}_{c}^{2}$ & $\begin{array}{l}\mathrm{SEP} \\
\left({ }^{\circ} \mathrm{C}\right)\end{array}$ & $\mathrm{r}_{p}^{2}$ \\
\hline 740 & 0.132 & 0.9989 & 0.135 & 0.9988 \\
840 & 0.951 & 0.9271 & 0.954 & 0.9261 \\
970 & 0.141 & 0.9983 & 0.142 & 0.9982 \\
\hline
\end{tabular}

Table 5.6: Statistical parameters for the CLS temperature calibration of the three NIR water absorption bands 
where the $t_{i} s$ are the measured temperatures and the $\hat{t}_{i} s$ are the model-predicted temperatures, as defined in Equation (5.30). The standard error of prediction (SEP) was determined using the cross-validation, or 'leave-one-out' method (Haaland and Thomas, 1988). The cross-validation method assesses the ability of the calibration to predict the temperature of unknown spectra by leaving these out of the calibration. A single spectrum is left out of the calibration process and a new calibration vector $\mathbf{p}$ is determined as before using the remaining spectra. The temperature for the 'left-out' spectrum is then predicted using the 'all-but-one' calibration vector. This process is repeated for each spectrum giv-

ing $\hat{\mathbf{t}}$ for the full data set. The SEP is then calculated in the same way as the SEC using Equation (5.31). Strictly this is not the same as prediction testing with a new independent data set, since the validation data is taken from the original calibration data set, but the method gives a reasonable idea of predictive ability of a calibration technique (Martens and Næs, 1991). The coefficients $\mathrm{r}_{c}^{2}$ and $\mathrm{r}_{p}^{2}$ in Table 5.6 give the correlation between the actual (measured) temperature and the temperatures estimated by full calibration and prediction (cross-validation) respectively.

\subsubsection{Inverse Least-Squares}

Although this method has not been used to calibrate the water absorption spectra measured during this project, it will briefly be mentioned since it is a popular method and will be referred to again in later sections. The inverse least-squares method (ILS), also known simply as MLR, applied to component concentration analysis assumes that concentration is a function of absorption, as opposed to the reverse with CLS. During the calibration, therefore, it is the errors in concentration that are minimised (Martens and Næs, 1991). The ILS calibration model for this 'inverse' Beer's law is given as

$$
\mathbf{C}=\mathbf{A} \cdot \mathbf{P}+\mathbf{E}_{\mathbf{C}}
$$

where $\mathbf{C}$ is the $(\mathrm{n} \times \mathrm{h})$ matrix of concentrations for $\mathrm{h}$ components and $\mathrm{n}$ spectra, $\mathbf{A}$ is the $(\mathrm{n} \times \mathrm{m})$ calibration data set as before (Section 5.3.2), $\mathbf{P}$ is the $(\mathrm{m} \times \mathrm{h})$ matrix relating the concentrations in $\mathbf{C}$ to the absorption values in $\mathbf{A}$ and $\mathbf{E}_{\mathbf{C}}$ is the $(\mathrm{n} \times \mathrm{h})$ concentration residuals matrix. An advantage of this method over CLS in the determination of component concentrations is that it can be applied to a single component, i.e. only knowledge of the concentration of the analyte of interest is required for the calibration, assuming that the component concentrations are independent of one another. For a single component, 
therefore, the least-squares solution of the calibration vector is

$$
\hat{\mathbf{p}}=\left(\mathbf{A}^{\prime} \cdot \mathbf{A}\right)^{-1} \cdot \mathbf{A}^{\prime} \cdot \mathbf{c}
$$

where $\mathbf{c}$ is the $(\mathrm{n} \times 1)$ vector of concentrations for the required component. A disadvantage of the method is that the matrix $\mathbf{A}$ must be inverted, therefore requiring that the number of wavelengths $m$ is less than the number of spectra $n$ in the data set. This often means that some information must be discarded, or selected, from the data set in order to provide the best calibration, which can be a complicated process. As noted by Haaland and Thomas (1988), including too many wavelengths in the regression, even within the $\mathrm{m}<\mathrm{n}$ limit, can degrade the results of the calibration due to a possible co-linearity (close-to-linear relationship) of the absorption values at different wavelengths.

For a temperature calibration the assumption would be that temperature $t$ is a function of absorption, and the ILS model would be given and followed through as

$$
\begin{aligned}
& \mathbf{t}=\mathbf{A} \cdot \mathbf{p}+\mathbf{e}_{\mathbf{t}} \\
& \hat{\mathbf{p}}=\left(\overline{\mathbf{A}}^{\prime} \cdot \overline{\mathbf{A}}\right)^{-1} \cdot \overline{\mathbf{A}}^{\prime} \cdot \mathbf{t} \\
& \hat{\mathbf{t}}=\overline{\mathbf{a}} \cdot \hat{\mathbf{p}}
\end{aligned}
$$

where $\overline{\mathbf{A}}$ is the reduced data set of absorption values at selected wavelengths, i.e. with $\mathrm{m}<\mathrm{n}, \mathrm{p}$ is the calibration vector relating temperature to absorption and $\mathbf{e}_{\mathbf{t}}$ are the temperature residuals. The least-squares solution to $\mathbf{p}$ is found by inverting the reduced data set matrix $\overline{\mathbf{A}}$ and can then be used to predict the temperature $\hat{t}$ of the sample with a given (reduced) spectrum $\overline{\mathbf{a}}$.

Therefore, although not as conceptually agreeable as the CLS method for temperature calibration, the ILS method has the advantage that wavelengths at which the correlation between absorption and temperature are poor can be disregarded, provided a suitable method of wavelength selection is used. Lin and Brown (1993) used ILS, with a 'stepforward' technique to select the most suitable wavelengths, to calibrate the water absorption spectrum from 1.11-1.87 $\mu \mathrm{m}$ against temperature. They found that the optimum number of wavelengths to include in the regression of temperature on to absorption was six. Beyond this number the SEC and the SEP for the calibration were seen to increase. Kelly et al (1995) calibrated the water bands at 970, 1200 and $1450 \mathrm{~nm}$ using ILS. For the $970 \mathrm{~nm}$ band they determined SECs of 0.12 and $0.11^{\circ} \mathrm{C}$ with two and three wavelengths included respectively. 


\subsubsection{Hybrid Method}

This calibration method is essentially a hybrid of both CLS and ILS techniques. The underlying assumption is that the water absorption spectrum at any temperature within a certain range can be described as a linear combination of two 'basis' spectra, measured at the upper and lower limit of the temperature range. The analysis for the general case of a variable number of basis spectra can be described as follows

$$
\mathbf{A}=\mathbf{C} \cdot \mathbf{B}+\mathbf{E}_{\mathbf{A}}
$$

where $\mathbf{A}$ and $\mathbf{E}_{\mathbf{A}}$ are as before, $\mathbf{B}$ is the $(\mathrm{h} \times \mathrm{m})$ matrix of $\mathrm{h}$ basis spectra and $\mathbf{C}$ is ( $\mathrm{n} \times$ h) matrix of coefficients relating the absorption spectra in $\mathbf{A}$ to the basis spectra in $\mathbf{B}$, i.e. the concentrations of the basis spectra in each absorption spectrum. The concentration matrix $\mathbf{C}$ is given by the least-squares regression of $\mathbf{A}$ on to $\mathbf{B}$

$$
\hat{\mathbf{C}}=\mathbf{A} \cdot \mathbf{B}^{\prime} \cdot\left(\mathbf{B} \cdot \mathbf{B}^{\prime}\right)^{-1}
$$

The next step is then to relate the sample temperature to the concentrations of the basis spectra B determined from Equation (5.36) as follows

$$
\mathbf{t}=\mathbf{C} \cdot \mathbf{v}+\mathbf{e}_{\mathrm{t}}
$$

where $\mathbf{t}$ and $\mathbf{e}_{\mathbf{t}}$ are as before and $\mathbf{v}$ is an $(h \times 1)$ vector of coefficients relating the $\mathbf{C}$ matrix to the temperature. The least-squares solution to $\mathbf{v}$ is given by

$$
\hat{\mathbf{v}}=\left(\hat{\mathbf{C}}^{\prime} \cdot \hat{\mathbf{C}}\right)^{-1} \cdot \hat{\mathbf{C}}^{\prime} \cdot \mathbf{t}
$$

where the solution for concentrations $\hat{\mathbf{C}}$ determined by Equation (5.36) is used. For an 'unknown' spectrum a the concentrations $\hat{\mathbf{c}}$ of the basis spectra in that sample are determined and temperature is predicted using the vector $\hat{\mathbf{v}}$ determined from the calibration step in Equation (5.38)

$$
\begin{aligned}
& \hat{\mathbf{c}}=\mathbf{a} \cdot \mathbf{B}^{\prime} \cdot\left(\mathbf{B} \cdot \mathbf{B}^{\prime}\right)^{-1} \\
& \hat{\mathbf{t}}=\hat{\mathbf{c}} \cdot \hat{\mathbf{v}}
\end{aligned}
$$

The step in Equation (5.36) is the CLS regression from Equation (5.29), except that $\mathbf{A}$ 


\begin{tabular}{lllll}
\hline $\begin{array}{l}\text { Water } \\
\text { band }(\mathrm{nm})\end{array}$ & $\begin{array}{l}\mathrm{SEC} \\
\left({ }^{\circ} \mathrm{C}\right)\end{array}$ & $\mathrm{r}_{c}^{2}$ & $\begin{array}{l}\mathrm{SEP} \\
\left({ }^{\circ} \mathrm{C}\right)\end{array}$ & $\mathrm{r}_{p}^{2}$ \\
\hline 740 & 0.102 & 0.9997 & 0.104 & 0.9993 \\
840 & 0.151 & 0.9990 & 0.153 & 0.9980 \\
970 & 0.108 & 0.9995 & 0.110 & 0.9990 \\
\hline
\end{tabular}

Table 5.7: Statistical parameters for the 'hybrid' temperature calibration of the three NIR water bands

is regressed on to the basis spectra in $\mathbf{B}$ instead of temperature. The concentrations are then related to temperature by the vector $\mathbf{v}$, the least-squares solution to which is given by Equation (5.38). This is the ILS step of Equation (5.33), except that the problems of inverting matrix $\mathbf{A}$ are avoided with matrix $\mathbf{C}$ since $\mathrm{h} \ll \mathrm{n}$, therefore no information need be discarded.

In the method proposed there are only two basis spectra, i.e. $\mathbf{B}$ is a $(2 \times \mathrm{m})$ matrix containing the water absorption spectra measured at upper and lower temperature limits. As with the CLS method, both the absorption and temperature data were mean-centred. The first and last spectra in each data set were used as the basis spectra for the calibration of each range. The results of the calibration are given in Table 5.7. The standard errors, calculated in the manner described in Section 5.3.2, are significantly reduced compared to those of the CLS calibration (Table 5.6), especially in the case of the $840 \mathrm{~nm}$ water band.

It is interesting to look at reasons why the hybrid method reduces the standard error

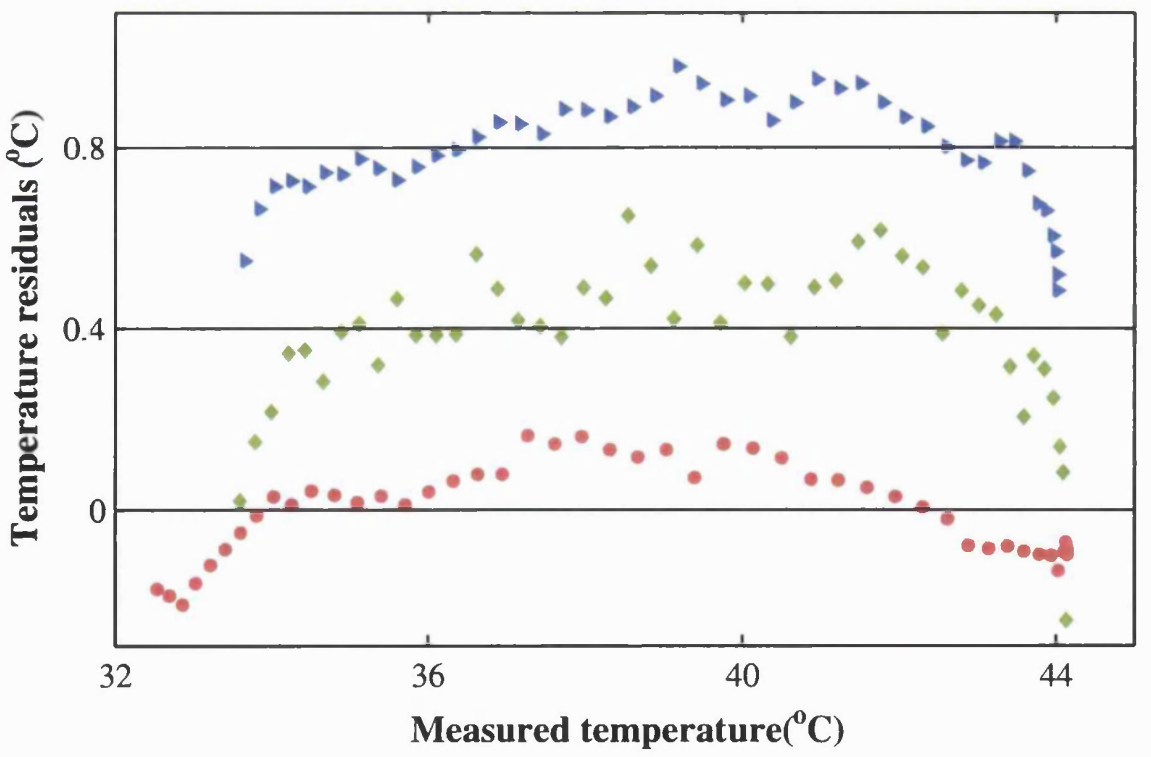

Figure 5.19: Temperature residuals for the 'hybrid' calibration of the NIR water absorption bands. Residuals are offset for clarity by $0,0.4$ and $0.8^{\circ} \mathrm{C}$ for the $740(\bullet), 840(\diamond)$ and $970(\triangleright) \mathrm{nm}$ bands respectively (solid black lines indicate offsets). 
relative to the CLS method. The approximation of Equation (5.35) essentially reduces the system $\mathbf{A}$ of $\mathrm{m}$ absorption intensities for each spectrum to a system in which there are only two intensities (in $\mathbf{C}$ ) operating on a set of two fixed basis spectra (B). This process of data compression reduces the total amount of noise in the new system (Geladi and Kowalski, 1986; Haaland and Thomas, 1988). In the prediction step, the less noisy $\hat{\mathbf{c}}$ and $\hat{\mathbf{v}}$ in Equation (5.38) replace a and $\mathbf{p}$ (or rather $\hat{\mathbf{p}}^{\prime} \cdot\left(\hat{\mathbf{p}} \cdot \hat{\mathbf{p}}^{\prime}\right)^{-1}$ ) in Equation (5.30). The temperature values predicted by the hybrid calibration method will therefore have smaller errors than the values determined by the CLS method. Figure 5.19 shows the temperature residuals against the measured temperature for the three bands (again offset for clarity). In comparison with the temperature residuals of the CLS method (Figure 5.18) the error on the predicted values appears to be reduced. The non-linearity in the temperature is still present, albeit to a smaller extent than for the CLS method, indicating that the model has not described the full nature of the dependence of absorption on temperature. (Note the use of a different scale in the offsets between Figures 5.18 and 5.19).

\subsubsection{Principal Component Regression}

\subsubsection{Theory}

The next calibration to be applied to the water absorption spectra was the technique of principal component regression (PCR). PCR is known as a bilinear method because in the initial step the measured data set $(\mathbf{A})$ is decomposed into two sets of linear (in $\mathbf{A}$ ) parameters (Martens and Næs, 1991). The aim is to reduce the dimensionality of the original data set by discarding those parameters that contribute only noise and keep those that describe the majority of the variance in the data. The new reduced data set is then used in the regressional calibration of the dependent variable.

In PCR the initial step is known as principal component analysis (PCA). The data set $\mathbf{A}$ comprised of many independent variables (e.g. absorptions at many wavelengths) is compressed to a smaller number of variables known as principal components (PCs). In matrix notation PCA can be described as follows

$$
\mathbf{A}=\mathbf{S} \cdot \mathbf{L}+\mathbf{E}_{\mathbf{A}}
$$

where the $(\mathrm{n} \times \mathrm{m})$ data matrix $\mathbf{A}$ is represented by the product of the $(\mathrm{n} \times \mathrm{h})$ matrix $\mathbf{S}$ and the $(\mathrm{h} \times \mathrm{m})$ matrix $\mathbf{L}$, where $\mathrm{h}$ is the number of PCs used in the model, and $\mathbf{E}_{\mathrm{A}}$ is the $(\mathrm{n} \times \mathrm{m})$ residual matrix. The $(\mathrm{n} \times 1)$ column vectors of the $\mathbf{S}$ matrix are known as 
the scores and the $(1 \times \mathrm{m})$ row vectors of matrix $\mathbf{L}$ are called the loadings, for each 1 to h PCs. It is the scores matrix $\mathbf{S}$ that is later used in the regression with the dependent variable, as with the hybrid method described by Equations (5.35)-(5.39). However, with PCR the basis spectra, i.e. the $(1 \times \mathrm{m})$ loading vectors, are specifically determined such that they account for the maximum possible variance in $\mathbf{A}$, as opposed to being chosen arbitrarily as was the case with the hybrid method.

A graphical representation of PCA is given in Figure 5.20 for an arbitrary data set with two independent variables, i.e. $\mathbf{A}$ is an $(\mathrm{n} \times 2)$ matrix. The two variables, represented by the column vectors $\mathbf{a}_{1}$ and $\mathbf{a}_{2}$, are plotted against one another. The PC is the line of best fit through the data points, i.e. that which minimises the sum of squares of the $\mathbf{a}_{1}$ and $\mathbf{a}_{2}$ residuals and hence describes the greatest amount of variance between the two variables (Geladi and Kowalski, 1986). The $\mathrm{n}$ scores (also known as intensities) are the projections of the data points on to the $\mathrm{PC}$, and the elements of the $(1 \times 2)$ loading vector are the direction cosines of the $\mathrm{PC}$, i.e. the projections of a unit vector along the $\mathrm{PC}$ on to the axes. For the example in Figure 5.20 the inclusion of only one PC will be sufficient to describe the vast majority of the variance in the data, since the regression line (the PC) is clearly a reasonable fit. In the case where there are many $(\mathrm{m})$ independent variables, more PCs may be required to model the data matrix A. Subsequent PCs should be chosen carefully in order to ensure a high degree of uniqueness among them (Helland, 1988). A common
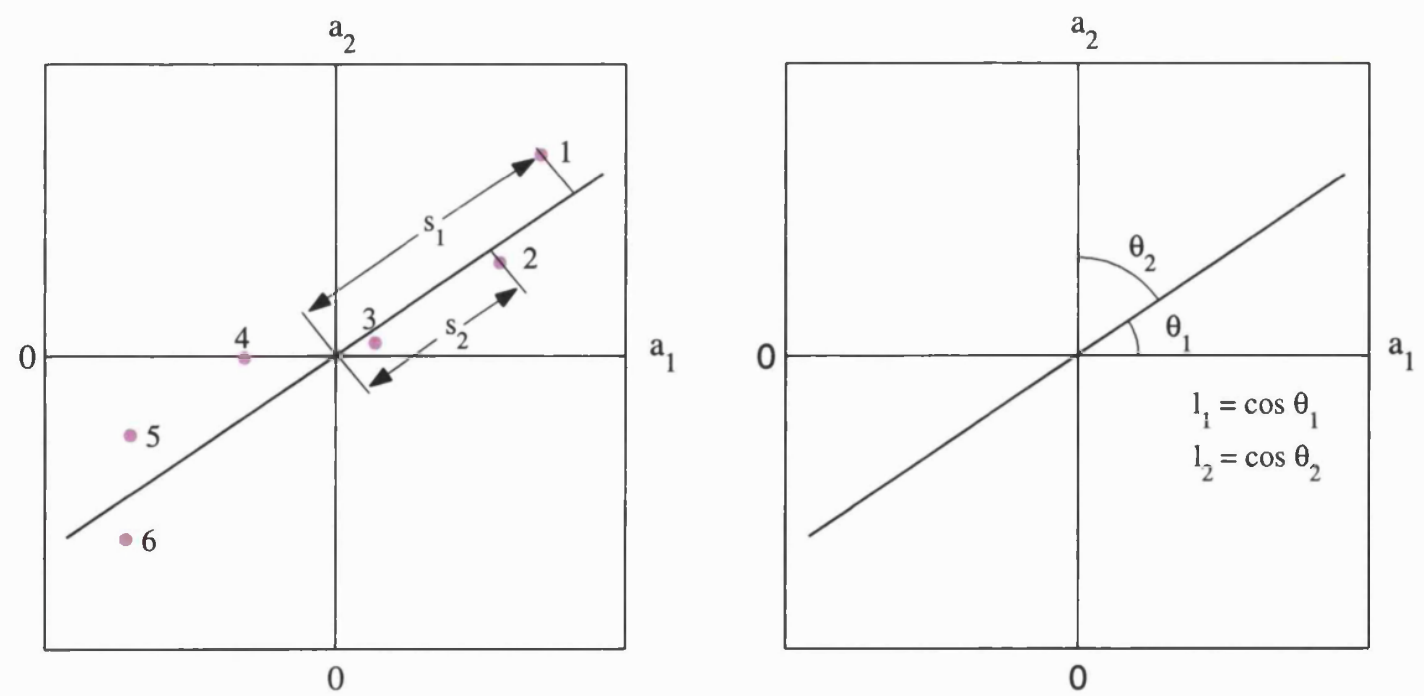

Figure 5.20: A graphical representation of PCA in the case of two independent variables. Left: Variables $a_{1}$ and $a_{2}$ plotted against one another. The black line shows the leastsquares regression through the points, representing the PC. The scores $\mathrm{s}_{1}$ and $\mathrm{s}_{2}$ are given by the projections of data points 1 and 2 onto the PC (six in total). Right: The loadings $l_{1}$ and $l_{2}$ are given by the direction cosines of the PC. See text for details. 
choice is to impose the condition that subsequent scores are orthogonal to one another, and likewise for the loadings (Helland, 1988; Haaland and Thomas, 1988). Equation (5.40) can be rewritten as follows

$$
A=s_{1} \cdot l_{1}+s_{2} \cdot l_{2}+\ldots+s_{h} \cdot l_{h}+E_{A}
$$

If, for $i=1$ to $\mathrm{h}$, the $(\mathrm{n} \times 1)$ score vectors $\mathrm{s}_{i}$ are orthogonal to one another, and the $(1 \times \mathrm{m})$ loading vectors $l_{i}$ are also orthogonal, then the score vectors are eigenvectors of $\left(\mathbf{A} \cdot \mathbf{A}^{\prime}\right)$ and the loading vectors are eigenvectors of $\left(\mathbf{A}^{\prime} \cdot \mathbf{A}\right)$ (Helland, 1988; Martens and Næs, 1991). Since this statement is not demonstrated mathematically in the literature a brief proof is given in Appendix B in order to establish a basis for the subsequent method of decomposing the A matrix.

There are a variety of algorithms for calculating the scores and loadings in PCA (Otto, 1998). In this case, a built-in MATLAB function was used to extract the eigenvectors of the (mean-centred) matrix $\left(\mathbf{A}^{\prime} \cdot \mathbf{A}\right)$, which gave the loadings matrix $\mathbf{L}$. The loading vectors determined in this way are orthonormal; that is, they are orthogonal and are also normalised to unit length, such that $\mathbf{l}_{i} \cdot \mathbf{l}_{j}=1$ for $i=j$ and $\mathbf{l}_{i} \cdot \mathbf{l}_{j}=0$ for $i \neq j$. The scores matrix is then determined as follows

$$
\begin{aligned}
& \mathbf{S}=\mathbf{A} \cdot \mathbf{L}^{\prime} \cdot\left(\mathbf{L} \cdot \mathbf{L}^{\prime}\right)^{-1} \\
& \mathbf{S}=\mathbf{A} \cdot \mathbf{L}^{\prime}
\end{aligned}
$$

where the orthonormality of matrix $\mathbf{L}$ ensures that the product $\left(\mathbf{L} \cdot \mathbf{L}^{\prime}\right)$ is equal to the diagonal identity matrix I and hence disappears.

The next stage of the PCR calibration is to establish a linear relationship between the PCA scores and the dependent variable, i.e. temperature. The model is defined as follows

$$
\mathbf{t}=\mathbf{S} \cdot \mathbf{v}+\mathbf{e}_{\mathrm{t}}
$$

where $\mathbf{t}$ contains the measured temperatures (also mean-centred), $\mathbf{v}$ is the vector relating the scores $\mathbf{S}$ to the temperature and as before $\mathbf{e}_{\mathbf{t}}$ are the temperature residuals not fit by the model. A solution for the calibration vector is found in the least-squares sense by regression of the measured temperature $\mathbf{t}$ on to the scores in $\mathbf{S}$

$$
\hat{\mathbf{v}}=\left(\mathbf{S}^{\prime} \cdot \mathbf{S}\right)^{-1} \cdot \mathbf{S} \cdot \mathbf{t}
$$


One can see that for this method the problems of co-linearity associated with inversion of the data matrix $\mathbf{A}$ (discussed in Section 5.3.3) are eliminated, since the columns of $\mathbf{S}$ are orthogonal and therefore completely uncorrelated.

In prediction, the spectrum a (from which the mean calibration spectrum is subtracted) of an 'unknown' sample is regressed on to the loadings matrix $\mathbf{L}$, determined from the full PCR calibration, to give the $\mathrm{h}$ scores for that spectrum

$$
\mathbf{s}=\mathbf{a} \cdot \mathbf{L}^{\prime}
$$

as for the calibration step in Equation (5.42). The temperature is then given by

$$
\hat{t}=\mathbf{s} \cdot \hat{\mathbf{v}}+\bar{t}
$$

where $\hat{\mathbf{v}}$ is the calibration vector given in Equation (5.44), determined during the full calibration, and $\overline{\mathrm{t}}$ is the mean calibration temperature.

\subsubsection{Results}

As with the previous calibration methods the analysis was performed on each of the three spectral ranges of the water absorption spectrum, measured using Method I. For each range the full $\mathrm{h}=\mathrm{m}$ eigenvectors of $\left(\mathbf{A}^{\prime} \cdot \mathbf{A}\right)$ were found and returned in order of descending eigenvalue size, i.e. the first loading vector describes the maximum variance

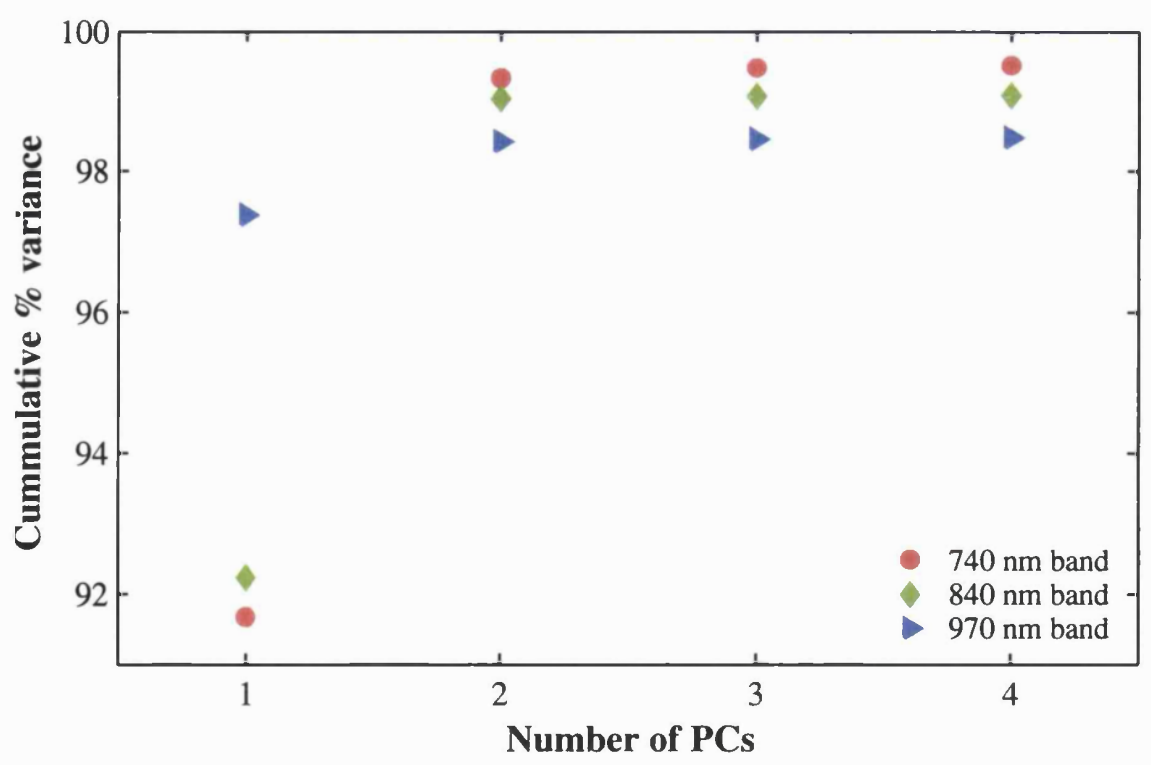

Figure 5.21: Cumulative percentage of the total variance in the water absorption spectra for the first four PCs. See text for details. 


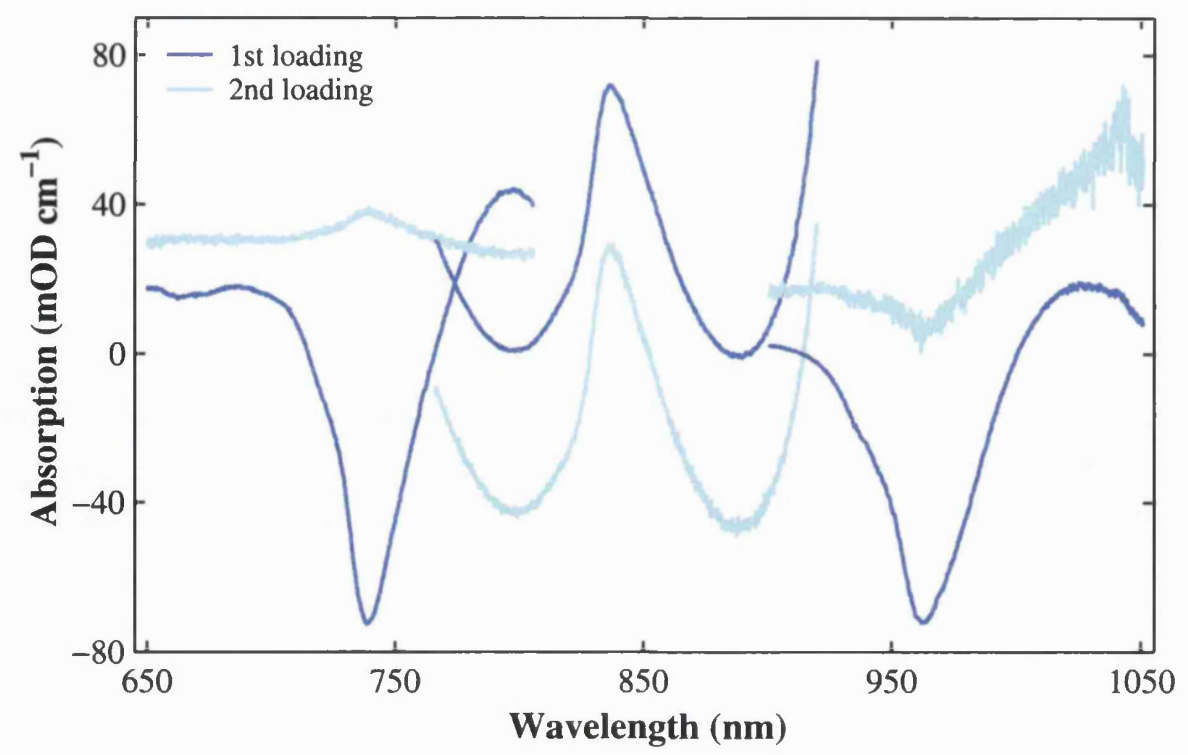

Figure 5.22: The first and second loadings of the three NIR water absorption bands measured as a function of temperature, as determined by PCA

in $\mathbf{A}$, the second describes the maximum of the remaining variance and so on. The percentage of the total variance in the mean-centred data set was examined by determining the cumulative percentage of the sum of all the eigenvalues for each additional eigenvalue (i.e. PC). Figure 5.21 shows the cumulative percentage of the total variance for the first four PCs for each range. The first PC accounted for well over $90 \%$ of the total variance in each band. Including a second PC accounted for more than $99 \%$ of the variance in the 740 and $840 \mathrm{~nm}$ bands and over $98 \%$ of the variance in the $970 \mathrm{~nm}$ band. The first and second loading vectors for each range are shown in Figure 5.22.

\begin{tabular}{llllll}
\hline $\begin{array}{l}\text { Water } \\
\text { band }(\mathrm{nm})\end{array}$ & PCs & $\begin{array}{l}\mathrm{SEC} \\
\left({ }^{\circ} \mathrm{C}\right)\end{array}$ & $\mathrm{r}_{c}^{2}$ & $\begin{array}{l}\mathrm{SEP} \\
\left({ }^{\circ} \mathrm{C}\right)\end{array}$ & $\mathrm{r}_{p}^{2}$ \\
\hline 740 & 1 & 0.1360 & 0.9988 & 0.1387 & 0.9988 \\
& 2 & 0.1053 & 0.9993 & 0.1068 & 0.9993 \\
& 3 & 0.1008 & 0.9993 & 0.1023 & 0.9993 \\
& 4 & 0.1003 & 0.9994 & 0.1022 & 0.9993 \\
840 & 1 & 0.9875 & 0.9152 & 0.9943 & 0.9140 \\
& 2 & 0.1411 & 0.9983 & 0.1428 & 0.9982 \\
& 3 & 0.1021 & 0.9991 & 0.1050 & 0.9990 \\
& 4 & 0.0501 & 0.9998 & 0.0567 & 0.9997 \\
970 & 1 & 0.1416 & 0.9983 & 0.1428 & 0.9982 \\
& 2 & 0.0996 & 0.9991 & 0.1008 & 0.9991 \\
& 3 & 0.0444 & 0.9998 & 0.0475 & 0.9998 \\
& 4 & 0.0444 & 0.9998 & 0.0477 & 0.9998 \\
\hline
\end{tabular}

Table 5.8: Statistical parameters for the PCR calibration of the NIR water bands 
It is important to determine the number of useful components to include in the prediction of the dependent variable. Including a large number of PCs will not improve the temperature prediction beyond a certain point since the later components are small and will contribute only noisy information. One method of determining the optimal number of PCs to use is to examine the goodness-of-fit of the model as a function of the number of PCs via its effect on the standard errors of calibration and prediction. Table 5.8 gives the SEC, SEP and correlation coefficients for the fits for the inclusion of up to four PCs, for each band. For the $740 \mathrm{~nm}$ water band the fit is not greatly improved beyond the inclusion of two PCs, for which the SEP is approximately $0.11^{\circ} \mathrm{C}$. For the $840 \mathrm{~nm}$ band three PCs are required to predict temperature with an SEP of approximately $0.11^{\circ} \mathrm{C}$. At the $970 \mathrm{~nm}$ band the SEP is $0.10^{\circ} \mathrm{C}$ for the inclusion of two PCs. As a comparison the results of Lin and Brown (1993) were examined. They studied the NIR water spectrum from $1.11-1.87 \mu \mathrm{m}$ in the temperature range $5-85^{\circ} \mathrm{C}$. Fitting to the whole spectral range they determined that 5-7 PCs were required for an SEP of between 0.16 and $0.32{ }^{\circ} \mathrm{C}$. The authors note that the noise on the data was relatively large due to the small pathlengths employed (0.4-1.2 mm).

The temperature residuals, shown in Figure 5.23, are also important in deciding upon the goodness-of-fit. For the 840 and $970 \mathrm{~nm}$ bands one can see that the progression from two to three PCs not only reduces the random errors but also improves the fit in

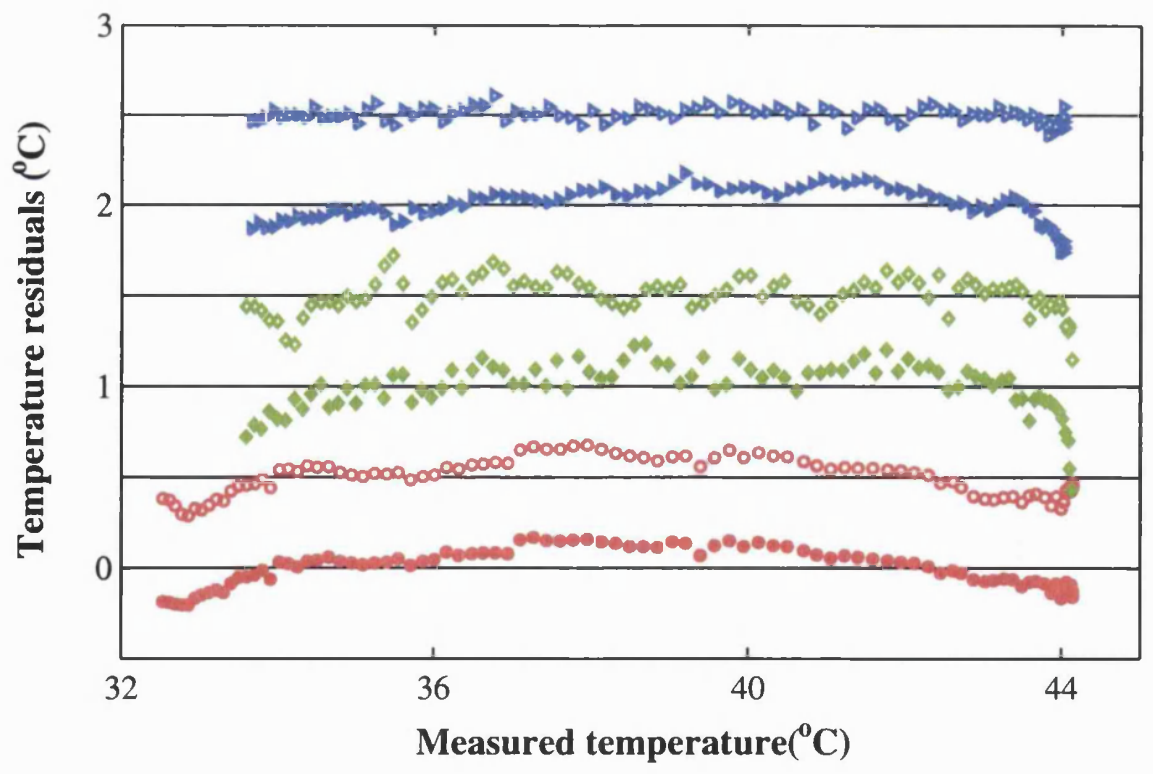

Figure 5.23: Temperature residuals for PCR calibration with 2 and 3 PCs of the NIR water absorption bands at 740,840 and $970 \mathrm{~nm}$. Closed and open symbols are the residuals determined with the inclusion of 2 and 3 PCs respectively. Residuals are offset for clarity, solid black lines indicate offset. 
terms of its agreement with the model, i.e. the non-linearity is reduced. For the $740 \mathrm{~nm}$ band, however, little improvement in the fit is seen when three PCs are included, (as is also indicated by the standard errors for this range in Table 5.8), and the inclusion of additional PCs had no further effect on the residuals.

The temperature analysis above assumes that the concentration of the 'unknown' data set is the same as that of the calibration set. This is of course true in this case, since the unknown data set and the calibration set are the same. However, using this calibration to predict temperature from a truly unknown sample in which the exact water concentration may also be unknown, e.g. biological tissue, requires careful consideration. In order to correctly predict temperature from the unknown spectrum using Equations (5.45) and (5.46), the mean calibration spectrum must be subtracted at the concentration at which it should contribute to the unknown sample, i.e. at the water concentration of the unknown sample. Since the water concentration may not always be $100 \%$, as was the case for the calibration data set, the mean spectrum must be included as an additional component in the regression of the unknown spectrum against the loading vectors. The corresponding scores must then be normalised to the concentration of the mean spectrum in order to recover the validity of their relationship to temperature described by Equation (5.46). Since the mean spectrum is not orthogonal to the loading vectors, including it in the regression will affect the accuracy with which the scores are recovered. This phenomenon is examined in closer detail in Appendix C, where it is shown that its effect on the temperature prediction is greater when less PCs are included in the model, but does not depend on water concentration.

The PCR model has an advantage over the preceding multivariate calibration techniques in that it may describe more closely the physical possibility that several temperature-dependent components are causing the variation in the water spectrum, the underlying spectra of which are directly related to the loading vectors. For this reason, and because it gave the smallest temperature residuals and standard errors, PCR was chosen over the methods described in Sections 5.3.2-5.3.4 to be used in the temperature prediction algorithms (see Sections 7.1 and 7.2). It should be noted, though, that if the variation in absorption is due to a continual shift in hydrogen-bonding strengths with temperature then the PCR model may be altogether unsuitable, especially if these shifts are large (Martens, 2000). In truth, all the calibration methods presented here, including PCR, attempt to model an essentially non-linear spectral change as a combination of linear effects. 
A major disadvantage of PCR as a calibration method is the possibility that a component that describes a large amount of variance in the data, i.e. has a large eigenvalue, may not be well correlated to the dependent variable (Haaland and Thomas, 1988; Martens and Næs, 1991). In other words, in addition to a change in temperature there may be some other physical or chemical effect occurring that also causes a significant change in the water absorption spectrum. For this reason a further method of calibrating the water spectrum for temperature was investigated to be used in a later temperature prediction algorithm (see Section 7.3), as will be described in the following section.

\subsubsection{Partial Least Squares Regression}

\subsubsection{Theory}

The final calibration technique to be discussed is that of partial least squares regression (PLSR). Like PCR (Section 5.3.5), PLSR is a bilinear technique in that it decomposes the calibration matrix into two smaller matrices, as was represented in Equation (5.40). However, it differs from PCR in its approach to the reduction of the original data set, and as a result has several potential advantages over PCR.

The PCR algorithm aims firstly to reduce the dimensionality of the data matrix and describe as much of the true variance as possible with the reduced data set, i.e. the residuals in matrix $\mathbf{E}_{\mathrm{A}}$ are minimised. The scores determined from this process (PCA) are then used in the regression step to calibrate the dependent variable. However, the scores that minimise the spectral residuals in Equation (5.40) may not optimally minimise the temperature residuals $\mathbf{e}_{t}$ in Equation (5.43) (Helland, 1988). The objective of the PLSR method is to improve the correlation between the spectral scores and the dependent variable, albeit at the expense of the spectral fit described by the model in Equation (5.40). This makes it a much more robust calibration than the PCR method (Geladi and Kowalski, 1986; Otto, 1998), i.e. the parameters of the calibration model remain more stable when new data sets are taken from a total population. Moreover, PLSR performs the calibration in one step, as opposed to two as with PCR, therefore there is less chance of discarding useful information in unwanted components or factors (Geladi and Kowalski, 1986).

The PLSR algorithm used here was developed by Wold and Martens in the 1980's and and has been discussed in detail by Martens and Næs (1991). The method can be described using the same bilinear, principal component-type model as for PCR, given in Equation (5.41). However, since the scores and loadings are calculated differently, the 
principal components for PLSR are often known as latent variables or factors (Helland, 1988). The first score vector for PLSR can be written as a linear combination of the independent variables in $\mathbf{A}$

$$
\mathbf{s}_{1}=\mathbf{A} \cdot \mathbf{w}_{1}^{\prime}
$$

where the $(1 \times \mathrm{m})$ vector $\mathbf{w}_{1}$ is called the weight vector, i.e. it gives the weights with which the independent variables are combined to give the scores. In PCR this weight vector is the (normalised) loading vector $\mathbf{l}_{1}$ and is determined solely from the information in the data set A. In PLSR, however, the aim is to produce a high correlation between the scores and the dependent variable. Thus the weight vector is made proportional to the covariance between the independent variables in $\mathbf{A}$ and the dependent variable temperature

$$
\mathbf{w}_{1}=\mathbf{t}^{\prime} \cdot \mathbf{A}
$$

As with PCR the weight vector is normalised such that $\mathbf{w}_{1} \cdot \mathbf{w}_{1}^{\prime}=1$. Once the normalised weight vector has been determined the score vector $\mathbf{s}_{1}$ is then calculated using Equation (5.47). The score vector is then related to the measured temperature by the scalar $\mathrm{v}_{1}$, which is determined in the least-squares sense by regression of temperature on to the first score vector

$$
\hat{\mathbf{v}}_{1}=\left(\mathbf{s}_{1}^{\prime} \cdot \mathbf{s}_{1}\right)^{-1} \cdot \mathbf{s}_{1}^{\prime} \cdot \mathbf{t}
$$

In the so-called 'orthogonalised' PLSR algorithm (Martens and Næs, 1991), which is used here, an additional step is performed based on the bilinear scores and loadings model given in Equation (5.41)

$$
\mathbf{l}_{1}=\left(\mathbf{s}_{1}^{\prime} \cdot \mathbf{s}_{1}\right)^{-1} \cdot \mathbf{s}_{1}^{\prime} \cdot \mathbf{A}
$$

where $l_{1}$ is again the first loading vector. The purpose of this step will be explained shortly. The parameters for the next factor are then calculated using the residuals of the $\mathbf{A}$ and $\mathbf{t}$ matrices, which are given in the general case at the end of the $i$ th iteration by

$$
\begin{array}{rlr}
\mathbf{A}_{i}=\mathbf{A}_{i-1}-\mathbf{s}_{i} \cdot \mathbf{l}_{i} & {\left[\mathbf{A}_{0}=\mathbf{A}\right]} \\
\mathbf{t}_{i}=\mathbf{t}_{i-1}-\mathbf{s}_{i} \cdot \mathbf{v}_{i} & {\left[\mathbf{t}_{0}=\mathbf{t}\right]}
\end{array}
$$


The parameters for the $i$ th factor are then generated as follows

$$
\begin{aligned}
\mathbf{w}_{i} & =\mathbf{t}_{i}^{\prime} \cdot \mathbf{A}_{i-1} \quad\left[\mathbf{w}_{i}=\mathbf{w}_{i} \cdot\left(\mathbf{w}_{i} \cdot \mathbf{w}_{i}^{\prime}\right)^{-1}\right] \\
\mathbf{s}_{i} & =\mathbf{A}_{i-1} \cdot \mathbf{w}_{i}^{\prime} \\
\mathbf{v}_{i} & =\left(\mathbf{s}_{i}^{\prime} \cdot \mathbf{s}_{i}\right)^{-1} \cdot \mathbf{s}_{i}^{\prime} \cdot \mathbf{t}_{i-1} \\
\mathbf{l}_{i} & =\left(\mathbf{s}_{i}^{\prime} \cdot \mathbf{s}_{i}\right)^{-1} \cdot \mathbf{s}_{i}^{\prime} \cdot \mathbf{A}_{i-1}
\end{aligned}
$$

In the general case the steps in Equations (5.51) and (5.56) ensure that the score vectors are orthogonal to one another, which is important for removing the co-linearity problem discussed previously (see Section 5.3.2). A brief proof of the orthogonality of the scores using this method is given for the first two score vectors in Appendix D.

The iterative algorithm described by Equations (5.51)-(5.56) can also include prediction of the dependent variable for either the calibration data set (i.e. for determination of the SEC) or for an 'unknown' spectral measurement or set of measurements (Martens and Næs, 1991). For example, in the case of a single spectrum a (minus the mean calibration spectrum), the $i$ scores are estimated iteratively from the spectral residuals as follows

$$
\begin{aligned}
\mathrm{s}_{i} & =\mathbf{a}_{i-1} \cdot \mathbf{w}_{i}{ }^{\prime} \\
\text { where } \quad \mathrm{a}_{i} & =\mathbf{a}_{i-1}-\mathrm{s}_{i} \cdot \mathbf{l}_{i} \quad\left[\mathrm{a}_{0}=\mathbf{a}\right]
\end{aligned}
$$

In the prediction step, the weight and loading vectors determined from the calibration are used and the score vector is estimated from the unknown sample measurement $\mathbf{a}$. The predicted temperature for the inclusion of $\mathrm{h}$ factors is thus given by

$$
\hat{\mathrm{t}}_{\mathrm{h}}=\sum_{i=1}^{h} \mathrm{~s}_{i} \cdot \hat{\mathrm{v}}_{i}+\overline{\mathrm{t}}
$$

where the $\hat{v}_{i}$ 's are determined from the full calibration and as before $\bar{t}$ is the mean calibration temperature. Normally, though, prediction of the dependent variable for a new set of spectral measurements would be performed separately from (and subsequent to) the calibration process. Rather than run through the iterative prediction algorithm described above, a concise method for predicting temperature for the inclusion of $\mathrm{h}$ factors, the proof of which has been shown elsewhere (Helland, 1988), is given by

$$
\hat{\mathrm{t}}=\mathbf{a} \cdot \mathbf{W}^{\prime} \cdot\left(\mathbf{L} \cdot \mathbf{W}^{\prime}\right)^{-1} \cdot \hat{\mathbf{v}}+\overline{\mathrm{t}}
$$


where $\mathbf{W}$ is the $(\mathrm{h} \times \mathrm{m})$ weights matrix, $\mathbf{L}$ is the $(\mathrm{h} \times \mathrm{m})$ loadings matrix and $\hat{\mathbf{v}}$ is the $(h \times 1)$ vector relating the scores to the measured temperature, all of which are determined during the full PLSR calibration in Equations (5.51)-(5.56). As for the PCR calibration described in Section 5.3.5, when using the PLSR weight and loading vectors to predict the temperature of an unknown sample, the mean calibration spectrum must be included in the regression on to the unknown spectrum, and the corresponding scores must be normalised to the thus determined water concentration (see also Appendix C).

\subsubsection{Results}

The PLSR algorithm was applied to the water absorption spectra as measured by Method II (Section 5.1.2.3) in the range 650-925 $\mathrm{nm}$. Since the temperature prediction algorithms to be described later involve only wavelengths up to around $900 \mathrm{~nm}$, the $900-1050 \mathrm{~nm}$ range measured using Method II was not calibrated using PLSR. For comparison the PCR calibration method (Section 5.3.5) was also applied to the Method II data set, and the results of both are discussed here.

For PCR and PLSR a vast amount of both quantitative and qualitative information about the goodness-of-fit of the model can be gained by looking at the various calibration (and prediction) parameters. For the application of PCR to data set I (i.e. measured using Method I) in Section 5.3.5.2, these included the cumulative percentage variance in the spectral data, the standard errors of calibration and prediction (SEC and SEP) and the temperature residuals. For further qualitative information the loading vectors were also examined. A large loading for a given wavelength (and PC) indicates a significant variation in the attenuation at that wavelength. As was shown in Figure 5.22, as more PCs are included the later loading vectors are seen to contain less information about variation in the data and more noise, hence the relative importance of including these PCs in the model can be ascertained visually.

With the PLSR calibration one can obtain similarly useful information about the goodness-of-fit from the standard errors and the residuals. However, the loading vectors obtained from PLSR do not represent the maximum variance in the spectral data as with PCR, but have a rather different interpretation. As noted by Haaland and Thomas (1988), the steps in Equations (5.53) and (5.54) for the determination of the first weight and score vectors are identical to the CLS method described in Section 5.3.2. This means that the first weight vector attempts to represent the underlying (mean-centred) spectrum that varies linearly with temperature and the first score vector therefore represents a first- 


\begin{tabular}{l|llll|ll|ll}
\hline \multirow{2}{*}{$\begin{array}{l}\text { PCs } \\
\text { factors }\end{array}$} & \multicolumn{3}{|c|}{ Cumulative \% variance in data } & \multirow{2}{*}{ SEC $\left({ }^{\circ} \mathrm{C}\right)$} & \multicolumn{2}{|c}{$\mathrm{r}_{c}^{2}$} \\
\cline { 2 - 5 } & \multicolumn{2}{|c|}{ spectral } & \multicolumn{2}{c|}{ temperature } & & & & \\
\cline { 2 - 7 } 1 & PCR & PLSR & PCR & PLSR & PCR & PLSR & PCR & PLSR \\
\hline 2 & 98.7250 & 98.0179 & 98.6143 & 98.6463 & 0.3983 & 0.3936 & 0.9861 & 0.9865 \\
3 & 99.8824 & 99.1672 & 99.9890 & 99.9891 & 0.0356 & 0.0354 & 0.9999 & 0.9999 \\
4 & 99.9455 & 99.2218 & 99.9893 & 99.9915 & 0.0349 & 0.0311 & 0.9999 & 0.9999 \\
5 & 99.9540 & 99.2364 & 99.9904 & 99.9969 & 0.0331 & 0.0188 & 0.9999 & 1.0000 \\
6 & 99.9623 & 99.2411 & 99.9907 & 99.9989 & 0.0327 & 0.0113 & 0.9999 & 1.0000 \\
7 & 99.9701 & 99.2457 & 99.9907 & 99.9996 & 0.0327 & 0.0072 & 0.9999 & 1.0000 \\
8 & 99.9778 & 99.2501 & 99.9907 & 99.9998 & 0.0327 & 0.0049 & 0.9999 & 1.0000 \\
9 & 99.9853 & 99.2541 & 99.9908 & 99.9999 & 0.0324 & 0.0032 & 0.9999 & 1.0000 \\
10 & 99.9928 & 99.2581 & 99.9909 & 100.0000 & 0.0323 & 0.0022 & 0.9999 & 1.0000 \\
\hline
\end{tabular}

Table 5.9: Comparison of temperature calibration parameters obtained from the application of PCR and PLSR to temperature-dependent NIR water absorption spectra. Both methods were applied to data set II, including up to $10 \mathrm{PCs}$ or factors in the model.

order approximation of the (mean-centred) temperatures. Inclusion of further factors in the model allows for the possibility that more than one 'component' varies spectrally (albeit linearly) with temperature. The loading vectors for PLSR, as determined by Equation (5.56), therefore attempt to account for as much variation in the spectral data as possible whilst simultaneously maximising the correlation with the scores, which are a representation of the dependent variable. Whereas with PCR any significant variation in attenuation produces large loadings, with PLSR they are related specifically to temperature-sensitive regions of the spectrum. Although, as will be shown, they are in practise fairly similar, the PLSR weight vectors give a better depiction of the spectral temperature-dependence of the factors than the corresponding loading vectors, since they are more directly related to temperature as opposed to a representation of temperature (the scores).

Table 5.9 gives the following calibration parameters as a function of the number of PCs or factors (up to ten) included in the model for both PCR and PLSR: the cumulative percentage variance of the total variance in the spectral data, the cumulative percentage variance of the total variance in the temperature data, the SEC and the correlation coefficient for calibration. As can be seen in the second and third columns, for a given number of PCs or factors the PCR method always describes a greater percentage of the total variance in the spectral data. As the model dimension increases the percentage of the total 

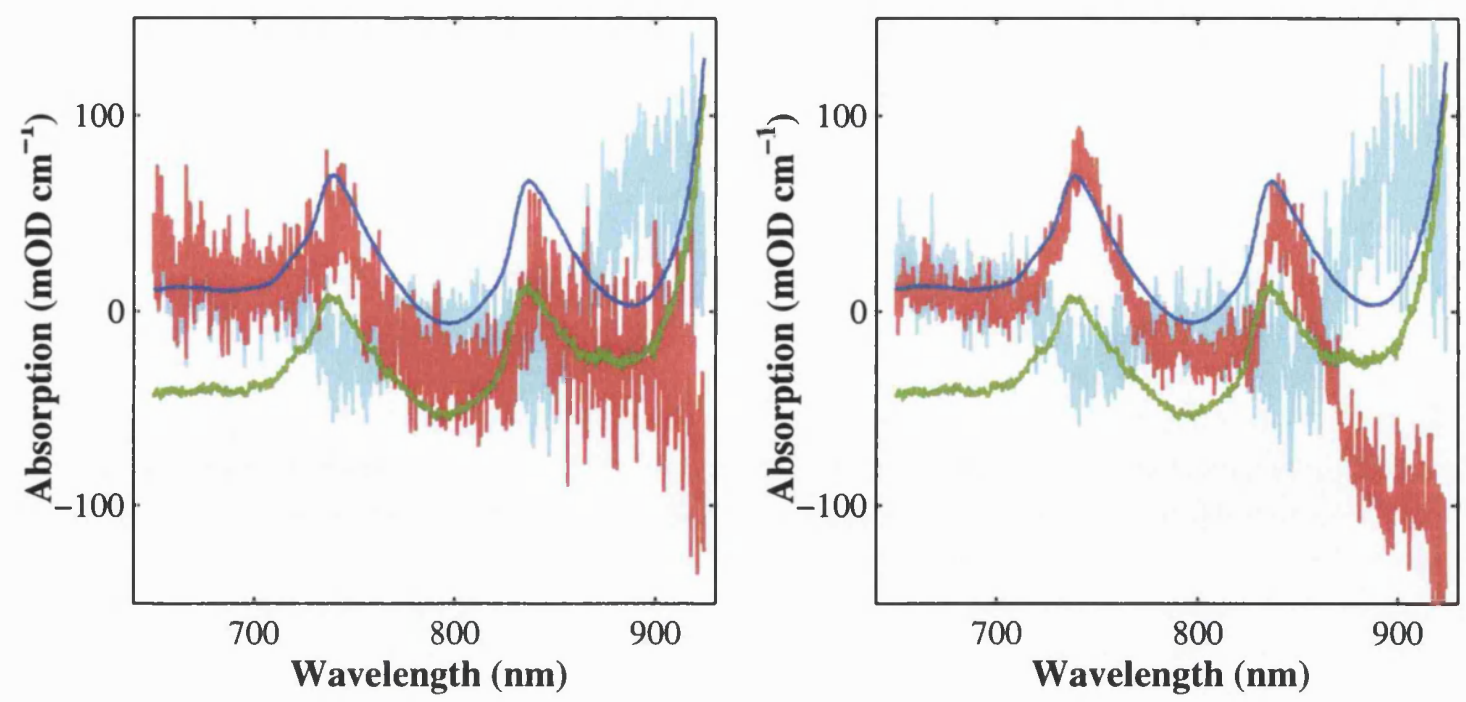

Figure 5.24: Comparison of first four weight and loading vectors from the PLSR temperature calibration of the water absorption spectra. Left: Weight vectors (first- secondthird - fourth ). Right: Loading vectors.

spectral variance described with PCR steadily increases, reaching (effectively) $100 \%$ at ten PCs, whereas with PLSR it doesn't change significantly beyond four or five factors. The converse can be seen for the percentage of variance in the temperature data. The PLSR calibration describes a steadily increasing percentage of the temperature variance with the inclusion of more factors, but with PCR the percentage does not vary much beyond the fourth PC. On inspection of the standard errors it is clear that until the fourth PC or factor the methods do not differ greatly in terms of predictive power. Including the fourth factor with PLSR significantly reduces the SEC to below $0.02{ }^{\circ} \mathrm{C}$, and the error decreases further with more factors. With PCR, however, the SEC does not decrease significantly beyond the inclusion of the fourth $\mathrm{PC}$, remaining greater than $0.032^{\circ} \mathrm{C}$. Although this error seems small in itself one must also take into account the temperature fit residuals over the whole range, as will be discussed shortly.

In order to better understand and interpret the quantitative calibration parameters listed in Table 5.9 it is important to examine the qualitative aspects of the method, for example the loading vectors. As mentioned earlier, in the case of PLSR the loading vectors are usually very similar to the weight vectors. For the calibration of the water temperature spectra the loading and weight vectors for PLSR are virtually identical, as shown in Figure 5.24. The only significant difference is between the third weight and loading vector. In particular they differ in the region $870-900 \mathrm{~nm}$, where the weight vector is constant and the loading vector gives a steadily decreasing value. As can be seen in Figure 5.11 (Section 5.1.3), there is little variation with temperature in this region of the water spectrum, an 


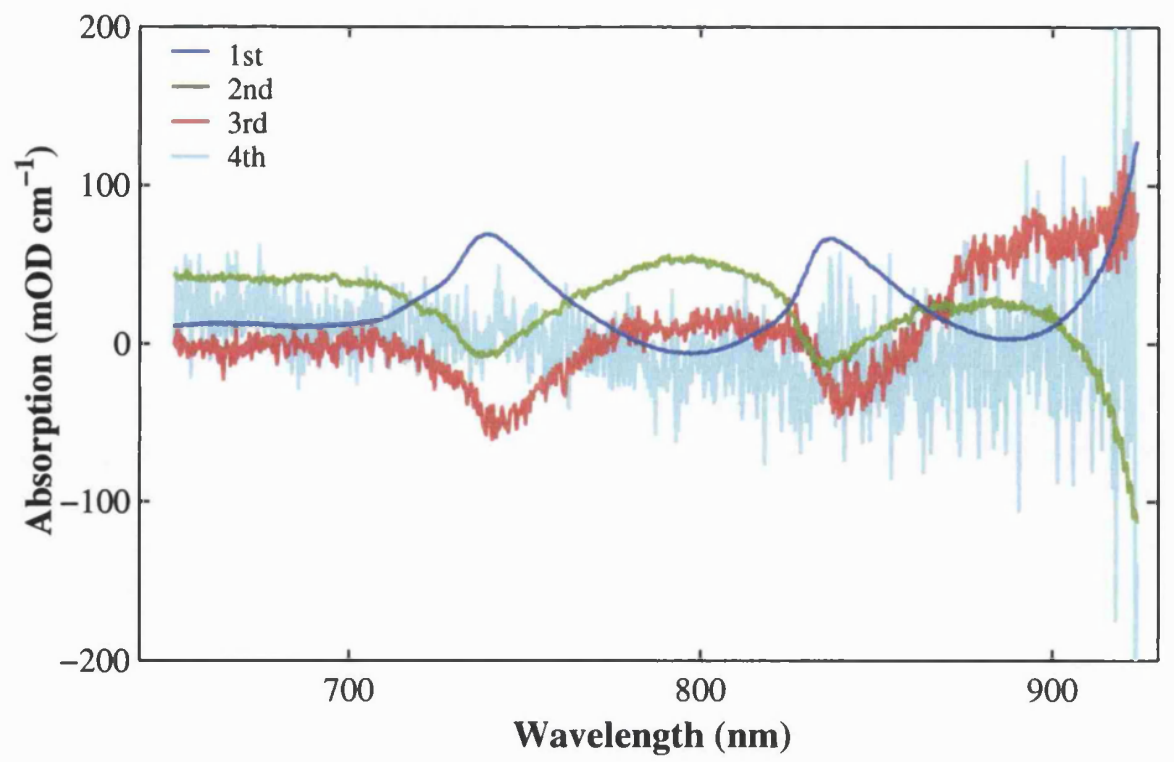

Figure 5.25: The first four loading vectors from the PCR temperature calibration of the water absorption spectra, performed using data set II.

observation that is better reflected by the weight vector for this factor. Beyond the fourth factor there is no obvious structure to the weight or loading vectors; what information they do contain is masked by noise. For comparison the first four PCR loading vectors (obtained from the PCR calibration of data set II) are shown in Figure 5.25. The first three PCR loading vectors are very similar to the PLSR loading vectors, although the second PCR loading vector is the negative of the second PLSR loading vector. (This occurs due to the differences in the way in which the two algorithms calculate the loadings. The score vectors for the second $\mathrm{PC}$ and factor are also reversed in sign relative to each other, such that the combination of score and loading vector for the second PC or factor has the same sign for both methods.) The fourth PCR loading vector, however, contains no useful information about spectral variations, whereas the fourth loading and weight vectors for PLSR show regions of significant variation.

The effect of this difference in loading vectors upon the temperature fit residuals is shown in Figure 5.26. The left-hand graph shows the temperature residuals after the third PC or factor, the right-hand graph shows the residuals including the fourth component. For clarity the PLSR residuals have been offset by $0.2{ }^{\circ} \mathrm{C}$. The residuals for the inclusion of three PCs or factors do not differ greatly for the two methods, showing a certain amount of non-linearity over the whole temperature range. With an additional factor the temperature residuals for the PLSR calibration are significantly improved, removing the non-linearity altogether, as indicated in Table 5.9 by the step reduction in the SEC for 

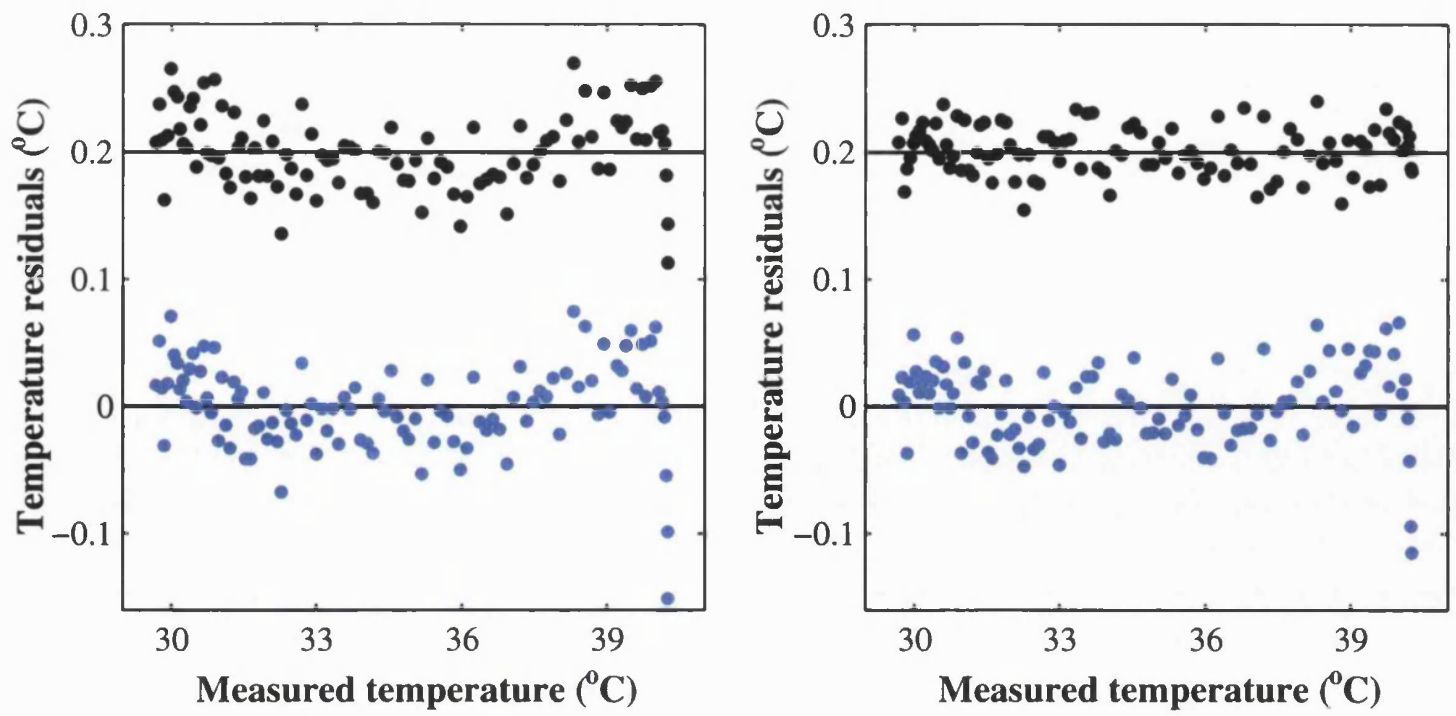

Figure 5.26: Comparison of the temperature residuals determined from the PCR $(\bullet)$ and PLSR $(\bullet)$ calibrations of the water absorption spectra. Left: with 3 PCs or factors. Right: with 4 PCs or factors. The PLSR residuals have been offset by $0.2^{\circ} \mathrm{C}$.

PLSR on moving from three to four factors. The non-linearity in the PCR residuals is not entirely removed by including a fourth PC, nor by the inclusion of further PCs. This is also reflected in Table 5.9, in which the SEC for PCR does not improve beyond three PCs.

Table 5.10 gives the SEP and corresponding $r^{2}$ coefficient for the 'leave-one-out' crossvalidation for both PCR and PLSR. For PCR the SEP did not change significantly beyond the fifth PC and for PLSR the minimum SEP was obtained with four factors, additional factors only increasing the error. For PLSR especially the SEP gives a much clearer estimation of the optimal number of factors to include than the SEC (Martens and Næs, 1991). This is confirmed by the fact that the SEC for PLSR continues to improve beyond the fourth factor (Table 5.9), even when no further useful information can be observed in the loading vectors. The SEP shows that including any more than four factors actually leads to a increase in the standard error, a phenomenon known as 'over-fitting'. The point at which this will occur for the PCR calibration is not so clear, since the SEP for this method continues to increase with the number of PCs.

The PLSR calibration technique has shown itself to be superior to PCR in terms of describing the spectral variation in the water absorption spectrum. In particular the goodness-of-fit of the calibration-estimated temperatures to the measured temperature was improved (albeit at the expense of the spectral fit), as observed in the fit residuals shown in Figure 5.3. Moreover the cross-validation of the calibration data set was seen to yield 


\begin{tabular}{l|ll|ll}
\hline \multirow{2}{*}{$\begin{array}{l}\text { PCs } \\
\text { factors }\end{array}$} & \multicolumn{2}{|c|}{ SEP $\left({ }^{\circ} \mathrm{C}\right)$} & \multicolumn{2}{c}{$\mathrm{r}_{p}^{2}$} \\
\cline { 2 - 5 } & PCR & PLSR & PCR & PLSR \\
\hline 1 & 0.4016 & 0.3969 & 0.9859 & 0.9862 \\
2 & 0.0360 & 0.0359 & 0.9999 & 0.9999 \\
3 & 0.0357 & 0.0345 & 0.9999 & 0.9999 \\
4 & 0.0346 & 0.0316 & 0.9999 & 0.9999 \\
5 & 0.0341 & 0.0330 & 0.9999 & 0.9999 \\
6 & 0.0342 & 0.0334 & 0.9999 & 0.9999 \\
7 & 0.0343 & 0.0336 & 0.9999 & 0.9999 \\
8 & 0.0341 & 0.0338 & 0.9999 & 0.9999 \\
9 & 0.0340 & 0.0341 & 0.9999 & 0.9999 \\
10 & 0.0341 & 0.0344 & 0.9999 & 0.9999 \\
\hline
\end{tabular}

Table 5.10: Comparison of temperature prediction parameters obtained from the application of PCR and PLSR to the NIR water spectra using the cross-validation method, including up to ten PCs or factors in the model

an optimal number of factors for PLSR, which corresponded with the number of loading vectors containing useful information. This was not the case for the cross-validation using PCR. As a result, the PLSR method has been chosen for the development of a further temperature prediction algorithm, to be discussed in Section 7.3. 


\section{Chapter 6}

\section{NIRS Measurements as a Function of Temperature}

Having characterised the water absorption spectrum in Chapter 5, the aim was then to determine if its temperature-dependence could be used to predict temperature from NIRS measurements of scattering media and biological tissue. This chapter describes the various experiments designed for this purpose, discussing the experimental method and results in each case. The prediction of temperature from these measurements will then be discussed in Chapter 7.

As a preliminary study of the effects of temperature on tissue NIRS measurements, Section 6.1 describes the ex vivo measurement of chicken muscle in the temperature range $23-43^{\circ} \mathrm{C}$. Although these results are not subsequently employed for testing temperature prediction algorithms, they indicate the likely effects of changes in temperature on the optical properties of tissue. Section 6.2 describes NIRS measurements of the adult human forearm (by a co-worker) with decreasing temperature to approximately $26^{\circ} \mathrm{C}$. The results of this study are used extensively to develop and test the temperature prediction algorithms of Chapter 7. In Section 6.3 the temperature-dependence of a tissue-like phantom is studied using multi-distance (spatially-resolved) measurements. These data will be used in the final temperature prediction algorithm, discussed in Section 7.3. 


\subsection{Chicken Muscle}

\subsubsection{Experimental Design and Method}

As a preliminary study of the effects of temperature on tissue attenuation, spectral measurements were made from $650-805 \mathrm{~nm}$ on skinless chicken breast over the temperature range $23-43^{\circ} \mathrm{C}$. As for the water measurements described in Section 5.1.2, the experimental set-up was placed inside a neonatal incubator. The light source and spectrograph-CCD system described in Section 3.2 were also employed. The muscle tissue was wrapped in a clear polyethylene film ('cling film'), to avoid loss of water on heating, and placed inside a rectangular container made from $1 \mathrm{~cm}$ thick black plastic. The dimensions of the container were $8 \mathrm{~cm}$ high by $8 \mathrm{~cm}$ wide by $3 \mathrm{~cm}$ deep. The fibres and temperature sensors were inserted into holes made in the sides of the container, such that the source and detecting fibres were opposite one another just under $3 \mathrm{~cm}$ apart (in a transmission geometry), and ensuring that the sensors were firmly embedded in the tissue but did not obscure the field of view of the fibres. The fibres were clamped in place to avoid any movement artifacts and both fibres and sensors were additionally secured by black masking tape, which also shielded the container from ambient light.

Prior to measuring the tissue, a reference measurement in air was made (see Section 3.1 , the light intensity attenuated by a suitable amount using a 4 OD neutral density filter. The fibres were then placed in good contact with the muscle tissue and the tissue was heated in the incubator from an ambient temperature of $23^{\circ} \mathrm{C}$ to approximately $43^{\circ} \mathrm{C}$, during which time spectral measurements were made. The 600 lines $\mathrm{mm}^{-1}$ grating was used with a spectrograph slit width of $50 \mu \mathrm{m}$, giving a spectral resolution of 0.31 $\mathrm{nm}$. The spectral acquisition time for the measurements was set such that the intensity acquired was approximately 100,000 electron counts. Data collection was terminated at the maximum temperature $\left(\sim 43^{\circ} \mathrm{C}\right)$ and the acquisition time re-adjusted to once again obtain a reasonable signal. The temperature of the incubator was then lowered to $30^{\circ} \mathrm{C}$ and data acquisition was resumed during cooling of the tissue. The entire experiment (heating and cooling stages) lasted approximately 8 hours, after which the total loss in mass of the muscle tissue was determined to be less than $0.5 \%$.

\subsubsection{Results}

The spectral data from both heating and cooling phases was exported from the acquisition software and, as with the water data (Section 5.1), was averaged such that the maximum 


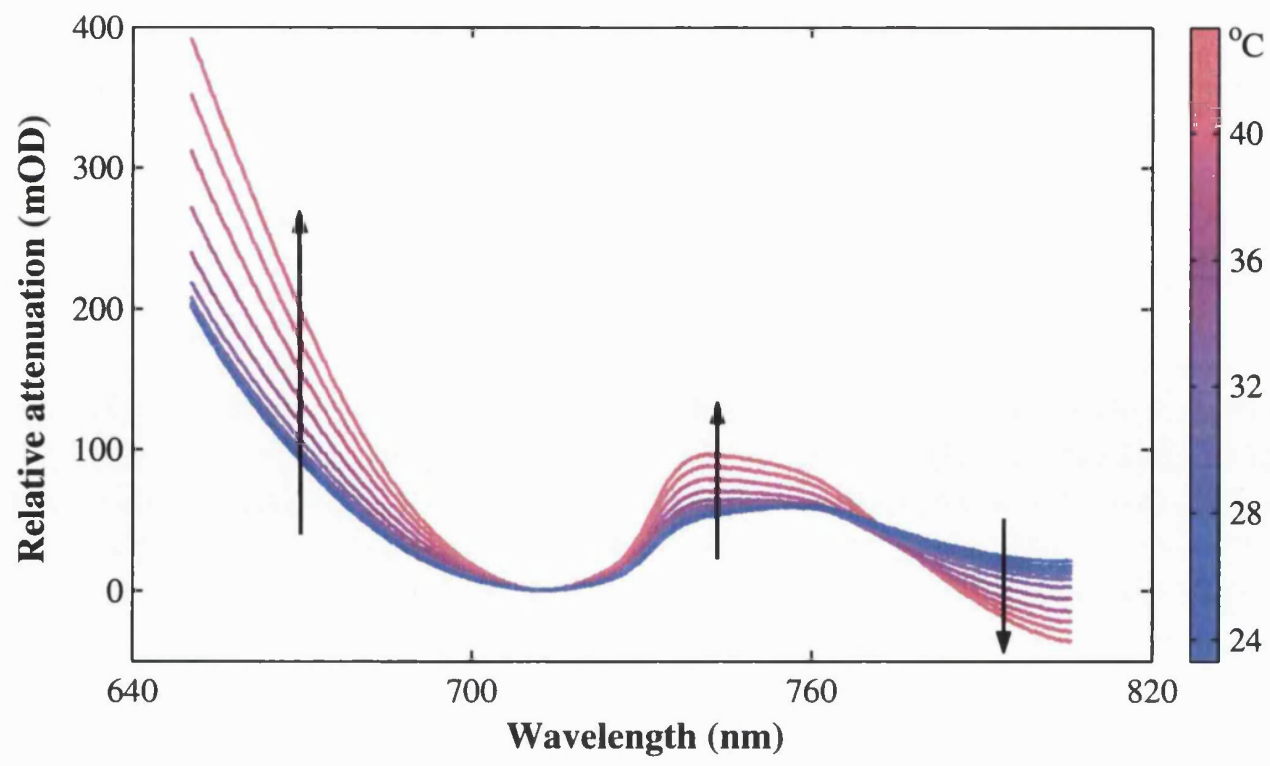

Figure 6.1: Relative changes in attenuation, with respect to $710 \mathrm{~nm}$, with heating of chicken muscle tissue between 23 and $43^{\circ} \mathrm{C}$ - arrows show the direction of increasing temperature.

temperature change between spectra was $0.05^{\circ} \mathrm{C}$. The attenuation at all wavelengths was seen to increase significantly during tissue heating. Moreover, changes in the wavelengthdependence of attenuation also occurred with temperature. Since the baseline attenuation offset increased considerably during heating $(\sim 0.9$ OD), the spectra were (arbitrarily) zeroed at $710 \mathrm{~nm}$, the wavelength at which the attenuation in the first spectrum was at a minimum, in order to show changes in the shape of the spectra with temperature more clearly. Figure 6.1 displays the relative attenuation spectra of the chicken muscle as a function of increasing temperature. The arrows indicate the direction of increasing temperature, which varies over the spectral range. (It should be noted that the apparent decrease in attenuation with temperature in the region above $\sim 770 \mathrm{~nm}$ is not an actual decrease in the measured attenuation but a decrease with respect to that at $710 \mathrm{~nm}$.) As the temperature increased the slope of the spectra appeared to increase, indicated by the opposing directions of the arrows at the ends of the spectra. However, the region from 720 to $770 \mathrm{~nm}$ increased in amplitude with temperature, relative to the zeroed wavelength, which could imply an increased absorption in this range (see Section 6.1.3 for further discussion).

As mentioned in Section 6.1.1, due to the large increases in attenuation upon heating the tissue, the acquisition time per spectrum had to be increased for the second stage of the experiment in order to maintain a reasonable signal. The changes in attenuation upon cooling the tissue were not as great as for the heating phase. Moreover, attenuation 


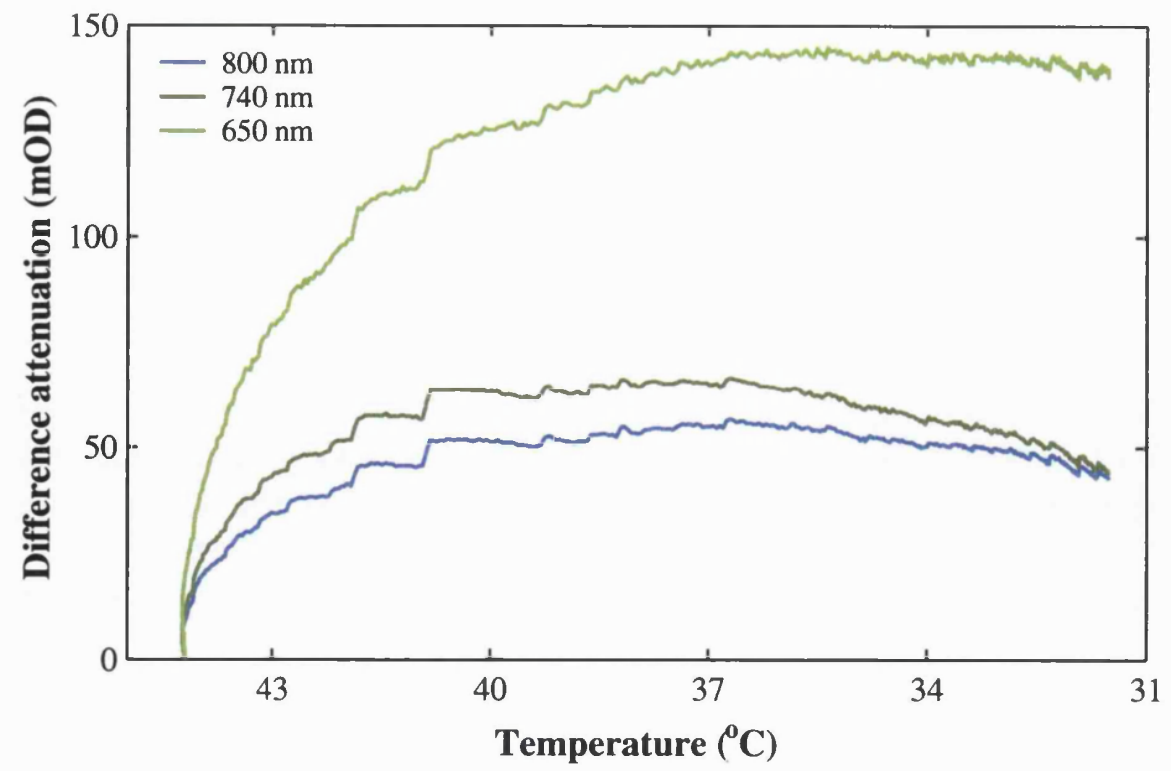

Figure 6.2: Difference attenuation at three wavelengths during cooling of chicken muscle tissue, displayed as a function of decreasing tissue temperature. Attenuation is seen to increase at all wavelengths until about $37^{\circ} \mathrm{C}$, after which it decreases at some wavelengths, the greatest changes being at $740 \mathrm{~nm}$.

continued to increase for some time after cooling began. Figure 6.2 attempts to best demonstrate the effects of tissue cooling by displaying the changes in attenuation (at three wavelengths), with respect to that measured at the maximum temperature, as a function of decreasing tissue temperature. It can be seen that at each wavelength the attenuation continues to increase until approximately $37^{\circ} \mathrm{C}$. Below this temperature the attenuation at $650 \mathrm{~nm}$ does not appear to change significantly, whilst at 740 and $800 \mathrm{~nm}$ the attenuation starts to decrease again with temperature, the decrease being the greatest at $740 \mathrm{~nm}$.

\subsubsection{Discussion}

The results displayed in Figures 6.1 and 6.2 are consistent with the findings of other workers, discussed in Sections 4.3.1.3 and 4.3.2. Jaywant et al (1993) observed an immediate increase in the scattering coefficient of bovine muscle when heating from $30^{\circ} \mathrm{C}$, the effect being greater at shorter wavelengths (as seen with the chicken muscle attenuation spectra in Figure 6.1). They attributed the observation to the denaturation of proteins within the tissue, resulting in a greater number of small particle scatterers, which scatter more efficiently at shorter wavelengths. The increase in attenuation caused by a scattering increase will depend on the underlying absorption coefficient at that wavelength. For a given increase in $\mu_{s}^{\prime}$, the change in attenuation will be greater at higher values of $\mu_{a}$. 
The absorption coefficient of chicken muscle was measured by Marquez et al (1998), who indicated contributions from haemoglobin and possibly myoglobin. Below approximately $700 \mathrm{~nm}$ the absorption coefficient increases steadily due to absorption by $\mathrm{Hb}$. Hence, the greater increase in attenuation of the chicken muscle at shorter wavelengths (due to a larger relative increase in scattering here) will be further exacerbated by the higher $\mu_{a}$ values at these wavelengths.

As shown in Figure 6.1, the relative attenuation change with temperature between 770 and $805 \mathrm{~nm}$ is smaller than at $710 \mathrm{~nm}$ (the wavelength at which the spectra were zeroed), possibly due to a combination of a smaller scattering change and a lower $\mu_{a}$. Between 710 and $770 \mathrm{~nm}$, however, the relative attenuation increase is greater than at $710 \mathrm{~nm}$. In this region there will be contributions to the absorption by muscle from haemoglobin and water. Therefore, although the scattering increase may be slightly larger at $710 \mathrm{~nm}$, the increased absorption in the $710-770 \mathrm{~nm}$ region results in a larger attenuation change with temperature. Interestingly, the change in attenuation at $740 \mathrm{~nm}$ is significantly greater than that at $760 \mathrm{~nm}$. One would expect that with very similar changes in scattering likely at wavelengths in such close proximity, the greatest change in attenuation would be seen at the wavelength with the larger underlying absorption, which in this case should be 760 $\mathrm{nm}$ due to the larger absorption by the $\mathrm{Hb}$ band at $760 \mathrm{~nm}$ than the water band at 740 $\mathrm{nm}$ (Marquez et al, 1998). This is not the case, however, the implication being that the absorption at $740 \mathrm{~nm}$ is also increasing with temperature, relative to $760 \mathrm{~nm}$. Therefore, it is possible that the attenuation measurements of chicken muscle shown here indicate the increase in the absorption by tissue water at $740 \mathrm{~nm}$ with temperature.

Another factor regarding the attenuation changes with temperature at $760 \mathrm{~nm}$ is the possible conversion of haemoglobin to methaemoglobin. As mentioned in Section 4.3.1.3, denaturation of proteins due to heating can result in the oxidisation of haem groups within the haemoglobin molecule, producing methaemoglobin which, in comparison to the $\mathrm{Hb}$ spectrum, has a greater absorption at $650 \mathrm{~nm}$ and a smaller absorption at $760 \mathrm{~nm}$ (Cope, 1991). This could further help to explain the large increases in attenuation at $650 \mathrm{~nm}$, i.e. due to increased absorption by methaemoglobin, and the smaller increase in attenuation at $760 \mathrm{~nm}$ relative to $740 \mathrm{~nm}$, due to the reduction in absorption by $\mathrm{Hb}$.

As the tissue was cooled the attenuation continued to increase for some time, as shown in Figure 6.2. It is possible that the temperature of the tissue measured by the sensors was not the same as that sampled by the light, particularly close to the source fibre. On inspection of the tissue after the experiment, the tissue in the area surrounding the 
source fibre appeared especially whitish in colour, indicating thermal damage had occurred. One explanation for the increase in attenuation with tissue cooling, therefore, is that the temperature of the tissue sampled by the light was still increasing after the peripheral tissue temperature measured by the sensors had decreased. Various authors have successfully modelled the thermally-induced denaturation of tissue proteins as a first-order Arrhenius process (Agah et al, 1996; Skinner et al, 2000), in which the rate of thermal damage at a given temperature is characterised by the $e^{-1}$ variation in the optical coefficients, in particular $\mu_{s}^{\prime}$. The fraction of the total change in $\mu_{s}^{\prime}$ occurring after a time $t$ is modelled as

$$
\frac{\mu_{s}^{\prime}(t)}{\mu_{s \max }^{\prime}}=\exp (-k t)
$$

where $\mu_{s \max }^{\prime}$ is the maximum change in $\mu_{s}^{\prime}$ due to complete thermal damage and $k$ is the rate constant for the process, given as a function of temperature $\mathrm{T}$ by the Arrhenius equation

$$
k(\mathrm{~T})=A \exp \left(-\frac{E_{a}}{\mathrm{RT}}\right)
$$

where $A$ here is the Arrhenius factor (a measure of the frequency of collisions between molecules), $E_{a}$ is the activation energy for the process and $\mathrm{R}$ is the universal gas constant. In general the lower the temperature the slower the rate of thermal damage, and for a given temperature the longer the time of heating the greater the damage, or change in $\mu_{s}^{\prime}$. In the current study of muscle tissue it is therefore possible that thermal damage was still occurring after the cooling phase began, and only stopped at such a time when the tissue temperature happened to be approximately $37^{\circ} \mathrm{C}$. Using the values for the Arrhenius factor and the activation energy obtained for rat prostrate (Skinner et al, 2000), heating tissue for 4 hours at $37^{\circ} \mathrm{C}$ would result in approximately $97 \%$ thermal coagulation, or $97 \%$ of the total change in $\mu_{s}^{\prime}$. Although not the same tissue, this may explain why the attenuation measured in the chicken muscle, the tissue having been at a temperature greater than $37^{\circ} \mathrm{C}$ for about 4 hours, reached a maximum at the arbitrary temperature of $37^{\circ} \mathrm{C}$, because at this point the maximal thermal damage had occurred.

At $650 \mathrm{~nm}$ the attenuation did not change significantly as the tissue was cooled below $37^{\circ} \mathrm{C}$, as shown in Figure 6.2. It is likely that thermal damage to the tissue induced by the heating produced an irreversible change in the scattering coefficient (see Section 3.3. Hence, if there are no significant changes in absorption on cooling, the measured attenuation will remain constant as the temperature drops. There is a significant decrease, however, in the attenuation around the $740 \mathrm{~nm}$ region from about $37^{\circ} \mathrm{C}$ onwards. An 
explanation for the increase in attenuation around this region on heating was given as the increase in absorption by water. Similarly, the decrease in attenuation on cooling, most prominent in the $740 \mathrm{~nm}$ region, could be due to the temperature-induced decrease in the water absorption coefficient. Note, however, that since the scattering coefficient is likely to be permanently altered by the heating process one could not expect to regain the original shape of the spectra on cooling.

The results of this experiment indicate that it is possible to observe changes in the optical properties of tissue with temperature using the experimental method described. Moreover, the results suggest that both scattering and absorption changes are occurring, based on the effect of temperature on the spectral-dependence of the measurements. In this experiment the tissue was heated above $40^{\circ} \mathrm{C}$ which appeared to cause an irreversible change in the structure of the tissue, due to protein denaturation, and hence the scattering coefficient. As such the cooling phase was not directly comparable to the heating phase in terms of attenuation changes. The main clinical application of this project is to determine in vivo tissue temperature during hypothermia, i.e. below normal body temperature, hence permanent thermal damage to tissues should not be a factor. From the observations made, it is clear that the experiment was not perhaps the best model for the clinical situation, therefore these preliminary results have not been further analysed.

\subsection{Human Forearm}

\subsubsection{Experimental Design and Method}

To investigate the effect of temperature on in vivo spectroscopic measurements of tissue, attenuation spectra measured on the adult human forearm by a co-worker were employed. The aim of the original study used NIRS to investigate changes in oxygen metabolism in resting human skeletal muscle as a function of temperature (Binzoni et al, 1999). The experimental set-up is shown in Figure 6.3.

In these experiments the volunteer's arm was cooled from 37 to $26^{\circ} \mathrm{C}$ by immersing it in a water bath (shown in Figure 6.3) and lowering the water temperature. The temperature of the arm was determined using a DBT sensor (Deep Body Thermometers Ltd., Cambridge UK), placed on the inner forearm. This non-invasive technique is based on the principle of zero heat flow, previously described in Section 4.1. Briefly, the assumption is that if the heat flow across an insulating probe attached to the skin surface is zero, there must exist a region of uniform temperature in the tissue beneath the skin, the temperature 


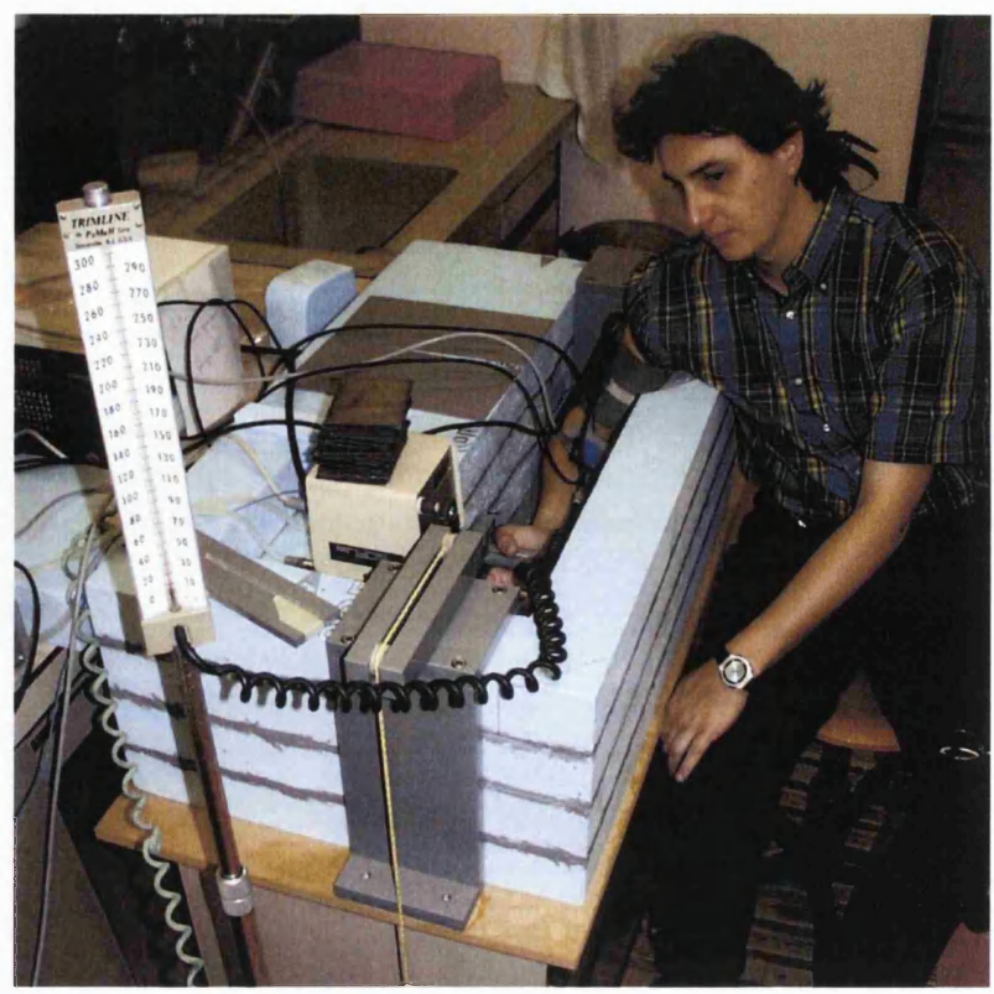

Figure 6.3: Experimental set-up for in vivo measurements of the adult forearm as a function of temperature (Binzoni et al, 1999). Spectral measurements were made during cooling of the arm from 37 to $26^{\circ} \mathrm{C}$.

of which is determined by sensors within the probe. The DBT probe used in this study covered an area of $6 \mathrm{~cm}^{2}$ on the surface of the skin. This enabled a region of tissue beneath the probe approximately $3 \mathrm{~cm}^{2}$ in area to reach a uniform temperature to a depth of about $1.5 \mathrm{~cm}$.

The probe was modified to incorporate two optical fibres with end-mounted prisms (optodes) in contact with the skin surface and separated from one another by $3 \mathrm{~cm}$ in a reflection geometry. The source fibre was coupled to a white light source (Oriel) and the detector fibre to a spectrograph-CCD system (Wright Instruments; Instruments SA), both of which were described in Section 3.2. Spectra were acquired over the range 670-980 nm, with a 300 grooves $\mathrm{mm}^{-1}$ grating and a spectrograph slit width of $400 \mu \mathrm{m}$, giving a spectral resolution of $5 \mathrm{~nm}$. Prior to measurements of the forearm a reference measurement through air was made by placing the probe over the port-hole of a custom-made integrating sphere. The attenuation spectra displayed in the next section are referenced to this measurement and therefore have an arbitrary baseline. The spectral measurements were acquired continuously whilst cooling the forearm, the entire experiment lasting approximately 2 hours. 


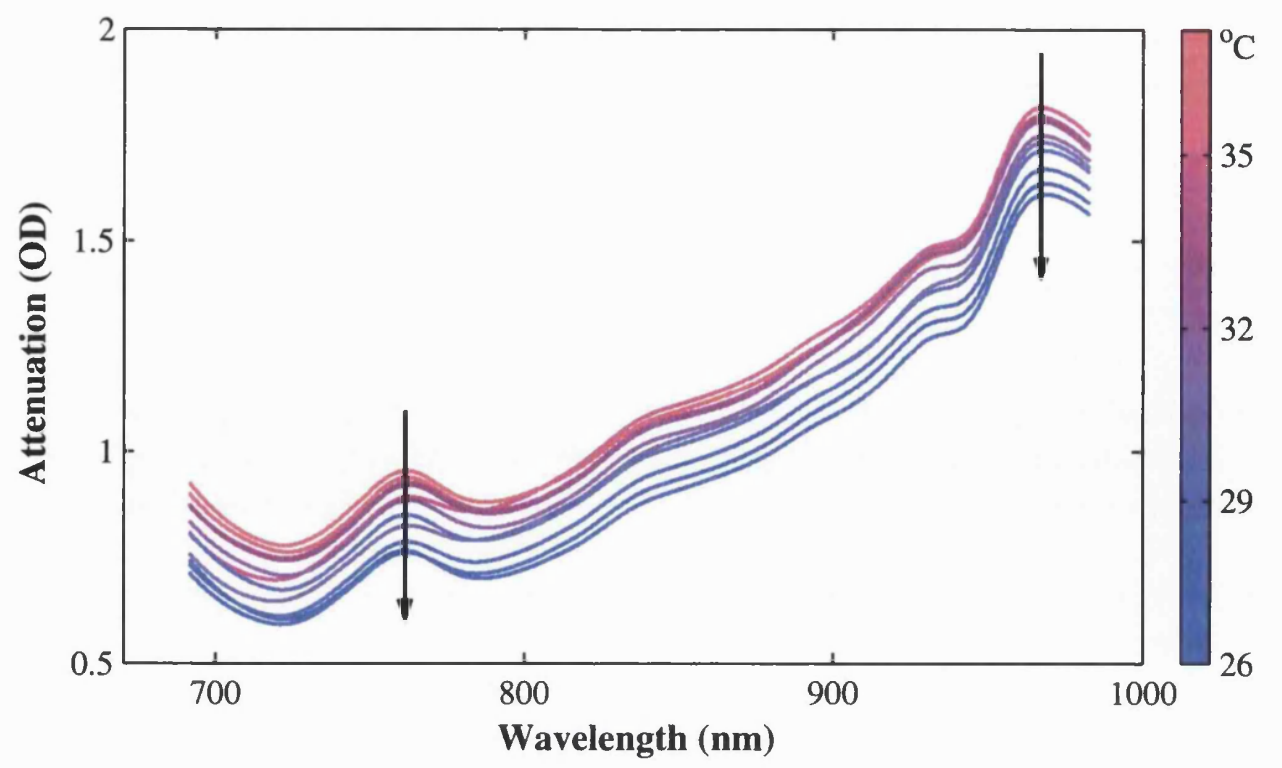

Figure 6.4: Attenuation spectra measured during cooling of the adult forearm from 37$26^{\circ} \mathrm{C}$ (Binzoni et al, 1999) - arrows show the direction of decreasing temperature.

\subsubsection{Results and Discussion}

Figure 6.4 shows the spectral measurements of the adult forearm as a function of temperature between 37 and $26^{\circ} \mathrm{C}$, the arrow showing the direction of decreasing temperature. The spectra were averaged such that the maximum change in temperature between contiguous spectra was $0.1^{\circ} \mathrm{C}$. As with the water data the forearm spectra were smoothed by a filter for clearer display. The obvious features of the spectra are the water band at approximately $970 \mathrm{~nm}$ and the $\mathrm{Hb}$ peak at around $760 \mathrm{~nm}$. The $840 \mathrm{~nm}$ water band is just visible, and the shoulder appearing at approximately $930 \mathrm{~nm}$ is due to absorption by lipids, present in adipose tissue in the subdermal region.

The changes in the attenuation spectra with temperature are best represented by the final difference attenuation spectrum, i.e. the last spectrum subtracted from the first spectrum, as shown in Figure 6.5 (the negative values indicate that attenuation decreased with temperature at all wavelengths). Changes in attenuation with temperature will be due to a combination of changes in absorption and scattering in the tissue, and because of the non-linearity of the relationship between attenuation and $\mu_{a}$ and $\mu_{s}^{\prime}$, will depend on the magnitude of the initial optical coefficients prior to the change. In general, the changes in attenuation will be greatest where the optical coefficients are the largest. Moreover, the change in attenuation for a given $\mu_{a}$ change is greater at smaller initial values of $\mu_{a}$ and larger values of $\mu_{s}^{\prime}$, and vice versa for $\mu_{s}^{\prime}$ (see Section 2.3).

As discussed in Section 4.3.2, Yeh et al (2001) observed a reversible decrease in the 


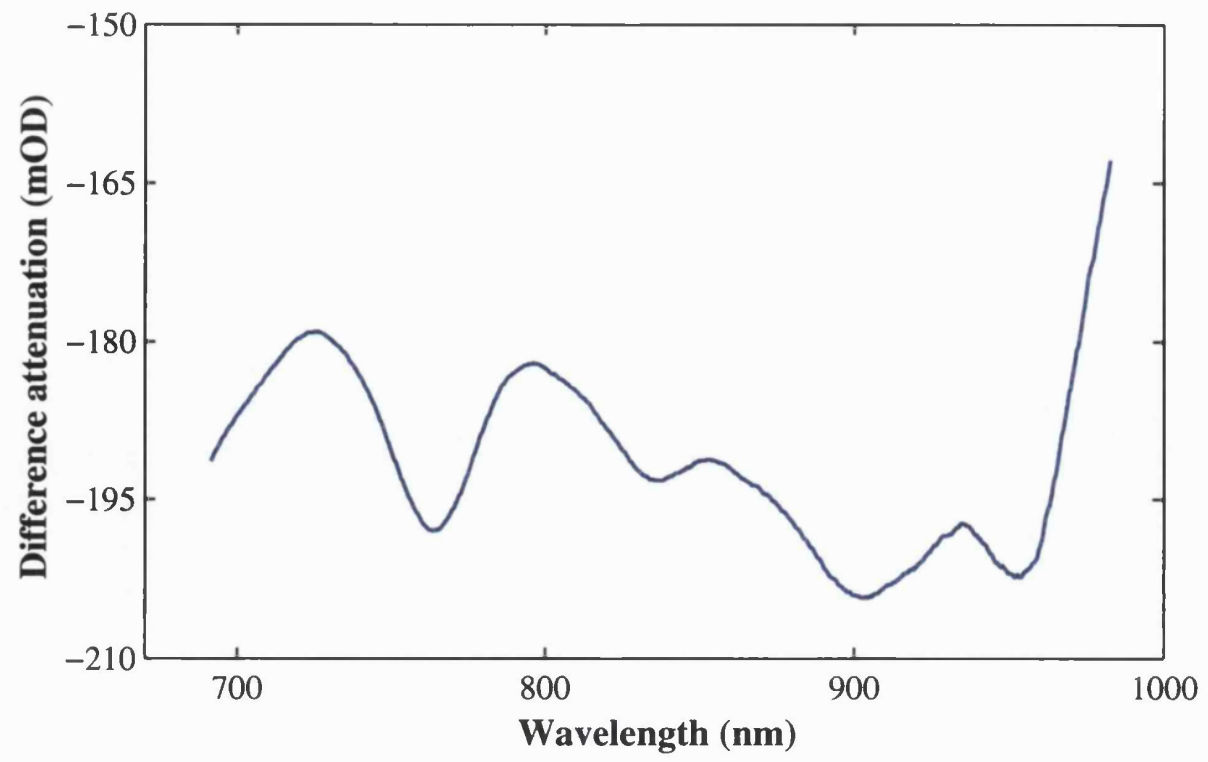

Figure 6.5: Final difference attenuation spectrum of the adult forearm cooled from 37 to $26^{\circ} \mathrm{C}$ (Binzoni et al, 1999) - the negative values indicate that attenuation has decreased with temperature.

transport scattering coefficient of the adult forearm with decreasing temperature in the range $25-41^{\circ} \mathrm{C}$, the magnitude of which was greater at shorter wavelengths. However, the source-detector fibre separation was much smaller than in the present study $(<0.2$ $\mathrm{cm}$ as opposed to $3 \mathrm{~cm}$ ), and Yeh et al assumed that the region probed by the light encompassed only the dermis and contained no adipose tissue, the latter of which both they and Laufer et al (1998) determined to have a negative temperature coefficient for $\mu_{s}^{\prime}$. In a study of the effect of adipose tissue on NIRS measurements, van Beekvelt et al (2001) determined the skin and adipose tissue thickness to be $0.25 \pm 0.08 \mathrm{~cm}$ in the adult male forearm. Based on the average optical coefficients determined for the adult forearm in a separate time-resolved study (Matcher et al, 1997), the average photon penetration depth (defined in Section 3.1) will be approximately $0.6 \mathrm{~cm}$ (at $800 \mathrm{~nm}$ ) for a source-detector separation of $3 \mathrm{~cm}$. (It should be noted, though, that this average penetration depth will change with temperature as $\mu_{a}$ and $\mu_{s}^{\prime}$ change.) This suggests that the the light will additionally penetrate the muscle tissue beneath the subdermal fat layer, and that the temperature coefficient of $\mu_{s}^{\prime}$ for the forearm may well be governed by that for muscle tissue, which, since both tissue types are rich in protein, is likely to be the same as that in dermis (positive) for the reasons discussed by Laufer et al (1998) and Yeh et al (2001). Hence, the transport scattering coefficient can be expected to decrease with decreasing temperature in the present study of the adult forearm (Binzoni et al, 1999).

Changes in absorption with temperature will contain contributions from water, 
haemoglobin and possibly lipid. As temperature decreases the water absorption coefficient decreases around the 740, 840 and $970 \mathrm{~nm}$ bands (see Figure 5.11). The absorption band at around $840 \mathrm{~nm}$ can be distinguished from the other spectral features in Figure 6.5. Conversely, the absorption coefficients of $\mathrm{Hb}$ and $\mathrm{HbO}_{2}$ are known to increase with decreasing temperature (see Section 4.3.1.2). The haemoglobin oxygen saturation increases with decreasing temperature, however, due to a reduction in the demand for oxygen (see Section 4.3.1.3). This effect will decrease the concentration of $\mathrm{Hb}$ in favour of $\mathrm{HbO}_{2}$, as seen in Figure 6.5 by the noticeable difference attenuation in the region of the $760 \mathrm{~nm} \mathrm{Hb}$ band, which may well overlap the decrease in the $740 \mathrm{~nm}$ water absorption band. Furthermore, the total concentration of haemoglobin in the region probed could be decreasing due to the physiological responses to cooling of the arm (see Section 7.2 for further discussion). The large difference attenuation at $950 \mathrm{~nm}$ will contain a significant contribution from the decrease in absorption by water with temperature. However, compared to the maximum temperature coefficient of water absorption at $962 \mathrm{~nm}$ (Figure 5.11), it is slightly shifted in wavelength, due to both a smaller underlying $\mu_{s}^{\prime}$ and smaller changes in $\mu_{s}^{\prime}$ with temperature at $962 \mathrm{~nm}$ relative to $950 \mathrm{~nm}$ (Matcher et al, 1997; Yeh et al, 2001). The latter also explains why the change in attenuation at $840 \mathrm{~nm}$ is greater than expected, relative to that at $962 \mathrm{~nm}$, based on the temperature coefficients of water absorption alone (Figure 5.11). The results of this study will be discussed in more detail during the development of the temperature prediction algorithms given in Sections 7.2.

\subsection{Tissue Phantom}

A tissue phantom is a turbid medium (solid or liquid) used to mimic the optical properties of biological tissue. The final NIRS measurements performed as a function of temperature during this project were made on such a phantom, the methodology and results of which will be discussed in this section. The important difference between these and the measurements described in the previous two sections is that the phantom measurements were spatially-resolved, i.e. more than one source-detector fibre separation was employed simultaneously. The purpose of using multiple source-detector separations, as will be discussed in Section 7.3, is to determine both $\mu_{a}$ and $\mu_{s}^{\prime}$ from one set of measurements. Since the (time-independent) reflectance of light from a scattering medium (defined in Section $2.3)$ is a function of $\mu_{a}, \mu_{s}^{\prime}$ and the source-detector separation, one should in theory be able to determine the absorption and transport scattering coefficients in the least-squares 
sense from a minimum of three spatially-resolved measurements. The phantom measurements will be used to develop and test a final temperature prediction algorithm, detailed in Section 7.3.

\subsubsection{Experimental Design and Method}

The tissue phantom consisted of Intralipid-10\% $\%^{\mathrm{TM}}$ (Pharmacia-Upjohn, Milton Keynes $\mathrm{UK})$, isotonic saline and a $1 \mathrm{M}$ copper sulphate $\left(\mathrm{CuSO}_{4}\right)$ solution at concentrations (by volume) of $10,89.7$ and $0.3 \%$ respectively. Intralipid- $10 \%^{\mathrm{TM}}$ is a lipid-based emulsion (used clinically as an intravenous nutrient) which is often used as an optical phantom for tissue spectroscopy studies (Nakai et al, 1997; Mourant et al, 1997). Isotonic saline (see Section 5.2.2) was used for the bulk of the phantom to more closely approximate the constituents of biological tissue. Copper sulphate has a broad, featureless NIR absorption and was used to increase the absorption of the phantom to approximately that of tissue. The specific absorption coefficient of the $\mathrm{CuSO}_{4}$ solution (Figure 6.6) in the region 650-925 $\mathrm{nm}$ was determined by measuring the spectrum of a known concentration of $\mathrm{CuSO}_{4}$, referenced to that of pure water, in a glass cuvette, and was found to be $13.0 \mathrm{OD} \mathrm{cm}^{-1} \mathrm{M}^{-1}$ at $800 \mathrm{~nm}$. The concentrations of the scatterer (Intralipid) and the absorber (predominantly $\mathrm{CuSO}_{4}$ ) were chosen such that the optical properties of the phantom were around 0.043 and $4.34 \mathrm{OD} \mathrm{cm}^{-1}$ for $\mu_{a}$ and $\mu_{s}^{\prime}$ respectively, some typical values for biological tissue (see Section 3.3). The transport scattering coefficient of the Intralipid was determined from the equation of van Staveren et al (1991) for a $1 \%$ Intralipid solution (i.e. $10 \%$ of Intralipid-

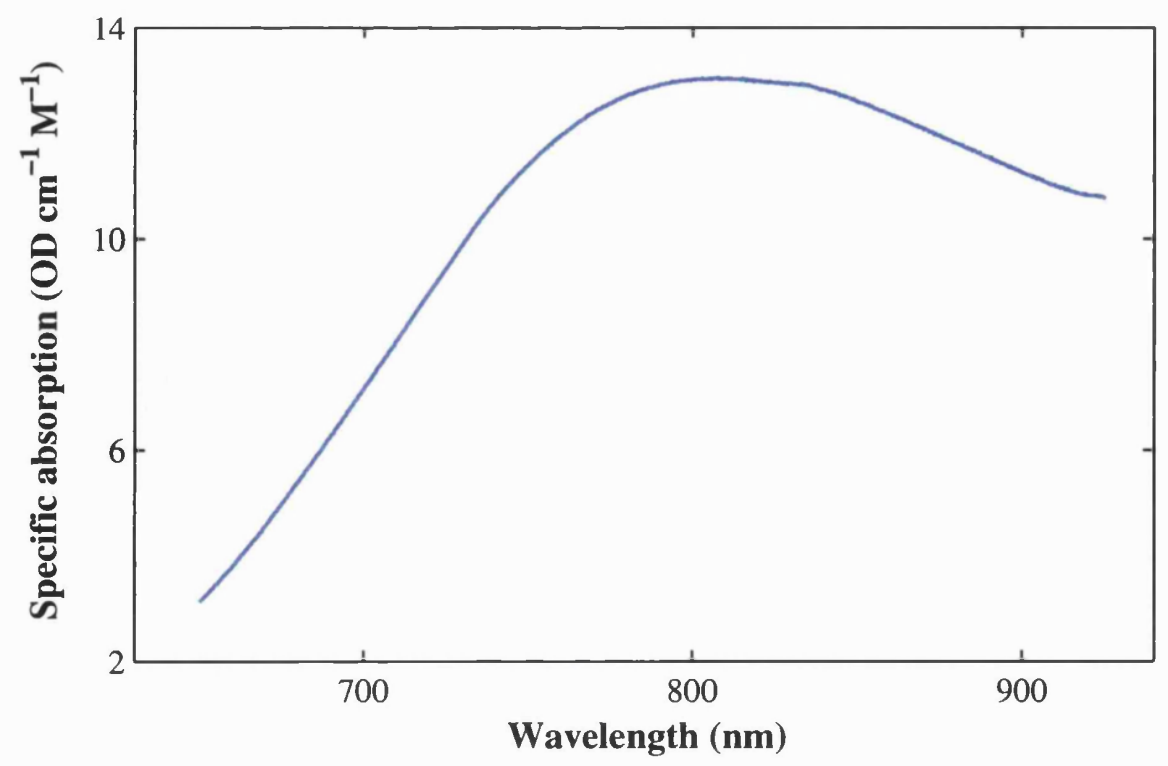

Figure 6.6: The specific absorption spectrum of copper sulphate in the region $650-925 \mathrm{~nm}$ 
$10 \%)$. The absorption and transport scattering coefficients of the phantom as a whole are shown in Figure 6.10 in Section 6.3.2, estimated for a temperature of $37^{\circ} \mathrm{C}$. The liquid phantom, a total volume of $\sim 3.5$ litres, was held in a plastic container approximately 24.5 $\mathrm{cm}$ square and $10 \mathrm{~cm}$ deep.

The detecting fibre consists of three fibre bundles, each $2 \mathrm{~mm}$ in diameter, set into a rectangular brass ferrule at the common end. The bundles from each fibre are aligned in an array of 'windows', approximately $1.7 \mathrm{~mm}^{2}$ in area, in the face of the ferrule. The three windows are evenly-spaced within a vertical column of total height $\sim 8 \mathrm{~mm}$. As explained in Section 3.2, the light from a detecting fibre is first collimated then focussed on to the spectrograph entrance slit by a system of lenses. For the spatially-resolved measurements the focussing lens used for previous measurements was replaced by a lens of longer focal length, in order to achieve a smaller, more sharply focussed image of the light from each fibre bundle on the slit. The light from the fibres is then incident on three clearly defined regions of the CCD chip, each approximately 65 pixels (i.e. $1.7 \mathrm{~mm}$ ) in height. The spectral measurement for each fibre position was obtained by binning vertically over each of the three regions separately (see Section 3.2). However, because of the large increase in attenuation with separation from the source fibre $(\sim 1$ OD per $\mathrm{cm})$, the number of pixels binned in each region was altered to account for the limited dynamic range of the CCD detector ( 1.3 OD). Each of the $2 \mathrm{~mm}$ fibre bundles are terminated (at the distal end) in a short piece of nylon and the whole bundle is loosely surrounded by a protective PVC sheath of outer diameter $5 \mathrm{~mm}$. To prevent the phantom liquid wicking up the fibres a thin piece of latex rubber was fastened over the end of each bundle, secured in place with a small o-ring.

The source and detector fibres were inserted into the phantom medium a few millimeters below the surface, supported by a black plastic holder, with source-detector fibre separations of $1.8,2.7$ and $3.6 \mathrm{~cm}$. The temperature sensors were fixed to the bottom of the holder with cable ties and masking tape, one near the source fibre and one close to the furthest detecting fibre, i.e. the sensors were separated by almost $4 \mathrm{~cm}$. The difference in temperature recorded by the two sensors was always less than $0.3^{\circ} \mathrm{C}$, hence the temperature of the phantom was taken to be the average of the two sensor readings. The entire set-up (phantom container and fibres) was placed into the neonatal incubator described in Section 5.1.1. To prevent the constituents of the phantom settling during the experiment, and to avoid temperature gradients within the liquid, the phantom was stirred continuously with a peristaltic pump. 
To reduce the time taken to reach the initial temperature $\left(\sim 41^{\circ} \mathrm{C}\right)$ the phantom was placed into a microwave oven for about 10 minutes on low heat, raising its temperature to approximately $40^{\circ} \mathrm{C}$. A polyethylene film ('cling film') was stretched over the top of the phantom container (with a small weight placed in the centre) to prevent water evaporation, which could alter the scattering properties of the phantom. The phantom and its container were weighed prior to heating, and again at the end of the experiment some five hours later. The total volume loss was approximately $1 \%$, although some of this was due to residual liquid in the pump circuit, which will not significantly alter $\mu_{s}^{\prime}$ of the phantom. After heating in the oven the phantom was placed into the incubator and its temperature allowed to stabilise at around $41^{\circ} \mathrm{C}$.

The spatially-resolved measurements of the tissue phantom were performed in the range $600-925 \mathrm{~nm}$ between 41 and $34^{\circ} \mathrm{C}$. An air reference measurement was first made by positioning the three fibre bundles (out of the fibre holder) at the port-hole of an integrating sphere (Section 3.2) illuminated by the source fibre. The acquisition time was $600 \mathrm{~ms}$ and the spectrograph slit width was $100 \mu \mathrm{m}$, with a spectral resolution of $1.2 \mathrm{~nm}$. For each detecting region the reference spectrum was set for the corresponding fibre bundle. During the reference measurement the aperture on the front of the QTH light source was reduced in size in order to attenuate the light input to the integrating sphere. For the phantom measurements the aperture was re-adjusted to its maximum size, allowing a greater input intensity into the phantom. Thus no adjustment of the acquisition time or slit width was required for the phantom measurements relative to the reference measurement, although the difference in input intensities precluded an 'absolute' measurement of the phantom attenuation (relative to air). As will be discussed in Section 7.3, however, the important aspect of these measurements is the change in the shape of the spectrum with temperature, not the absolute values. In order to place the fibres and temperature sensors in contact with the phantom a hole was cut in the film over the container and the holder inserted. The edges of the film were then taped to the fibre holder at such an angle that water evaporating from the phantom would run back into the container. The same procedure was performed for the pump input and output tubes. The spectral measurements began as soon as the fibres were in place, with the incubator closed and shielded from ambient light as before. The incubator temperature was set to $20^{\circ} \mathrm{C}$ after about 2 minutes and measurements were made during cooling of the phantom over approximately 4 hours. Due to the large thermal mass of the phantom the liquid only cooled to about $34^{\circ} \mathrm{C}$ during this time, compared to $\sim 30^{\circ} \mathrm{C}$ for the water absorption 
measurements (Section 5.1).

\subsubsection{Results}

The phantom spectra were exported from the acquisition software, averaging over $0.1^{\circ} \mathrm{C}$ (i.e. $140 \mathrm{~s}$ or 58 spectra), and imported into MATLAB ${ }^{\circledR}$. Figure 6.7 shows the phantom attenuation spectra measured at the three source-detector separations at an initial temperature of $40.8^{\circ} \mathrm{C}$. Because the attenuation baseline is arbitrary (due to the nonabsolute measurement), the spectra have been zeroed at approximately $650 \mathrm{~nm}$ to show the relative differences between them, and are also smoothed by a filter for clearer display. As predicted by the analytical solution to the diffusion approximation of reflectance from a semi-infinite turbid medium (Section 2.3), the attenuation measured at the surface of the medium increases with distance from the source. At all three separations the water absorption bands at 740 and $840 \mathrm{~nm}$ are evident. The band around $740 \mathrm{~nm}$ is quite broad, indicating an overlap between the water and lipid absorption bands in this region. The lipid in Intralipid is soybean oil, a neutral fat (or triglyceride, see Section 2.1.3) that is liquid at room temperature. The absorption spectrum of soybean oil (43005, Sigma-Aldrich) was measured in the temperature range $40-28^{\circ} \mathrm{C}$, using the method described in Section 5.1.2.3 for the pure water measurement, and is displayed at $37^{\circ} \mathrm{C}$ in Figure 6.8. The absorption spectrum exhibited no obvious temperature-dependence, although scattering

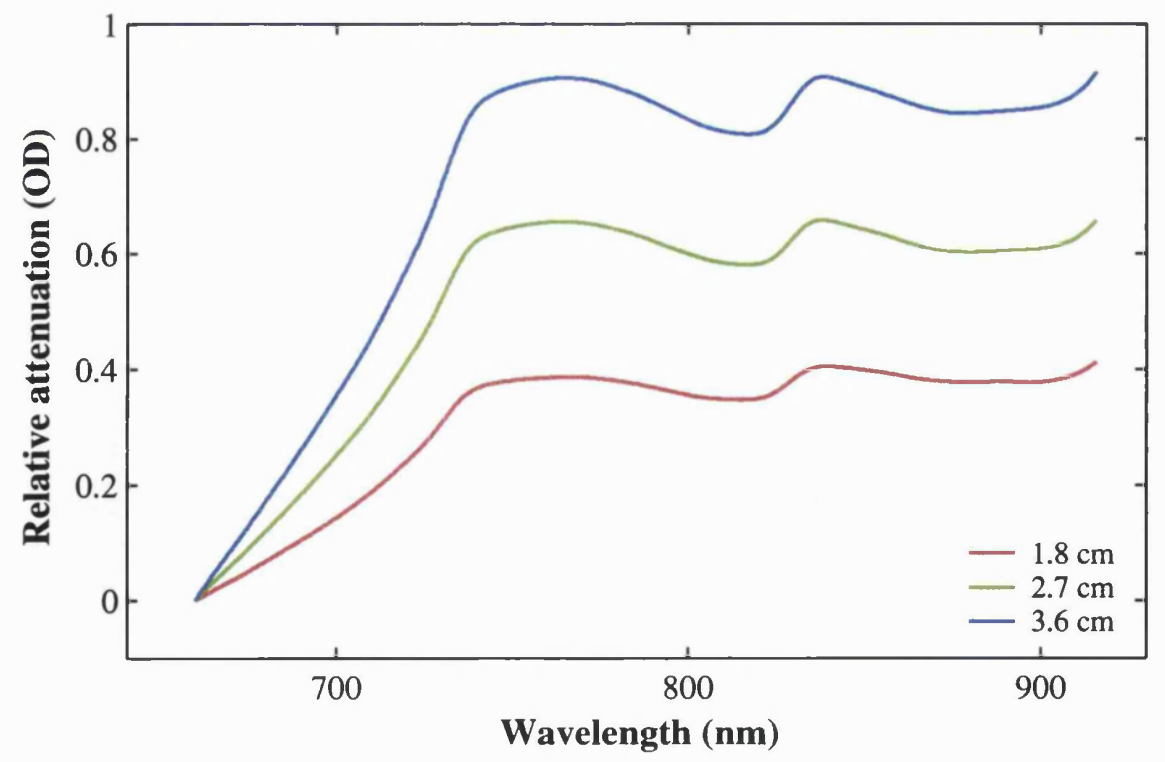

Figure 6.7: Spatially-resolved attenuation spectra of tissue phantom measured at $40.8^{\circ} \mathrm{C}$ using multi-distance fibre with source-detector separations of $1.8,2.7$ and $3.6 \mathrm{~cm}$. The spectra are zeroed at $650 \mathrm{~nm}$ to show relative attenuation and are smoothed for clearer display. 


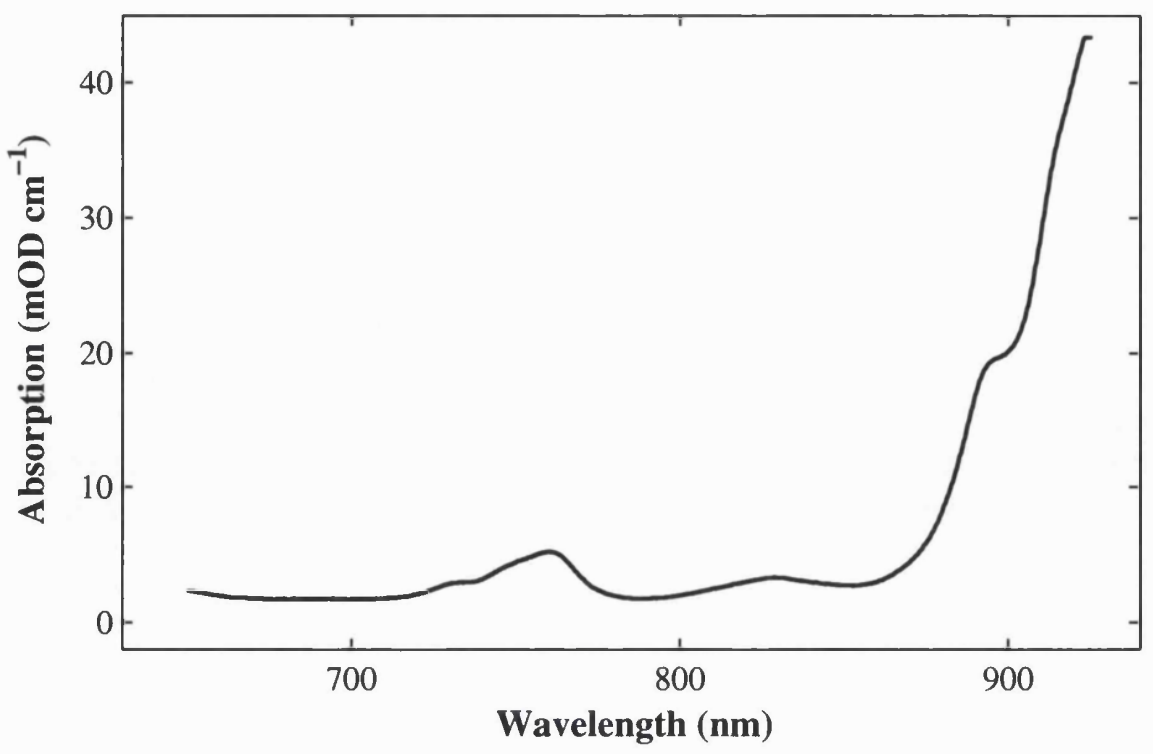

Figure 6.8: Absorption spectrum of soybean oil (constituent of Intralipid-10\% ${ }^{\mathrm{TM}}$ ) measured at $37^{\circ} \mathrm{C}$ in the range $650-925 \mathrm{~nm}$

in the oil did appear to change, indicated by an increasing linear offset (greater at shorter wavelengths) between the spectra with decreasing temperature.

Figure 6.9 shows the final difference attenuation spectra measured at the three separations in the temperature range $40.8-33.8^{\circ} \mathrm{C}$. As mentioned previously in Section 6.2.2, changes in attenuation with temperature will depend on both the changes in $\mu_{a}$ and $\mu_{s}^{\prime}$ and their initial values. Moreover, the relative effect of changes in the optical properties

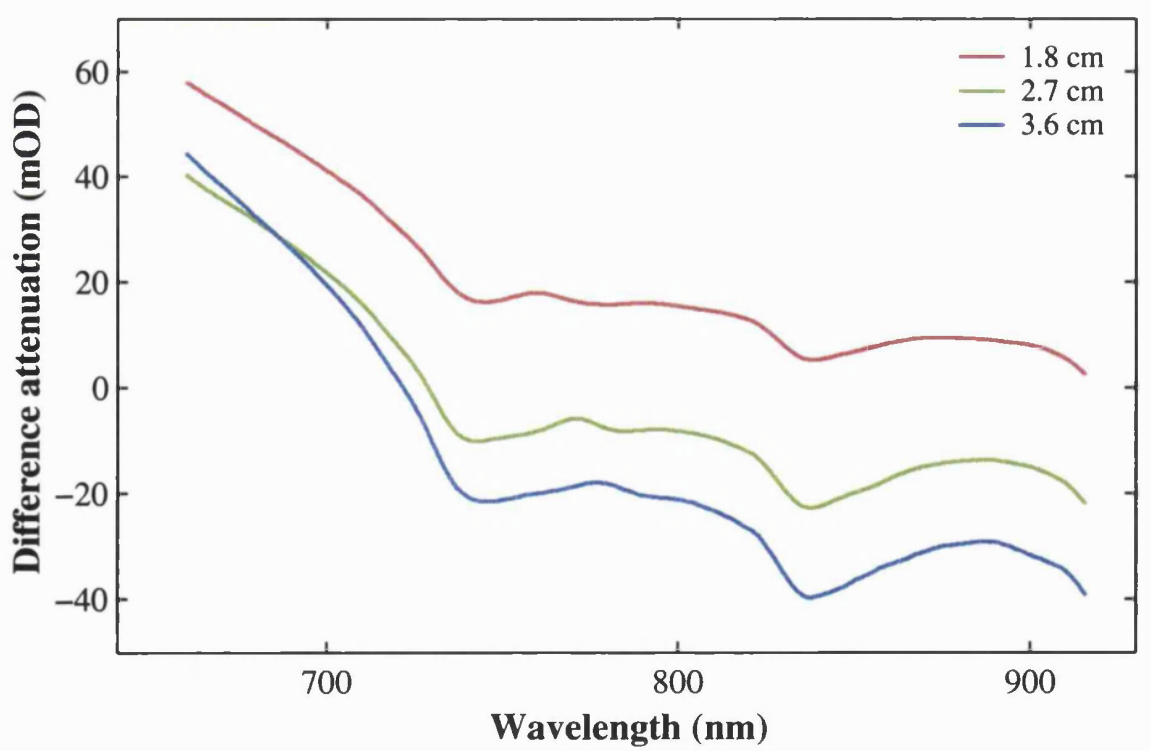

Figure 6.9: Final difference attenuation spectra of spatially-resolved phantom measurements over the temperature range $40.8-33.8^{\circ} \mathrm{C}$. Positive values indicate an increase in attenuation with decreasing temperature, negative values indicate a decrease. 
with temperature on measurements of attenuation will alter with the source-detector separation. These two phenomena, the generic changes in attenuation with temperature and the effects at different source-detector separations, will be discussed in Section 6.3.3.

\subsubsection{Discussion}

In order to interpret and discuss the magnitude and wavelength-dependence of the attenuation changes with temperature, the optical coefficients of the phantom were estimated at a mid-point temperature for the measurements of $37^{\circ} \mathrm{C}$. The absorption spectrum of the phantom will contain contributions from $\mathrm{CuSO}_{4}$, saline, and lipid and water in the Intralipid. The total spectrum at $37^{\circ} \mathrm{C}$ was estimated using the following concentrations (as they occur in the phantom) of the component spectra: $89.7 \%$ saline, $9 \%$ pure water (essentially $90 \%$ of the Intralipid is water), $1 \%$ lipid and $0.3 \% \mathrm{CuSO}_{4}$. The measurement of the absorption spectrum of isotonic saline around $37^{\circ} \mathrm{C}$ was described in Section 5.2.2, as was that for pure water in Section 5.1. The absorption spectrum of the lipid at $37^{\circ} \mathrm{C}$ is displayed in Figure 6.8. The $\mathrm{CuSO}_{4}$ spectrum, displayed in Figure 6.6, was measured at room temperature, although its temperature-dependence will be due to water and a correction was made to bring the reference for this measurement (water) to $37^{\circ} \mathrm{C}$. The transport scattering spectrum was estimated by using the formula of van Staveren et al (1991), determined from measurements of collimated transmittance through Intralipid$10 \%^{\mathrm{TM}}$ at several discrete wavelengths in the visible and NIR regions. However, these
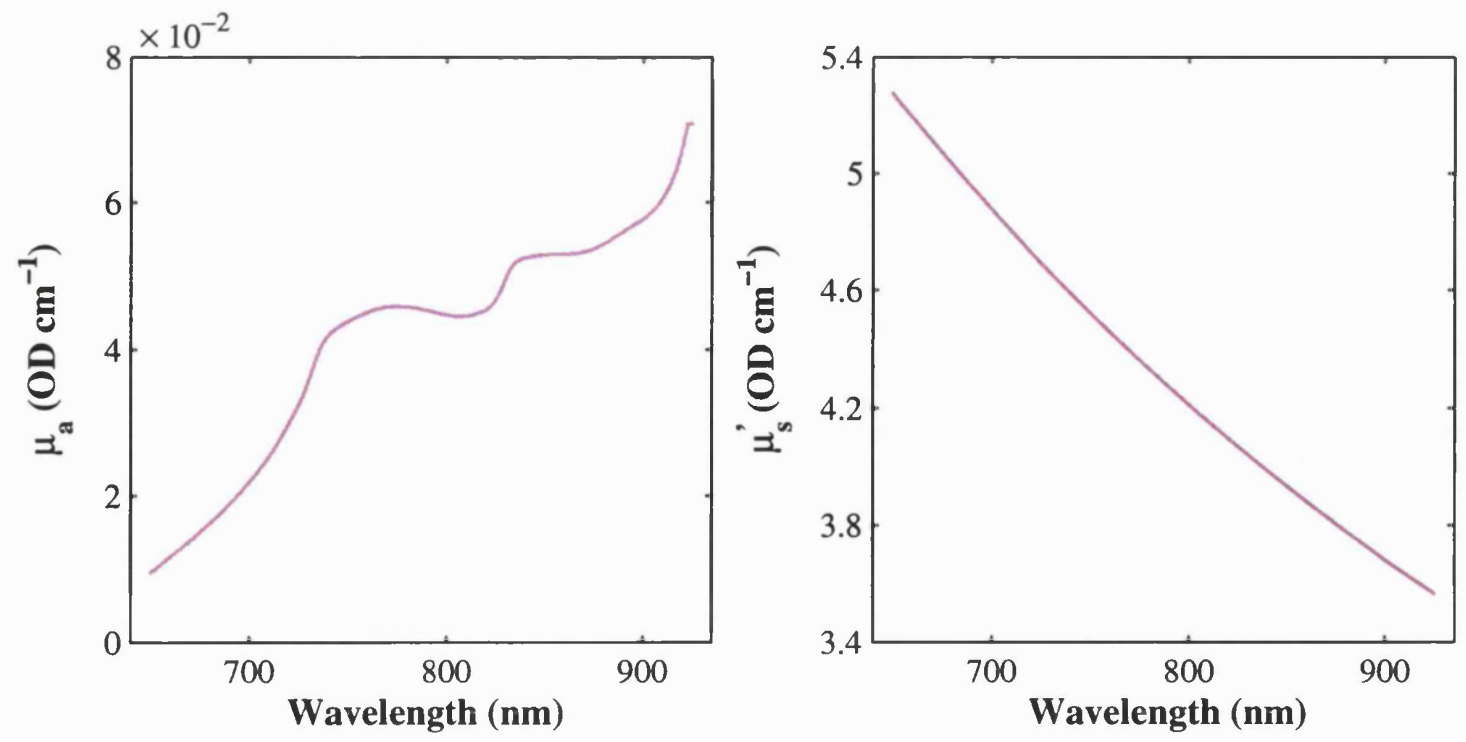

Figure 6.10: The absorption and transport scattering spectra of the tissue phantom in the range $650-925 \mathrm{~nm}$, estimated for a temperature of $37^{\circ} \mathrm{C}$, see text for details. 
measurements were made at room temperature, and no direct measurements of the scattering coefficient of Intralipid at $37^{\circ} \mathrm{C}$ could be found in the literature. The transport scattering spectrum at $37^{\circ} \mathrm{C}$ was therefore estimated, based on the results of Chance et al (1995). Using a linear approximation for the spatially-resolved reflectance of a semiinfinite medium (see Section 7.3 for further details), Chance et al (1995) determined a change in $\mu_{s}^{\prime}$ of a $1 \%$ Intralipid solution (i.e. $10 \%$ of Intralipid- $10 \%$ ) of $-0.18 \%{ }^{\circ} \mathrm{C}^{-1}$ in the temperature range $20-38^{\circ} \mathrm{C}$ at $850 \mathrm{~nm}$. Assuming room temperature to be $22^{\circ} \mathrm{C}$, the transport scattering coefficient at $37^{\circ} \mathrm{C}$ can be estimated to be $2.69 \%$ lower than at room temperature. Although the change in the transport scattering coefficient with temperature may be wavelength-dependent (Yeh et al, 2001), the above correction was applied to the $\mu_{s}^{\prime}$ spectrum for a $1 \%$ Intralipid solution (determined by the equation of van Staveren et $a l)$ at all wavelengths, since no additional information about the temperature-dependence of Intralipid optical properties could be found. The absorption and transport scattering coefficients of the tissue phantom in the range $650-925 \mathrm{~nm}$, estimated at a temperature of $37^{\circ} \mathrm{C}$, are displayed in Figure 6.10.

The temperature-dependence of the tissue phantom $\mu_{a}$ will be due to that of water, i.e. as temperature decreases, absorption by the phantom in the region of the water absorption bands will also decrease. The temperature-dependence of the transport scattering coefficient will be dominated by that of the lipid, which, as demonstrated by Chance et al (1995) in Intralipid and by others in their studies of human tissue (Laufer et al, 1998; Yeh et al, 2001), increases with decreasing temperature. The overall effect on attenuation at a given wavelength and source-detector separation will depend on the temperature coefficients of $\mu_{a}$ and $\mu_{s}^{\prime}$ and their underlying values. The difference attenuation spectra displayed in Figure 6.9, at the various source-detector separations, have some common features. Firstly, the relative changes with temperature at shorter wavelengths are more positive than those at longer wavelengths. Increases in $\mu_{s}^{\prime}$ with decreasing temperature will result in positive changes in attenuation. For a given change in $\mu_{s}^{\prime}$ the change in attenuation will tend to be greater at wavelengths for which $\mu_{s}^{\prime}$ is small and $\mu_{a}$ is large, i.e. at longer wavelengths (see Figure 6.10). However, the decrease in temperature produces a negative slope with respect to wavelength in the difference attenuation spectra (larger changes at shorter wavelengths). In the region below $700 \mathrm{~nm}$ there is very little change in the absorption coefficient of water with temperature (see Figure 5.11), which suggests that the negative slope of the difference attenuation (at least in this region) is dominated by the temperature coefficient of $\mu_{s}^{\prime}$, which must therefore be greater at shorter wavelengths 
to oppose the larger $\mu_{s}$ and smaller $\mu_{a}$ values. The effects on attenuation of a decrease in absorption with decreasing temperature can also be observed superimposed on this slope. Absorption effects are dominant around 740 and $840 \mathrm{~nm}$, as seen by a relative decrease in the difference attenuation in these regions. The more negative difference attenuation at $840 \mathrm{~nm}$ relative to that at $900 \mathrm{~nm}$ can be explained by the fact that the decrease in absorption at $840 \mathrm{~nm}$ outweighs the smaller temperature coefficient of $\mu_{s}^{\prime}$ at $900 \mathrm{~nm}$, where the temperature coefficient of water absorption is practically zero (see Figure 5.11). This effect is further exacerbated by the fact that the underlying $\mu_{a}$ is smaller and the $\mu_{s}^{\prime}$ is greater at $840 \mathrm{~nm}$ compared to $900 \mathrm{~nm}$.

When the source-detector separation is much larger than the transport mean free path $\left(\mu_{t}^{\prime-1}\right)$, the diffuse reflectance from the surface of a scattering medium is essentially linear with increasing source-detector separation, the slope dominated by the effective attenuation coefficient $\mu_{\text {eff }}$ (see Section 2.3). For a given change in the optical properties, i.e. at a given wavelength, the changes in attenuation are greater at larger source-detector separations, which explains the differences in the difference attenuation spectra shown in Figure 6.9. Moreover, as the separation increases the changes in attenuation depend more strongly on changes in $\mu_{a}$ relative to changes in $\mu_{s}^{\prime}$. As discussed in Section 5.1.3, any potential drift in the intensity of the light source was not corrected for using the set-up described, hence its contribution to changes in attenuation with temperature cannot be ruled out. Due to the precautions taken, however, the drift is likely to be small (see Section 5.1.3), and should not in any case affect the wavelength-dependence of the changes with temperature. The results of the tissue phantom experiment are discussed in more detail in Section 7.3. 


\section{Chapter 7}

\section{NIRS Tissue Temperature Prediction Algorithms}

This chapter describes the various algorithms developed for predicting temperature from NIRS measurements of attenuation, using the measurements described in Chapter 6. Common to all algorithms is the use of the temperature calibrations of the water absorption spectrum, detailed in Chapter 5, to predict temperature from the absorption spectra underlying the measurements of attenuation. The algorithms differ, however, in the manner in which they determine absorption from attenuation.

In Section 7.1 the technique of second derivative spectroscopy in its application to determine tissue chromophore concentrations, by approximating a linear relationship between attenuation and absorption, is discussed. The potential of this method to predict temperature directly from attenuation measurements is investigated. Section 7.2 describes a technique for reconstructing the underlying absorption spectra of temperature-dependent attenuation measurements using a non-linear Newton-Raphson optimisation routine. Temperature is then predicted by applying the calibration of the water absorption spectrum to the reconstructed absorption coefficients. The algorithm described in Section 7.3 uses a linear approximation of the diffusion theory solution for spatially-resolved reflectance to determine both absorption and scattering coefficients from multi-distance attenuation measurements of the tissue phantom described in Section 6.3. Again, temperature is predicted using the temperature calibrations discussed in Section 5.3. 


\subsection{Algorithm I: Linear SDS Method}

The development of the first temperature prediction algorithm is based on the technique of second derivative spectroscopy. As discussed in Section 3.1, in the presence of scatter the absorption of a medium is no longer proportional to the attenuation of light measured through the medium, and scattering losses will contribute an unknown, wavelengthdependent baseline offset to the attenuation spectrum. These baseline offsets can be eliminated, however, by differentiating with respect to wavelength. The first derivative will remove a constant, wavelength-independent baseline and the second derivative will remove a first-order baseline. Derivative spectroscopy is a technique used in a wide variety of spectroscopic applications, for example, to reduce the effects of instrumental drift, or to resolve overlapping spectral bands (Fearn, 1999). This section discusses the application of second derivative spectroscopy (SDS) to NIRS measurements of tissue attenuation to determine the concentrations at which the absorbing tissue chromophores are present. A brief introduction to the theory and the method by which the derivative spectrum is obtained is given, then the use of SDS to determine tissue chromophore concentrations is described and applied to simulated tissue attenuation spectra. Finally, Algorithm I investigates the ability of SDS to predict temperature directly from measurements of tissue attenuation as a function of temperature, using the in vivo measurements of the adult forearm described in Section 6.2 and the PCR calibration of the water absorption spectrum described in Section 5.3.5.

\subsubsection{Theory}

The second derivative of attenuation $A\left(\mu_{a}, \mu_{s}^{\prime}\right)$ with respect to wavelength is given by

$$
\begin{aligned}
\frac{\mathrm{d}^{2} A}{\mathrm{~d} \lambda^{2}}= & \frac{\partial A}{\partial \mu_{a}} \frac{\mathrm{d}^{2} \mu_{a}}{\mathrm{~d} \lambda^{2}}+\frac{\mathrm{d} \mu_{a}}{\mathrm{~d} \lambda}\left(\frac{\mathrm{d} \mu_{a}}{\mathrm{~d} \lambda} \frac{\partial^{2} A}{\partial^{2} \mu_{a}}+\frac{\mathrm{d} \mu_{s}^{\prime}}{\mathrm{d} \lambda} \frac{\partial^{2} A}{\partial \mu_{s}^{\prime} \partial \mu_{a}}\right) \\
& +\frac{\partial A}{\partial \mu_{a}} \frac{\mathrm{d}^{2} \mu_{s}^{\prime}}{\mathrm{d} \lambda^{2}}+\frac{\mathrm{d} \mu_{s}^{\prime}}{\mathrm{d} \lambda}\left(\frac{\mathrm{d} \mu_{a}}{\mathrm{~d} \lambda} \frac{\partial^{2} A}{\partial \mu_{a} \partial \mu_{s}^{\prime}}+\frac{\mathrm{d} \mu_{s}^{\prime}}{\mathrm{d} \lambda} \frac{\partial^{2} A}{\partial \mu_{s}^{\prime 2}}\right)
\end{aligned}
$$

In its application to NIR tissue spectroscopy to determine chromophore concentrations, the SDS technique assumes the first term on the right hand side in Equation 7.1 to be dominant, such that the second derivative of attenuation with respect to wavelength can be approximated as

$$
\frac{\mathrm{d}^{2} A}{\mathrm{~d} \lambda^{2}} \approx \frac{\partial A}{\partial \mu_{a}} \frac{\mathrm{d}^{2} \mu_{a}}{\mathrm{~d} \lambda^{2}}
$$




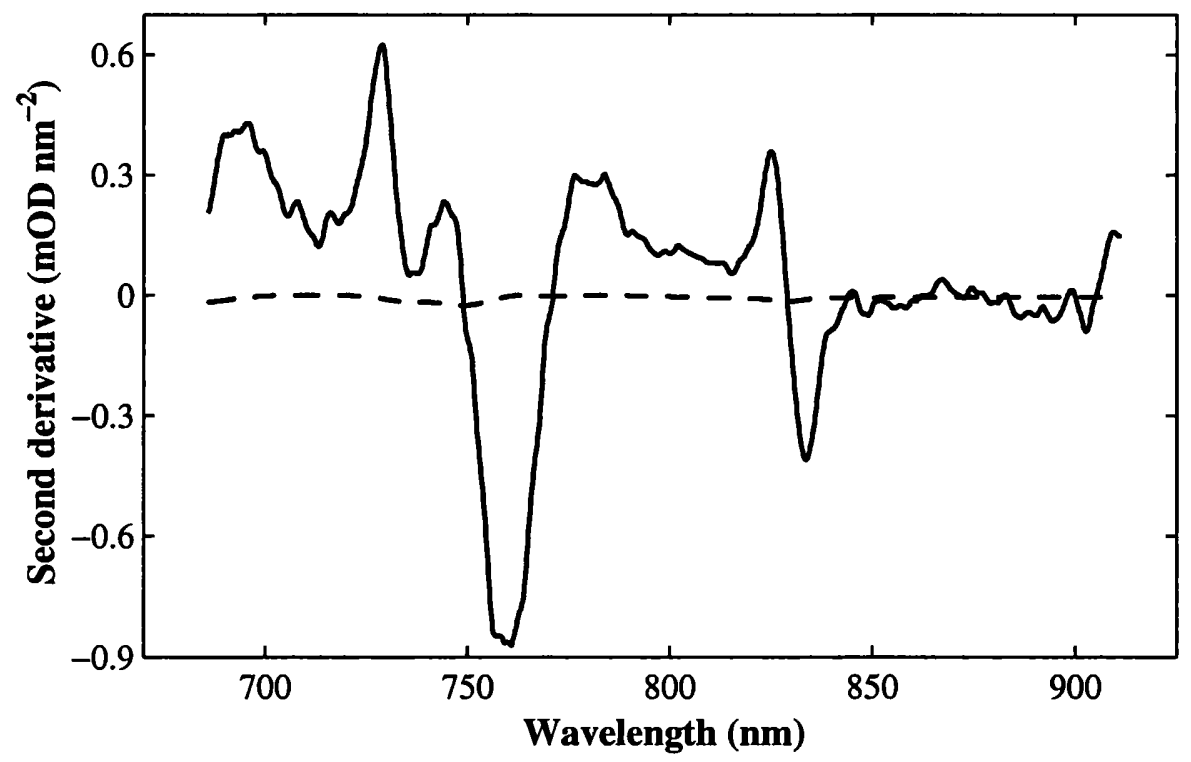

Figure 7.1: The SDS approximation used to determine tissue chromophore concentrations from NIRS measurements of attenuation. The solid line represents the approximated second derivative of a simulated tissue attenuation spectrum, using the first term of Equation (7.1) only. The dashed line represents the sum of the remaining terms.

where the term $\partial A / \partial \mu_{a}$ was defined in Section (3.1) as the differential pathlength (DP). Hence SDS approximates a linear relationship between attenuation and absorption (and thus concentration), the constant of proportionality being the DP. The validity of the SDS approximation relies on the remaining terms in Equation (7.1) being small in comparison to the first one. The scattering coefficient of many tissues can be approximated as having a linear dependence on wavelength in the spectral range of interest (Matcher et al, 1997). In this case, the term containing the second derivative of $\mu_{s}^{\prime}$ will be zero and the other terms containing the first derivative of $\mu_{s}^{\prime}$ will be small, provided $\mu_{s}^{\prime}$ is a relatively weak function of wavelength. Matcher et al (1997) determined the wavelength-dependence of the transport scattering coefficient of various in vivo tissues (measured on the human head, forearm and calf) in the range 760-900 nm, the linear slopes of which were found to be in the range -2.2 to $-3.9 \mathrm{mOD} \mathrm{cm}^{-1}$, i.e. 3 orders of magnitude smaller than $\mu_{s}^{\prime}$ itself. Using typical values for the absorption and transport scattering coefficients of the human forearm in the range 680-920 $\mathrm{nm}$ (Matcher et al, 1994; Matcher et al, 1997), the validity of the approximation in Equation (7.2) was investigated. The partial derivative terms in Equation (7.1) were generated using the analytical solution for reflectance from a semiinfinite medium given in Equation (2.32) (Section 2.3). The derivatives of the $\mu_{a}$ and $\mu_{s}^{\prime}$ spectra were generated numerically using a Savitzsky-Golay filter, the process for which will be described in Section 7.1.2. The second derivative of the simulated attenuation spectrum was then calculated by summing the individual terms in Equation (7.1). Figure 
7.1 shows the second derivative attenuation spectrum and the contribution to it by the sum of all terms other than the first in Equation (7.1). It can be seen that the first term dominates the second derivative and that the remaining terms are generally small in comparison. However, the relative size of the remaining terms and their wavelengthdependence will depend on the exact $\mu_{a}$ and $\mu_{s}^{\prime}$ spectrum underlying the attenuation spectrum.

During practical in vivo studies, therefore, the errors incurred by assuming the SDS approximation will depend on the tissue type under investigation and the wavelength range employed, as documented by Matcher and Cooper (1994). Moreover, as the chromophore concentrations change due to physiological responses, the terms ignored by the approximation will also change, hence the errors will not be constant throughout the monitoring period. Further simulations in Section 7.1.3 will attempt to quantify the errors in concentrations predicted by SDS specifically for measurements of the human forearm.

\subsubsection{The Savitzsky-Golay Filter}

The derivative of a spectrum with respect to wavelength can be calculated using a Savitzsky-Golay filter (Press et al, 1992), which approximates the data within a 'moving window' as a polynomial by means of least-squares fitting, thus smoothing the data. The value for any derivative order can be found, provided the polynomial is of higher order

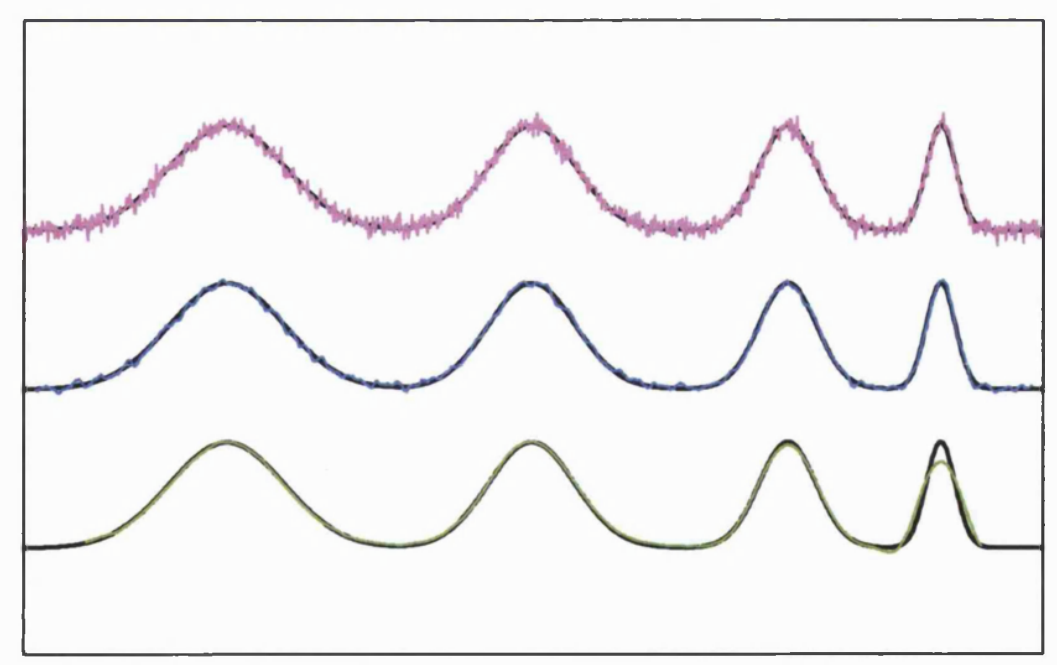

Figure 7.2: Data smoothing by the Savitzsky-Golay filter. The underlying function (-) is a series of Gaussians of varying width, to which normally distributed random noise is added $(-)$, shown in the top plot. The middle plot $(-)$ displays the data smoothed by a 10 -point Savitzsky-Golay filter, the bottom plot (-) is smoothed by a 60 -point filter (see text for more details). 
than that of the required derivative. As the filter width increases the smoothing increases (i.e. random noise is reduced), but the ability to resolve sharp features is degraded.

In previous chapters the Savitzsky-Golay filter has been used to smooth spectral data without differentiating, i.e. filtering in the zeroth order. Figure 7.2 demonstrates the smoothing performance of the filter in the zeroth order. Simulated noisy data is plotted at the top of the figure, the underlying function of which is a series of Gaussians of different widths. The middle and lower plots show the noisy data filtered by a 10-point (half-width) and a 60 -point fourth-order filter respectively. With the 10-point filter the broader bands still appear noisy, but the shape of the narrower bands is preserved. With the 60-point filter the broader bands are smoothed more satisfactorily, but the filter does not accurately resolve the sharper bands. As an approximate guide, the best results for a fourth-order filter are obtained when its full-width is between one and two times the full-width halfmaximum (FWHM) of the narrowest of features in the data (Press et al, 1992). It should be noted that the range of a spectrum once it has been filtered will be shorter by twice the half-width of the filter (i.e. reduced by a half-width at either end of the spectrum).

\subsubsection{Determining Chromophore Concentrations by SDS}

Using the linear relationship in Equation (7.2) between attenuation and absorption in the second derivative, SDS has previously been used to determine the concentrations of tissue

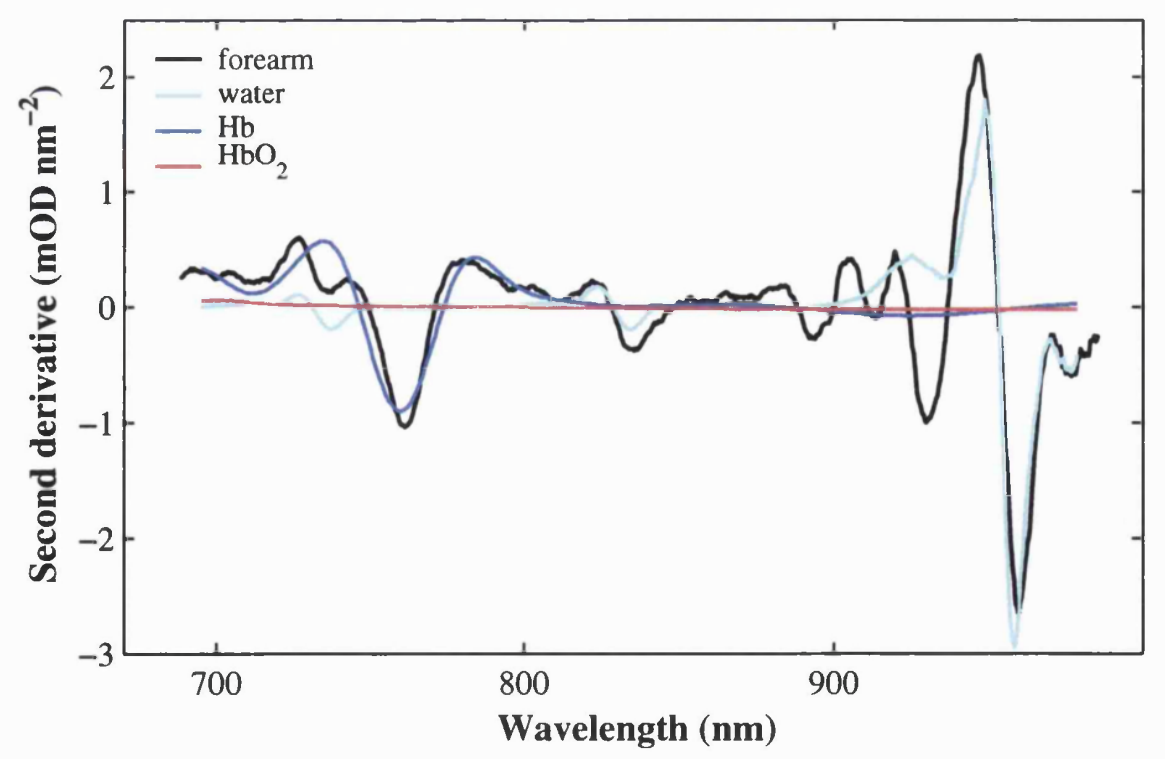

Figure 7.3: Second derivative spectra of human forearm attenuation and tissue chromophore absorption spectra in the range $690-980 \mathrm{~nm}$. The tissue chromophore spectra are scaled to best display their spectral features relative to the tissue spectrum (see text for more details). 
chromophores from in vivo measurements of attenuation (Matcher et al, 1994; Cooper et al, 1996). Figure 7.3 shows the second derivative spectra of an in vivo attenuation spectrum measured on the adult forearm (taken from the results of Section 6.2), and the second derivatives of the absorption spectra of tissue chromophores water, oxy- and deoxyhaemoglobin (scaled arbitrarily to best display their spectral features relative to the tissue spectrum). Comparing the tissue spectrum with the chromophore spectra one can clearly see the contributions to the tissue spectrum from water, at approximately 740,840 and $970 \mathrm{~nm}$, and from $\mathrm{Hb}$ at around $760 \mathrm{~nm}$. In addition, the dip at approximately 930 $\mathrm{nm}$ in the forearm spectrum is due to the lipid absorption band, also visible in the original spectra displayed in Figure 6.4. $\mathrm{HbO}_{2}$ has a very small, featureless second derivative down to about $720 \mathrm{~nm}$, below which it increases gradually.

The process of fitting chromophore spectra to an attenuation spectrum in the second derivative in order to determine chromophore concentrations can be represented using matrices in the same way as the CLS step of the hybrid calibration method in Section 5.3 .4

$$
\begin{aligned}
& \mathbf{A}=\mathbf{R} \cdot \mathbf{K}+\mathbf{E}_{\mathbf{A}} \\
& \hat{\mathbf{R}}=\mathbf{A} \cdot \mathbf{K}^{\prime} \cdot\left(\mathbf{K} \cdot \mathbf{K}^{\prime}\right)^{-1}
\end{aligned}
$$

Here the $(\mathrm{n} \times \mathrm{m})$ data matrix $\mathbf{A}$ contains the second derivative of the attenuation spectra and the $(\mathrm{h} \times \mathrm{m})$ matrix $\mathbf{K}$ contains the second derivatives of the specific absorption spectra of the $\mathrm{h}$ chromophores required. By comparison with the SDS approximation of Equation (7.2), the regression coefficients in the $(\mathrm{n} \times \mathrm{h})$ matrix $\mathbf{R}$ are therefore the chromophore concentrations, multiplied by a pathlength-weighted factor. The true 'non-weighted' concentrations can be recovered if the pathlengths at each wavelength are implicitly included in the fit as follows

$$
\mathbf{A}=\mathbf{C} \cdot(\mathbf{K} \cdot \mathbf{P})+\mathbf{E}_{\mathbf{A}}
$$

where $\mathbf{C}$ contains the true concentrations and $\mathbf{P}$ is an $(\mathrm{m} \times \mathrm{m})$ diagonal matrix, with diagonal elements equal to the $\mathrm{m}$ pathlength values. If the pathlength values in $\mathbf{P}$ are normalised to some wavelength within the fit range, i.e. $\mathbf{P}$ contains the relative wavelengthdependence of the pathlength (WDPL), then a regression of $\mathbf{A}$ on to the WDPL-corrected chromophore spectra, i.e. $(\mathbf{K} \cdot \mathbf{P})$, will yield the true chromophore concentrations multiplied by the absolute pathlength at the normalisation wavelength. Hence, assuming that one chromophore concentration in tissue is accurately known (typically water), the others 
can be determined by multiplying the ratio of the unknown to known chromophore regression coefficients by the known concentration, i.e. dividing by the pathlength. In fact, the initial motivation for the development of the SDS method was the knowledge that water concentration in tissues can be determined to within a few percent (Wray et al, 1988).

For most NIRS measurements of tissue, however, the WDPL is unknown, since it depends on the very parameters the method seeks to find: the chromophore concentrations. If the WDPL is ignored altogether and matrix $\mathbf{A}$ is simply regressed on to the chromophore spectra $\mathbf{K}$, as in Equation (7.3), then the regression coefficients returned will incorporate a $\mathbf{K}$-matrix weighting of the pathlengths, which will vary for the different chromophore concentrations. The effect on the predicted concentrations of ignoring the WDPL, or attempting to correct for it based on prior knowledge, is difficult to describe succinctly, since it will depend on the tissue type and the fitting range used. Using various SDS fitting routines Matcher and Cooper found that they could predict $\mathrm{Hb}$ concentration to within 8 and $11 \%$ from simulations of adult and neonatal brain matter spectra respectively, without correcting for the WDPL. It is generally true that if the change in the WDPL over the fit range is relatively small, the ratios between the second derivative fit coefficients should be approximately equal to the ratio of the true concentrations.

A brief investigation of the ability of the SDS method to predict chromophore concentrations was performed using 'noise-free' simulations of tissue attenuation, generated by the diffusion approximation for reflectance from a semi-infinite medium (Section 2.3). Initially, two tissue attenuation spectra were simulated for the wavelength ranges over which the 740 and $840 \mathrm{~nm}$ water absorption bands were first measured (Method I, described in Section 5.1.2.1), i.e. $650-805 \mathrm{~nm}$ and $765-920 \mathrm{~nm}$. The transport scattering spectra employed in the simulations were calculated from time-resolved measurements of the adult forearm, as mentioned in Section 7.1.1 (Matcher et al, 1997). The total simulated absorption spectra comprised of the water absorption spectra measured at $37^{\circ} \mathrm{C}$ in the respective ranges (Section 5.1.2.1) and the $\mathrm{Hb}$ and $\mathrm{HbO}_{2}$ specific absorption spectra displayed in Figure 2.2, combined at concentrations relevant to the adult forearm (Matcher et al, 1994; Matcher et al, 1997). The water concentration was assumed to be $60 \%$ and the total haemoglobin concentration was $100 \mu \mathrm{M}$, with a mean tissue oxygen saturation $\left(\mathrm{S}_{\mathrm{mt}} \mathrm{O}_{2}\right)$ of $65 \%$, i.e. $65 \mu \mathrm{M}$ of $\mathrm{HbO}_{2}$ and $35 \mu \mathrm{M}$ of $\mathrm{Hb}$. The pathlengths were calculated from the expression for the DP given in Equation (3.7), the partial differential of attenuation with respect to $\mu_{a}$, and were normalised to unity at 740 and $840 \mathrm{~nm}$ for the two ranges respectively. Table 7.1 shows the results of fitting the chromophore spectra 


\begin{tabular}{llllll}
\hline $\begin{array}{l}\text { Fit range } \\
(\mathrm{nm})\end{array}$ & $\begin{array}{l}\text { Chromophores } \\
\text { included in fit }\end{array}$ & $\begin{array}{l}{[\mathrm{Hb}]} \\
(\mu \mathrm{M})\end{array}$ & \% error & $\begin{array}{l}{\left[\mathrm{HbO}_{2}\right]} \\
(\mu \mathrm{M})\end{array}$ & \% error \\
\hline $650-805$ & All & 32.5 & 7.1 & 48.0 & 26.1 \\
& $\mathrm{Hb}+\mathrm{H}_{2} \mathrm{O}$ & 31.6 & 9.6 & - & - \\
$765-920$ & All & 34.5 & 1.4 & 80.3 & 23.5 \\
& $\mathrm{Hb}+\mathrm{H}_{2} \mathrm{O}$ & 36.3 & 3.8 & - & - \\
$720-880$ & $\mathrm{All}$ & 34.0 & 3.0 & 61.0 & 6.2 \\
& $\mathrm{Hb}+\mathrm{H}_{2} \mathrm{O}$ & 34.5 & 1.3 & - & - \\
\hline
\end{tabular}

Table 7.1: SDS-estimated chromophore concentrations using simulated forearm attenuation spectra and WDPL-corrected chromophore absorption spectra, (see text for details).

to the simulated attenuation spectra in the second derivative, including the correction for the WDPL as described by Equation (7.4). The errors in the estimated concentrations for the first range $(650-805 \mathrm{~nm})$ were relatively high, especially for $\mathrm{HbO}_{2}$ in the fit including all three chromophores. The second derivative spectrum of $\mathrm{HbO}_{2}$ is relatively small and flat, as displayed in Figure 7.3, which would explain the larger errors for this chromophore. However, the spectrum does have some structure, therefore excluding it from the fit altogether degrades the estimation of the $\mathrm{Hb}$ concentration. The errors on the $\mathrm{Hb}$ concentration in the fit range $765-920 \mathrm{~nm}$ were smaller than for the range $650-805$ $\mathrm{nm}$, though the $\mathrm{HbO}_{2}$ concentration error was approximately the same. Previous studies have shown that fitting to a range that includes both the 740 and $840 \mathrm{~nm}$ water bands improves the accuracy of concentration estimation by SDS due to the increased information content in the $\mathbf{K}$ matrix (Matcher and Cooper, 1994), which reduces errors caused by co-linearities (or 'cross-talk') between the chromophore spectra (see Section 5.3). The two measured water ranges were therefore temperature-matched together (as described in Section 5.1.2.3), and the SDS analysis re-applied to the new attenuation spectrum, generated using the same chromophore concentrations as for the first two ranges. The upper limit to the range of the fit was set to $880 \mathrm{~nm}$, since beyond this wavelength there is very little additional information to be gained from the second derivative spectra (see Figure 7.3). The optimal fit range was then found to be $720-880 \mathrm{~nm}$, the estimated concentrations for which are also given in Table 7.1. The estimated $\mathrm{HbO}_{2}$ concentration for the fit of all three chromophores to the extended range is a vast improvement on both the previous ranges. Excluding $\mathrm{HbO}_{2}$ from the fit again improves the $\mathrm{Hb}$ concentration estimation, most likely due to the reduction in cross-talk between the chromophore spectra.

In order to determine the effect of the WDPL correction on the results, the fit to the 


\begin{tabular}{llllll}
\hline Correction & $\begin{array}{l}\text { Chromophores } \\
\text { included in fit }\end{array}$ & $\begin{array}{l}{[\mathrm{Hb}]} \\
(\mu \mathrm{M})\end{array}$ & \% error & $\begin{array}{l}{\left[\mathrm{HbO}_{2}\right]} \\
(\mu \mathrm{M})\end{array}$ & \% error \\
\hline I & All & 34.0 & 3.0 & 61.0 & 6.2 \\
& $\mathrm{Hb}+\mathrm{H}_{2} \mathrm{O}$ & 34.5 & 1.3 & - & - \\
II & $\mathrm{All}$ & 34.1 & 2.5 & 84.8 & 30.4 \\
& $\mathrm{Hb}+\mathrm{H}_{2} \mathrm{O}$ & 34.9 & 0.2 & - & - \\
$\mathrm{III}$ & $\mathrm{All}$ & 33.1 & 5.6 & 59.7 & 8.2 \\
& $\mathrm{Hb}+\mathrm{H}_{2} \mathrm{O}$ & 33.6 & 4.0 & - & - \\
\hline
\end{tabular}

Table 7.2: The effect of different WDPL corrections of the chromophore spectra on the SDS-estimated chromophore concentrations of a simulated forearm attenuation spectrum. Correction I is the original (true) WDPL, correction II is no WDPL and correction III is as for I but with a $\mathrm{S}_{\mathrm{mt}} \mathrm{O}_{2}$ of $75 \%$ instead of $65 \%$.

720-880 nm attenuation spectrum was repeated using two additional WDPL corrections. The results for the estimated chromophore concentrations for the extended range, using the three WDPL corrections listed below, are shown in Table 7.2:

I: the original (correct) WDPL

II: no WDPL, i.e. fitting with the pure (second derivative) chromophore spectra

III: a WDPL in which the $\mathrm{S}_{\mathrm{mt}} \mathrm{O}_{2}$ was altered from $65 \%$ (in the original) to $75 \%$, another in the range of $\mathrm{S}_{\mathrm{mt}} \mathrm{O}_{2}$ values reported in the literature (Doornbos et al, 1999).

Interestingly the results in Table 7.2 for correction II indicate that the error in the $\mathrm{Hb}$ concentration estimations are actually smaller when the WDPL is ignored, although the $\mathrm{HbO}_{2}$ error is significantly larger. For correction III, in which the $\mathrm{S}_{\mathrm{mt}} \mathrm{O}_{2}$ was increased, the errors are slightly larger than those found for the original correction. It has previously been shown (Kohl et al, 1998) that the WDPL is most strongly affected by the $\mathrm{S}_{\mathrm{mt}} \mathrm{O}_{2}$, as opposed to the absolute chromophore concentrations employed. Figure 7.4 shows the three WDPL corrections in the range $720-880 \mathrm{~nm}$, normalised at $800 \mathrm{~nm}$. Essentially, as the $\mathrm{S}_{\mathrm{mt}} \mathrm{O}_{2}$ changes the slope of the WDPL changes, which affects the errors in the estimated concentrations in different ways. The results in Table 7.2 suggest that in this regime, i.e. this particular choice of absorption and scattering spectra, the effect of the WDPL correction, generated using a $\mathrm{S}_{\mathrm{mt}} \mathrm{O}_{2}$ within the physiological range, on the estimated concentrations is relatively small. In practical tissue measurements, therefore, an estimation of the WDPL could be made based on typical values from the literature, e.g. (Essenpreis et al, 1993), whilst noting that its slope will affect the SDS analysis. Moreover, if a fixed 


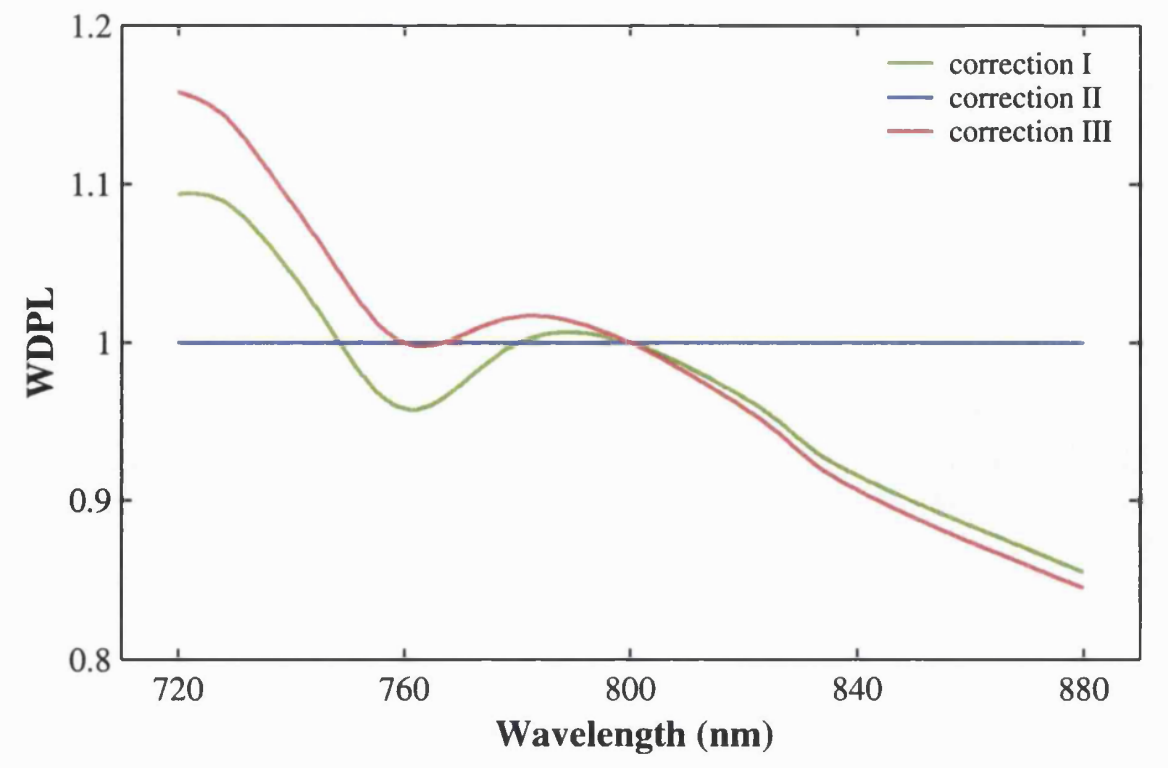

Figure 7.4: WDPL corrections in the range 720-880 $\mathrm{nm}$ used in the estimation of chromophore concentrations by SDS, see text for details.

WDPL correction is applied to the entire data (as is generally the case), any changes in the underlying absorption and scattering spectra of the tissue will cause additional shifts in the errors of the SDS-predicted concentrations.

\subsubsection{Temperature Prediction}

In Section 5.3.5 water temperature was calibrated against the concentrations of loading vectors determined from a PCA of the water absorption spectrum, measured as a function of temperature. As described in Section 7.1.3, SDS applied to tissue measurements involves the direct determination of component concentrations by approximating a linear relationship between attenuation and absorption. Therefore it should, in theory, be possible to predict tissue temperature by the following two-stage process:

i) fitting to the second derivative of the tissue attenuation spectra with the second derivative water loading vectors and chromophore specific absorption spectra using Equation (7.4)

ii) using the concentrations (or scores) of the water loading vectors found in step i) to obtain temperature by multiplying them with the calibration vector $\mathbf{v}$ determined from the PCR step in Equation (5.44).

There are several factors, however, that may limit the accuracy of temperature prediction by this method. Firstly, the scores of the water loading vectors in the mean-centered 
water absorption data set are small in comparison to the mean absorption spectrum, which will therefore bias the least-squares fit against their accurate determination (relative to the concentration of the mean spectrum). Noise on the water loading vectors will be amplified in the second derivative, due to the numerical process of the filter (Press et al, 1992), and may degrade the accuracy to which the scores can be determined. Furthermore, the smoothing of the data performed by the filter may alter the underlying structure of the water loading vectors significantly, such that their true scores will not be found. A preliminary study was performed, therefore, to establish whether the temperature of water alone could be predicted using the proposed SDS method from the measurements of the water absorption spectrum as a function of temperature.

Since the optimal fit range for a typical tissue attenuation spectrum was determined in Section 7.1.3 to be $720-880 \mathrm{~nm}$, the PCR calibration of the water absorption spectrum (measured by Method I, see Section 5.1.2.1) was repeated for the temperature-matched ranges, including both the 740 and $840 \mathrm{~nm}$ water bands. The standard errors of calibration and prediction, the latter determined by the cross-validation method described in Section 5.3.5, and their correlation coefficients for the new PCR calibration are given in Table 7.3. The second derivatives of the water absorption spectra were fitted with the second derivatives of the water loading vectors in the range $720-880 \mathrm{~nm}$, ensuring that this was the filtered fit range, since the process of filtering reduces the spectral range prior to fitting (see Section 7.1.2). A suitable width of filter with which to differentiate the data was estimated by fitting a single Gaussian function to the first loading vector and determining its FWHM. As stated in Section 7.1.2, the full width of the optimal filter should be 1-2 times the FWHM of the underlying 'functions', thus the filter width was set to $15 \mathrm{~nm}$. Since the water absorption spectra were mean-centered for the PCR calibration in the zero order, the fit to the second derivative water spectra must also include the second derivative of the mean water absorption spectrum from the calibration. Although the mean calibration spectrum could be subtracted from (rather than fitted to) the data, in

\begin{tabular}{lllll}
\hline PCs & SEC $\left({ }^{\circ} \mathrm{C}\right)$ & $r_{c}^{2}$ & SEP $\left({ }^{\circ} \mathrm{C}\right)$ & $r_{p}^{2}$ \\
\hline 1 & 0.8082 & 0.9579 & 0.8138 & 0.9573 \\
2 & 0.1117 & 0.9992 & 0.1131 & 0.9992 \\
3 & 0.0877 & 0.9995 & 0.0892 & 0.9995 \\
4 & 0.0650 & 0.9997 & 0.0668 & 0.9997 \\
\hline
\end{tabular}

Table 7.3: Statistical parameters for the PCR calibration of the temperature-matched ranges of the water absorption spectrum measured by Method I. 


\begin{tabular}{lll}
\hline PCs & SEP $\left({ }^{\circ} \mathrm{C}\right)$ & $r_{p}^{2}$ \\
\hline 1 & 0.3800 & 0.9907 \\
2 & 0.3658 & 0.9914 \\
3 & 0.3894 & 0.9903 \\
4 & 0.3845 & 0.9906 \\
\hline
\end{tabular}

Table 7.4: Standard error for water temperature prediction using the SDS method, applied to the measured water absorption spectra, including up to four PCs, see text for details.

practical tissue measurements its concentration, i.e. the actual water concentration, is not $100 \%$ as it is here, therefore the mean spectrum is included in the fit in order to distinguish its contribution from those of the loading vectors.

Water temperature was then determined by multiplying the concentrations or 'scores' of the loading vectors in the second derivative by the calibration vector $\mathbf{v}$, as described in Section 5.3.5. Table 7.4 shows the SEPs and correlation coefficients, for the inclusion of up to four PCs, for temperature prediction by this method. In this case the SEP is calculated from the temperature predictions of the method, not by the cross-validation method used for the calibration. It can be seen that the temperature prediction is not improved beyond the inclusion of two PCs. A study of the loading vectors in the second derivative (not shown) reveals that beyond two PCs the loading vectors have no significant features, therefore will contribute only noise to the predicted temperature value. The correlation between the actual (measured) and predicted temperature using the SDS method is still reasonable at $\sim 0.4^{\circ} \mathrm{C}$, although noise amplification in the second derivative has increased the SEPs compared to those for the calibration in the zero order (Table 7.3). However, the range over which the PCR calibration was performed was not the same as that for the second derivative fit, which may affect the temperature prediction. Since the process of differentiating the data reduces its spectral range, it is not possible to fit in the second derivative over the exact same range for which the calibration was performed.

The next step was to apply the SDS temperature prediction method to a simulated attenuation data set in which the water absorption spectrum varied with temperature. The simulated attenuation spectra were generated in the manner described in Sections 7.1.1 and 7.1.3 for the adult forearm spectrum, using the water absorption spectra measured as a function of temperature between 44.2 and $32.5^{\circ} \mathrm{C}$. The chromophore concentrations were fixed such that the only changes in the data were due to the temperature-dependence of the water absorption spectrum (in real tissue this would not however be the case). The mean calibration spectrum and the first two loading vectors from the water calibration 


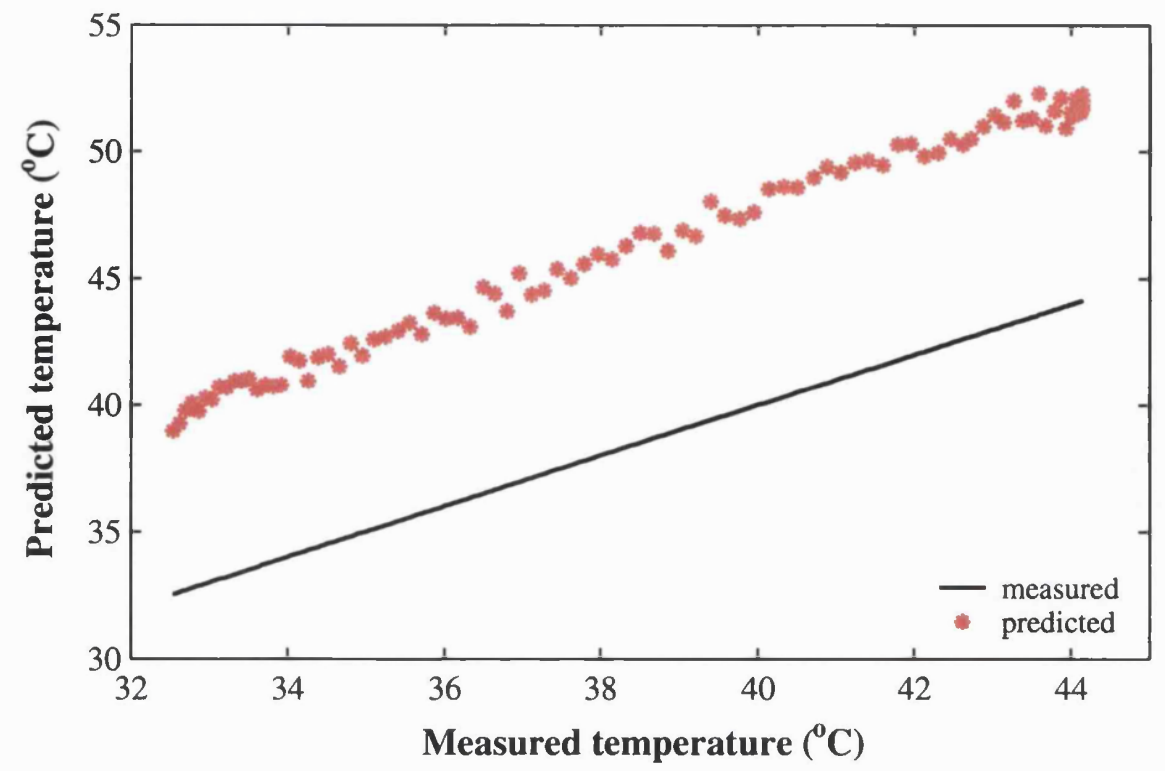

Figure 7.5: Predicted against measured temperature using the SDS method on a simulated tissue attenuation data set, see text for details.

were included in the second derivative fit, as were the $\mathrm{Hb}$ and $\mathrm{HbO}_{2}$ spectra. The scores for the water loading vectors were recovered by dividing the regression coefficients for the loading vectors by those for the mean calibration spectrum, i.e. correcting the scores for the percentage of water used in the simulation. As can be seen in Figure 7.5, the predicted temperature greatly over-estimates the measured temperature by $\sim 7-8^{\circ} \mathrm{C}$, an error which increased when excluding $\mathrm{HbO}_{2}$ from the fit. Moreover, the error is linearly dependent on the actual measured temperature. It is probable that there is a significant amount of cross-talk between the fit components, which would explain the poor prediction of temperature based on the concentration values (i.e. the scores) determined by the fit.

Although SDS may provide reasonable estimates of the chromophore concentrations during a 'steady-state', assuming an optimal range is found for the regime under study, the prediction of temperature based on small changes in the loading vectors results in large errors due to noise inflation and cross-talk between the components in the second derivative. Due to the poor results obtained using the simulated data, therefore, the application of the SDS technique to the temperature prediction of the in vivo data will not be reported. However, the method was applied to the attenuation measurements of the adult forearm to determine the chromophore concentrations during the initial 'steadystate' prior to cooling, as will be described in Section 7.2.2. 


\subsection{Algorithm II: Non-Linear Optimisation Method}

Algorithm II involves a non-linear optimisation technique based on a diffusion theory approximation for attenuation through a semi-infinite medium. The technique was employed to reconstruct absorption from measurements of attenuation as a function of temperature. On the basis that the absorption spectra can be accurately recovered, one can then attempt to predict temperature by applying the calibration of the water spectrum described in Section 5.3. Algorithm II was applied to the attenuation spectra measured on the adult forearm (details in Section 6.2) during cooling. This section will firstly describe the theory behind the non-linear optimisation technique and the assumptions it makes. The technique is then applied to the in vivo forearm data, using the PCR calibration of the water spectrum (Section 5.3.5) to predict tissue temperature in the forearm from the reconstructed absorption spectra.

\subsubsection{Theory}

The non-linear optimisation technique compares the measured attenuation spectra to a diffusion theory based model of attenuation and attempts to find the optimal absorption coefficient such that the difference between modelled and experimentally determined attenuation is minimised. In actual fact, the technique will attempt to find the changes in absorption from some baseline point, as opposed to the absolute absorption. This is because, as discussed in Section 3.1, the lack of photometric calibration in the measurement system makes a truly absolute measurement of attenuation difficult to obtain. Hence the algorithm will be applied to the attenuation difference spectra, i.e. the changes in attenuation from the chosen baseline (see Section 3.1). Assuming, in the case of the adult forearm data, that the tissue is at a stable and uniform temperature prior to cooling, (ensured by the zero-heat flow method, see Section 6.2), this baseline is taken to be the initial measurement at a temperature of $37^{\circ} \mathrm{C}$. The measured difference attenuation is then given by

$$
\triangle A_{\text {meas }}=A-A_{\text {cal }}
$$

where $A_{c a l}$ is the attenuation determined from the initial measurement, used as the calibration spectrum, and $A$ is any subsequent measurement of attenuation. Because of the non-linear relationship between attenuation and absorption (Section 3.1), the change in attenuation cannot be described as being a function of the change in absorption, i.e. $A\left(\mu_{a}\right)-A\left(\mu_{a c a l}\right) \neq \triangle A\left(\Delta \mu_{a}\right)$ where $\Delta \mu_{a}=\mu_{a}-\mu_{a c a l}$. This means that in order to de- 
termine the change in absorption $\Delta \mu_{a}$ one must know the underlying absorption $\mu_{a \text { cal }}$ of the calibration spectrum. Moreover, attenuation is a function of both absorption and scattering, so the transport scattering coefficient of the calibration spectrum must also be known. Hence, in using this technique it is assumed that the optical properties $\mu_{a}$ and $\mu_{s}^{\prime}$ of the tissue in its initial state are known. Since the optimisation technique is attempting to determine one unknown, $\Delta \mu_{a}$, from one measurement of (difference) attenuation, an additional assumption is that $\mu_{s}^{\prime}$ does not change during the experiment. Both these points will be discussed in more detail in Section 7.2.4.

To determine changes in absorption with temperature, the measured difference attenuation spectra, calculated using Equation (7.5), were compared to a solution of the diffusion approximation for the steady-state spatially-resolved reflectance from a semi-infinite turbid medium (see Section 2.3). The solution employed here, given in Equation (2.32), was derived using the zero boundary condition (Patterson et al, 1989). It follows from Equation (2.32) that the attenuation measured at a distance $\rho$ from the incident pencil beam is given by

$$
\begin{aligned}
A(\rho) & =-\log _{10} R(\rho) \\
& =\log _{10}\left(2 \pi \mu_{t}^{\prime} r^{2}\right)-\log _{10}\left(\mu_{e f f}+\frac{1}{r}\right)+\frac{\mu_{e f f} r}{2.3}
\end{aligned}
$$

where $r$ is the distance from the observation point $\rho$ to the isotropic point source at $1 / \mu_{t}^{\prime}$ below the surface of the medium and $\mu_{\text {eff }}$ is the effective attenuation coefficient (see Section 2.3). The modelled difference attenuation, with respect to that for a set of known or calibration optical properties, is then given by

$$
\triangle A_{m o d}=\log _{10}\left(\frac{\mu_{t}^{\prime}}{\mu_{t c a l}^{\prime}}\right)+\log _{10}\left(\frac{\mu_{e f f c a l}+1 / r}{\mu_{e f f}+1 / r}\right)+\frac{\left(\mu_{e f f}-\mu_{e f f c a l}\right)}{2.3} r
$$

where $\mu_{\text {eff cal }}$ and $\mu_{t c a l}^{\prime}$ describe the optical properties of the calibration spectrum.

The optimisation technique attempts to reconstruct the difference absorption coefficient $\Delta \mu_{a}$ by finding the value of $\Delta \mu_{a}$ for which the following function approaches zero

$$
\mathrm{F}=\triangle A_{\text {mod }}-\triangle A_{\text {meas }}
$$

Based on an initial (user-defined) estimate of $\triangle \mu_{a}$ the technique calculates $\triangle A_{\text {mod }}$ and determines F using Equation (7.8). Depending on whether F is positive or negative the estimate for $\triangle \mu_{a}$ is increased or decreased and $\mathrm{F}$ is recalculated. This process is iteratively 
repeated until the function $F$ is, to within some threshold value, equal to zero. At the point of convergence, when $\mathrm{F}$ is equal or close zero, we have $\triangle A_{\text {mod }} \approx \Delta A_{\text {meas. }}$. Assuming the model is a good approximation to the measurement, therefore, the change in the absorption of the medium is inferred by the final-iteration estimate of $\Delta \mu_{a}$.

For Algorithm II, the iterative process described above was performed using the Newton-Raphson root-finding method, also known as Newton's method (Press et al, 1992; Gill et al, 1981). In this process the non-linear function $\mathrm{F}(x)$ of which one wants to find the root, i.e. the value of $x$ for which the function itself is zero, is approximated by a linear function at each iteration. The Newton-Raphson method is based on the Taylor series expansion of a function in the neighbourhood of a point $x$

$$
\mathrm{F}(x+\delta) \approx \mathrm{F}(x)+\delta \mathrm{F}^{\prime}(x)+\delta^{2} \frac{\mathrm{F}^{\prime \prime}(x)}{2}+\cdots \quad \text { (higher order terms) }
$$

where $\mathrm{F}^{\prime}$ and $\mathrm{F}^{\prime \prime}$ are the first and second derivatives of $\mathrm{F}$ respectively. If $\delta$ is small then Equation (7.9) can be approximated by the first two terms only

$$
\mathrm{F}(x+\delta) \approx \mathrm{F}(x)+\delta \mathrm{F}^{\prime}(x)
$$

In the Newton-Raphson method an initial value of $x, x_{1}$, believed to be close to the real root is chosen. As depicted in Figure 7.6, the tangent line (local derivative) at $x=x_{1}$ is extended to $\mathrm{F}(x)=0$ to find a closer estimate of the root $x_{2}$, i.e. the function $\mathrm{F}$ is

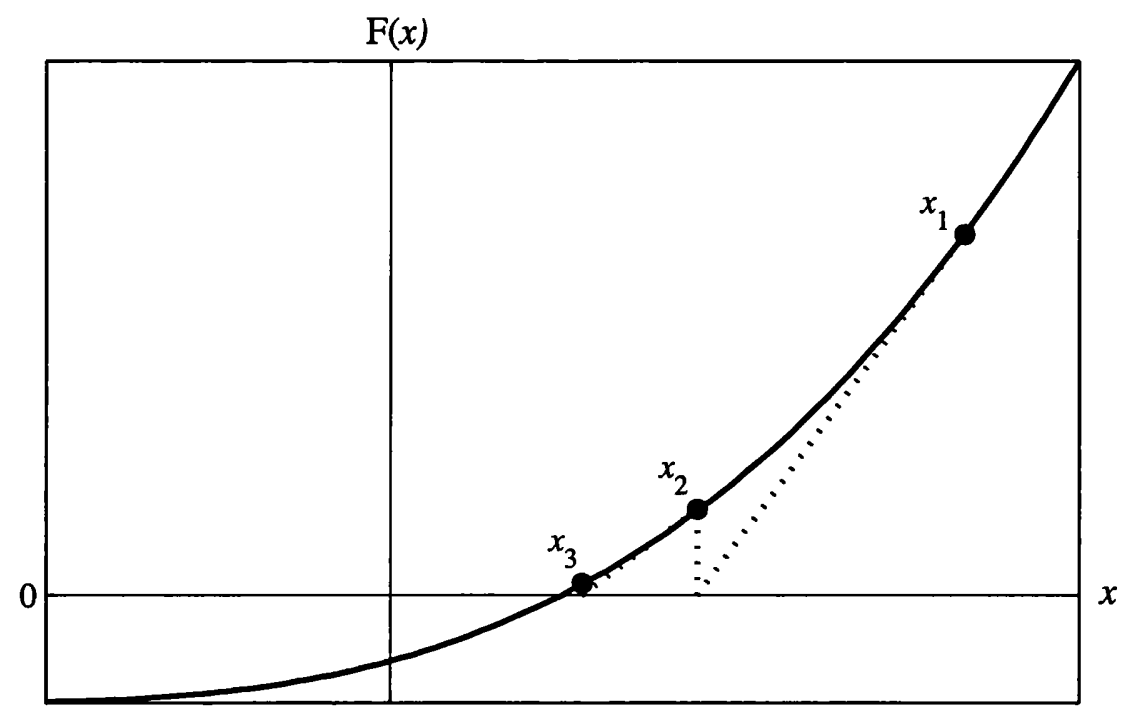

Figure 7.6: Graphical demonstration of the Newton-Raphson root-finding method. An initial estimate $x_{1}$ of the root of $\mathrm{F}(x)$ is made and the local derivative is extrapolated to find the next estimate $x_{2}$ (see text for details). 
approximated by the linear relationship in Equation (7.10). The next estimate of $x, x_{2}$, is determined by setting $\mathrm{F}\left(x_{2}\right)$ to zero in Equation (7.10) as follows

$$
\begin{aligned}
\mathrm{F}\left(x_{2}\right) & =\mathrm{F}\left(x_{1}+\delta\right)=0 \\
x_{2} & =x_{1}+\delta \\
& \approx x_{1}-\frac{\mathrm{F}\left(x_{1}\right)}{\mathrm{F}^{\prime}\left(x_{1}\right)}
\end{aligned}
$$

In the general case the process iteratively updates the estimate of the root as follows

$$
x_{i+1}=x_{i}-\frac{\mathrm{F}\left(x_{i}\right)}{\mathrm{F}^{\prime}\left(x_{i}\right)}
$$

until such time as a (user-defined) 'threshold' value of either the function $\mathrm{F}$ or the change in $x, \delta=x_{i+1}-x_{i}$, is reached, upon which the root is inferred by the current value $x_{i+1}$. In this case, the optimisation process (implemented by a program written in MATLAB) continued until the value of $\mathrm{F}$ defined in Equation (7.8) was less than $0.01 \mathrm{mOD}$, or the value of $\delta$ was less than $0.001 \mathrm{mOD} \mathrm{cm}^{-1}$.

To investigate the ability of the optimisation technique to recover the change in the absorption coefficient from measurements of difference attenuation, the method was applied to Monte Carlo simulations of diffuse reflectance (see Section 2.3). The Monte Carlo simulations, supplied by a co-worker (Okada, 2002), are based on the model for a semiinfinite homogeneous medium described elsewhere (van der Zee and Delpy, 1987). In these simulations ten million photons were 'injected' into the medium and detected $3 \mathrm{~cm}$ away on the surface, assuming fibre diameters of $5 \mathrm{~mm}$ and a refractive index match at the boundary. Four sets of optical coefficients, given in Table 7.5, were used to simulate values of the diffuse reflectance, and attenuation was calculated from the log (base 10) of the reflectance values. Two sets of difference attenuation values were then generated: the difference in attenuation calculated from Simulations I and II, i.e. $\triangle A_{1}=A_{\mathrm{I}}-A_{\mathrm{II}}$, in

\begin{tabular}{lll}
\hline Simulation & $\begin{array}{l}\mu_{a} \\
\left(\mathrm{OD} \mathrm{cm}^{-1}\right)\end{array}$ & $\begin{array}{l}\mu_{s}^{\prime} \\
\left(\mathrm{OD} \mathrm{cm}^{-1}\right)\end{array}$ \\
\hline I & 0.043 & 4.34 \\
II & 0.087 & 4.34 \\
III & 0.043 & 6.51 \\
IV & 0.087 & 6.51 \\
\hline
\end{tabular}

Table 7.5: Optical coefficients used in Monte Carlo simulations of diffuse reflectance from a semi-infinite medium, with a source-detector separation of $3 \mathrm{~cm}$. 
which the value of $\mu_{a c a l}$ (the known $\mu_{a}$ of the 'calibration' measurement) is given by the $\mu_{a}$ of Simulation I, and the difference in attenuation calculated from Simulations III and IV, i.e. $\triangle A_{2}=A_{\mathrm{IV}}-A_{\mathrm{III}}$, in which $\mu_{a \text { cal }}$ is given by the $\mu_{a}$ value of Simulation II. In both cases $\mu_{a}$ changed and $\mu_{s}^{\prime}$ remained the same, as is the assumption in the application of this technique to the tissue attenuation measurements. The optimisation program was initially tested using the correct values of $\mu_{s}^{\prime}$ and $\mu_{a \text { cal }}$, and the correct value for the estimate of $\triangle \mu_{a}$, i.e. $0.043 \mathrm{OD} \mathrm{cm}^{-1}$ for both $\triangle A_{1}$ and $\triangle A_{2}$. At convergence (in less than 25 iterations), the $\triangle \mu_{a}$ values were determined to within 2.3 and $3.2 \%$ of the actual values for $\triangle A_{1}$ and $\triangle A_{2}$ respectively. On altering the initial estimate of $\triangle \mu_{a}$ between 0.1 and 10 times its true value, convergence to the same percentage error was achieved within 50 iterations. The effects on the determination of $\Delta \mu_{a}$ of altering the input values of $\mu_{a c a l}$ and $\mu_{s}^{\prime}$, (i.e. the user-defined estimates of these), in the range of 0.1-10 times their actual values are shown in Figure 7.7. The input values of $\mu_{a \text { cal }}$ are varied from $0.0043-0.2170$ OD cm${ }^{-1}$, i.e. 0.1 to 5 times the actual $\mu_{a c a l}$ value, and the $\mu_{s}^{\prime}$ values are varied from 2.17-43.40 $\mathrm{OD} \mathrm{cm}^{-1}$ i.e. 0.5 to 10 times greater than the actual $\mu_{s}^{\prime}$. In all cases the actual value of $\Delta \mu_{a}$ was input as the initial estimate. As shown in the left-hand graph of Figure 7.7, the true $\triangle \mu_{a}$ is increasingly overestimated when the ratio of input to actual $\mu_{a \text { cal }}$ increases above 1 , and increasingly underestimated as the ratio decreases below 1 . The percentage errors in $\triangle \mu_{a}$ are greater for the difference attenuation with the higher $\mu_{s}^{\prime}$ value
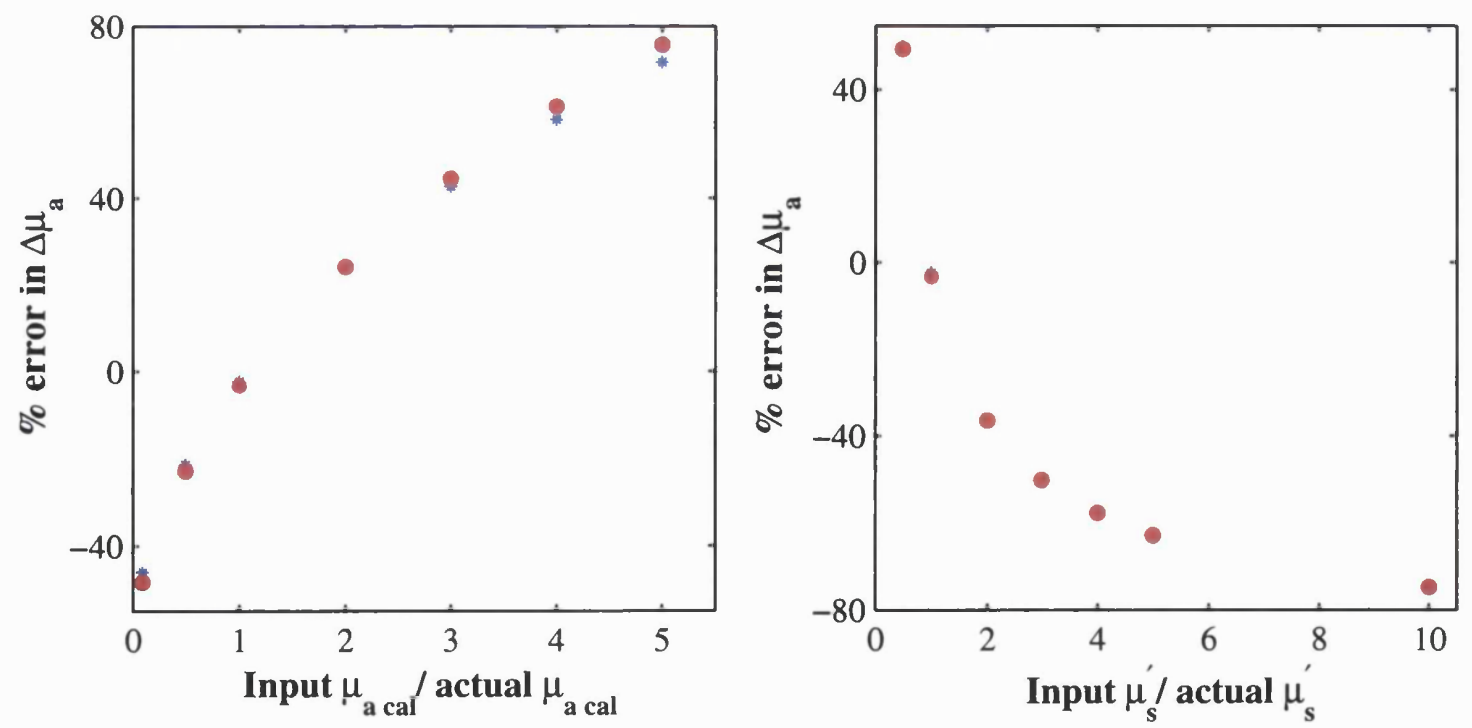

Figure 7.7: Percentage errors in the difference absorption determined by the NewtonRaphson optimisation routine as a function of the ratio of input to actual calibration $\mu_{a}$ and $\mu_{s}^{\prime}$ values, for $\Delta A_{1}\left(^{*}\right)$ and $\Delta A_{2}(\bullet)$. The input $\mu_{a c a l}$ values are varied between 0.1 and 5 times the actual $\mu_{a c a l}$, and the input $\mu_{s}^{\prime}$ values are varied between 0.5 and 10 times the actual $\mu_{s}^{\prime}$. 
$\left(\triangle A_{2}\right)$. In the right-hand graph it can be seen that $\Delta \mu_{a}$ is increasingly underestimated as the ratio of input to actual $\mu_{s}^{\prime}$ increases, and is increasingly overestimated as the ratio decreases below 1 . The difference in the percentage errors between $\triangle A_{1}$ and $\triangle A_{2}$ is less significant for $\mu_{s}^{\prime}$ than it is for $\mu_{a c a l}$. Figure 7.7 therefore illustrates the importance of correctly assuming the underlying $\mu_{a}$ and $\mu_{s}^{\prime}$ spectra of the calibration measurement to which the difference attenuation is referenced.

The following section will apply the non-linear optimisation technique to the reconstruction of tissue absorption spectra using the attenuation data measured on the adult forearm as a function of temperature.

\subsubsection{Application to in Vivo Tissue Data}

As discussed in the previous section, in order to accurately recover the underlying absorption spectrum from measurements of attenuation using the Newton-Raphson method, the absorption spectrum of a reference or calibration measurement must be known (in addition to knowledge of the transport scattering spectrum). For the in vivo forearm data, therefore, the absorption of the 'calibration' spectrum, taken to be the first measurement at $37^{\circ} \mathrm{C}$, was estimated using the SDS method detailed in Section 7.1.

In fact, the SDS method was applied to the first 50 tissue attenuation spectra, as opposed to the first single measurement, to obtain a better estimate of the mean concentrations. During the first 50 measurements the temperature varied by less than $0.1^{\circ} \mathrm{C}$, hence it was assumed that the data set would represent a physiological 'steady-state' in which the chromophore concentrations vary minimally. As for the SDS simulations of Section 7.1.3, the water absorption spectrum at $37^{\circ} \mathrm{C}$ was used in the fit to the second derivative attenuation spectra. Moreover, a fit range of $720-880 \mathrm{~nm}$, found to be optimal for the SDS simulations, was again used. Since the errors in the SDS estimation of $\mathrm{Hb}$ concentration for the simulated data varied by less than $4 \%$ for a wide range of WDPL corrections, (see Table 7.2), the original correction (Correction I) used for the simulated forearm data was applied in the analysis of the in vivo measurements. The regression coefficients, i.e. (concentration $\times$ pathlength), determined from fits including and excluding $\mathrm{HbO}_{2}$ are given in Table 7.6. As expected from previous simulations (Section 7.1.3), the $\mathrm{HbO}_{2}$ regression points are extremely noisy, with a coefficient of variation (CV) of $\sim 50 \%$. Therefore, only the fits with $\mathrm{Hb}$ and water were considered, which altered the regression coefficients for both $\mathrm{Hb}$ and water by less than $1 \%$, within the standard deviation of those obtained from the fit including $\mathrm{HbO}_{2}$. As a brief investigation of the effect of the WDPL 


\begin{tabular}{lllllll}
\hline $\begin{array}{l}\text { Chromophores } \\
\text { included in fit }\end{array}$ & $\begin{array}{l}{[\% \cdot \mathrm{H} /} \\
(\%)\end{array}$ & $\begin{array}{l}\mathrm{CV} \\
(\%)\end{array}$ & $\begin{array}{l}{[\mathrm{Hb}] \times \mathrm{DP}} \\
(\mu \mathrm{M} \cdot \mathrm{cm})\end{array}$ & $\begin{array}{l}\mathrm{CV} \\
(\%)\end{array}$ & $\begin{array}{l}{\left[\mathrm{HbO}_{2}\right] \times \mathrm{DP}} \\
(\mu \mathrm{M} \cdot \mathrm{cm})\end{array}$ & $\begin{array}{l}\mathrm{CV} \\
(\%)\end{array}$ \\
\hline All & $5.42 \pm 0.23$ & 4.2 & $210.80 \pm 7.89$ & 3.7 & $280.18 \pm 141.02$ & 50.3 \\
$\mathrm{Hb}+\mathrm{H}_{2} \mathrm{O}$ & $5.37 \pm 0.22$ & 4.17 & $211.10 \pm 7.88$ & 3.7 & - & - \\
\hline
\end{tabular}

Table 7.6: SDS-estimated chromophore concentrations of the first 50 attenuation spectra measured on the adult forearm, including and excluding $\mathrm{HbO}_{2}$ in the fit.

on the fit coefficients, the $\mathrm{S}_{\mathrm{mt}} \mathrm{O}_{2}$ used to generate the correction was altered from 65 to $75 \%$ (i.e. Correction II in Figure 7.4) and the SDS analysis re-applied. The mean regression coefficients again varied by less than the standard deviations on the mean values obtained for the fit with Correction I (Table 7.6).

The predicted chromophore concentrations for the in vivo tissue spectra will depend strongly on the assumed water content used to determine the other chromophore concentrations. Since the water content is essentially unknown, (although was assumed to be $60 \%$ for the WDPL), an upper and lower limit was determined. This was performed by comparing the regression coefficient for the water spectrum with previous measurements of the DP through the adult forearm at $800 \mathrm{~nm}$, the WDPL normalisation wavelength. Essenpreis et al (1993) determined a DPF of $4.48 \pm 0.41$ for the adult forearm using timeresolved spectroscopy, equating to a DP of $13.44 \pm 1.23 \mathrm{~cm}$ for a $3 \mathrm{~cm}$ separation. Fixing the upper and lower limits for the pathlength implied a range for the water concentration in the forearm, at an initial temperature of $37^{\circ} \mathrm{C}$, of $37-44.0 \%$. (It should be noted that varying the concentration of water used in the WDPL correction has little effect on the SDS-determined regression coefficients.) This range seems low compared to the value of $60 \%$ used for the WDPL. However, this value was based on the relative concentration of water in each compartment of the arm, i.e. skin, bone and muscle, and their relative concentrations in the arm itself (Matcher et al, 1994). Hence, it is an estimate of the mean water concentration in the arm and is likely to be incorrect since the arm is not homogeneous. In addition, the subject-to-subject variation of chromophore concentrations will be relatively high in the forearm due to the differences in the fat-to-muscle ratio (Matcher et al, 1994). The range implied by the SDS method is closer to the value found by Doornbos et al (1999) for the adult arm of approximately $47 \%$. The mean $\mathrm{Hb}$ concentrations inferred were 14.40 and $17.05 \mu \mathrm{Molar}$, for the assumption of 37 and $44 \%$ water content respectively.

The results of the SDS analysis were then used to estimate the underlying absorption 
spectrum of the initial set of tissue attenuation measurements (the 'calibration' measurement set), for the application of the non-linear optimisation routine to reconstruct the tissue absorption differences. Due to the large standard deviation of the $\mathrm{HbO}_{2}$ fit coefficients it was not possible to make a reasonable estimate of the $\mathrm{HbO}_{2}$ concentration using the SDS method, therefore it was estimated by assuming the $\mathrm{S}_{\mathrm{mt}} \mathrm{O}_{2}$. From the simulations of the Monte Carlo data in Section 7.2.1, it is clear that the estimate of the calibration $\mu_{a}$ will have a large effect on the $\mu_{a}$ differences predicted by the non-linear optimisation program. Hence two values for the $\mathrm{S}_{\mathrm{mt}} \mathrm{O}_{2}$ were assumed and the $\mathrm{HbO}_{2}$ concentrations were obtained from the SDS-estimated $\mathrm{Hb}$ concentrations for each $\mathrm{S}_{\mathrm{mt}} \mathrm{O}_{2}$. The two calibration absorption spectra generated, displayed in Figure 7.8, contain the following chromophore concentrations: $37 \%$ water, $14.4 \mu \mathrm{M} \mathrm{Hb}$ and $74 \% \mathrm{~S}_{\mathrm{mt}} \mathrm{O}_{2}$, i.e. $41.1 \mu \mathrm{M} \mathrm{HbO}_{2}$ (Set I), and $44 \%$ water, $17.1 \mu \mathrm{M} \mathrm{Hb}$ and $80 \% \mathrm{~S}_{\mathrm{mt}} \mathrm{O}_{2}$, i.e. $68.2 \mu \mathrm{M} \mathrm{HbO}_{2}$ (Set II). These combinations gave the maximum difference between the two absorption spectra, whilst varying the $\mathrm{S}_{\mathrm{mt}} \mathrm{O}_{2}$ and water concentration within the expected physiological range for the adult forearm.

The non-linear technique was applied to the difference attenuation, with respect to the first spectrum, of the in vivo forearm attenuation spectra. The transport scattering spectrum used was the spectrum also used in Section 7.1.3 for the SDS simulations and in
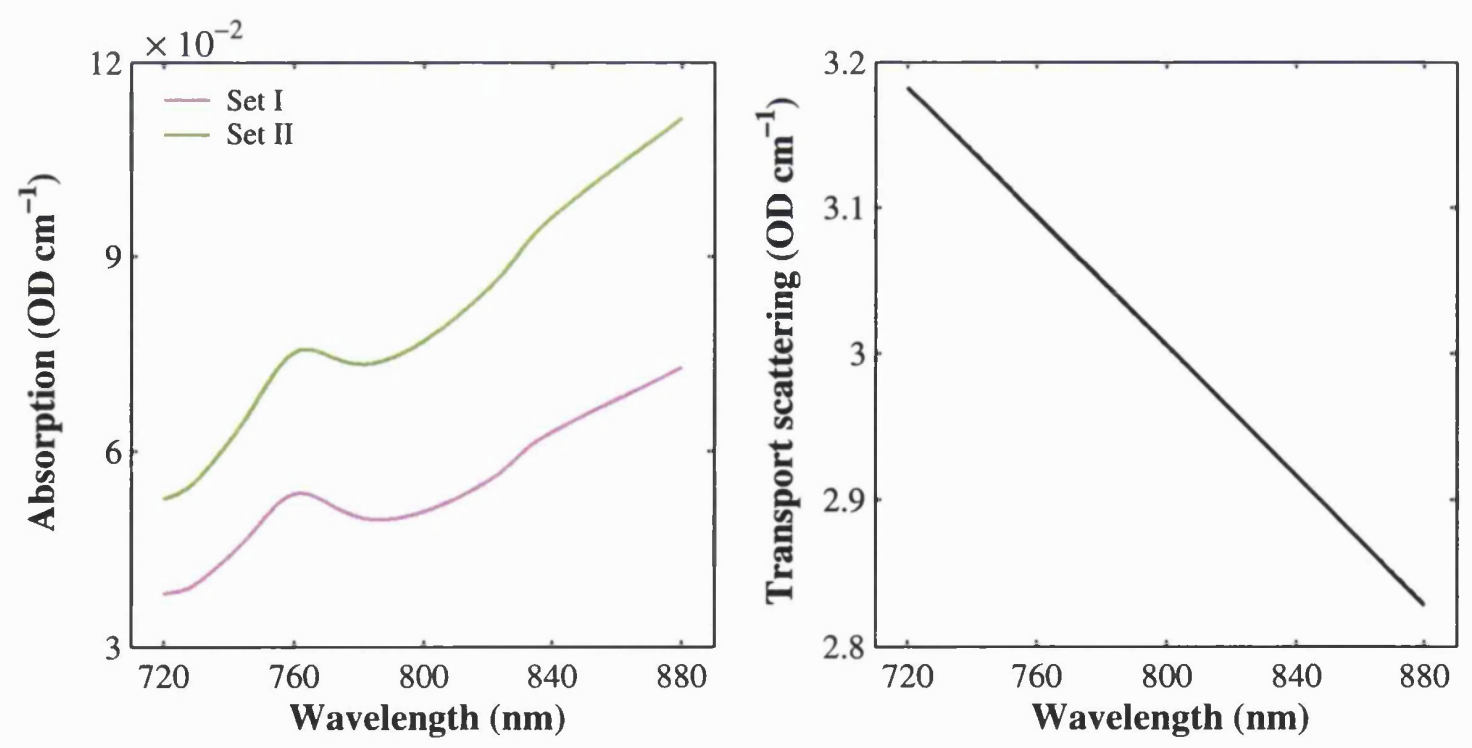

Figure 7.8: Calibration absorption and transport scattering spectra used in the reconstruction of the absorption differences from measurements of attenuation on the adult forearm using the Newton-Raphson optimisation routine. Left: the calibration absorption spectra for sets I and II of the chromophore concentrations (see text for details). Right: the transport scattering spectrum determined from the measurements of Matcher et al (1997) on the adult forearm. 


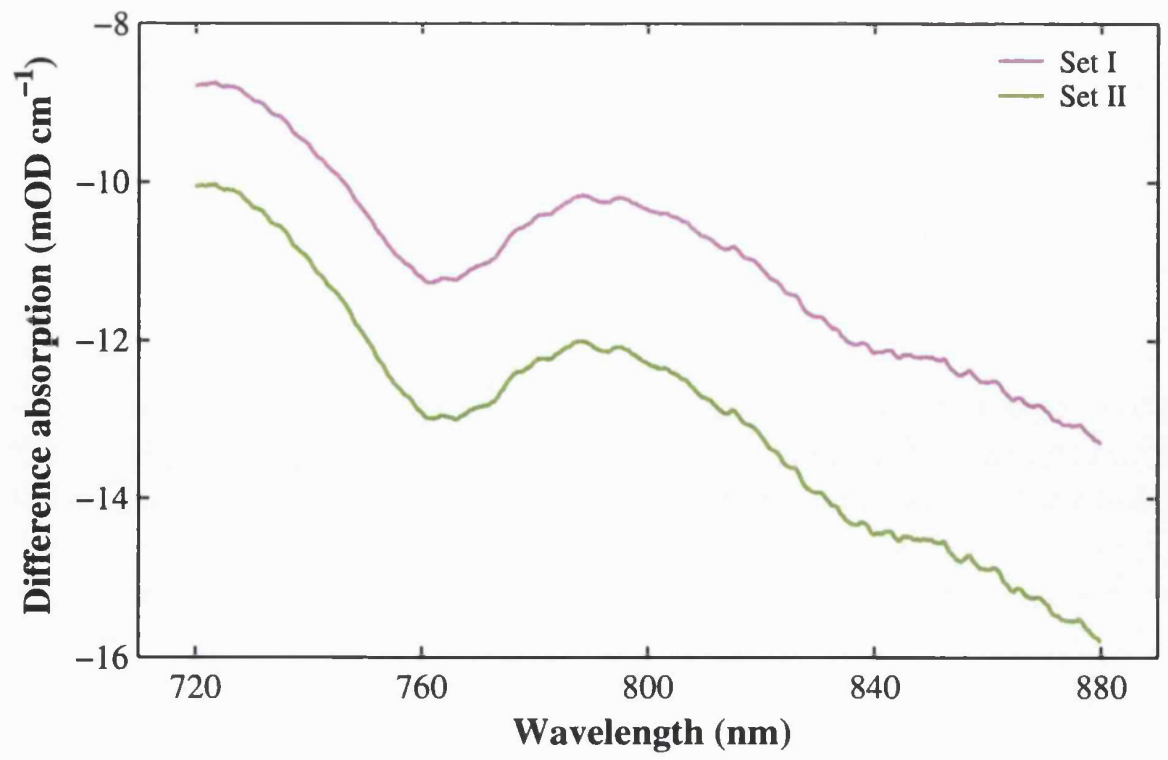

Figure 7.9: Final difference absorption spectra reconstructed from difference attenuation measurements of the adult forearm using the Newton-Raphson optimisation routine, for sets I and II (see text for details).

all the WDPL corrections (Matcher et al, 1997), displayed in Figure 7.8. The chromophore concentrations found by the above SDS analysis were used to estimate the calibration absorption spectrum, the underlying $\mu_{a}$ spectrum of the first attenuation measurement to which all difference attenuation spectra were referenced. The non-linear optimisation routine reconstructed each difference absorption spectrum in less than 20 iterations, using each spectrum at convergence as the estimate for the following measurement. Figure 7.9 shows the difference absorption spectra reconstructed by the non-linear method for the two sets of chromophore concentrations (Set I: $37 \%$ water, $74 \mathrm{~S}_{\mathrm{mt}} \mathrm{O}_{2}$; Set II: $44 \%$ water, $80 \mathrm{~S}_{\mathrm{mt}} \mathrm{O}_{2}$ ), in which the negative values indicate a decrease in absorption with cooling. As temperature decreases a decrease in absorption around the $760 \mathrm{~nm} \mathrm{Hb}$ absorption band and the water absorption band at $840 \mathrm{~nm}$ can be observed (changes in the $740 \mathrm{~nm}$ water absorption band are probably masked by the $760 \mathrm{~nm} \mathrm{Hb}$ band). The reconstruction of the difference absorption spectra will be discussed again in Section 7.2.4.

\subsubsection{Temperature Prediction}

The PCR calibration of the water absorption spectrum was then used to predict tissue temperature from the reconstruction of the tissue absorption spectra described in Section 7.2.2. As for the attempted SDS temperature prediction algorithm in Section 7.1.4, the components of the linear fit to the absorption spectra were the water loading vectors, 

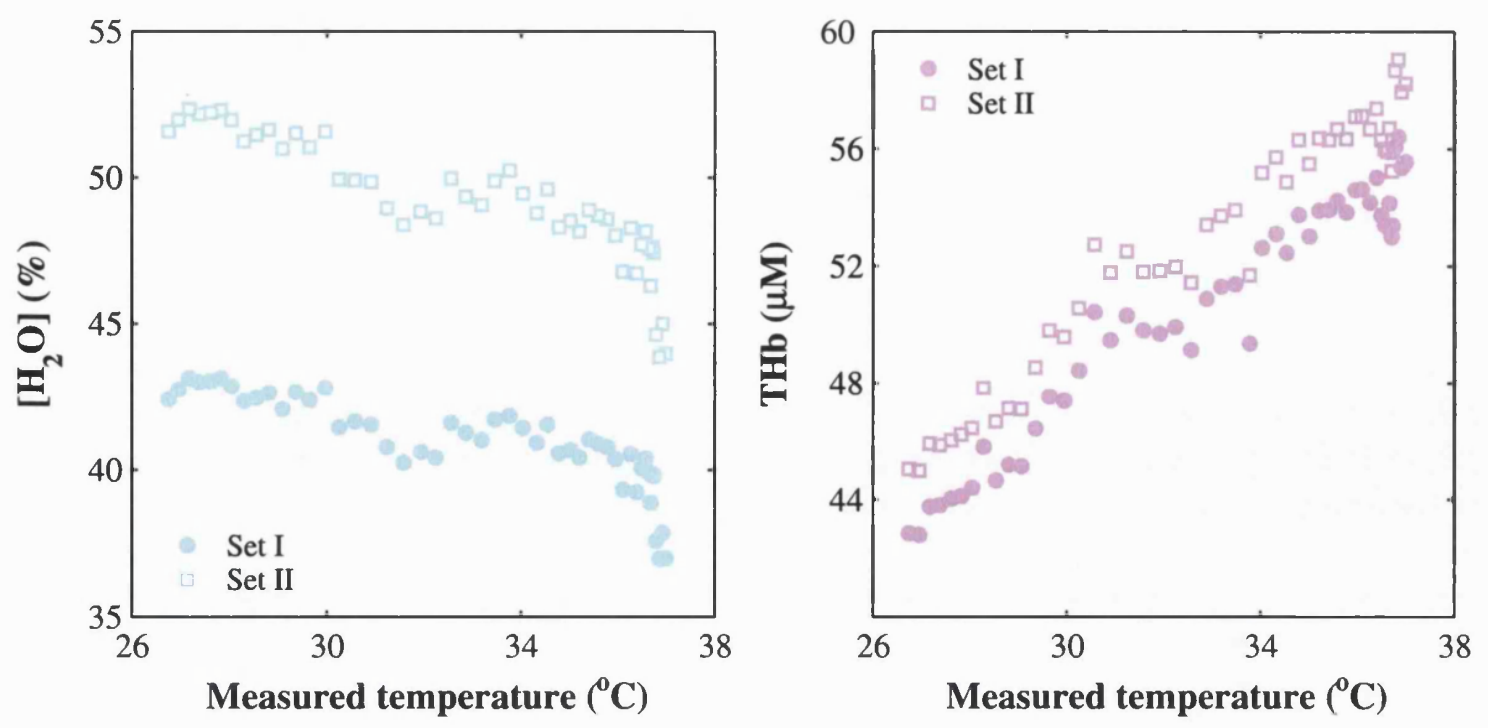

Figure 7.10: Chromophore concentrations as a function of temperature determined by a linear fit of the chromophore spectra to the reconstructed difference absorption spectra, for sets I and II. Left: the percentage water concentration. Right: the total haemoglobin concentration. Note that the concentrations displayed here are calculated from the sum of the concentrations obtained from the fits plus the assumed concentrations of the initial spectrum.

the mean water absorption spectrum and the $\mathrm{Hb}$ and $\mathrm{HbO}_{2}$ specific absorption spectra (except in the zero order). The fits to the absorption spectra were reasonable, the largest fit residuals occurring for the fit to the final absorption spectrum, as expected, in the order of $1 \%$ of the reconstructed $\mu_{a}$ values. Figure 7.10 shows the concentrations of water and total haemoglobin ( $\mathrm{THb}$ ) determined by the fits to the reconstructed absorption spectra, as a function of temperature. These 'total' concentrations are determined by adding the concentrations obtained from the fits to the assumed initial concentrations of the two sets (given in Section 7.2.2). The water concentration, determined from the fit of the mean water spectrum of the PCR calibration, can be seen to increase with decreasing temperature from 37 to $43 \%$ for Set I and from 44 to $52 \%$ for Set II over the whole temperature range $\left(37-27^{\circ} \mathrm{C}\right)$, as shown in the left-hand graph of Figure 7.10. However, as concomitant with a decrease in temperature, the concentrations of the loading vector (i.e. the scores) for the first PC of the water spectrum decrease with temperature by approximately $1 \%$ (not shown). The fact that the linear fit can determine an increase in the concentration of the mean water spectrum whilst at the same time a decrease in the scores of the first PC demonstrates that the two components are not co-linear, i.e. changes in total water concentration can be separated from changes in water temperature. The increase in water concentration can be explained if one considers that the volume of tissue probed by the light is likely to alter with temperature. As suggested in Section 
6.2.2, the average photon penetration depth will increase with decreasing temperature with the expected decrease in the optical coefficients. This means that the light will travel through a higher proportion of muscle tissue, which contains a much greater concentration of water than adipose tissue in the sub-dermal fat layer (Matcher et al, 1994). The total haemoglobin content $(\mathrm{THb})$ is seen to decrease with temperature by approximately 13 $\mu \mathrm{M}$ for both sets, as shown in the right-hand graph of Figure 7.10. As mentioned in Section 6.2.2, the decrease in the haemoglobin concentration with temperature may be due to physiological effects of the tissue cooling, since a decrease in temperature should actually increase the absorption by both $\mathrm{Hb}$ and $\mathrm{HbO}_{2}$ (Section 4.3.1.2). In Section 4.3.1.3 it was noted that the body's thermoregulatory responses to a decline in peripheral body temperature, such as vasoconstriction and the shunting of blood away from surface capillaries and vessels, could result in a decrease in the total amount of haemoglobin within the region probed by the light. Using a similar protocol to the one described in Section 6.2, Binzoni et al (1995) did in fact observe a significant change in blood perfusion and tissue blood volume at a temperature of approximately $36^{\circ} \mathrm{C}$. Although the THb decreased with temperature the $\mathrm{S}_{\mathrm{mt}} \mathrm{O}_{2}$ increased slightly ( $\sim 4 \%$ over the entire temperature range), i.e. $\mathrm{Hb}$ contributed more to the decrease in $\mathrm{THb}$ than did $\mathrm{HbO}_{2}$. This could be explained by the reduction in oxygen metabolism with decreasing temperature, which, as discussed in Sections 6.2.2 and 4.3.1.3, would increase the haemoglobin oxygen saturation in the blood vessels as less oxygen is consumed, and hence increases the $\mathrm{S}_{\mathrm{mt}} \mathrm{O}_{2}$. There is also a shift in the haemoglobin oxygen dissociation curve with temperature, which would further act to increase the $\mathrm{S}_{\mathrm{mt}} \mathrm{O}_{2}$ with decreasing temperature.

The tissue temperature was then predicted by multiplying the scores (corrected to $100 \%$ water by normalising to the concentration of the mean water spectrum, as described in Section 7.1.4) by the calibration vector of the PCR water calibration (see Section 5.3.5). Since the linear fitting of the water loading vectors (and tissue chromophores) is performed on the difference absorption spectra, it is the changes in temperature from the assumed baseline (i.e. $37^{\circ} \mathrm{C}$ ) that are predicted by this method. Figure 7.11 shows the predicted (changes plus baseline) versus the measured tissue temperature determined for the two sets of reconstructed absorption spectra, using only one PC from the PCR water calibration. The SEPs for tissue temperature prediction were 1.21 and $1.02{ }^{\circ} \mathrm{C}$ for sets I and II respectively, and the $r^{2}$ correlation coefficients were greater than 0.95 for both sets. Including further PCs in the fit to the absorption spectra improved the spectral fit but resulted in an extremely poor temperature prediction. Figure 7.11 shows that the 


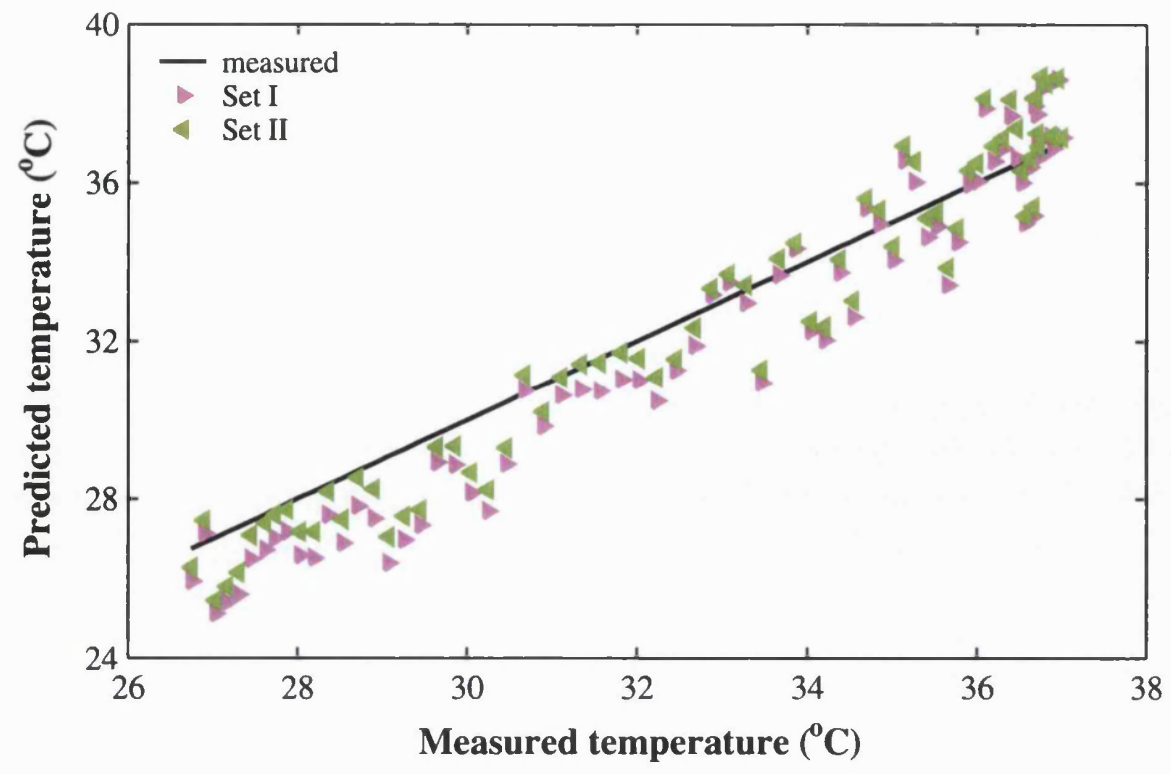

Figure 7.11: Predicted against measured tissue temperature as determined by applying the PCR calibration of the water spectrum to the reconstructed tissue absorption spectra, for sets I and II. The predicted temperatures are the changes in temperature determined from the difference absorption, plus the assumed baseline temperature of $37^{\circ} \mathrm{C}$

predicted temperatures increasingly underestimate the actual temperature (i.e. changes in temperature are overestimated) as the tissue temperature decreases. Interestingly the overestimation of the temperature using Set I is greater than for Set II, even though the difference $\mu_{a}$ (Figure 7.9) for Set I is smaller. These results will be discussed in further detail in the following section.

\subsubsection{Discussion}

A major assumption of the non-linear optimisation routine is that the transport scattering coefficient does not change with temperature. However, as discussed in Section 6.2, scattering within the volume of tissue probed is likely to decrease with cooling of the arm. A reduction in the actual $\mu_{s}^{\prime}$ of the tissue will cause the optimisation program to overestimate the absorption difference, due to the non-linear relationship between attenuation and $\mu_{a}$ and $\mu_{s}^{\prime}$. Figure 7.12 displays the attenuation against $\mu_{a}$, determined by the diffusion theory approximation in Equation (2.32), for several different values of $\mu_{s}^{\prime}$. In this example the measured attenuation difference $\triangle A$ is given by a decrease in $\mu_{s}^{\prime}$ from point 1 to point 2 (see Figure 7.12) and a decrease in $\mu_{a}$ from point 2 to point 3, i.e. $\mu_{a}$ decreases from $\mu_{a c a l}$ by $\Delta \mu_{a}$. If the change in $\mu_{s}^{\prime}$ is underestimated, or in this case completely ignored, in order to obtain an equivalent $\triangle A$ the attenuation must change between points 1 and 4 (at the same $\mu_{s}^{\prime}$ value), in which case the reconstructed difference 


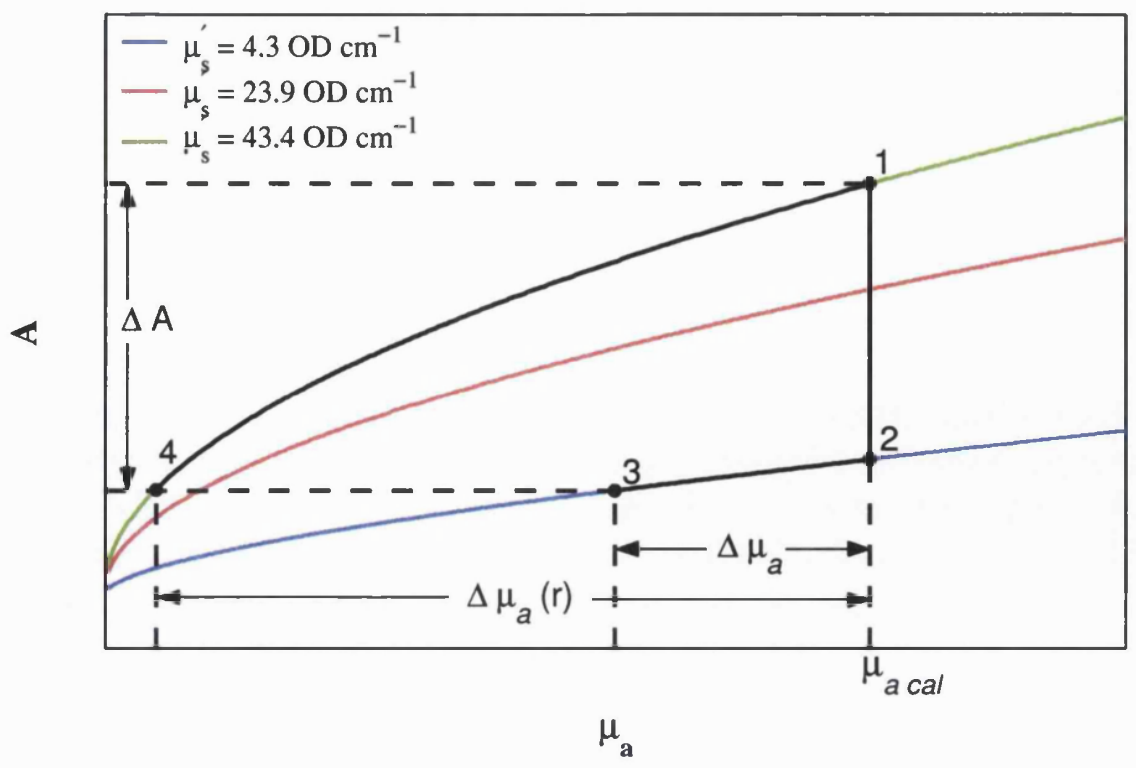

Figure 7.12: The effect of underestimating the change in scattering upon the reconstructed difference absorption, see text for details.

absorption, $\triangle \mu_{a}(\mathrm{r})$, is significantly overestimated compared to the true change $\triangle \mu_{a}$. Using the Monte Carlo simulations of Section 7.2.1, the optimisation program was applied to the difference in attenuation calculated from simulations IV and I (see Table 7.5). In this case $\mu_{a}$ decreased by $0.043 \mathrm{OD} \mathrm{cm}^{-1}$ and $\mu_{s}^{\prime}$ decreased by $2.17 \mathrm{OD} \mathrm{cm}^{-1}$, although the input $\mu_{s}^{\prime}$ was fixed at the higher value of $6.51 \mathrm{OD} \mathrm{cm}^{-1}$. With an input estimate of $-0.043 \mathrm{OD} \mathrm{cm}^{-1}$ for $\Delta \mu_{a}$ (the correct value) and the actual value of $\mu_{a \text { cal }}$ (the $\mu_{a}$ value to which the change in absorption is referenced), the difference absorption returned was $-0.0615 \mathrm{OD} \mathrm{cm}^{-1}$, an overestimation of $\sim 42 \%$. Although the change in $\mu_{s}^{\prime}$ is approximately ten times larger than those reported in the literature for temperature-dependent changes in tissue, i.e. $5 \%{ }^{\circ} \mathrm{C}^{-1}$ compared to $\sim 0.5 \%{ }^{\circ} \mathrm{C}^{-1}$ (Laufer et al, 1998; Yeh et al, 2001), the simulation demonstrates the effect of assuming an unchanging $\mu_{s}^{\prime}$ spectrum on the absorption differences reconstructed by the non-linear routine. The overestimation of the difference absorption will lead to an overestimation of the change in temperature with decreasing temperature, as shown in Figure 7.11.

To examine whether accounting for the likely change in $\mu_{s}^{\prime}$ with temperature would improve the prediction of tissue temperature by this method, changes in $\mu_{s}^{\prime}$, simulated using the results of Yeh et al (2001), were incorporated into the non-linear optimisation program. For the adult forearm Yeh et al determined a $\mu_{s}^{\prime}$ in the range $3.9-4.8 \mathrm{OD} \mathrm{cm}^{-1}$ at $590 \mathrm{~nm}$ and $2.4-2.8 \mathrm{OD} \mathrm{cm}^{-1}$ at $950 \mathrm{~nm}$. The changes in $\mu_{s}^{\prime}$ were $0.30 \pm 0.09$ and $0.14 \pm 0.08 \mathrm{OD} \mathrm{cm}^{-1}$ at 590 and $950 \mathrm{~nm}$ respectively. These results give an upper and 


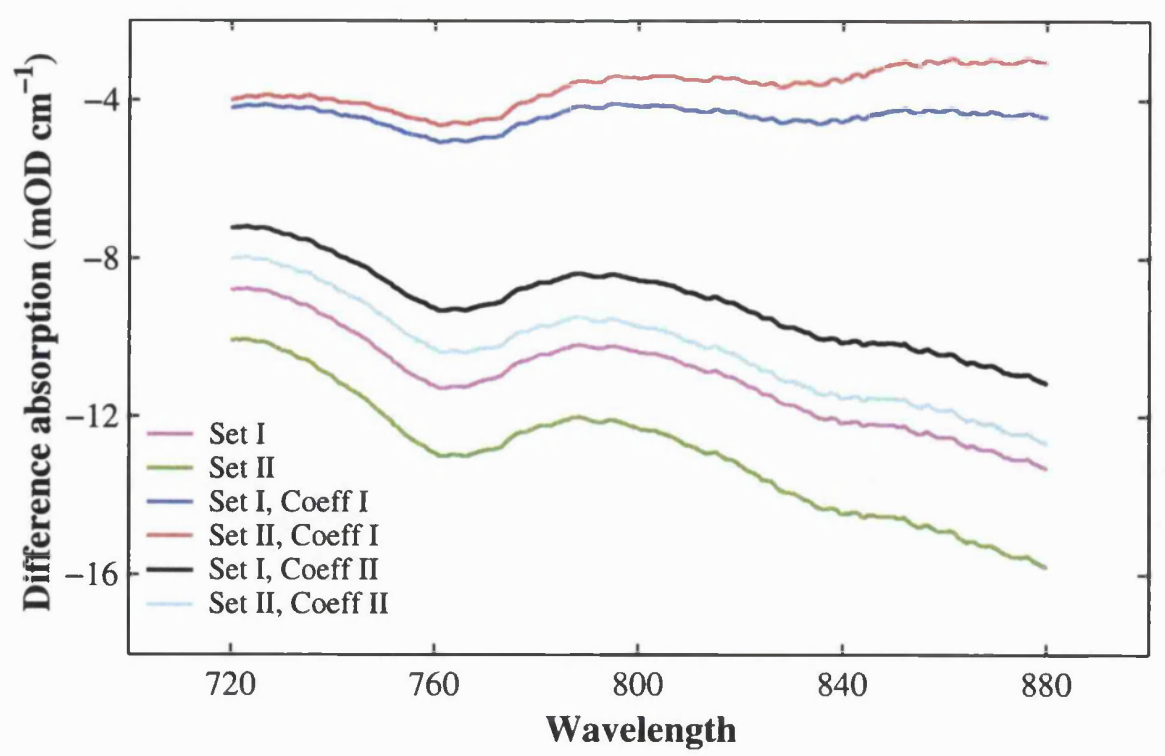

Figure 7.13: Final difference absorption spectra reconstructed from difference attenuation measurements of the adult forearm incorporating simulated changes in the transport scattering spectrum with temperature, for sets I and II and temperature coefficients I and II (see text for details).

lower limit for the temperature coefficient of $\mu_{s}^{\prime}: 0.27-0.61 \%{ }^{\circ} \mathrm{C}^{-1}$ at $590 \mathrm{~nm}$ and 0.13 $0.59 \%{ }^{\circ} \mathrm{C}^{-1}$ at $950 \mathrm{~nm}$. Combining the upper and lower limits respectively, two sets of temperature coefficients for $\mu_{s}^{\prime}$ were simulated by linearly interpolating the values at 590 and $950 \mathrm{~nm}\left(0.61\right.$ and $0.59 \%{ }^{\circ} \mathrm{C}^{-1}$ for Coefficient $\mathrm{I}$ and 0.27 and $0.13 \%{ }^{\circ} \mathrm{C}^{-1}$ for Coefficient II) to the range $720-880 \mathrm{~nm}$. Although the measurements of Yeh et al (2001) were performed using a much smaller source-detector separation than the current study, and will therefore sample mostly dermis as opposed to muscle tissue, the method described above for calculating the the temperature coefficients of $\mu_{s}^{\prime}$ gives a reasonably wide range of values, which, in the absence of any other in vivo temperature coefficients, will at least serve to demonstrate the effect of incorporating different values of $\Delta \mu_{s}^{\prime}$ on the temperature prediction. The simulated changes in $\mu_{s}^{\prime}$ were obtained by applying the two temperature coefficients to the original $\mu_{s}^{\prime}$ spectrum (Figure 7.8) at each measured tissue temperature. Instead of fixing the $\mu_{s}^{\prime}$ spectrum, the non-linear optimisation program then incorporated the changes in $\mu_{s}^{\prime}$ into the reconstruction of the $\Delta \mu_{a}$ spectra. Figure 7.13 shows the final difference absorption spectra determined using the same two $\mu_{a \text { cal }}$ spectra for each $\mu_{s}^{\prime}$ temperature coefficient. The original $\Delta \mu_{a}$ spectra, determined for the fixed $\mu_{s}^{\prime}$, are included for comparison. For both of the simulated temperature coefficients the reconstructed difference absorption is smaller than for the original reconstruction in which the $\mu_{s}^{\prime}$ did not alter with temperature. As expected, the temperature coefficient that gives the larger change in $\mu_{s}^{\prime}$ (Coefficient I) returns the smallest difference absorption. Moreover, the 


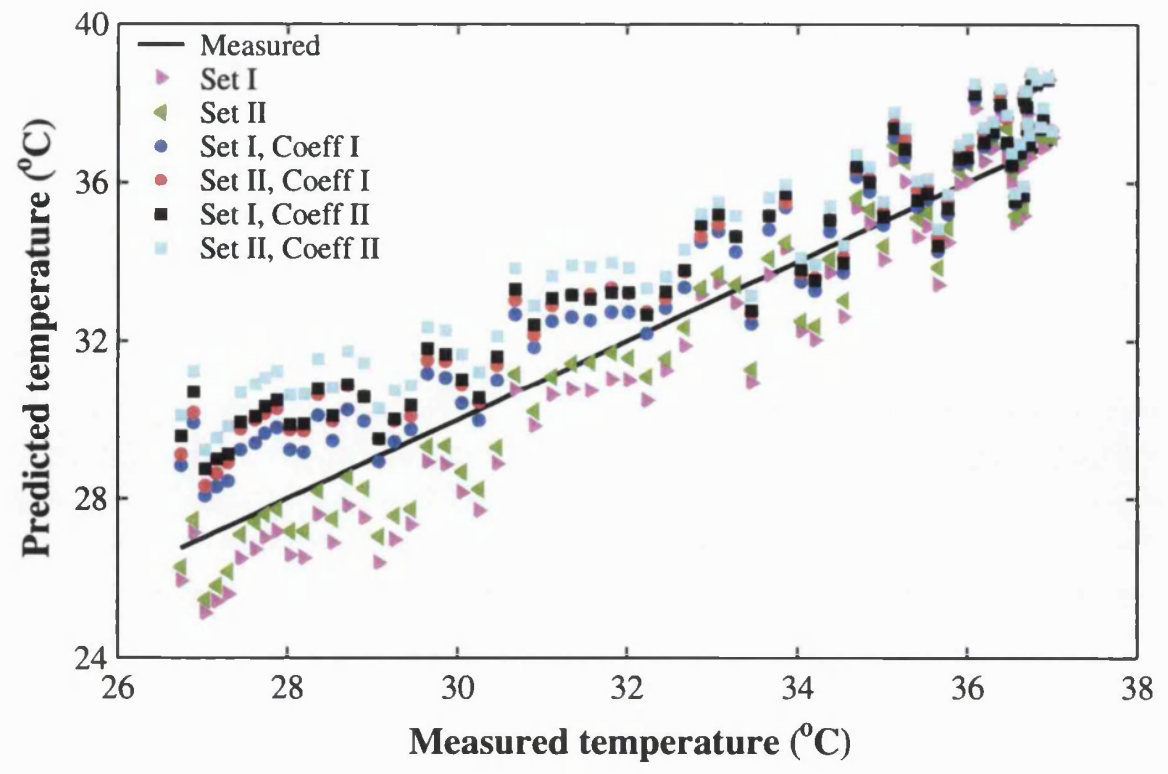

Figure 7.14: Predicted against measured tissue temperature for absorption reconstructions incorporating a decrease in the transport scattering coefficient with temperature, for sets I and II and temperature coefficients I and II (see text for details)

wavelength-dependence of the $\Delta \mu_{a}$ reconstructed with the two temperature coefficients is quite different. The $\Delta \mu_{a}$ spectra reconstructed with Coefficient I exhibit a smaller $\Delta \mu_{a}$ at longer wavelengths relative to those reconstructed with Coefficient II. This is due to the different wavelength-dependencies of the temperature coefficients. Coefficient I hardly varies within the range of interest, therefore $\triangle \mu_{a}$ will be lower at wavelengths where the values of the initial $\mu_{a \text { cal }}$ spectrum were large, i.e. at longer wavelengths (see Figure 7.8). Coefficient II has a strong wavelength-dependence in comparison, the values of $\Delta \mu_{s}^{\prime}$ being lower at longer wavelengths, which will increase the reconstructed $\Delta \mu_{a}$ relative to the shorter wavelengths.

The changes in tissue temperature were predicted for each $\triangle \mu_{a}$ set using the method described in Section 7.2.3. The linear fitting of the chromophore and water loading spectra to the new reconstructed $\triangle \mu_{a}$ spectra revealed slightly smaller spectral residuals than for the reconstructions in which no $\Delta \mu_{s}^{\prime}$ was simulated. Figure 7.14 shows the actual versus predicted temperature for each reconstruction, including the original (fixed $\mu_{s}^{\prime}$ ) values for comparison. The changes in tissue temperature are now underestimated (i.e. the total temperature is overestimated) by simulating changes in the transport scattering coefficient with temperature. Interestingly, the reconstruction with $\mu_{a \text { cal }}$ and $\Delta \mu_{s}^{\prime}$ generated from Set II and Coefficient II respectively returns a (final) difference $\mu_{a}$ that is most similar to the original sets (see Figure 7.13), yet gives a temperature prediction that is furthest from those of the original reconstructions. Moreover the converse is true: the reconstructions 


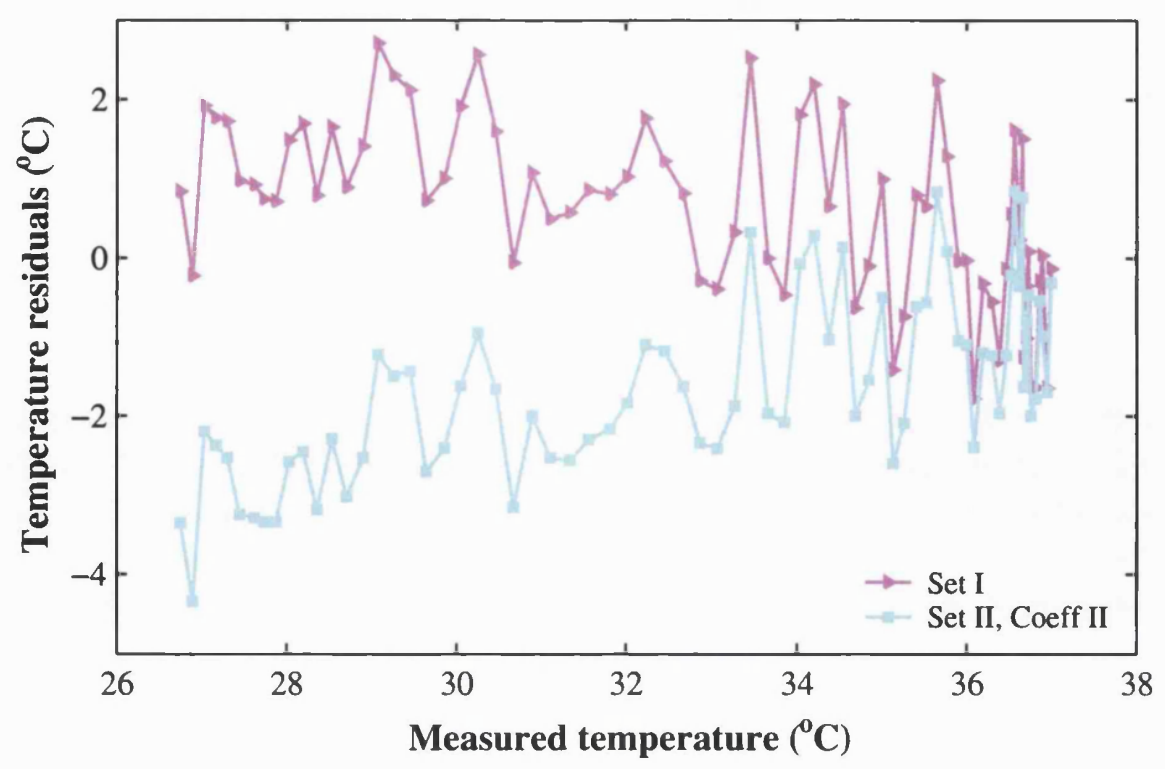

Figure 7.15: Temperature residuals for reconstructions using Set I and Set II with Coefficient II. Note the change in the temperature prediction at approximately $34^{\circ} \mathrm{C}$ for both reconstructions.

that are most different in their $\Delta \mu_{a}$ spectra give the most similar temperature prediction. This suggests that the wavelength-dependence of the $\mu_{s}^{\prime}$ temperature coefficient is more important than its absolute magnitude in terms of temperature prediction.

By adjusting the magnitude and wavelength-dependence of the $\mu_{s}^{\prime}$ temperature coefficients somewhat arbitrarily it was possible to improve the temperature prediction slightly. Similar improvements were also obtained by altering the input $\mu_{a}$ and $\mu_{s}^{\prime}$ calibration spectra, to which the changes are referenced. However, in all cases the predicted temperature changes were seen to alter (depending on the $\mu_{s}^{\prime}$ temperature coefficients employed) at around $34^{\circ} \mathrm{C}$. This is shown in Figure 7.15 by the temperature residuals (actual - predicted) for the reconstructions using Set I and no $\Delta \mu_{s}^{\prime}$, and Set II and Coefficient II. It is possible that at around $34^{\circ} \mathrm{C}$, a change in blood volume due to vasoconstriction in the volume of tissue interrogated by the light causes a significant change in $\mu_{s}^{\prime}$ and/or its temperature coefficient, which is not modelled by the optimisation routine. Hence, regardless of the magnitude and wavelength-dependence of the $\mu_{s}^{\prime}$ temperature coefficients, this non-linearity in the change in $\mu_{s}^{\prime}$ with temperature is not accounted for.

Another important point to note is that the temperature prediction by this method (for any given reconstruction) is not improved by the inclusion of more water loading vectors, i.e. more principal components, which should describe a greater percentage of the variance in the water data. Since the additional loading vectors will make such a relatively small contribution to the total absorption spectra, it is possible that the numerical process of 
the least-squares fit will produce such large errors on their estimated concentrations that their inclusion will significantly degrade the temperature prediction, as was the case in the second derivative (Section 7.1.4). To determine if this was the case, fits to a set of $\mu_{a}$ spectra (generated for the test of the SDS temperature prediction algorithm in Section 7.1.4), in which only the temperature-dependence of the water spectrum contributed to changes in $\mu_{a}$, were performed for the inclusion of one to four PCs. Random noise was included on the spectra in the range $0-0.1 \%$ of the total $\mu_{a}$ at each wavelength. In all cases the inclusion of more PCs reduced the spectral fit residuals and reduced the slight non-linearity in the temperature residuals. As the noise increased above $0.05 \%$, though, the inclusion of more PCs increased the SEC due to the greater significance of the noise on the smaller loading vectors, which counteracted the improvement in the SEC due to a decrease in the non-linearity of the temperature residuals. This still does not explain, however, the extremely large and temperature-dependent overestimation of the temperature, (approximately $18-35^{\circ} \mathrm{C}$ over the whole temperature range), when including a second PC in the fit to the tissue absorption spectra. Another possible explanation, therefore, could be that the additional loading vectors are attempting to account for the non-linearity produced by the amplitude change (and peak position shift) in the $760 \mathrm{~nm} \mathrm{Hb}$ band with decreasing temperature (see Section 4.3.1.2). With increasing temperature in the range $20-40^{\circ} \mathrm{C}$ the amplitudes of the $\mathrm{Hb}$ and $\mathrm{HbO}_{2}$ bands in the NIR have been shown to increase by approximately 0.15 and $<0.05^{\circ} \mathrm{C}^{-1}$ respectively (Sfareni et al, 1997). The amplitude changes in $\mathrm{Hb}$ are likely to have a more significant effect on the total absorption in the range of interest than those for $\mathrm{HbO}_{2}$, since the former are larger and the $\mathrm{Hb}$ peak at $760 \mathrm{~nm}$ is more defined than the broad $\mathrm{HbO}_{2}$ band (centered at approximately 930 $\mathrm{nm})$. Although the percentage change in $\mathrm{Hb}$ absorption per ${ }^{\circ} \mathrm{C}$ is smaller than for water at $760 \mathrm{~nm},\left(\sim 0.15 \%{ }^{\circ} \mathrm{C}^{-1}\right.$ for $\mathrm{Hb}$ and $0.5 \%{ }^{\circ} \mathrm{C}^{-1}$ for water $)$, their relative contributions to the total absorption coefficient at $760 \mathrm{~nm}$ are approximately 8 and $45 \%$ respectively for the Set I $\mu_{a c a l}$ spectrum, (37\% water, $\left.74 \% \mathrm{~S}_{\mathrm{mt}} \mathrm{O}_{2}\right)$, and approximately 7 and $38 \%$ respectively for the Set II $\mu_{a \text { cal }}$ spectrum, (44\% water, $\left.80 \% \mathrm{~S}_{\mathrm{mt}} \mathrm{O}_{2}\right)$. Hence the effect of the temperature-induced $\mathrm{Hb}$ changes in the forearm could be comparable to, if not larger than, the effect of the changes in the water spectrum, around $760 \mathrm{~nm}$ (see Chapter 8 for further discussion). 


\subsection{Algorithm III: Spatially-Resolved Reflectance Method}

The main disadvantage of the algorithm described in Section 7.2 is the inability of the non-linear optimisation program to determine changes in both $\mu_{a}$ and $\mu_{s}^{\prime}$ with temperature. The transport scattering coefficient must be fixed, or its temperature coefficient estimated, both of which will lead to errors in the temperature prediction if the magnitude or wavelength-dependence of either $\mu_{s}^{\prime}$ or its temperature coefficient change significantly with temperature (see Section 7.2.4). Algorithm III therefore attempts to resolve both $\mu_{a}$ and $\mu_{s}^{\prime}$ changes with temperature using measurements made at multiple source-detector separations.

The experiment described in Section 6.3 involves the spatially-resolved measurement of a tissue-like phantom consisting of Intralipid- $10 \%^{\mathrm{TM}}$, isotonic saline and an absorbing dye (copper sulphate). As discussed briefly in Section 6.3, by measuring at multiple source-detector separations on the surface of a medium, one should be able to determine absolute values of both $\mu_{a}$ and $\mu_{s}^{\prime}$ by comparing the measurements with a model of the spatially-resolved reflectance from such a medium. Based on a diffusion theory solution for the diffuse reflectance from a semi-infinite turbid medium (see Section 2.3), Nichols et al (1997) were able to determine the optical coefficients of various tissue phantoms to an accuracy of within 5-15\%, from measurements of reflectance at fifteen source-detector separations in the range $0.1-2 \mathrm{~cm}$. In fact, they determined the optical coefficients from relative reflectance values, i.e. the spatially-resolved reflectances normalised to the reflectance at one known source-detector separation. The process of normalising the experimentallydetermined (and modelled) reflectances removes the necessity for absolute reflectance measurements, which depend on such factors as the incident intensity and the diameters and numerical apertures of the fibres (Farrell and Patterson, 1992).

It seems, therefore, that reasonable estimations of absolute $\mu_{a}$ and $\mu_{s}^{\prime}$ values can be determined from the wavelength-dependence of spatially-resolved measurements. However, as noted by Nichols et al (1997), and other workers (Farrell and Patterson, 1992; Hull et $a l, 1998)$, in order to accurately resolve $\mu_{a}$ and $\mu_{s}^{\prime}$ the reflectance measurements must include both small and large source-detector separations, estimated by Nichols et al to be in the range of $0.75-1$ to $10-20$ transport mean free paths $\left(\mu_{t}^{\prime-1}\right)$ respectively. This is because the reflectance at small source-detector separations is dominated by $\mu_{s}^{\prime}$, whereas at larger separations the effective attenuation coefficient $\mu_{\text {eff }}$ is dominant (see Section 2.3). Since $\mu_{\text {eff }}$ contains the product of $\mu_{a}$ and $\mu_{s}^{\prime}$, fitting to the reflectance measured at large 
separations alone will not uniquely determine the optical coefficients. Based on the values of $\mu_{a}$ and $\mu_{s}^{\prime}$ employed in the tissue phantom described in Section 6.3, i.e. typical values for biological tissue, the source-detector separations should range from a minimum of around $0.1 \mathrm{~cm}$ up to about $1-2 \mathrm{~cm}$, if the coefficients are to be determined using the method of Nichols et al (1997). At this range of distances, however, the average penetration depth (see Section 2.3) from the surface of the medium will vary between approximately 0.1 and $0.5 \mathrm{~mm}$. Whilst this degree of penetration may be suitable for probing relatively small volumes of tissue close to the surface, e.g. superficial tumours (Hull et al, 1998), it would not be sufficient to interrogate deeper tissues such as brain and muscle, tissues that are of particular relevance to the work of this project. If additional measurements at greater source-detector separations were included, such that the deeper tissue was also probed, the optical coefficients determined would in any case be an average of all the tissues in the volume probed. Due to the heterogeneity of the tissues involved (see Section 3.3), one could not expect these (weighted) averages to represent the optical properties of the tissue of interest. For this reason the fibre separations for the spatially-resolved measurements of the tissue phantom (Section 6.3) were chosen to be in the range 1.8-3.6 cm, i.e. $18-36$ transport mean free paths, for which the average photon depth is approximately $0.5-0.7$ $\mathrm{cm}$, capable of penetrating deeper tissues without including significant contributions from surface tissues (see Section 3.1.3). According to the studies discussed here (Farrell and Patterson, 1992; Hull et al, 1998; Nichols et al, 1997), these source-detector separations will not provide sufficient uniqueness to the solution to resolve absolute values of both $\mu_{a}$ and $\mu_{s}^{\prime}$. Although these findings were duly noted, an attempt was made to reconstruct the absorption and transport scattering coefficients from the spatially-resolved measurements of the tissue phantom as a function of temperature (Section 6.3) by this method, since it may transpire that whilst the absolute coefficients are poorly determined, the difference values (i.e. with decreasing temperature) are reasonably well preserved.

The spatially-resolved measurements of the tissue phantom described in Section 6.3 were normalised to the measurement at $1.8 \mathrm{~cm}$ and compared to the diffusion theory solution for spatially-resolved reflectance from a semi-infinite turbid medium, derived using the extrapolated boundary condition (see Section 2.3). The reflectance $R(\rho)$ is defined as the fraction of incident intensity $I_{0}$ detected on the surface of the medium at some distance $\rho$ from the source. Since the incident intensity is generally difficult to measure (see Section 3.1), the intensity measured at the exit port-hole of an integrating sphere (Section 6.3), 
which is proportional to the incident intensity, is used to calculate the reflectance as follows

$$
R(\rho)=\frac{I_{\mathrm{P}}(\rho)}{I_{\mathrm{IS}}(\rho)}
$$

where $I_{\mathrm{P}}(\rho)$ is the intensity measured a distance $\rho$, from the source on the surface of the phantom and $I_{\mathrm{IS}}(\rho)$ is the corresponding integrating sphere intensity measurement. Although the latter is not spatially-resolved, this reference measurement must be performed for each fibre in order to account for its individual characteristics. The reflectance at each source-detector separation, calculated using Equation (7.13), was normalised to the reflectance measured at the smallest separation of $1.8 \mathrm{~cm}$. The log normalised reflectances were then fitted to the diffusion theory expression (also $\log$ normalised to $\rho=1.8 \mathrm{~cm}$ ) using a non-linear least-squares fitting routine based on the Levenberg-Marquardt method (Press et al, 1992). The log of the reflectance was used since it is more robust than the reflectance data itself (Kienle and Patterson, 1997). Figure 7.16 shows the log normalised reflectance at three source-detector separations calculated from diffusion theory, Monte Carlo simulations (Okada, 2002) and the experimental measurements of the tissue phantom. All are normalised to the smallest separation of $1.8 \mathrm{~cm}$. In the left-hand graph of Figure 7.16 , the $\log$ normalised reflectance generated for a $\mu_{a}$ of $0.043 \mathrm{OD} \mathrm{cm}^{-1}$ and a $\mu_{s}^{\prime}$ of $4.34 \mathrm{OD} \mathrm{cm}^{-1}$ using the diffusion theory solution of Equation (2.31) is compared
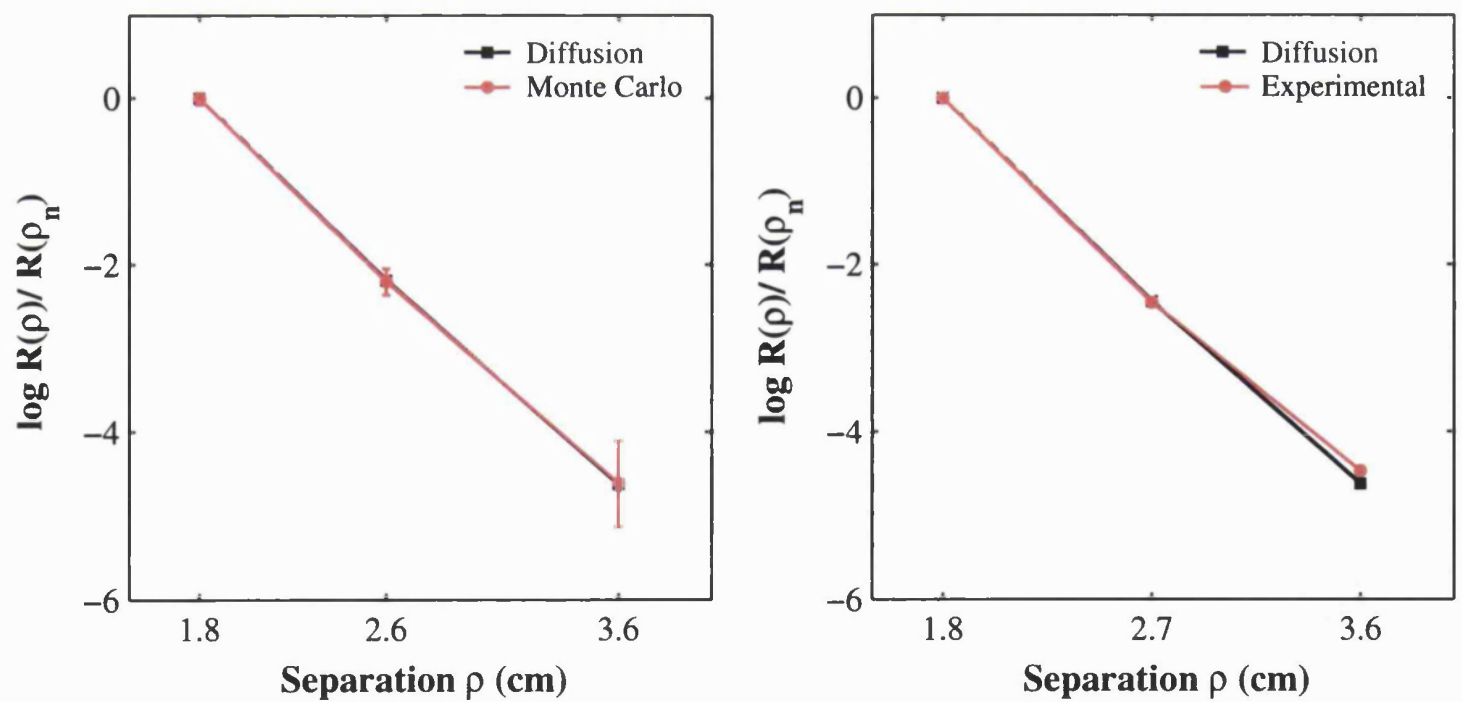

Figure 7.16: Log normalised spatially-resolved reflectance generated using diffusion theory, Monte Carlo simulations and experimental measurements of a tissue phantom, at three source-detector separations between 1.8 and $3.6 \mathrm{~cm}$. Left: Monte Carlo simulations and diffusion theory solution of reflectance for $\mu_{a}$ and $\mu_{s}^{\prime}$ of 0.043 and $4.34 \mathrm{OD} \mathrm{cm}^{-1}$. Right: Experimentally-determined and diffusion theory reflectances for $\mu_{a}$ and $\mu_{s}^{\prime}$ of 0.045 and $4.21 \mathrm{OD} \mathrm{cm}^{-1}$ (see text for details). 
to that (with the same optical coefficients) determined from the Monte Carlo simulations first described in Section 7.2.1, at separations of 1.8, 2.6 and $3.6 \mathrm{~cm}$ (note that only evennumbered separations were used in the MC simulations). The error bars on the Monte Carlo reflectances are calculated assuming Poisson-noise on the intensity values (Section 3.1 , with an input of 10 million photons. In the right-hand graph, the experimentallydetermined reflectances measured at $1.8,2.7$ and $3.6 \mathrm{~cm}$ are displayed for a $\mu_{a}$ and $\mu_{s}^{\prime}$ of 0.045 and $4.21 \mathrm{OD} \mathrm{cm}^{-1}$ respectively, the estimated values of the coefficients at $800 \mathrm{~nm}$ (see Figure 7.8). These are compared to the diffusion theory solution for the same optical properties. Uncertainties on the experimental data were also estimated (see Appendix A), although these are small due to the averaging of the data, and the error bars are obscured by the data points. It should be noted here that although the centres of the source and detecting fibres are separated by $1.8,2.7$ and $3.6 \mathrm{~cm}$ for the three measurements respectively, the light reaching the the detecting fibres is most intense (i.e. less attenuated) at the edge closest to the source, hence the true (average) separation is weighted away from the centre in the direction of the source fibre. Moreover, the fibre bundles (see Section 6.3) are not specifically aligned at the spectrometer end, such that the bundles illuminating the spectrometer slit may not represent a uniform distribution of bundle positions on the surface of the phantom, which will have an additional weighting effect on the average separation. This may explain the discrepancies $(\sim 3.5 \%$ at $3.6 \mathrm{~cm})$ between the experimentally-determined and diffusion theory reflectances, since in the latter a point source is assumed to illuminate the medium (see Section 2.3).

The agreement between the diffusion theory model and the simulated or measured reflectances, displayed in Figure 7.16, is reasonable. However, for both the simulated and measured reflectances, the fit to the model, using the Levenberg-Marquardt non-linear least-squares fitting routine, could not converge to a solution unless the tolerances for changes in both the objective function (the difference between the input and the model) and the optical coefficients were suitably large, rendering the sensitivity of the technique, i.e. its ability to determine real changes in the optical coefficients, extremely poor. Increasing the range and number of source-detector separations included in the fit of the Monte Carlo reflectances resulted in convergence, but the parameters returned were highly dependent on the input estimates of the optical coefficients, demonstrating the non-uniqueness of the problem noted previously. Therefore, a rigorous analysis of the ability of the method to accurately determine differences in $\mu_{a}$ and $\mu_{s}^{\prime}$ was not performed. Instead, a linear approximation of the diffusion theory solution for the spatially-resolved reflectance was 
investigated. The theory underlying this approximation, and its application to determine $\mu_{a}$ and $\mu_{s}^{\prime}$ from measurements at large source-detector separations, is discussed in the following section.

\subsubsection{Theory}

The linear approximation of the diffusion theory solution to the spatially-resolved reflectance is based on the fact that at large-source detector separations $\left(>10-20 \mu_{t}^{-1}\right)$, the reflectance varies almost linearly with distance from the source (Liu et al, 1995). Using the diffusion theory solution for the spatially-resolved reflectance from a semi-infinite medium defined in Equation (2.32), i.e. derived from the zero boundary condition, the intensity measured at distance $\rho$ from the source on the surface of the medium is given by

$$
I(\rho)=\frac{I_{0}}{2 \pi \mu_{t}^{\prime}}\left(\mu_{e f f}+\frac{1}{r}\right) \frac{\exp \left(-\mu_{e f f} r\right)}{r^{2}}
$$

where $I_{0}$ is the incident intensity and $r$ is the distance from the observation point $\rho$ to the isotropic point source at $1 / \mu_{t}^{\prime}$ below the surface (see Section 2.3). At distances far from the source, i.e. $\rho \gg \mu_{t}^{-1}$ hence $r \approx \rho$, the following approximation can be made

$$
I_{a}(\rho)=\frac{1}{c \mu_{t}^{\prime}}\left(\mu_{e f f}+\frac{1}{\rho}\right) \frac{\exp \left(-\mu_{e f f} \rho\right)}{\rho^{2}}
$$

where $c=2 \pi / I_{0}$. Rearranging the expression for the approximated intensity $I_{a}$ and taking logs of both sides gives

$$
\log _{10}\left(\rho^{2} I_{a}(\rho)\right)=-\log _{10}\left(c \mu_{t}^{\prime}\right)+\log _{10}\left(\mu_{e f f}+\frac{1}{\rho}\right)-\frac{\mu_{e f f} \rho}{2.3}
$$

The second term of Equation (7.16) is non-linear with respect to $\rho$ but can be approximated by $\log _{10}\left(\mu_{\text {eff }}+1 / \rho_{0}\right)$, as demonstrated by Liu et al (1995), where $\rho_{0}$ is the mid-point of the minimum and maximum source-detector separations. Thus, the expression for the spatially-resolved intensity is linearised with respect to the source-detector separation $\rho$ using the following approximation

$$
\log _{10}\left(\rho^{2} I_{a}\left(\rho, \rho_{0}\right)\right)=-\log _{10}\left(c \mu_{t}^{\prime}\right)+\log _{10}\left(\mu_{\text {eff }}+\frac{1}{\rho_{0}}\right)-\frac{\mu_{\text {eff }} \rho}{2.3}
$$

Figure 7.17 displays the percentage error of the approximated expression in Equation (7.17) relative to the exact solution in Equation (7.14), i.e. the error in $\log _{10}\left(\rho^{2} I_{a}\left(\rho, \rho_{0}\right)\right)$ compared to $\log _{10}\left(\rho^{2} I(\rho)\right)$, for an input intensity of $1, \mu_{a}$ and $\mu_{s}^{\prime}$ of 0.043 and $4.34 \mathrm{OD} \mathrm{cm}^{-1}$ 


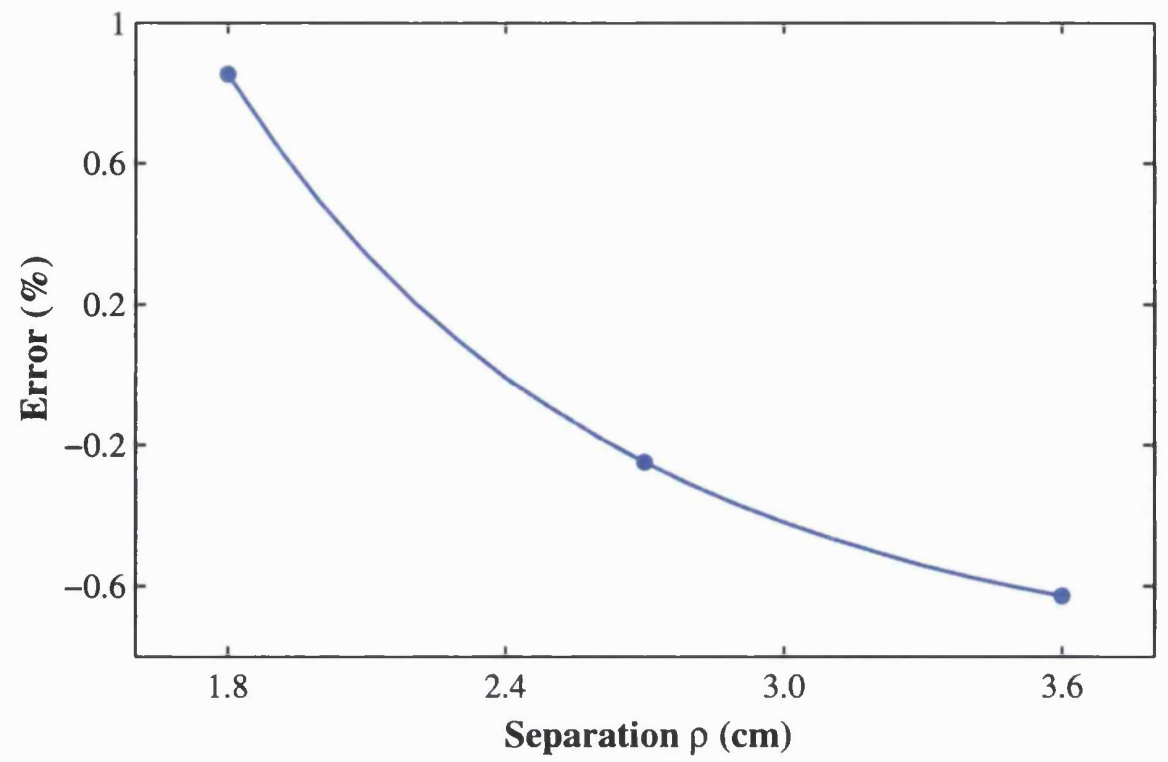

Figure 7.17: Percentage error of the linear approximation of spatially-resolved intensity compared to the exact diffusion theory solution as a function of source-detector separation in the range 1.8-3.6 cm (see text for details). The input intensity is $1, \mu_{a}$ and $\mu_{s}^{\prime}$ are 0.043 and $4.34 \mathrm{OD} \mathrm{cm}^{-1}$ respectively and the mid-point separation is $2.7 \mathrm{~cm}$. The markers indicate the errors at $1.8,2.7$ and $3.6 \mathrm{~cm}$.

and source-detector separations in the range $1.8-3.6 \mathrm{~cm}$, i.e. $\rho_{0}=2.7 \mathrm{~cm}$. Figure 7.17 shows that the percentage error from 1.8 to $3.6 \mathrm{~cm}$ is less than $1 \%$, indicating that Equation (7.17) is a good approximation of the spatially-resolved intensity given by the exact solution in the range of source-detector separations required for the penetration of light into deeper tissues such as muscle and brain.

The slope $a_{1}$ and offset $a_{0}$ of Equation (7.17) with respect to separation $\rho$ are given by

$$
\begin{aligned}
& a_{1}=-\frac{\mu_{\text {eff }} \rho}{2.3} \\
& a_{0}=\log _{10}\left(\frac{\mu_{e f f}+1 / \rho_{0}}{c \mu_{t}^{\prime}}\right)
\end{aligned}
$$

Thus, from the slope and offset given in Equation (7.18) one can obtain $\mu_{\text {eff }}$ and $\mu_{t}^{\prime}$, and hence $\mu_{a}$ and $\mu_{s}^{\prime}$, provided $c$ is known. However, the constant $c$ contains the incident intensity $I_{0}$, which as mentioned earlier, is difficult to measure independently. Instead the expression in Equation (7.17) can be referenced to a set of known (calibration) optical coefficients as follows

$$
\log _{10}\left(\frac{I_{a}\left(\rho, \rho_{0}\right)_{c a l}}{I_{a}\left(\rho, \rho_{0}\right)}\right)=\log _{10} \frac{\mu_{t}^{\prime}}{\mu_{t c a l}^{\prime}}+\log _{10}\left(\frac{\mu_{\text {eff cal }}+1 / \rho_{0}}{\mu_{\text {eff }}+1 / \rho_{0}}\right)+\frac{\mu_{\text {eff }}-\mu_{\text {eff cal }}}{2.3} \rho
$$

where $\mu_{\text {eff cal }}$ and $\mu_{t c a l}^{\prime}$ describe the calibration optical properties to which the measure- 
ments are referenced, and the unknown intensity $I_{0}$ cancels from the equation (as does the $\rho^{2}$ term on the left-hand side). Thus, it is the $\log$ ratio of the calibration intensity measurement to the sample intensity measurement that is compared to the expression in Equation (7.19), i.e. the difference attenuation with respect to the calibration measurement

$$
\begin{aligned}
\log _{10}\left(\frac{I_{\text {meas }}(\rho)_{\text {cal }}}{I_{\text {meas }}(\rho)}\right) & =A_{\text {meas }}(\rho)-A_{\text {meas }}(\rho)_{\text {cal }} \\
& =\Delta A_{\text {meas }}(\rho)
\end{aligned}
$$

The slope and offset of the expression in Equation (7.19) with respect to separation $\rho$ are now given by

$$
\begin{aligned}
& a_{1}=\frac{\mu_{e f f}-\mu_{e f f c a l}}{2.3} \\
& a_{0}=\log _{10} \frac{\mu_{t}^{\prime}}{\mu_{t c a l}^{\prime}}+\log _{10}\left(\frac{\mu_{e f f c a l}+1 / \rho_{0}}{\mu_{e f f}+1 / \rho_{0}}\right)
\end{aligned}
$$

Hence, absolute values of $\mu_{a}$ and $\mu_{s}^{\prime}$ can be determined from spatially-resolved intensity measurements, provided the optical properties of the calibration sample, $\mu_{a c a l}$ and $\mu_{s c a l}^{\prime}$, are known.

The ability of this method (hereafter known as the spatially-resolved approximation, SRA, method) to accurately recover changes in $\mu_{a}$ and $\mu_{s}^{\prime}$ was investigated using the Monte Carlo simulations (mentioned previously in this section) described in Section 7.2.1. The expression in Equation (7.20) was generated using the simulated reflectances at 1.8, 2.6 and $3.6 \mathrm{~cm}$, close to the source-detector separations at which the phantom measurements were made, with $\mu_{a c a l}$ and $\mu_{s c a l}^{\prime}$ equal to 0.087 and $4.34 \mathrm{OD} \mathrm{cm}^{-1}$ respectively, i.e. the values used to generate the reflectance in Simulation II described in Section 7.2.1 (see Table 7.5). (Note that the ratio of two reflectances is the same as the ratio of two intensities.) A linear least-squares regression against separation $\rho$ of the values thus obtained was performed, using the expressions in Equation (7.21) for the slope and offset to determine the optical coefficients. Table 7.7 shows the accuracy with which the SRA method can predict the optical coefficients from the reflectances of Simulations I, III and IV, assuming the correct estimates of $\mu_{a c a l}$ and $\mu_{s c a l}^{\prime}$. The errors range from 2 to $17 \%$, varying with the size of the changes in $\mu_{a}$ and $\mu_{s}^{\prime}$ from the calibration values. In general, the smaller the changes, the smaller the errors in the coefficients (Liu et al, 1995). However, it is evident that there is some correlation between the errors in the two coefficients, essentially because the least-squares regression attempts to minimise the error in the slope which contains $\mu_{\text {eff }}$, a combination of $\mu_{a}$ and $\mu_{s}^{\prime}$. Based on the findings of Chance et al (1995) mentioned 


\begin{tabular}{lllllll}
\hline Simulation & $\begin{array}{l}\mu_{a} \\
\left(\mathrm{OD} \mathrm{cm}^{-1}\right)\end{array}$ & $\begin{array}{l}\mu_{s}^{\prime} \\
\left(\mathrm{OD} \mathrm{cm}^{-1}\right)\end{array}$ & $\begin{array}{l}\mu_{a \text { pred }} \\
\left(\mathrm{OD} \mathrm{cm}^{-1}\right)\end{array}$ & $\begin{array}{l}\text { Error } \\
(\%)\end{array}$ & $\begin{array}{l}\mu_{s \text { pred }}^{\prime} \\
\left(\mathrm{OD} \mathrm{cm}^{-1}\right)\end{array}$ & $\begin{array}{l}\text { Error } \\
(\%)\end{array}$ \\
\hline I & 0.043 & 4.34 & 0.046 & 6.5 & 4.25 & -2.1 \\
III & 0.043 & 6.51 & 0.050 & 15.9 & 5.89 & -9.6 \\
IV & 0.087 & 6.51 & 0.102 & 17.4 & 5.65 & -13.3 \\
\hline
\end{tabular}

Table 7.7: Prediction of the optical coefficients used to generate Monte Carlo simulations of spatially-resolved reflectance by the SRA method, as a function of source-detector separation.

in Section 6.3.3, the transport scattering coefficient of the tissue phantom is expected to increase by approximately $1.3 \%$ (at $850 \mathrm{~nm}$ ) over the measured temperature range, much smaller than the $50 \%$ changes in $\mu_{s}^{\prime}$ for the Monte Carlo simulations. The temperatureinduced changes in $\mu_{a}$ of the phantom were estimated to be in the region of $0-1.4 \%$ over the whole wavelength range. Therefore, the SRA method should return reasonable estimates for the small changes in the optical properties of the tissue phantom, provided $\mu_{a c a l}$ and $\mu_{s c a l}^{\prime}$ are well estimated.

\subsubsection{Application to Tissue Phantom Data}

The approximation of the spatially-resolved intensity given by Equation (7.19) was used to obtain estimates of the optical properties of the tissue phantom, measured as a function of decreasing temperature (Section 6.3), by applying the SRA method described in Section 7.3.1. The calibration values $\mu_{a c a l}$ and $\mu_{s c a l}^{\prime}$, to which the changes with temperature are referenced, were the estimated $\mu_{a}$ and $\mu_{s}^{\prime}$ spectra of the tissue phantom at $37^{\circ} \mathrm{C}$ displayed in Figure 6.10 (see Section 6.3.3). Using the mid-point temperature $\left(37^{\circ} \mathrm{C}\right)$ of the measured range $\left(41-34^{\circ} \mathrm{C}\right)$ at which to reference the changes should produce smaller errors in the estimated optical coefficients than if the upper or lower temperature limit were chosen.

Figure 7.18 shows the reconstructed $\mu_{a}$ and $\mu_{s}^{\prime}$ spectra of the tissue phantom in the range $650-925 \mathrm{~nm}$ at $40.8,37.0$ and $33.8^{\circ} \mathrm{C}$. As expected, $\mu_{a}$ decreases with temperature, due to the decrease in the water absorption bands, whilst $\mu_{s}^{\prime}$ increases as the relative refractive index of the lipid droplets increases (see Section 6.3.3). The cross-talk (correlation) between $\mu_{a}$ and $\mu_{s}^{\prime}$ is evident in the slight ripples in the $\mu_{s}^{\prime}$ spectra at the positions of the water absorption bands. The changes in the optical coefficients are much larger than expected, however, approximately -12 to $-16 \%$ in $\mu_{a}$ and 8 to $16 \%$ in $\mu_{s}^{\prime}$ with decreasing temperature over the whole range. Using the same analytical technique Chance et 

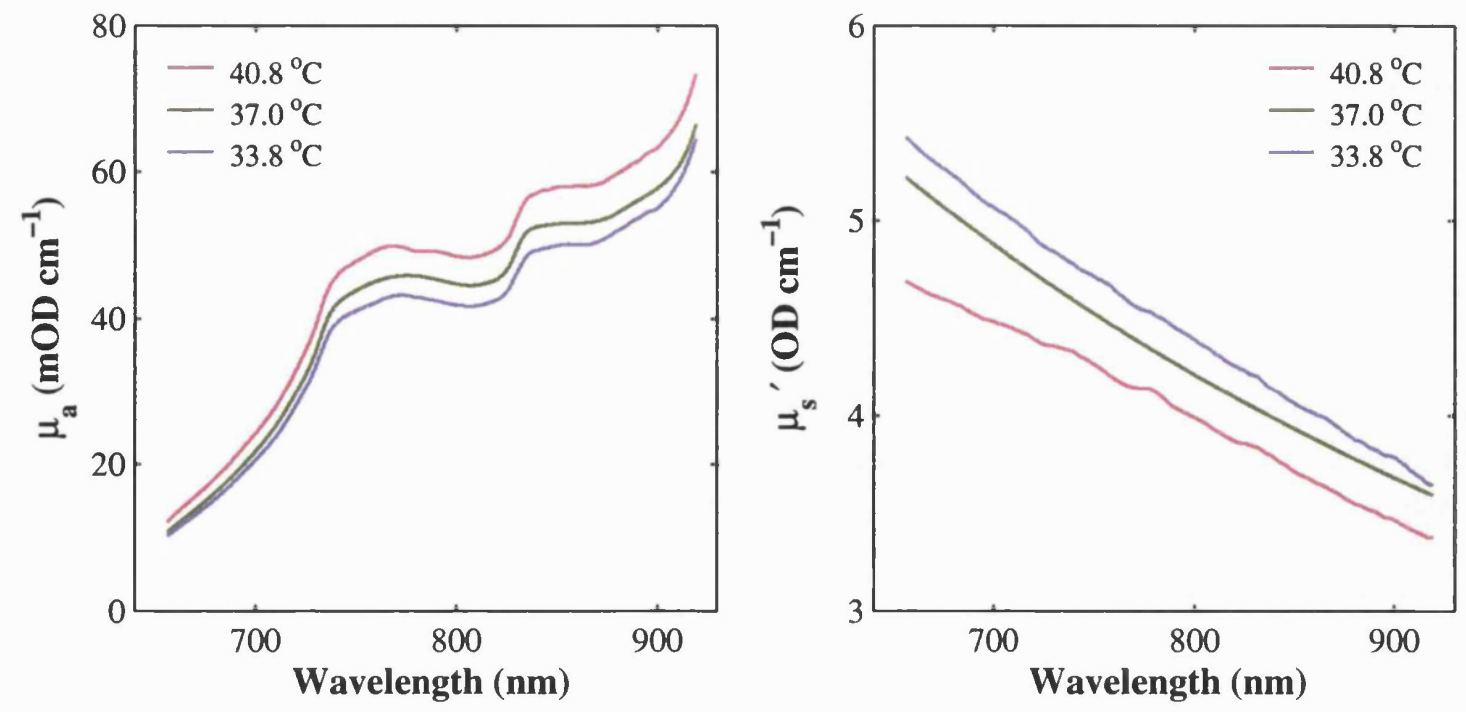

Figure 7.18: Absorption (left) and transport scattering (right) coefficients of the tissue phantom in the range $650-925 \mathrm{~nm}$ reconstructed using the SRA method, at temperatures of $40.8,37.0$ and $33.8^{\circ} \mathrm{C}$.

al (1995) studied the effects of temperature on the slope and offset of the approximation given in Equation (7.20) determined by spatially-resolved measurements of a $1 \%$ Intralipid solution (diluted with distilled water) in the range $20-38^{\circ} \mathrm{C}$. They observed temperature coefficients for the slope and offset of $-4.6 \times 10^{-4}$ and $-3.9 \times 10^{-4} \mathrm{OD} \mathrm{cm}^{-1}{ }^{\circ} \mathrm{C}^{-1}$ respectively at $850 \mathrm{~nm}$. As discussed previously in Section 6.3.3, this corresponded to a change in $\mu_{s}^{\prime}$ of $-0.18 \%{ }^{\circ} \mathrm{C}^{-1}$, with little change in $\mu_{a}$. In the current study, temperature coefficients of 3.4 and $-5.4 \times 10^{-3} \mathrm{OD} \mathrm{cm}^{-1}{ }^{\circ} \mathrm{C}^{-1}$ were observed for the slope and offset respectively at 850 $\mathrm{nm}$, i.e. the slope decreased and the offset increased with decreasing temperature, as shown in Figure 7.19. These ten-fold increases in the slope and offset indicate greater changes in the optical coefficients compared to the results of Chance et al (1995). Furthermore, the changes in the slope and offset suggest that whilst an increase in $\mu_{s}^{\prime}$ dominates the change in $\mu_{t}^{\prime}$, the decrease in $\mu_{a}$ is sufficient to produce a decrease in $\mu_{e f f}$ with decreasing temperature.

The fluctuations in the slope and offset at approximately $36-37^{\circ} \mathrm{C}$ and $39^{\circ} \mathrm{C}$ are also apparent in the reconstructed $\mu_{a}$ and $\mu_{s}^{\prime}$, plotted as a function of temperature at 850 $\mathrm{nm}$ in Figure 7.20, and on closer inspection appear to correlate with sharp (albeit small) 'jumps' in the attenuation measured at the smallest source-detector separation $(1.8 \mathrm{~cm})$. It is not clear what caused these attenuation drifts, although the fact that they do not appear in the measurements at 2.7 and $3.6 \mathrm{~cm}$ suggests some physical effect associated with (or in the vicinity of) the first detecting fibre, rather than a drift in the light source. 

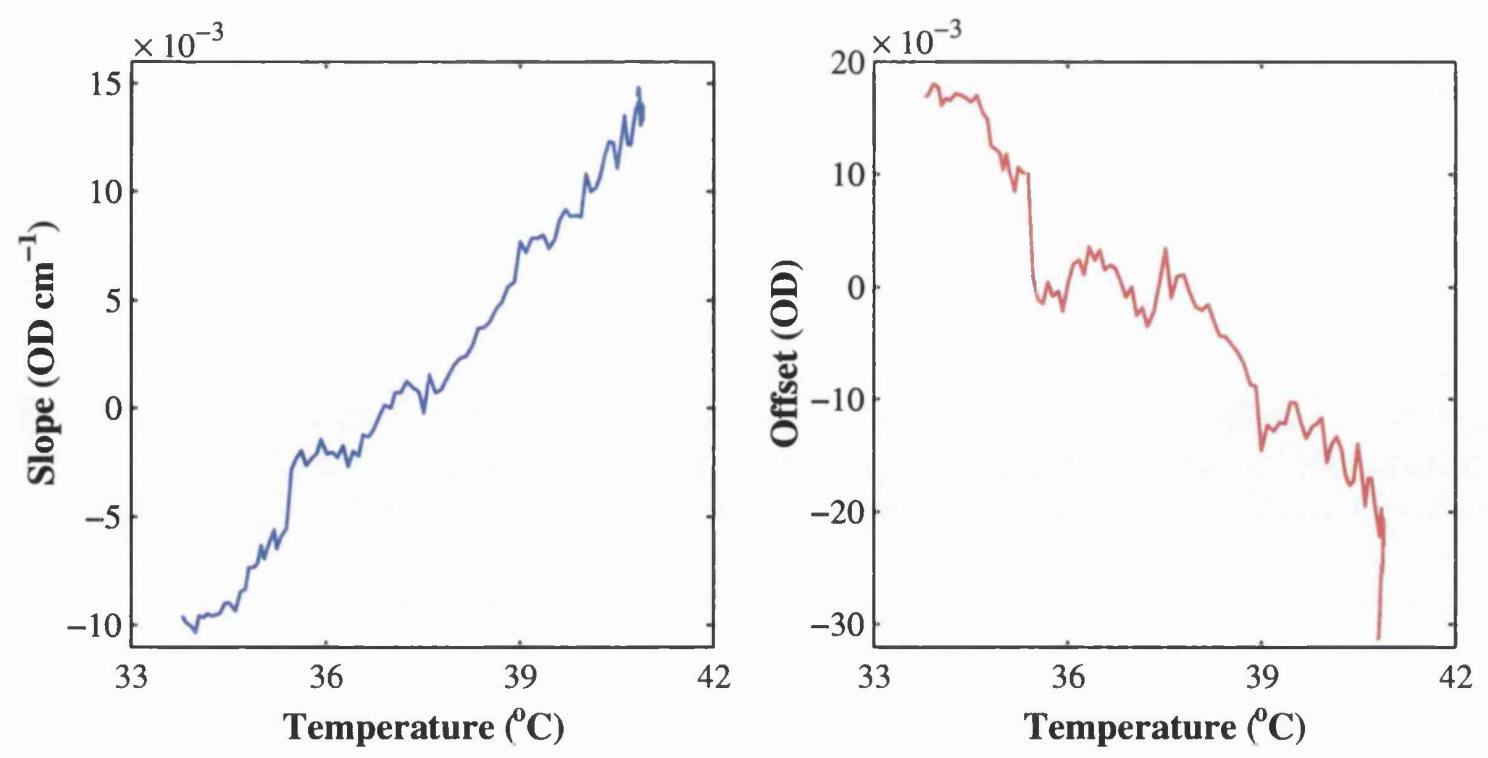

Figure 7.19: Effect of phantom temperature in the range $40.8-33.8^{\circ} \mathrm{C}$ on the slope (left) and offset (right) of the SRA approximation at $850 \mathrm{~nm}$ (see text for details).

Another interesting feature of the reconstructed optical coefficients is the large increase in $\mu_{s}^{\prime}$ and decrease in $\mu_{a}$ at an initial temperature of approximately $40.8^{\circ} \mathrm{C}$. In the first $15-20$ minutes of cooling the phantom temperature did not alter much, due to the significant thermal mass of the liquid. During this time, however, the attenuation measured at all three source-detector separations increased considerably ( 10 mOD). Because of their magnitude, it is unlikely that these changes are due to instrumental drifts, and hence must be attributed to changes in the optical properties of the phantom in the volume(s) probed. Although it is possible that the temperature sensors are not accurately reflecting the temperature of the interrogated liquid, this also seems unlikely, based on their close proximity to the fibres and the good agreement between them (indicating an equilibration of temperature in the surrounding region). The most probable explanation for this initial change in attenuation is a time-dependent 'stabilisation' of the liquid phantom in terms of its flow and density around the fibres. With regard to the optical properties, therefore, this attenuation change is likely to be governed by an increase in $\mu_{s}^{\prime}$, rather than $\mu_{a}$.

As a further investigation, the absorption spectra of saline, soybean oil and copper sulphate were fitted to the attenuation spectra measured at the smallest source-detector separation using the second derivative method described in Section 7.1. The regression coefficients of the second derivative fit are proportional to the concentration of each component (see Section 7.1.3). For the soybean oil, the lipid component of Intralipid, the regression coefficient did not vary significantly over the measured temperature range. The regression coefficients for saline and copper sulphate are shown in Figure 7.21. The saline 

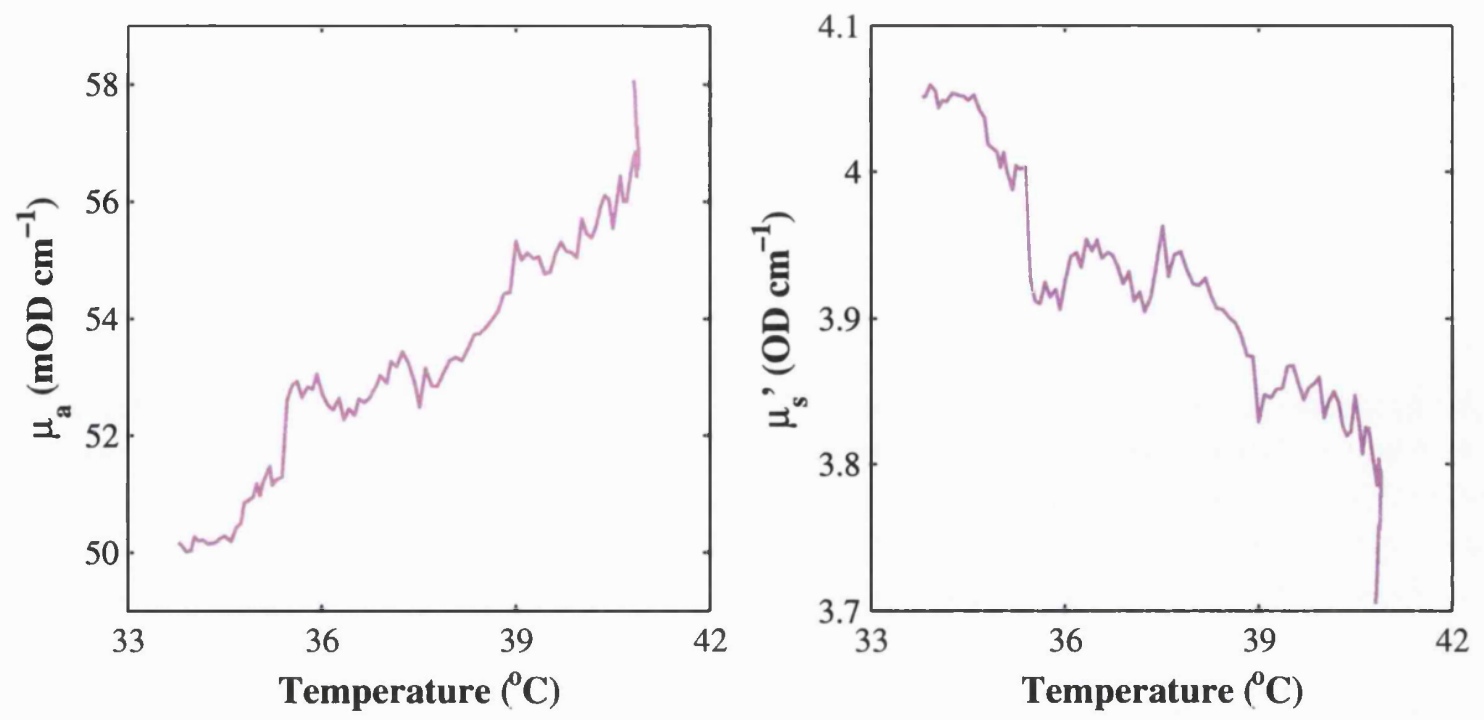

Figure 7.20: Reconstructed absorption (left) and transport scattering (right) coefficients of the tissue phantom at $850 \mathrm{~nm}$ as a function of temperature (see text for details).

component decreases linearly with decreasing temperature, indicating a decrease in saline concentration and/or temperature (the mean spectrum from the saline data set was fitted). The $\mathrm{CuSO}_{4}$ component, however, decreases significantly at an initial temperature of approximately $41^{\circ} \mathrm{C}$, the phenomenon also seen in the reconstruction of $\mu_{a}$ by the SRA method, displayed in Figure 7.20. As the ratio of the regression coefficients represents the ratio of the component concentrations, it is apparent that the concentration of $\mathrm{CuSO}_{4}$ decreases relative to that of saline at a temperature of $41^{\circ} \mathrm{C}$. This decrease in the $\mathrm{CuSO}_{4}$ component could reflect a true decrease in the contribution to absorption by $\mathrm{CuSO}_{4}$ during the equilibration period discussed above, or it could be an artifact of the fitting. As discussed in Section 7.1.1, the SDS method approximates a linear relationship between attenuation and absorption in the second derivative, ignoring contributions from scatter. If $\mu_{s}^{\prime}$ has an appreciable second derivative, the first and second order $\mu_{s}^{\prime}$ terms defined in the SDS approximation of Equation (7.1) may be significant. This would result in an underestimation of the chromophore contributions, which would worsen as $\mu_{s}^{\prime}$ increased, and may be more significant in chromophores present at lower concentrations. In the SRA method, changes in the reconstructed optical coefficients are also correlated. As $\mu_{s}^{\prime}$ increases, the predicted $\mu_{s}^{\prime}$ will be increasingly overestimated, whilst $\mu_{a}$ will be underestimated, as demonstrated by the MC simulations in Table 7.7. This could explain the large decrease in the reconstructed $\mu_{a}$ of the phantom on cooling from $41{ }^{\circ} \mathrm{C}$, due to the significant increase in scattering. However, since the saline component seems relatively unaffected by the increase in scattering, as demonstrated by the second derivative fit in 

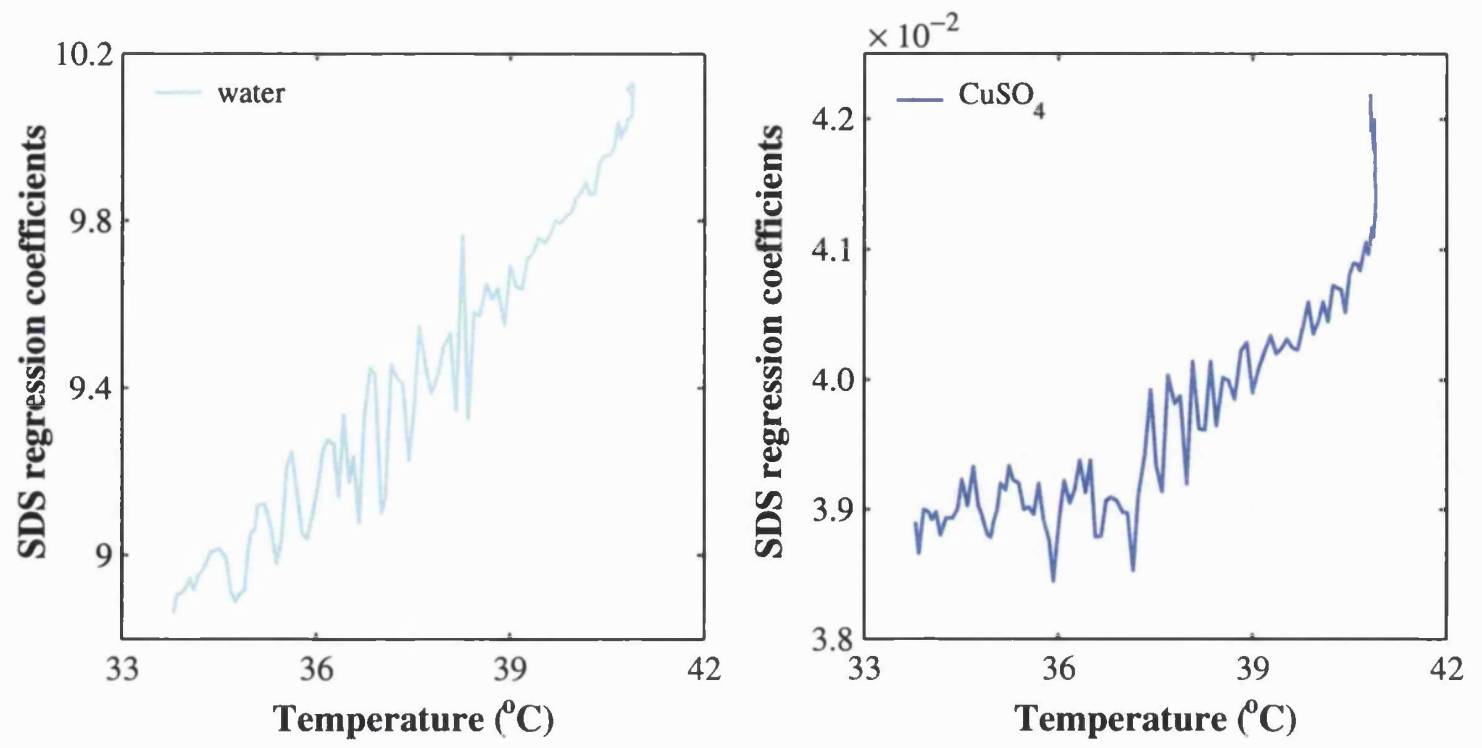

Figure 7.21: Second derivative regression coefficients of saline (left) and copper sulphate (right) components as a function of temperature for a source-detector separation of 1.8 $\mathrm{cm}$. Note the large decrease in the copper sulphate component at an initial temperature of $\sim 41^{\circ} \mathrm{C}$ (see text for details).

Figure 7.21, temperature prediction based on the change in absorption by saline may still be possible in the presence of a large increase in $\mu_{s}^{\prime}$. This will be investigated in the following section.

\subsubsection{Temperature Prediction}

The temperature of the tissue phantom was then predicted using the PLSR calibration described in Section 5.3.6. The calibration was repeated for the saline measurements as a function of temperature (Section 5.2.2), since although not considerably different to the measurements of the water absorption spectra (see Section 5.2.2.2 for a comparison), small differences may affect the construction of the PLSR weight and loading vectors. The linear fit to the reconstructed phantom absorption spectra included the PLSR weight and loading vectors of the saline calibration, the mean absorption spectrum of the saline calibration data set, the soybean oil spectrum and the $\mathrm{CuSO}_{4}$ absorption spectrum. Although the phantom contained $9 \%$ pure water as well as $89.7 \%$ saline, the mean water spectrum was not included in the fit because of its similarity to the mean saline spectrum.

Figure 7.22 shows the measured against predicted temperature of the tissue phantom using the first PLSR factor. As temperature alters from the mid-point of $37^{\circ} \mathrm{C}$, it can be seen that the changes in temperature predicted by the PLSR scores are underestimated in both directions. The large change in the reconstructed $\mu_{a}$ at approximately $41^{\circ} \mathrm{C}$ 


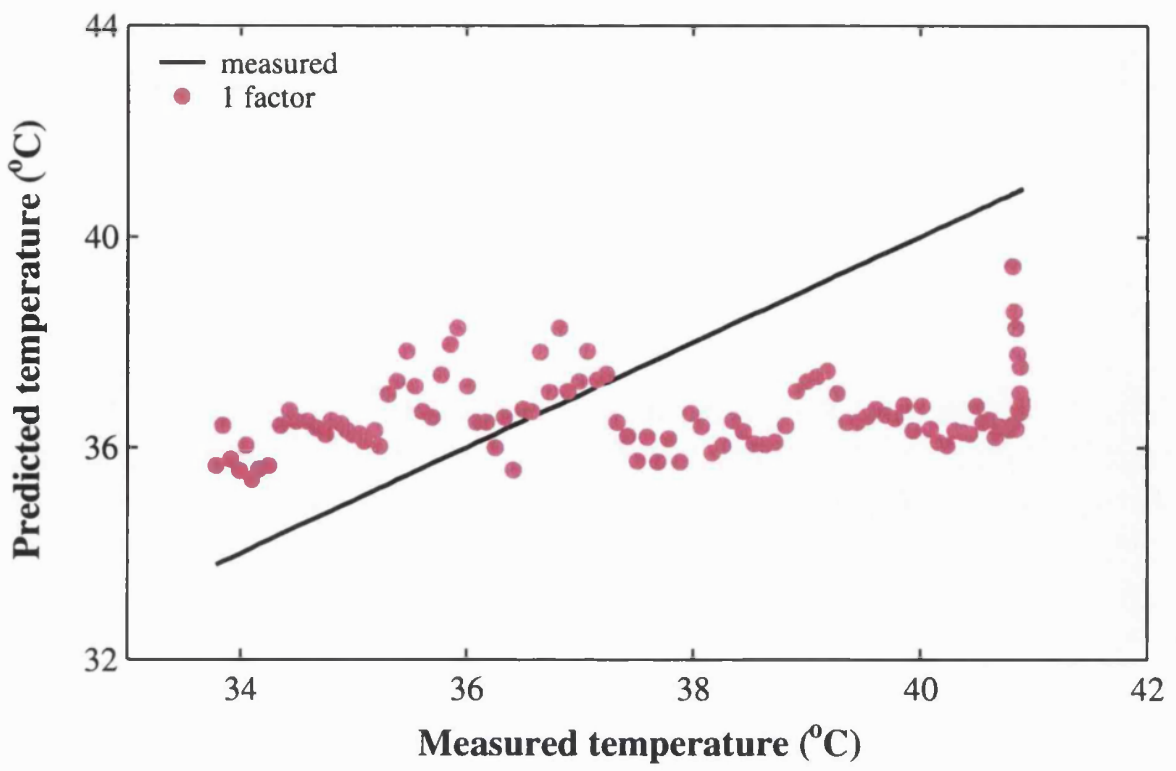

Figure 7.22: Tissue phantom temperature predicted from reconstructions of $\mu_{a}$ using the SRA method for the inclusion of one PLSR calibration factor (see text for details).

(see Figure 7.20), manifests itself as an increase in the predicted temperature at $41^{\circ} \mathrm{C}$. Including further factors in the fit worsened the temperature prediction. The accurate prediction of temperature relies on the accurate determination of the scores of the factors included and the concentration of the mean spectrum of the saline data set, i.e. the actual saline concentration. Since the scores are normalised to the saline concentration (see Appendix C), the underestimation in temperature changes from the mid-point could be due to either an underestimation of changes in the scores or an overestimation of changes in the saline concentration. Inspection of the linear fitting coefficients determined that the saline concentration varied from $98.6 \%$ at $37^{\circ} \mathrm{C}$ (indicating the combined concentration of the saline and water of $98.7 \%$ in the reference spectrum) to approximately $108 \%$ at $41^{\circ} \mathrm{C}$ and $95 \%$ at $34^{\circ} \mathrm{C}$. Clearly the saline concentration should not exceed $100 \%$, suggesting that the changes in concentration are erroneously overestimated. However, if the scores are not normalised to the saline concentration, the predicted temperature is virtually unchanged, which suggests that the scores themselves are underestimated. This could arise due to either cross-talk with the other components, the copper sulphate and the soybean oil, or due to an underestimation of the reconstructed $\mu_{a}$ changes. Altering the scale of the $\mu_{a c a l}$ and $\mu_{s c a l}^{\prime}$ spectra used as the reference optical coefficients for the SRA reconstruction from 0.1 to 5 did not significantly alter the temperature prediction, other than a slight scaling in the values. Furthermore, altering the component concentrations in the estimated $\mu_{a \text { cal }}$ spectrum at $37^{\circ} \mathrm{C}$ did not change the 'shape' of the predicted versus 
measured temperature relationship, only the scale or offset of the values.

It seems apparent, therefore, that the scores are underestimated because the changes in either $\mu_{a}$ or $\mu_{s}^{\prime}$, or both, are large enough to induce significant errors in their estimation by the SRA method. As indicated in Table 7.7, as $\mu_{a}$ decreases and $\mu_{s}$ increases (Simulation III), analogous to a decrease in temperature, the absorption coefficient returned will be overestimated, i.e. the change in $\mu_{a}$ will be underestimated. Correspondingly, as $\mu_{a}$ increases and $\mu_{s}^{\prime}$ decreases (with increasing temperature), $\mu_{a}$ will be underestimated, which also underestimates the changes in $\mu_{a}$. A significant attenuation increase was seen in the first 15-20 minutes of the experiment that was not temperature-related and was reconstructed as an increase in $\mu_{s}^{\prime}$, and a corresponding decrease in $\mu_{a}$. The effect of this change in $\mu_{s}^{\prime}$, almost a third of the total change in $\mu_{s}^{\prime}$ in the measured temperature range, could be to underestimate all subsequent changes in $\mu_{a}$. These results will be discussed again in Chapter 8. 


\section{Chapter 8}

\section{Conclusions}

This chapter summarises the work carried out for this project at each stage of its development. Suggestions for subsequent work are also discussed.

Section 8.1 discusses the main findings of the work performed in the following three areas: measurement of the temperature-dependence of the water absorption spectrum, calibration of the water spectrum against temperature and the prediction of temperature from NIRS measurements of tissue or a tissue-like phantom. In Section 8.2 the possibilities for further work are detailed, with attention to the clinical application of this method, which is the ultimate goal. 


\subsection{Project Summary}

\subsubsection{Temperature-Dependence of the NIR Water Spectrum}

In Section 5.1 the measurement of the NIR water absorption spectrum as a function of temperature in the range $44-28^{\circ} \mathrm{C}$ was described. As discussed in this section, the implementation of these measurements was not as straight-forward as conventional absorption spectroscopy. Hence, the experimental design required careful consideration and the development of a methodology to obtain an accurate representation of the temperature response of the water spectrum. A detailed description of the spectroscopic analysis involved in the determination of the absorption coefficient from the measurements was also given. The temperature coefficient of the water absorption bands positioned at approximately 740,840 and $970 \mathrm{~nm}$ was found to be in the range $0.5-0.8 \%{ }^{\circ} \mathrm{C}^{-1}$. Although the $970 \mathrm{~nm}$ absorption band exhibited the largest temperature coefficient, the measurement of this band in tissue with the current system is difficult due to the poor SNR, hence the calibration and temperature prediction algorithms were based on the measurements in the range $650-920 \mathrm{~nm}$, where the attenuation in tissue is considerably lower (see also Section 8.2 for further comments on this point). Although the solution of polar compounds may disrupt hydrogen-bonding within bulk water, measurements of the temperature-dependence of an organic cellulose-based compound and isotonic saline were not significantly different from those of pure water.

One particular problem associated with the current instrumental system is its inability to account for drift in the light source output. Although, as described in Section 5.1.3, the potential for this drift was minimised by the experimental design, it can in fact contribute an unknown amount to changes in the measured intensity assumed to be due to temperature-dependent changes in water absorption. The extent of the drift could be monitored by using a 'double-beam' arrangement, in which the incident beam is split by a beam-splitter and one part of the beam passes through the sample, the other through a reference, e.g. air. By use of a mechanical chopper the light from the two beams can be alternately sampled by the detector. However, whilst this may give an indication of the magnitude of the signal drift, it could not be used to correct for it unless the reference was identical to the one in the water absorption measurement, i.e. one would need a duplicate set-up to that described in Section 5.1.1 in which to make a reference measurement. 


\subsubsection{Calibration of the Water Spectrum Against Temperature}

Section 5.3 described the calibration of the measurements of the water absorption spectrum against temperature. A variety of calibration techniques were investigated, including PCR and PLSR, both of which attempt to describe the water absorption spectrum as a combination of components, known as principal components or factors, that vary linearly with temperature. It was shown that the PLSR calibration is superior to the PCR calibration in that it relates the derived factors more closely to water temperature in the calibration step than does PCR. This was demonstrated by the ability of the PLSR calibration to yield an optimal number of factors for the model by determination of a minimum SEP using the cross-validation method. In the PCR calibration the SEP decreased with increasing number of PCs, indicating an overfitting of the spectrum with smaller and smaller PCs which introduce noise into the system. For the inclusion of 3 PCs or factors, the PCR and PLSR calibrations yielded similar values for the SEP of approximately $0.035^{\circ} \mathrm{C}$.

\subsubsection{Temperature Prediction Algorithms}

Chapter 7 discussed the algorithms developed to predict temperature from the temperature-resolved NIRS measurements described in Chapter 6. Algorithm I used second derivative spectroscopy to approximate a linear relationship between attenuation and absorption, hence allowing direct regression of the PCR loading vectors on to the in vivo attenuation spectra. Using the water absorption data, the SDS method was capable of predicting temperature to within $0.4^{\circ} \mathrm{C}$, the poorer prediction relating in part to the noise inflation in the second derivative. In simulations of tissue attenuation, the predicted temperature was overestimated by approximately $7-8^{\circ} \mathrm{C}$, due to cross-talk between the chromophore spectra and the loading vectors and noise inflation.

Algorithm II employed a non-linear optimisation technique to reconstruct absorption differences, relative to the initial measurement, from the in vivo study of the human forearm, assuming the transport scattering coefficient to be fixed with temperature. The initial underlying absorption spectrum was determined by fitting to the first 50 spectra, during which the tissue temperature did not change, using SDS. Temperature was predicted by once again fitting with the PCR loading vectors. The standard error of prediction was approximately $1.2^{\circ} \mathrm{C}$. By inspection of the temperature residuals a significant change in the temperature prediction was seen to occur at around $34^{\circ} \mathrm{C}$. Simulating a linear change in the transport scattering coefficient with temperature did not remove this non-linearity. 
It was noted that this effect may be due to a decrease in blood flow or tissue perfusion with decreasing temperature. The temperature-dependence of the haemoglobin spectra was discussed in Section 2.1.2, although this was not taken into account in Algorithm II. If the $\mathrm{Hb}$ and $\mathrm{HbO}_{2}$ spectra were measured as a function of temperature, a PCA analysis of the two chromophore spectra as a function of temperature could be performed. The loading vectors for $\mathrm{Hb}$ and $\mathrm{HbO}_{2}$ could then be included in the fit to the reconstructed absorption spectra, as with water.

Algorithm III made use of the spatially-resolved measurements of the tissue-like phantom. A linear approximation of the diffusion theory solution for spatially-resolved diffuse reflectance from a semi-infinite medium was fitted to the experimental data obtained at three source-detector separations, to determine changes in both absorption and transport scattering coefficients relative to an assumed set of coefficients at $37^{\circ} \mathrm{C}$. The changes in temperature from the reference point of $37^{\circ} \mathrm{C}$ were systematically underestimated by this method. An initial increase in attenuation (possibly due to settling or 'stabilisation' of the phantom) of the phantom at a temperature of approximately $41^{\circ} \mathrm{C}$ resulted in a corresponding increase in the transport scattering coefficient and a decrease in the absorption coefficient. It was suggested that this was the cause of the underestimation of temperature, due to the subsequent underestimation of changes in absorption following a large temperature-independent increase in scattering. These results could almost certainly be improved by optimising the experimental design, as will be discussed in Section 8.2.

\subsection{Further Work}

Possibly one of the most effective ways of reducing many of the problems associated with monitoring tissue temperature by NIRS would be to use the changes in the $970 \mathrm{~nm}$ water absorption band, i.e. monitor spectral changes in the range $900-1050 \mathrm{~nm}$, say, as opposed to 650-900 nm. Provided sufficient depth penetration can be achieved at these wavelengths, three improvements over the current method can be identified immediately:

i) an increase in the percentage changes in water absorption with temperature at 970 $\mathrm{nm}$, i.e. a greater sensitivity of the technique to resolve changes in temperature

ii) a decrease in tissue scattering at longer wavelengths (Matcher et al, 1997)

iii) lower absorption by $\mathrm{Hb}$ and therefore less potential for interference between the temperature-dependence of its absorption spectrum and that of water. 
To implement this method, however, one would need an entirely different detector, since the silicon-based CCD detector used for these measurements rapidly loses sensitivity beyond $900 \mathrm{~nm}$. A suitable detector might be an indium gallium arsenide (InGaAs) photodiode array, which is sensitive from around 800 to $1800 \mathrm{~nm}$ (or $2500 \mathrm{~nm}$ with impurity doping). The obvious disadvantage of this would be the loss of the NIR range from 650$800 \mathrm{~nm}$ for simultaneously monitoring cerebral oxygenation, although the feasibility of using the 900-1050 $\mathrm{nm}$ range for the latter has not been investigated.

In the spatially-resolved measurements of the tissue phantom it was noted that the measurement at the first source-detector separation was affected by some unknown disturbance, causing sharp 'jumps' in the signal that did not appear in the other measurements. These jumps could be observed in both the absorption and transport scattering coefficients determined by the SRA method. Such perturbations could be reduced by increasing the number of detecting fibres employed, which will improve the linear regression of the approximated expression to the experimental data and thus reduce the sensitivity of the reconstructed optical coefficients to potential 'outliers'. The range of source-detector separations used will be limited by the SNR and the dynamic range of the detector. Furthermore, one must consider the range of penetration depths interrogated by the light at each source-detector separation and the heterogeneity of the tissue. An ideal probe should contain many detecting fibres positioned in close proximity to one another within a specified distance, suitable for the interrogating the tissue of interest whilst keeping the range of the light penetration as narrow as possible to reduce the effects of tissue heterogeneity. A trade-off will thus exist between SNR and the approximation of tissue homogeneity by using many small diameter fibres.

In terms of clinical measurements of brain temperature in hypothermic infants, the practical implementation of the proposed method should be relatively trouble-free, or at least have no additional problems than previously established methods of NIRS monitoring in new-born infants. The optical fibres can be positioned appropriately on the infant's forehead without affecting the cooling cap (see Figure 1.2) or the care of the infant. A greater concern is the potential for heating of the skin surface due to the continuous input of light, particularly if the large $970 \mathrm{~nm}$ absorption band were employed, which could affect the local temperature distribution within the brain. This should be minimised at all costs by the use of appropriate heat filters.

With regards to the accurate measurement of temperature within the brain using NIRS, an important issue is the extent of the temperature gradient that will exist between 


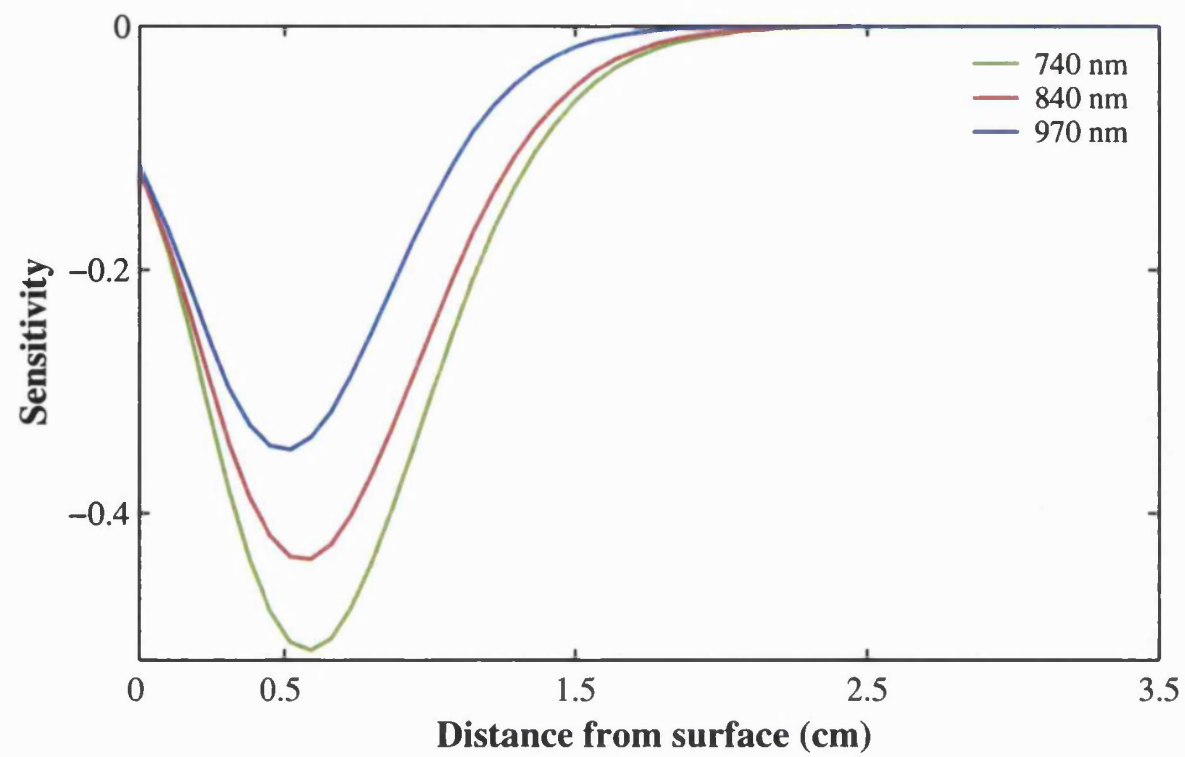

Figure 8.1: Cross-section of a PMDF calculated for absorption in a 2D model of the neonatal head as a function of depth into the head from the surface (see text for details)

the cooled surface and the core. This point is relevant to the proposed method because, as discussed earlier, the light samples a volume of tissue distributed over a certain depth, hence may also be sampling a temperature gradient within this volume. Modelling of the temperature distribution within the brain during cooling will be essential to determine the range of penetration depths within which a meaningful average temperature can be determined ( $\mathrm{Xu}$ et al, 1999; Zhu and Diao, 2001). Since the average photon depth depends on the optical coefficients, the light at different wavelengths will penetrate to different depths within the brain, hence passing through tissue at different temperatures. Provided the depth resolution is great enough, based on the extent of the temperature gradient, it may be possible to determine brain temperature as a function of depth into the brain using NIRS. Figure 8.1 shows the cross-section of a photon-measurement density function (PMDF) at three wavelengths for a 2-D, homogeneous model of the infant head with a circular diameter of $7 \mathrm{~cm}$. The PMDF, calculated by the finite element method (Schweiger et al, 1995), represents the sensitivity of the measurement to a local change in the optical properties of a medium, i.e. the region sampled by the light. The PMDFs of Figure 8.1 were calculated for absorption with the cross-sectional axis bi-secting the source and detector, separated by an arc of $3 \mathrm{~cm}$ in the simulation. The minima represent the maximum sensitivity in the cross-section. The optical coefficients are the mean for white and grey matter of the neonatal brain, taken from the data of van der Zee (1993). As the absorption coefficient increases with wavelength, the sensitivity decreases, i.e. less photons are detected, as shown in Figure 8.1. The scattering coefficient also decreases 
with increasing wavelength, which causes the penetration depth of the photons to decrease, as shown by a shift in the minima of the PMDFs. This brief study shows that it may be possible to determine brain temperature at different depths by making use of the wavelength-dependence of light penetration into tissue, but further investigation would be required. 


\section{Appendix A}

\section{Error Analysis for Calculation of $\mu_{a \mathbf{T}}$}

This appendix describes the analysis performed to determine the uncertainty in the calculation of the water absorption coefficient as a function of temperature, based on the errors in the intensity and height measurements (errors in the temperature measurements have not been included). Assuming $f(x, y)$ to be a function of $x$ and $y$ and the errors on $x$ and $y(\triangle x$ and $\triangle y)$ to be independent of one another, the error (squared) on $f$ is given by

$$
(\triangle f(x, y))^{2}=\left(\frac{\partial f}{\partial x}\right)^{2}(\Delta x)^{2}+\left(\frac{\partial f}{\partial y}\right)^{2}(\Delta y)^{2}
$$

The general formulation, on which the error calculations of this section are based, is then given by

$$
\Delta f(a)=\sqrt{\sum_{i}^{k}\left(\frac{\partial f}{\partial a_{i}}\right)^{2}\left(\Delta a_{i}\right)^{2}}
$$

where $\boldsymbol{a}$ contains the $k$ independent variables $a_{i}$. For the purpose of this analysis the errors in the intensity and height measurements are assumed to be independent of one another.

The error on an intensity measurement $I$ is dominated by the shot noise, as discussed in Section 3.2, which is given by

$$
\triangle I=\sqrt{I}
$$

In fact, the noise on a single intensity measurement is some fraction of this equal to $\alpha \triangle I$, such that the mean of many observations of $I$ has noise equal to $\Delta I$, i.e. $\alpha$ is normally distributed around 1. For this analysis, however, it has been assumed that the error on each intensity measurement is $\sqrt{I}$, which is an approximation. The error on the (base 10) 
logarithm of the intensity, determined by Equation (A.2), is then given by

$$
\triangle \log _{10} I=\frac{1}{2.3 \sqrt{I}}
$$

As discussed in Section 5.1.2.1, in order to reduce this error the acquisition software averages over the $\log _{10}$ of $n$ spectra (values of $n$ given in Tables 5.1 and 5.2) such that each intensity spectrum stored is given by

$$
\log _{10} I_{\text {avg }(n)}=\frac{1}{n} \sum_{i}^{n} \log _{10} I_{i}
$$

The error on the averaged $\log _{10}$ spectrum is given by

$$
\triangle \log _{10} I_{\text {avg }(n)}=\frac{1}{2.3 n} \sqrt{\sum_{i}^{n} \frac{1}{I_{i}}}
$$

Assuming that each intensity measurement $I_{i}$ will be distributed around the mean we can approximate each $I_{i}$ as $I_{\text {avg }(n)}$, in which case the error on the averaged $\log _{10}$ spectrum can be given by

$$
\triangle \log _{10} I_{\text {avg }(n)}=\frac{1}{2.3 \sqrt{n I_{\text {avg }(n)}}}
$$

The $\left(\log _{10}\right)$ spectra were further averaged over $m$ spectra on exportation from the acquisition software such that the maximum temperature change between spectra was $0.05^{\circ} \mathrm{C}$. The doubly-averaged intensity measurement is then given by

$$
\log _{10} I_{\text {avg }(n, m)}=\frac{1}{m} \sum_{i}^{m} \log _{10} I_{\text {avg }(n) i}
$$

Making a similar approximation to that used in Equation (A.7) the error on the 'doublyaveraged' intensity measurement is given by

$$
\triangle \log _{10} I_{\text {avg }(n, m)}=\frac{1}{2.3 \sqrt{n m I_{\text {avg }(n, m)}}}
$$

Equations (A.7) and (A.9) will be used to determine the errors on the intensity measurements $\log _{10} I_{r \mathrm{~T}_{r}}, \log _{10} I_{f} \mathrm{~T}_{r}$ and $\log _{10} I_{f \mathrm{~T}}$ used to calculate $\mu_{a \mathrm{~T}}$ in Equations (5.21) and (5.22).

The error in the water height measurement will be dominated by the accuracy of the syringe driver to deliver a certain volume (the uncertainty in the diameter of the glass tube was deemed to be negligible in comparison so has not been included). This accuracy 
was reported to be $\pm 3 \%$ by the manufacturers of the syringe driver, hence the error on the height measurement is given by

$$
\triangle h=0.03 h
$$

In the calculation of $\mu_{a \mathrm{~T}}$ the concept of a standardising temperature was introduced to ensure that the absorption coefficient at any temperature $T$ is always expressed at the same water concentration, i.e. that at the standardising temperature $\mathrm{T}_{S}$. The height $h$ must therefore be adjusted to account for this, as given by Equation (5.11). The errors in the heights $h_{1} \mathrm{~T}_{S}$ and $h_{2} \mathrm{~T}_{S}$ used in the calculation of $\mu_{a \mathrm{~T}}$ in Equations (5.21) and (5.22) are given by

$$
\begin{aligned}
& \Delta h_{1 \mathrm{~T}_{S}}=0.03\left(\frac{d_{\mathrm{T}_{1}}}{d_{\mathrm{T}_{S}}}\right) h_{1 \mathrm{~T}_{1}} \\
& \Delta h_{2 \mathrm{~T}_{S}}=0.03\left(\frac{d_{\mathrm{T}_{2}}}{d_{\mathrm{T}_{S}}}\right) h_{2 \mathrm{~T}_{2}}
\end{aligned}
$$

where $d_{\mathrm{T}_{1}}, d_{\mathrm{T}_{2}}$ and $d_{\mathrm{T}_{S}}$ are the water densities defined in Section 5.1.2.2.

The following two sections will work through the error analysis for the absorption coefficient determined by both Methods I and II (Sections 5.1.2.1 and 5.1.2.3) based on the propagation of the errors in intensity and height measurements defined here. As described by Equation (A.2), the error in the absorption coefficient will be the sum of the contributions from the five independent variables $\log _{10} I_{r \mathrm{~T}_{r}}, \log _{10} I_{f} \mathrm{~T}_{r}, \log _{10} I_{f} \mathrm{~T}, h_{1 \mathrm{~T}_{S}}$ and $h_{2} \mathrm{~T}_{S}$ (or three of these in the case of Method II).

\section{A.1 Method I}

The expression for the absorption coefficient $\mu_{a} \mathrm{~T}$ calculated using Method I was given by Equation (5.21). This expression is differentiated with respect to each of the variables listed above to give the following:

$$
\begin{gathered}
\frac{\partial \mu_{a \mathrm{~T}}}{\partial \log _{10} I_{r \mathrm{~T}_{r}}}=\frac{1}{h_{2 \mathrm{~T}_{S}}} \\
\frac{\partial \mu_{a \mathrm{~T}}}{\partial \log _{10} I_{f \mathrm{~T}_{r}}}=-\frac{h_{1 \mathrm{~T}_{S}}}{h_{2 \mathrm{~T}_{S}}\left(h_{1 \mathrm{~T}_{S}}+h_{2 \mathrm{~T}_{S}}\right)} \\
\frac{\partial \mu_{a \mathrm{~T}}}{\partial \log _{10} I_{f \mathrm{~T}}}=-\frac{1}{h_{1 \mathrm{~T}_{S}}+h_{2 \mathrm{~T}_{S}}}
\end{gathered}
$$




$$
\begin{gathered}
\frac{\partial \mu_{a \mathrm{~T}}}{\partial h_{1 \mathrm{~T}_{S}}}=\frac{\log _{10} I_{f \mathrm{~T}}-\log _{10} I_{f} \mathrm{~T}_{r}}{\left(h_{1 \mathrm{~T}_{S}}+h_{2 \mathrm{~T}_{S}}\right)^{2}} \\
\frac{\partial \mu_{a \mathrm{~T}}}{\partial h_{2} \mathrm{~T}_{S}}=\frac{h_{2 \mathrm{~T}_{S}}^{2} \log _{10} I_{f \mathrm{~T}}+h_{1 \mathrm{~T}_{S}}\left(h_{1 \mathrm{~T}_{S}}+2 h_{2 \mathrm{~T}_{S}}\right) \log _{10} I_{f} \mathrm{~T}_{r}-\left(h_{1 \mathrm{~T}_{S}}+h_{2 \mathrm{~T}_{S}}\right)^{2} \log _{10} I_{r \mathrm{~T}_{r}}}{h_{2 \mathrm{~T}_{S}}^{2}\left(h_{1 \mathrm{~T}_{S}}+h_{2} \mathrm{~T}_{S}\right)^{2}}
\end{gathered}
$$

The error (squared) in the absorption coefficient is therefore given by

$$
\begin{gathered}
\left(\triangle \mu_{a \mathrm{~T}}\right)^{2}=\left(\frac{\partial \mu_{a \mathrm{~T}}}{\partial \log _{10} I_{r} \mathrm{~T}_{r}}\right)^{2}\left(\Delta \log _{10} I_{r} \mathrm{~T}_{r}\right)^{2}+\left(\frac{\partial \mu_{a \mathrm{~T}}}{\partial \log _{10} I_{f} \mathrm{~T}_{r}}\right)^{2}\left(\Delta \log _{10} I_{f} \mathrm{~T}_{r}\right)^{2} \\
+\left(\frac{\partial \mu_{a \mathrm{~T}}}{\partial \log _{10} I_{f \mathrm{~T}}}\right)^{2}\left(\Delta \log _{10} I_{f} \mathrm{~T}\right)^{2}+\left(\frac{\partial \mu_{a \mathrm{~T}}}{\partial h_{1 \mathrm{~T}_{S}}}\right)^{2}\left(\Delta h_{1 \mathrm{~T}_{S}}\right)^{2} \\
+\left(\frac{\partial \mu_{a \mathrm{~T}}}{\partial h_{2 \mathrm{~T}}}\right)^{2}\left(\Delta h_{2} \mathrm{~T}_{S}\right)^{2}
\end{gathered}
$$

where $\Delta h_{1 \mathrm{~T}_{S}}$ and $\Delta h_{2} \mathrm{~T}_{S}$ are given by Equation (A.11) and as follows from Equations (A.7) and (A.9):

$$
\begin{gathered}
\triangle \log _{10} I_{r \mathrm{~T}_{r}}=\frac{1}{2.3 \sqrt{n_{r} I_{r} \mathrm{~T}_{r}}} \\
\triangle \log _{10} I_{f} \mathrm{~T}_{r}=\frac{1}{2.3 \sqrt{n_{f} m_{f} \mathrm{~T}_{r} I_{f} \mathrm{~T}_{r}}} \\
\triangle \log _{10} I_{f \mathrm{~T}}=\frac{1}{2.3 \sqrt{n_{f} m_{f \mathrm{~T}} I_{f} \mathrm{~T}}}
\end{gathered}
$$

where $n_{r}$ is the number of spectra the reference measurement was averaged over during acquisition (always 100), $n_{f}$ is the number of spectra that the full height measurements were averaged over during acquisition (given by Table 5.1) and $m_{f} \mathrm{~T}_{r}$ and $m_{f} \mathrm{~T}$ are the number of spectra that the absolute measurements at temperatures $\mathrm{T}_{r}$ and $\mathrm{T}$ were averaged over on exportation from the acquisition software to obtain the intensities $I_{f} \mathrm{~T}_{r}$ and $I_{f \mathrm{~T}}$ respectively. The value of $m_{f} \mathrm{~T}_{r}$ was always 100 and $m_{f} \mathrm{~T}$ was 18,16 and 15 for the ranges covering the 740,840 and $970 \mathrm{~nm}$ absorption bands respectively.

\section{A.2 Method II}

The expression for the absorption coefficient $\mu_{a \mathrm{~T}}$ calculated using Method II was given by Equation (5.22). Differentiating this expression with respect to each of the three variables gives:

$$
\begin{gathered}
\frac{\partial \mu_{a \mathrm{~T}}}{\partial \log _{10} I_{r}}=\frac{1}{h_{2 \mathrm{~T}_{S}}} \\
\frac{\partial \mu_{a \mathrm{~T}}}{\partial \log _{10} I_{f} \mathrm{~T}}=-\frac{1}{h_{2 \mathrm{~T}_{S}}}
\end{gathered}
$$




$$
\frac{\partial \mu_{a \mathrm{~T}}}{\partial h_{2 \mathrm{~T}_{S}}}=\frac{\log _{10} I_{f \mathrm{~T}}-\log _{10} I_{r}}{h_{2 \mathrm{~T}_{S}}^{2}}
$$

The error (squared) in the absorption coefficient is then given by

$$
\begin{gathered}
\left(\Delta \mu_{a \mathrm{~T}}\right)^{2}=\left(\frac{\partial \mu_{a \mathrm{~T}}}{\partial \log _{10} I_{r}}\right)^{2}\left(\Delta \log _{10} I_{r}\right)^{2}+\left(\frac{\partial \mu_{a \mathrm{~T}}}{\partial \log _{10} I_{f \mathrm{~T}}}\right)^{2}\left(\Delta \log _{10} I_{f \mathrm{~T}}\right)^{2} \\
+\left(\frac{\partial \mu_{a \mathrm{~T}}}{\partial h_{2 \mathrm{~T}}}\right)^{2}\left(\Delta h_{2 \mathrm{~T}_{S}}\right)^{2}
\end{gathered}
$$

where as before $\Delta \log _{10} I_{r}, \Delta \log _{10} I_{f} \mathrm{~T}$ and $\Delta h_{2} \mathrm{~T}_{S}$ are given by Equations (A.18), (A.20) and (A.11), and in this case $n_{r}$ was again 100, $n_{f}$ is given in Table 5.2 and $m_{f} \mathrm{~T}$ was 20 and 18 for the ranges $600-925 \mathrm{~nm}$ and $900-1050 \mathrm{~nm}$ respectively. 


\section{Appendix B}

\section{Eigenvector Proof for PCA}

As discussed in Section 5.3.5.1, in a principal component analysis (PCA) the data set $\mathbf{A}$ is decomposed into a series of $\mathrm{h}$ scores and loadings

$$
A=s_{1} \cdot l_{1}+s_{2} \cdot l_{2}+\ldots+s_{h} \cdot l_{h}+E_{A}
$$

This appendix will demonstrate that if one imposes the condition that the scores $\mathbf{s}_{i}$ are orthogonal to one another and likewise for the loadings $\mathbf{l}_{i}$, then the scores are the eigenvectors of $\left(\mathbf{A} \cdot \mathbf{A}^{\prime}\right)$ and the loadings are the eigenvectors of $\left(\mathbf{A}^{\prime} \cdot \mathbf{A}\right)$.

As follows from Equation (B.1), for the inclusion of only one PC the first score vector is given by

$$
\mathrm{s}_{1}=\mathbf{A} \cdot \mathbf{l}_{1}^{\prime} \cdot\left(\mathbf{l}_{1} \cdot \mathbf{l}_{1}^{\prime}\right)^{-1}
$$

and the loading vector is given by

$$
\mathbf{l}_{1}=\left(\mathbf{s}_{1}^{\prime} \cdot \mathbf{s}_{1}\right)^{-1} \cdot \mathbf{s}_{1}^{\prime} \cdot \mathbf{A}
$$

Let the scalars $\left(\mathrm{l}_{1} \cdot \mathrm{l}_{1}^{\prime}\right)$ and $\left(\mathrm{s}_{1}^{\prime} \cdot \mathrm{s}_{1}\right)$ equal $\mathrm{x}_{1}$ and $\mathrm{y}_{1}$ respectively. Equations (B.2) and (B.3) are now given by

$$
\begin{aligned}
& \mathbf{s}_{1} \cdot \mathbf{x}_{1}=\mathbf{A} \cdot \mathbf{l}_{1}^{\prime} \\
& \mathbf{l}_{1} \cdot \mathrm{y}_{1}=\mathbf{s}_{1}^{\prime} \cdot \mathbf{A}
\end{aligned}
$$

Upon substitution of Equation (B.5) into (B.4) one obtains

$$
\mathbf{s}_{1} \cdot \mathrm{x}_{1} \mathrm{y}_{1}=\left(\mathbf{A} \cdot \mathbf{A}^{\prime}\right) \cdot \mathbf{s}_{1}
$$


This corresponds to the classical eigenvector equation

$$
\mathbf{v} \cdot \lambda=\mathbf{M} \cdot \mathbf{v}
$$

in which the vector $\mathbf{v}$ is an eigenvector of the square matrix $\mathbf{M}$ with the eigenvalue $\lambda$. In the case of Equation (B.6) it is clear that the score vector $s_{1}$ is an eigenvector of the $(\mathrm{n} \times \mathrm{n})$ matrix $\left(\mathbf{A} \cdot \mathbf{A}^{\prime}\right)$ with an eigenvalue of $\mathrm{x}_{1} \mathrm{y}_{1}$. Similarly, substituting Equation (B.4) into (B.5) gives

$$
\mathbf{l}_{1} \cdot \mathrm{x}_{1} \mathrm{y}_{1}=\mathrm{l}_{1} \cdot\left(\mathbf{A}^{\prime} \cdot \mathbf{A}\right)
$$

i.e. the loading vector $l_{1}$ is an eigenvector of the $(m \times m)$ matrix $\left(\mathbf{A}^{\prime} \cdot \mathbf{A}\right)$.

Inclusion of a second PC will give the following equations for the second score and loading vectors

$$
\begin{aligned}
& \mathbf{s}_{2}=\left(A-\mathbf{s}_{1} \cdot \mathbf{l}_{1}\right) \cdot \mathbf{l}_{2}^{\prime} \cdot\left(\mathbf{l}_{2} \cdot \mathbf{l}_{2}^{\prime}\right)^{-1} \\
& \mathbf{l}_{2}=\left(\mathbf{s}_{2}^{\prime} \cdot \mathbf{s}_{2}\right)^{-1} \cdot \mathbf{s}_{2}^{\prime} \cdot\left(A-\mathbf{s}_{1} \cdot \mathbf{l}_{1}\right)
\end{aligned}
$$

Since $l_{2}$ is orthogonal to $l_{1}\left(\right.$ i.e. $l_{1} \cdot l_{2}^{\prime}=0$ ) and $s_{2}$ is orthogonal to $s_{1}\left(\right.$ i.e. $s_{1}^{\prime} \cdot s_{2}=0$ ), then Equations (B.9) and (B.10) become

$$
\begin{aligned}
& \mathbf{s}_{2}=\mathbf{A} \cdot \mathbf{l}_{2}^{\prime} \cdot\left(\mathbf{l}_{2} \cdot \mathbf{l}_{2}^{\prime}\right)^{-1} \\
& \mathbf{l}_{2}=\left(\mathbf{s}_{2}^{\prime} \cdot \mathbf{s}_{2}\right)^{-1} \cdot \mathbf{s}_{2}^{\prime} \cdot \mathbf{A}
\end{aligned}
$$

Equations (B.11) and (B.12) are identical to Equations (B.2) and (B.3), except that $\mathrm{s}_{2}$ and $l_{2}$ are substituted for $s_{1}$ and $l_{1}$. Following a similar argument to the case of only one PC given by Equations (B.5)-(B.8), it follows that $\mathbf{s}_{2}$ and $\mathbf{l}_{2}$ are also eigenvectors of $\left(\mathbf{A} \cdot \mathbf{A}^{\prime}\right)$ and $\left(\mathbf{A}^{\prime} \cdot \mathbf{A}\right)$ respectively, although with different eigenvalues. The same can be shown for the inclusion of up to $\mathrm{h}=\mathrm{m}$ PCs, the number of independent variables $\mathrm{m}$ being the maximum possible number of PCs. Thus the original statement, that imposing the condition of orthogonality on the scores and loadings determined by PCA will produce scores that are the eigenvectors of $\left(\mathbf{A} \cdot \mathbf{A}^{\prime}\right)$ and loadings that are the eigenvectors of $\left(\mathbf{A}^{\prime} \cdot \mathbf{A}\right)$, is proven. 


\section{Appendix C}

\section{Effect of Water Concentration on PCR Temperature Prediction}

In Section 5.3.5.1 the prediction of temperature from the spectral measurement of an 'unknown' sample using the PCR temperature calibration of the water absorption spectrum was described by Equations (5.45) and (5.46). As discussed in Section 5.3.5.2, however, the temperature prediction requires that the mean calibration spectrum (i.e. the mean spectrum of the calibration data set), is first subtracted from the 'unknown' spectrum at the concentration at which it should appear in the spectrum, i.e. at the water concentration of the unknown sample. Since the water concentration in the unknown sample is clearly unknown, it must be determined by including the mean calibration spectrum in the regression of the loading vectors to determine the scores. The effect of this on the subsequent temperature prediction is investigated in this appendix.

As follows from the discussion in Section 5.3.5.1, a mean-centered spectrum from the calibration data set, measured at any temperature, can be represented by its scores and loadings as

$$
\begin{aligned}
\mathbf{a}_{m c} & =\mathbf{a}-\overline{\mathbf{a}} \\
& =\mathbf{s} \cdot \mathbf{L}
\end{aligned}
$$

where $\mathbf{a}$ is the measured pure water spectrum, at a concentration of $100 \%$, and $\overline{\mathbf{a}}$ is the mean calibration spectrum (also at $100 \%$ ). In the prediction of temperature of a truly 'unknown' sample, the spectrum of the unknown sample is given by

$$
\mathbf{a}_{u}=c \cdot \mathbf{a}
$$


where $c$ is a fraction representing the concentration of the water in the sample relative to the pure water spectrum $\mathbf{a}$. The unknown spectrum $\mathbf{a}_{u}$ can be represented by the scores and loadings as follows

$$
\begin{aligned}
\mathbf{a}_{u} & =(\mathbf{s} \cdot \mathbf{L}+\overline{\mathbf{a}}) c \\
& =\mathbf{s}^{*} \cdot \mathbf{L}^{*}
\end{aligned}
$$

where $\mathbf{s}^{*}$ and $\mathbf{L}^{*}$ are the modified scores and loadings, the components of which are given by

$$
\begin{aligned}
\mathbf{s}^{*} & =\left[\mathrm{s}_{1} c \mathrm{~s}_{2} c \cdots \mathrm{s}_{h} c\right] \\
\mathbf{L}^{*} & =\left[\begin{array}{ll}
\mathbf{l}_{1} & \mathbf{l}_{2} \cdots \mathbf{l}_{h} \\
\mathbf{a}
\end{array}\right]
\end{aligned}
$$

i.e. the first $\mathrm{h}$ components of $\mathrm{s}^{*}$ are the original scores for the $\mathrm{h}$ PCs included in the analysis multiplied by the concentration $c$, and the final component is the value of $c$, and the first $h$ components of $\mathbf{L}^{*}$ are the original loading vectors for the $\mathrm{h}$ PCs (at $\mathrm{m}$ wavelengths) and the last component is the mean calibration spectrum $\overline{\mathbf{a}}$. Since the mean calibration spectrum is not orthogonal to the loading vectors, i.e. $\left(\mathbf{L}^{*} \cdot \mathbf{L}^{*^{\prime}}\right) \neq \mathbf{I}$, the modified scores must be determined in the least-squares sense by the full regression of $\mathbf{a}_{u}$ on to $\mathbf{L}^{*}$

$$
\hat{\mathbf{s}}^{*}=\mathbf{a}_{u} \cdot \mathbf{L}^{*^{\prime}} \cdot\left(\mathbf{L}^{*} \cdot \mathbf{L}^{*^{\prime}}\right)^{-1}
$$

The scores are then recovered by normalising the first $\mathrm{h}$ components of $\hat{\mathbf{s}}^{*}$ to the concentration $c$ given by the last component of $\hat{\mathbf{s}}^{*}$

$$
\hat{\mathbf{s}}=\frac{\hat{\mathbf{s}}^{*}(1,2, \cdots h)}{\hat{\mathbf{s}}^{*}(h+1)}
$$

Due to the non-orthogonality of the components in the modified loadings matrix $\mathbf{L}^{*^{\prime}}$, the least-squares regression in Equation (C.5) will not yield the exact values for either the concentration $c$ of the mean calibration spectrum or the product of $c$ and the original scores. Normalising the modified scores by the concentration $c$ as in Equation (C.6) will not, therefore, precisely recover the original scores, i.e. $\hat{\mathbf{s}} \neq \mathbf{s}$. This will translate into an error in the predicted temperature, relative to that determined with the correct scores, when the normalised scores are multiplied by the original calibration vector $\hat{\mathbf{v}}$ as in Equation (5.46). 


\begin{tabular}{llll}
\hline & $\mathrm{PCs}$ & $\mathrm{SEC}\left({ }^{\circ} \mathrm{C}\right)$ & $r_{c}^{2}$ \\
\hline Original & 1 & 0.1360 & 0.9988 \\
calibration & 2 & 0.1053 & 0.9993 \\
& 3 & 0.1008 & 0.9993 \\
& 4 & 0.1003 & 0.9994 \\
\hline Concentration & 1 & 0.2595 & 0.9957 \\
corrected & 2 & 0.1066 & 0.9993 \\
& 3 & 0.1076 & 0.9993 \\
& 4 & 0.1025 & 0.9993 \\
\hline
\end{tabular}

Table C.1: Statistical parameters for the PCR calibration of the temperature-matched ranges of the water absorption spectrum measured by Method I.

The temperature was predicted from the normalised scores, determined in the manner described above, using the water absorption spectra measured by Method I (see Section 5.1.2.1) in the range $650-805 \mathrm{~nm}$ at various different 'water concentrations', simulated by multiplying the data set by the required concentration, but fitting the mean calibration spectrum at a concentration of $100 \%$. The standard errors on the estimated temperature, given in Table C.1, did not vary with the assumed water concentration. For comparison, the standard errors determined in the original calibration are also included. In general, the errors in the temperature prediction are greater when correcting for the concentration by fitting the mean calibration spectrum, although the relative differences between them decrease as the number of PCs included increases. 


\section{Appendix D}

\section{Orthogonality Proof for PLSR}

As described in Section 5.3.6.1 the orthogonalised PLSR algorithm involves the additional step of defining a loading vector as well as a weight vector. This, in conjunction with the iterative step of using the residuals from the $i-1$ th factor to calculate the parameters for the $i$ th factor, ensure that the scores are orthogonal to one another, as will be demonstrated here.

The first score vector was defined as $\mathbf{s}_{1}=\mathbf{A} \cdot \mathbf{w}_{1}^{\prime}$. From the definition of the residual matrix given in Equation (5.51), the second score vector for the 'orthogonalised' method is given by

$$
\begin{aligned}
\mathbf{s}_{2} & =\mathbf{A}_{1} \cdot \mathbf{w}_{2}^{\prime} \\
& =\left(\mathbf{A}-\mathbf{s}_{1} \cdot \mathbf{l}_{1}\right) \cdot \mathbf{w}_{2}^{\prime}
\end{aligned}
$$

If the score vectors $\mathbf{s}_{1}$ and $\mathbf{s}_{2}$ are orthogonal then $\mathbf{s}_{1}^{\prime} \cdot \mathbf{s}_{2}=0$. Multiplying Equation (D.1) on the left by $s_{1}^{\prime}$ gives

$$
\mathbf{s}_{1}^{\prime} \cdot \mathbf{s}_{2}=\mathbf{s}_{1}^{\prime} \cdot\left(\mathbf{A}-\mathbf{s}_{1} \cdot \mathbf{l}_{1}\right) \cdot \mathbf{w}_{2}^{\prime}
$$

Clearly, if the score vectors are orthogonal then the left hand side of Equation (D.2) will be zero, which means that the following must be true

$$
\mathbf{s}_{1}^{\prime} \cdot \mathbf{A} \cdot \mathbf{w}_{2}^{\prime} \equiv \mathbf{s}_{1}^{\prime} \cdot \mathbf{s}_{1} \cdot \mathbf{l}_{1} \cdot \mathbf{w}_{2}^{\prime}
$$

On substituting $\mathbf{l}_{1}$, given by $\mathbf{l}_{1}=\left(\mathbf{s}_{1}^{\prime} \cdot \mathbf{s}_{1}\right)^{-1} \cdot \mathbf{s}_{1}^{\prime} \cdot \mathbf{A}$, into Equation (D.3) above, the right hand side becomes

$$
\left(\mathbf{s}_{1}^{\prime} \cdot \mathbf{s}_{1}\right) \cdot\left(\mathbf{s}_{1}^{\prime} \cdot \mathbf{s}_{1}\right)^{-1} \cdot \mathbf{s}_{1}^{\prime} \cdot \mathbf{A} \cdot \mathbf{w}_{2}^{\prime}
$$

The first two terms in parentheses cancel one another out, leaving $\mathbf{s}_{1}^{\prime} \cdot \mathbf{A} \cdot \mathbf{w}_{2}^{\prime}$, which is 
identical to the left hand side of (D.3). Hence the first two score vectors are orthogonal, as can be shown by a similar argument for all subsequent scores. Using this method the weight vectors are also orthogonal, but, unlike PCR, the loading vectors are not. 


\section{References}

Agah, R, Gandjbakhche, A H, Motamedi, M, Nossal, R, and Bonner, R F. 1996. Dynamics of temperature dependent optical properties of tissue: dependence on thermally induced alteration. IEEE Trans Biomed Eng, 43(8), 839-846.

Alberts, B, Bray, D, Lewis, J, Raff, M, Roberts, K, and Watson, J D. 1983. Molecular biology of the cell. New York: Garland Publishing. chapter 7, p322.

Arnfield, M R, Mathew, R P, Tulip, J, and McPhee, M S. 1992. Analysis of tissue optical coefficients using an approximate equation valid for comparable absorption and scattering. Phys Med Biol, 37(6), 1219-1230.

Arridge, S R, Cope, M, and Delpy, D T. 1992. The theoretical basis for the determination of optical pathlengths in tissue: temporal and frequency analysis. Phys Med Biol, 37(7), 1531-1560.

Azzopardi, D, Wyatt, J S, Cady, E B, Delpy, D T, Baudin, J, Stewart, A L, Hope, P L, Hamilton, P A, and Reynolds, E O. 1989. Prognosis of newborn infants with hypoxicischemic brain injury assessed by phosphorus magnetic resonance spectroscopy. $P e$ diatr Res, 25(5), 445-451.

Baena, R C, Busto, R, Dietrich, W D, Globus, M Y, and Ginsberg, M D. 1997. Hyperthermia delayed by 24 hours aggravates neuronal damage in rat hippocampus following global ischemia. Neurology, 48(3), 768-773.

Bayly, J G, Kartha, V B, and Stevens, W H. 1963. The absorption spectra of liquid phase $\mathrm{H}_{2} \mathrm{O}, \mathrm{HDO}$ and $\mathrm{D}_{2} \mathrm{O}$ from $0.7 \mu \mathrm{m}$ to $10 \mu \mathrm{m}$. Infrared Physics, 3, 211-223.

Beauvoit, B, and Chance, B. 1998. Time-resolved spectroscopy of mitochondria, cells and tissues under normal and pathological conditions. Mol Cell Biochem, 184, 445-455.

Beauvoit, B, Evans, S M, Jenkins, T W, Miller, E E, and Chance, B. 1995. Correlation 
between the light scattering and the mitochondrial content of normal tissues and transplantable rodent tumors. Anal Biochem, 226, 167-174.

Beek, J F, Blokland, P, Posthumus, P, Aalders, M, and Pickering, J W. 1997. In vitro double-integrating sphere optical properties of tissues between 630 and $1064 \mathrm{~nm}$. Phys Med Biol, 42, 2255-2261.

Beer, A. 1852. Bestimmung der Absorption des rothen Lichts in farbigen Flüssigketiten. Ann Physik Chem, 86(5), 78-88.

Benedict, W S, Gailar, N, and Plyler, E K. 1956. Rotation-vibration spectra of deuterated water vapour. J Chem Phys, 24(6), 1139-1165.

Bertsch, F, Mattner, J, Stehling, M K, Müller-Lisse, U, Peller, M, Loeffler, R, Weber, J, Meßmer, K, Wilmanns, W, Issels, R, and M, Reiser. 1998. Non-invasive temperature mapping using MRI: comparison of two methods based on chemical shift and $\mathrm{T}_{1^{-}}$ relaxation. Mag Res Imag, 16(4), 393-404.

Beuthan, J, Minet, O, Helfman, J, Herrig, M, and Müller, G. 1996. The spatial variation of the refractive index in biological cells. Phys Med Biol, 41, 369-382.

Bevilacqua, F, Marquet, P, Depeursinge, C, and de Haller, E B. 1995. Determination of reduced scattering and absorption coefficients by a single charge-coupled-device array measurement, part II: measurement on biological tissues. Opt Eng, 34(7), 2064-2069.

Bigelow, W G, Lindsay, W K, and Greenwood, W F. 1950. Hypothermia: Its possible role in cardiac surgery. Ann Surg, 132, 849-866.

Binzoni, T, Hiltbrand, E, Kayser, B, Ferretti, G, and Terrier, F. 1995. Human intramuscular temperature and heat flow transients at rest. J Appl Physiol, 79(5), 1736-1743.

Binzoni, T, Springett, R, Dalton, J C, and Delpy, D T. 1999. A new combined deepbody-temperature/NIRS probe for non-invasive metabolic measurements on human skeletal muscle. Adv Exp Med Biol, 471, 623-629.

Bohren, C F, and Huffman, D R. 1983. Absorption and scattering of light by small particles. First edn. New York: John Wiley and Sons.

Bolin, F P, Preuss, L E, Taylor, R C, and Ference, R J. 1989. Refractive index of some mammalian tissues using a fiber optic cladding method. Appl Opt, 28(12), 2297-2303. 
Bolte, R G, Black, P G, Bowers, R S, Thorne, J K, and Corneli, H M. 1988. The use of extracorporeal rewarming in a child submerged for 66 minutes. JAMA, 260(3), 377-379.

Bonner, O D, Arisman, R K, and Jumper, C F. 1977. The effect of organic solutes on water structure. $Z$ phys Chemie, Leipzig, 1, 49-58.

Bouguer, P. 1729. Essai d'optique sur la gradation de la lumière. Section II. De la transparence et de l'opacité. II. De la proportion selon laquelle la lumière diminué en traversant les milieux.

Buijs, K, and Choppin, G R. 1963. Near-infrared studies of the structure of water. I. Pure water. J Chem Phys, 39(8), 2035-2041.

Buijs, K, and Choppin, G R. 1964. Assignment of the near-infrared bands of water and ionic solutions. J Chem Phys, 40, 3120.

Buontempo, U, Careri, G, and Fasella, P. 1972. Hydration water of globular proteins: The infrared band near $3300 \mathrm{~cm}^{-1}$. Biopolymers, 11, 519-521.

Cady, E B, D'Souza, P C, Penrice, J, and Lorek, A. 1995. The estimation of local brain temperature by in vivo ${ }^{1} \mathrm{H}$ magnetic resonance spectroscopy. Mag Res Med, 33, 862867.

Campistol, J, Poo, P, Fernandez Alvarez, E, and Carratala, F. 1999. Parasagittal cerebral injury: magnetic resonance findings. $J$ Child Neurol, 14(10), 683-685.

Carter, D L, MacFall, J R, Clegg, S T, Wan, X, Prescott, D M, Charles, H C, and Samulski, T V. 1998. Magnetic resonance thermometry during hyperthermia for human highgrade sarcoma. Int J Radiat Oncol Biol Phys, 40(4), 815-822.

Case, K M, and Zweifel, P F. 1967. Linear transport theory. Reading (Mass): AddisonWesle.

Chadwick, R S, and Chang, I D. 1973. A laser study of the motion of particles suspended in a slow viscous shear flow. $J$ Colloid Interface Sci, 42, 516-534.

Chance, B, Maris, M, Sorge, J, and Zhang, M Z. 1990. A phase modulation system for dual wavelength difference spectroscopy of hemoglobin deoxygenation in tissues. Proc SPIE, 1204, 481-491. 
Chance, B, Liu, H, Kitai, T, and Zhang, Y. 1995. Effects of solutes on optical properties of biological materials: models, cells and tissues. Anal Biochem, 227, 351-362.

Cheever, E A, and Foster, K R. 1992. Microwave radiometry in living tissue: what does it measure? IEEE Trans Biomed Eng, 39(6), 563-568.

Cheong, W, Prahl, S A, and Welch, A J. 1990. A review of the optical properties of biological tissues. IEEE J Quant Elec, 26(12), 2166-2185.

Childs, C, Harrison, R, and Hodkinson, C. 1999. Tympanic membrane temperature as a measure of core temperature. Arch Dis Child, 80, 262-266.

Chong, C S, and Colbow, K. 1976. Light scattering and turbidity measurements on lipid vesicles. Biochim Biophys Acta, 436, 260-282.

Colbourne, F, and Corbett, D. 1994. Delayed and prolonged post-ischemic hypothermia is neuroprotective in the gerbil. Brain Res, 654(2), 265-272.

Colbourne, F, and Corbett, D. 1995. Delayed postischemic hypothermia: a six month survival study using behavioural and histological assessments of neuroprotection. $J$ Neurosci, 15(11), 7520-7560.

Colbourne, F, Sutherland, G, and Corbett, D. 1997. Postischemic hypothermia. A critical appraisal with implications for clinical treatment. Mol Neurobiol, 14(3), 171-201.

Contini, D, Martelli, F, and Zaccanti, G. 1997. Photon migration through a turbid slab described by a model based on diffusion approximation. I. Theory. Appl Opt, 36(19), $4587-4599$.

Conway, J M, Norris, K H, and Bodwell, C E. 1984. A new approach for the estimation of body composition: infrared interactance. Am J Clin Nutr, 40(6), 1123-1130.

Cooper, C E, Elwell, C E, Meek, J H, Matcher, S J, Wyatt, J S, Cope, M, and Delpy, D T. 1996. The non-invasive measurement of absolute cerebral deoxyhaemoglobin concentration and mean optical path length in the neonatal brain by second derivative NIRS. Ped Res, 39(1), 32-38.

Cooper, C E, Cope, M, Springett, R, Amess, P N, Penrice, J, Tyszczuk, L, Punwani, S, Ordidge, R, Wyatt, J S, and Delpy, D T. 1999. Use of mitochondrial inhibitors to demonstrate that cytochrome oxidase near-infrared spectroscopy can measure mitochondrial dysfunction noninvasively in the brain. J Cereb Blood Flow Metab, 19(1), 27-38. 
Cope, M. 1991. The development of a near infrared spectroscopy system and its application for non invasive monitoring of cerebral blood and tissue oxygenation in the newborn infant. $\mathrm{PhD}$, University College London, London.

Cordone, L, Cupane, A, Leone, M, and Vitrano, E. 1986. Optical absorption spectra of deoxy- and oxyhaemoglobin in the temperature range 300-20 K. Relation with protein dynamics. Biophys Chem, 24, 259-275.

Craig, J V, Lancaster, G A, Williamson, P R, and Smyth, R L. 2000. Temperature measured at the axilla compared with rectum in children and young people: a systematic review. $B M J, \mathbf{3 0}, 1174-1178$.

Cubeddu, R, Pifferi, A, Taroni, P, Torricelli, A, and Valentini, G. 1999. Compact tissue oximeter based on dual-wavelength multichannel time-resolved reflectance. Appl Opt, 38(16), 3670-3680.

de Jode, M L. 1999. Monte Carlo simulations of the use of isotropic light dosimetry probes to monitor energy fluence in biological tissues. Phys Med Biol, 44, 3027-3037.

de Poorter, J, de Wagter, C, de Deene, Y, Thomson, C, Stahlberg, F, and Achten, E. 1995. Non-invasive MRI thermometry with the proton resonance frequency (prf) method: in vivo results in human muscle. Mag Res $M e d, \mathbf{3 3}, 74-81$.

Del Bianco, A, Benes, R, Trinkel, M, Reininger, F, and M, Leitner. 2000. Temperature influence on the 1.2 $\mu \mathrm{m}$ band of water. Near infrared spectroscopy: Proc 9th Int Conference.

Delannoy, J, Chen, C N, Turner, R, Levin, R L, and Le Bihan, D. 1991. Noninvasive temperature imaging using diffusion MRI. Mag Res Imag, 19, 333-339.

Delpy, D T, Cope, M, van der Zee, P, and Arridge, S. 1988. Estimation of optical pathlength through tissue from direct time of flight measurement. Phys Med Biol, 248, $41-46$.

Dickinson, D F, and Sambrooks, J E. 1979. Intellectual performance in children after circulatory arrest with profound hypothermia in infancy. Arch Dis Child, 54(1), 1-6.

Dollberg, S, Xi, Y, and Donnelly, M M. 1993. A noninvasive transcutaneous alternative to rectal thermometry for continuous measurement of core temperature in the piglet. Ped Res, 34(4), 512-517. 
Doornbos, R M, Lang, R, Aalders, M C, Cross, F W, and J, Sterenborg H. 1999. The determination of in vivo human tissue optical properties and absolute chromophore concentrations using spatially resolved steady-state diffuse reflectance spectroscopy. Phys Med Biol, 44, 967-981.

Dubois, L, Pribetich, J, Favre, J J, Chivé, M, and Moschetto, Y. 1993. Non-invasive microwave multifrequency radiometry used in microwave hyperthermia for bidimensional reconstruction of temperature patterns. Int $J$ Hyperthermia, 9(3), 415-431.

Duncan, A, Whitlock, T, Cope, M, and Delpy, D T. 1993. A multiwavelength, wideband, intensity-modulated spectrometer for near-IR spectroscopy and imaging. Proc SPIE, 1888, 248-257.

Eason, G, Veitch, R, Nisbet, R, and Turnbull, R. 1978. The theory of back-scattering of light in blood. $J$ Phys $D, 11,1463-1479$.

Edrich, J, Jobe, W E, Cacak, R K, Hendee, W R, Smyth, C J, Gautherie, M, Gros, C, Zimmer, R, Robert, J, Thouvenot, P, Escanye, J M, and Itty, C. 1980. Imaging thermograms at centimeter and millimeter wavelengths. Ann NY Acad Sci, 335, 456-474.

Edwards, A D, and Azzopardi, D. 1998. Hypothermic neural rescue treatment: from laboratory to cotside? Arch Dis Child Fetal Neonatal Ed, 78, F88-F91.

Edwards, A D, and Mehmet, H. 1996. Apoptosis in perinatal hypoxic-ischaemic cerebral damage. Neuropathol Appl Neurobiol, 22(6), 494-498.

Edwards, A D, Wyatt, J S, and Thoresen, M. 1998. Treatment of hypoxic-ischaemic brain damage by moderate hypothermia. Arch Dis Child Fetal Neonatal Ed, 78, F85-F88.

Eisenberg, D, and Kauzmann, W. 1969. Structure and properties of water. Oxford: Oxford University Press.

Essenpreis, M. 1992. Thermally induced changes in optical properties of biological tissues. $\mathrm{PhD}$, University College London, London.

Essenpreis, M, Elwell, C E, Cope, M, van der Zee, P, Arridge, S R, and Delpy, D T. 1993. Spectral dependence of temporal point spread functions in human tissues. Appl Opt, 32(4), 418-425. 
Falk, M, and Ford, T A. 1966. Infrared spectrum and structure of liquid water. Can J Chem, 44, 1699-1707.

Fantini, S, Hueber, D, Franceschini, M A, Gratton, E, Rosenfeld, W, Stubblefield, P G, Maulik, D, and Stankovic, M R. 1999. Non-invasive optical monitoring of the newborn piglet brain using continuous-wave and frequency-domain spectroscopy. Phys Med Biol, 44, 1543-1563.

Farahani, K, Saxton, R E, Yoon, H C, De Salles, A A, Black, K L, and Lufkin, R B. 1999. MRI of thermally denatured blood: methemoglobin formation and relaxation effects. Mag Res Imag, 17(10), 1489-1494.

Farrell, T J, and Patterson, M S. 1992. A diffusion theory model of spatially resolved, steady-state diffuse reflectance for the noninvasive determination of tissue optical properties in vivo. Med Phys, 19(4), 879-888.

Fearn, T. 1999. A look at some standard pre-treatments for spectra. NIR News, 10, 10-11.

Fellman, V, and Raivio, K O. 1997. Reperfusion injury as the mechanism of brain damage after perinatal asphyxia. Ped Res, 41(5), 599-606.

Ferrara-Love, R. 1991. A comparison of tympanic and pulmonary artery measures of core temperatures. J Post Anesth Nurs, 6(3), 161-164.

Ferretti, G, Binzoni, T, Hulo, N, Kayser, B, Thomet, J, and Cerretelli, P. 1995. Kinetics of oxygen consumption during maximal exercise at different muscle temperatures. Resp Physiol, 102, 261-268.

Firbank, M, Hiraoka, M, Essenpreis, M, and Delpy, D T. 1993. Measurement of the optical properties of the skull in the wavelength range 650-950 nm. Phys Med Biol, 38, 503-510.

Firbank, M, Arridge, S R, Schweiger, M, and Delpy, D T. 1996. An investigation of light transport through scattering bodies with non-scattering regions. Phys Med Biol, 41, 767-783.

Flock, S T, Wilson, B C, and Patterson, M S. 1987. Total attenuation coefficients and scattering phase functions of tissues and phantom materials at $633 \mathrm{~nm}$. Med Phys, 14(5), 835-841. 
Flock, S T, Patterson, M S, Wilson, B C, and Wyman, D R. 1989. Monte Carlo modeling of light propagation in highly scattering tissues-I: Model predictions and comparison with diffusion theory. IEEE Trans Biomed Eng, 36(12), 1162-1168.

Foster, K R, and Cheever, E A. 1992. Microwave radiometry in biomedicine: a reappraisal. Bioelectromagnetics, 13, 567-579.

Fox, R H, and Solman, A J. 1971. A new technique for monitoring the deep body temperature in man from the intact skin surface. $J$ Physiol, 212, 8P-10P.

Franceschini, M A, Fantini, S, Paunescu, L A, Maier, J S, and Gratton, E. 1998. Influence of a superficial layer in the quantitative spectroscopic study of strongly scattering media. Appl Opt, 37(31), 7447-7458.

Frenzel, T, Roth, K, Koßler, S, Radüchel, B, Bauer, H, Platzek, J, and Weinmann, H. 1996. Noninvasive temperature measurement in vivo using a temperature-sensitive lanthanide complex and ${ }^{1} \mathrm{H}$ magnetic resonance spectroscopy. Mag Res Med, 35, 364-369.

Fulbrook, P. 1997. Core body temperature measurement: a comparison of axilla, tympanic membrane and pulmonary artery blood temperature. Intensive Crit Care Nurs, 13(5), $266-72$.

Gans, R. 1925. Strahlungsdiagramme ultramikroskopischer Teilchen. Ann Physik, 76, $29-38$.

Geladi, P, and Kowalski, B R. 1986. Partial least-squares regression: A tutorial. Anal Chim Acta, 185, 1-17.

Gill, P E, Murray, W, and Wright, M H. 1981. Practical optimization. London, UK: Academic Press.

Ginsberg, M D, Sternau, L L, Globus, M Y, Dietrich, W D, and Busto, R. 1992. Therapeutic modulation of brain temperature: relevance to ischemic brain injury. Cerebrovasc Brain Metab Rev, 4(3), 189-225.

Gniadecka, M, Nielsen, O F, Christensen, D H, and Wulf, H C. 1998. Structure of water, proteins and lipids in intact human skin, hair and nail. $J$ Invest Dermatol, 110, 393-398. 
Godik, E E, and Gulyaev, Y V. 1991. Functional imaging of the human body. IEEE Eng Med Biol, 10(4), 21-29.

Greeley, W J, Ungerleider, R M, Smith, L R, and Reves, J G. 1989. The effects of deep hypothermic cardiopulmonary bypass and total circulatory arrest on cerebral blood flow in infants and children. $J$ Thorac Cardiovasc Surg, 97(5), 737-745.

Gunn, A J, and Gunn, T R. 1996. Effect of radiant heat on head temperature gradient in term infants. Arch Dis Child, 74, F200-F203.

Gunn, A J, Gluckman, P D, and Gunn, T R. 1998. Selective head cooling in newborn infants after perinatal asphyxia: a safety study. Pediatrics, 102(4 Pt 1), 885-892.

Gustov, A V, Troitskii, V S, Gorbachev, V P, Arzhanov, N I, and Tseitlina, V N. 1985. Investigation of craniocerebral temperature by decimeter radiothermometry. Fiziologiya Cheloveka, 11(1), 151-154.

Haaland, D M, and Thomas, E V. 1988. Partial least-squares methods for spectral analyses. 1. Relation to other quantitative calibration methods and the extraction of qualitative information. Anal Chem, 60, 1193-1202.

Haaland, D M, Easterling, R G, and Vopicka, D A. 1985. Multivariate least-squares methods applied to the quantitative spectral analysis of multicomponent samples. Applied Spectroscopy, 39(1), 73-84.

Haaland, K, Steen, P A, and Thoreson, M. 1996. Cerebral, tympanic and colonic thermometry in the piglet. Reprod Fertil Dev, 8(1), 125-128.

Haddock, B J, Merrow, D L, and Swanson, M S. 1996. The falling grace of axillary temperatures. Pediatr Nurs, 22(2), 121-125.

Hale, G M, and Querry, M R. 1973. Optical constants of water in the 200-nm to $200-\mu \mathrm{m}$ wavelength region. Appl Opt, 12(3), 555-563.

Hand, J W, Van Leeuwen, G M, Mizushina, S, Van de Kamer, J B, Maruyama, K, Sugiura, T, Azzopardi, D V, and Edwards, A D. 2001. Monitoring of deep brain temperature in infants using multi-frequency microwave radiometry and thermal modelling. Phys Med Biol, 46, 1885-1903.

Harioka, T, Matsukawa, T, Ozaki, M, Nomura, K, Sone, T, Kakuyama, M, and Toda, H. 2000. "Deep-forehead" temperature correlates well with blood temperature. Can J Anaesth, 47(10), 98-983. 
Helland, I S. 1988. On the structure of partial least squares regression. Commun Statist - Simula Computa, 17(2), 581-607.

Hielscher, A H, Liu, H, Chance, B, Tittel, F K, and Jacques, S L. 1996. Time-resolved photon emission from layered turbid media. Appl Opt, 35(4), 719-728.

Hillman, E M, Hebden, J C, Schweiger, M, Dehghani, H, Schmidt, F E, Delpy, D T, and Arridge, S R. 2001. Time resolved optical tomography of the human forearm. Phys Med Biol, 46, 1117-1130.

Hindman, J C. 1966. Proton resonance shift of water in the gas and liquid states. $J$ Chem Phys, 44(12), 4582-4592.

Hiraoka, M, Firbank, M, Essenpreis, M, M, Cope, Arridge, S R, van der Zee, P, and Delpy, D T. 1993. A Monte Carlo investigation of optical pathlength in inhomogeneous tissue and its application to near-infrared spectroscopy. Phys Med Biol, 38, 1859-1876.

Hirschfield, T. 1985. Salinity determination using NIRA. Appl Spec, 39(4), 740-741.

Hollis, V S, Binzoni, T, and Delpy, D T. 2001. Non-invasive monitoring of brain tissue temperature by near-infrared spectroscopy. SPIE Proc, 4250, 470-481.

Hornig, D F. 1964. On the spectrum and stucture of water and ionic solutions. J Chem Phys, 40, 3119-3120.

Hueber, D M, Franceschini, M A, Ma, H Y, Zhang, Q, Ballesteros, J R, Fantini, S, Wallace, D, Ntziachristos, V, and Chance, B. 2001. Non-invasive and quantitative near-infrared haemoglobin spectrometry in the piglet brain during hypoxic stress, using a frequency-domain multidistance instrument. Phys Med Biol, 46, 41-62.

Huh, P W, Belayev, L, Zhao, W, Koch, S, Busto, R, and Ginsberg, M D. 2000. Comparative neuroprotective efficacy of prolonged moderate intraischemic and postischemic hypothermia in focal cerebral ischemia. $J$ Neurosurg, 92(1), 91-99.

Hull, E L, Nichols, M G, and Foster, T H. 1998. Quantitative broadband near-infrared spectroscopy of tissue-simulating phantoms containing erythrocytes. Phys Med Biol, 43, 3381-3404.

Ishimaru, A. 1978. Wave propagation and scattering in random media. New York: Academic Press. 
Jaywant, S, Wilson, B, Patterson, M, Lilge, L, Flotte, T, Woolsey, J, and McCulloch, C. 1993 . Temperature dependent changes in the optical absorption and scattering spectra of tissues: correlation with ultrastructure. Proc SPIE, 1882, 218-229.

Jendrasiak, G L. 1996. The hydration of phospholipids and its biological significance. Nutr Biochem, 7, 599-609.

Jenkins, F A, and White, H E. 1981. Fundamentals of optics. Fourth edn. New York: McGraw-Hill.

Jöbsis, F F. 1977. Noninvasive, infrared monitoring of cerebral and myocardial oxygen sufficiency and circulatory parameters. Science, 198, 1264-1267.

Karmakar, A K, and Joarder, R N. 1994. A simple cluster model for the description of liquid water: temperature effects. Chem Phys, 183, 11-17.

Kelly, J J, Kelly, K A, and Barlow, C H. 1995. Tissue temperature by near-infrared spectroscopy. SPIE Proc: Optical tomography, photon migration and spectroscopy of tissue and model media: Theory, human studies and instrumentation.

Kienle, A, and Patterson, M S. 1997. Improved solutions of the steady-state and timeresolved diffusion equations for reflectance from a semi-infinite turbid medium. $J$ Opt Soc Am A, 14(1), 246-254.

Kienle, A, Lilge, L, Patterson, M, Raimund, H, Steiner, R, and Wilson, B. 1996. Spatially resolved absolute diffuse reflectance measurements for noninvasive determination of the optical scattering and absorption coefficients of biological tissue. Appl Opt, 35(13), 2304-2314.

Kohl, M, Watson, R, and Cope, M. 1997. Optical properties of highly scattering media determined from changes in attenuation, phase and modulation depth. Appl Opt, 36(1), 105-114.

Kohl, M, Nolte, C, Heekeren, H, Horst, Susanne, Scholz, U, Obrig, H, and Villringer, A. 1998. Determination of the wavelength dependence of the differential pathlength factor from near-infrared pulse signals. Phys Med Biol, 43, 1771-1782.

Kurth, C D, Liu, H, Thayer, W S, and Chance, B. 1995. A dynamic phantom brain model for near-infrared spectroscopy. Phys Med Biol, 40, 2079-2092. 
Lambert, J H. 1760. Photometria, sive de mensura et gradibus luminis, colorum et umbrae. Augsburg.

Langford, V S, McKinley, A J, and Quickenden, T I. 2001. Temperature dependence of the visible-near-infrared absorption spectrum of liquid water. J Phys Chem A, 105(39), 8916-8921.

Lanham, D M, Walker, B, Klocke, E, and Jennings, M. 1999. Accuracy of tympanic temperature readings in children under 6 years of age. Pediatr Nurs, 25(1), 39-42.

Laufer, J, Simpson, R, Kohl, M, Essenpreis, M, and Cope, M. 1998. Effect of temperature on the optical properties of ex vivo human dermis and subdermis. Phys Med Biol, 43, 2479-2489.

Leroy, Y, Bocquet, B, and Mamouni, A. 1998. Non-invasive microwave radiometry thermometry. Physiol Meas, 19, 127-148.

Libnau, F O, Kvalheim, O M, Christy, A A, and Toft, J. 1994. Spectra of water in the near and mid-infrared region. Vib Spec, 7, 243-254.

Lin, J, and Brown, C W. 1993. Near-IR fibre-optic temperature sensor. Appl Spec, 47(1), $62-68$.

Liu, H, Boas, D A, Zhang, Y, Yodh, A G, and Chance, B. 1995. Determination of optical properties and blood oxygenation in tissue using continuous NIR light. Phys Med Biol, 40, 1983-1993.

Lorek, A, Takei, Y, Cady, E B, Wyatt, J S, Penrice, J, Edwards, A D, Peebles, D, Wylezinska, M, Owen-Reece, H, and Kirkbride, V. 1994. Delayed ("secondary") cerebral energy failure after acute hypoxia-ischemia in the newborn piglet: continuous 48-hour studies by phosphorus magnetic resonance spectroscopy. Pediatr Res, 36(6), 699-706.

Lovell, A T, Hebden, J C, Goldstone, J C, and Cope, M. 1999. Determination of the transport scattering coefficient of red blood cells. SPIE Proc: Optical Tomography and Spectroscopy of Tissue III, 3597, 175-182.

Maass-Moreno, R, Damianou, C A, and Sanghvi, N T. 1996. Noninvasive temperature estimation in tissue via ultrasound echo-shifts. Part II. In vitro study. J Acoust Soc $A m, 100(4$ Pt 1), 2522-2530. 
Mantsch, H H, Madec, C, Lewis, R N, and McElhaney, R N. 1985. Thermotropic phase behavior of model membranes composed of phosphatidylcholines containing iso-branched fatty acids. 2. Infrared and 31P NMR spectroscopic studies. Biochem, 24(10), 2440-2446.

Mantsch, H H, Madec, C, Lewis, R N, and McElhaney, R N. 1987. Thermotropic phase behaviour of model membranes composed of phosphatidylcholines containing $d l$-methyl anteisobranched fatty acids. 2. An infrared spectroscopic study. Biochem, 26(13), 4045-4049.

Marchesini, R, Clemente, C, Pignoli, E, and Brambilla, M. 1992. Optical properties of in vitro epidermis and their possible relationship with optical properties of in vivo skin. $J$ Photochem Photobiol B, 16(2), 127-140.

Marcus, Y. 1995. Water binding by organic molecules. Cell Biochem Func, 13, 157-163.

Mariak, Z, Lewko, J, Luczaj, J, Polocki, B, and D, White M. 1994. The relationship between directly measured human cerebral and tympanic temperatures during changes in brain temperatures. Eur J Appl Physiol Occup Physiol, 69(6), 545-549.

Marieb, E N. 1995. Human Anatomy and Physiology. Third edn. Redwood City, California: Benjamin/Cummings.

Marquez, G, Wang, L V, Lin, S, Schwartz, J A, and Thomsen, S L. 1998. Anisotropy in the absorption and scattering spectra of chicken breast tissue. Appl Opt, 37(4), 798-804.

Martens, H. 2000. Personal communication. Norwegian University of Science and Technology (NTNU), Trondheim, Norway.

Martens, H, and Næs, T. 1991. Multivariate Calibration. Chichester: John Wiley and Sons.

Martin, K A, and Curtis, H. 1993. Direct measurement of moisture in skin by NIR spectroscopy. J Soc Cosmet Chem, 44, 249-261.

Matcher, S J, and Cooper, C E. 1994. Absolute quantification of deoxyhaemoglobin concentration in tissue near infrared spectroscopy. Phys Med Biol, 39, 1295-1312.

Matcher, S J, Cope, M, and Delpy, D T. 1994. Use of the water spectrum to quantify tissue chromophore concentration changes in near-infrared spectroscopy. Phys Med Biol, 39, 177-196. 
Matcher, S J, Cope, M, and Delpy, D T. 1997. In vivo measurements of the wavelength dependence of tissue-scattering coefficients between 760 and $900 \mathrm{~nm}$ measured with time-resolved spectroscopy. Appl Opt, 36(1), 386-396.

Mathur-De Vré, R. 1984. Biomedical implications of the relaxation behaviour of water related to NMR imaging. Brit $J$ Radiol, 57(683), 955-976.

Matsukawa, T, Kashimoto, S, Ozaki, M, Shindo, S, and Kumazawa, T. 1996. Temperatures measured by a deep body thermometer (Coretemp ${ }^{\circledR}$ ) compared with tissue temperatures measured at various depths using needles placed into the sole of the foot. Euro J Anaesthesiol, 13, 340-345.

Mazarakis, N D, Edwards, A D, and Mehmet, H. 1997. Apoptosis in neural development and disease. Arch Dis Child Fetal Neonatal Ed, 77, F165-F170.

McCabe, W C, Subramanian, S, and Fisher, H F. 1970. A near-infrared spectroscopic investigation of the effect of temperature on the structure of water. J Phys Chem, 74(25), 4360-4369.

Meek, J H, Elwell, C E, McCormick, D C, Edwards, A D, Townsend, J P, Stewart, A L, and Wyatt, J S. 1999. Abnormal cerebral haemodynamics in perinatally asphyxiated neonates related to outcome. Arch Dis Child Fetal Neonatal Ed, 81, F110-F115.

Mehmet, H, Yue, X, Squier, M V, Lorek, A, Cady, E, Penrice, J, Sarraf, C, Wylezinska, M, Kirkbride, V, Cooper, C E, Brown, G C, Wyatt, J S, Reynolds, E O, and Edwards, A D. 1994. Increased apoptosis in the cingulate sulcus of newborn piglets following transient hypoxia-ischaemia is related to the degree of high energy phosphate depletion during the insult. Neurosci Lett, 181(1-2), 121-125.

Mie, G. 1908. Beitrage zur Optik trüber Medien speziell kolloidaler Metallösungen. Ann Physik, 25, 377-445.

Mizushina, S, Shimizu, T, and Sugiura, T. 1992. Non-invasive thermometry with multifrequency microwave radiometry. Front Med Biol Eng, 4(2), 129-133.

Montreuil, J, and Nachman, M. 1991. Multiangle method for temperature measurement of biological tissues by microwave radiometry. IEEE Trans Microwave Theory Tech, 39(7), 1235-1238. 
Mourant, J R, Fuselier, T, Boyer, J, Johnson, T M, and Bigio, I J. 1997. Predictions and measurements of scattering and absorption over broad wavelength ranges in tissue phantoms. Appl Opt, 36(4), 949-957.

Mourant, J R, Freyer, J P, Hielscher, A H, Eick, A A, Shen, D, and Johnson, T M. 1998. Mechanisms to light scattering from biological cells relevant to noninvasive optical-tissue diagnostics. Appl Opt, 37(16), 3586-3593.

Mourant, J R, Canpolat, M, Brocker, C, Esponder-Ramos, O, Johnson, T M, Matanock, A, Stetter, K, and Freyer, J P. 2000. Light scattering from cells: the contribution of the nucleus and the effects of proliferative status. J Biomed Opt, 5(2), 131-137.

Muma, B K, Treloar, D J, Wurmlinger, K, Peterson, E, and Vitae, A. 1991. Comparison of rectal, axillary, and tympanic membrane temperatures in infants and young children. Ann Emerg Med, 20(1), 41-44.

Muravchick, S. 1983. Deep body thermometry during general anesthesia. Anesthesiology, 58(3), 271-275.

Nakai, T, Nishimura, G, Yamamoto, K, and Tamura, M. 1997. Expression of optical diffusion coefficient in high-absorption turbid media. Phys Med Biol, 42, 2541-2549.

Nau, W H, Roselli, R J, and F, Milam D. 1999. Measurement of thermal effects on the optical properties of prostate tissue at wavelengths of 1,064 and $633 \mathrm{~nm}$. Lasers Surg Med, 24, 38-47.

Nichols, M G, Hull, E L, and Foster, T H. 1997. Design and testing of a white-light, steady-state diffuse reflectance spectrometer for determination of optical properties of highly scattering systems. Appl Opt, 36(1), 93-104.

Nilsson, A M, Lucassen, G W, Verkruysse, W, Andersson-Engels, S, and van Gemert, M J. 1997. Changes in optical properties of human whole blood in vitro due to slow heating. Photochem Photobiol, 65(2), 366-373.

Nilsson, A M, Sturesson, C, Liu, D L, and Andersson-Engels, S. 1998. Changes in spectral shape of tissue optical properties in conjunction with laser-induced thermotherapy. Appl Opt, 37(7), 1256-1267.

Nobel, J J. 1992. Infrared ear thermometry. Pediatr Emerg Care, 8(1), 54-58. 
Nomura, F, Naruse, H, duPlessis, A, Hiramatsu, T, Forbess, J, Holtzman, D, Volpe, J J, Jonas, R, and Tsuji, M. 1996. Cerebral oxygenation measured by near infrared spectroscopy during cardiopulmonary bypass and deep hypothermic circulatory arrest in piglets. Ped Res, 40(6), 790-796.

Okada, E. 2002. Personal communication. Keio University, Japan.

Okada, E, Firbank, M, Schweiger, M, Arridge, S R, Cope, M, and Delpy, D T. 1997. Theoretical and experimental investigation of near-infrared light propagation in a model of the adult head. Appl Opt, 36, 21-31.

Otto, M. 1998. Chemometrics. Statistics and computer application in analytical chemistry. Weinheim: Wiley-VCH.

Palade, G E. 1972. Mitochondria. Baltimore: University Park Press. Chap. An electron microscopy study of the mitochondrial structure, pages $35-58$.

Patel, J, and Edwards, A D. 1997. Prediction of outcome after perinatal asphyxia. Curr Opin Pediatr, 9(2), 128-132.

Patel, J, Marks, K, Roberts, I, Azzopardi, D, and Edwards, A D. 1998. Measurement of cerebral blood flow in newborn infants using near infrared spectroscopy with indocyanine green. Pediatr Res, 43(1), 34-39.

Patterson, M S, Chance, B, and Wilson, B C. 1989. Time resolved reflectance of transmittance for the non-invasive measurement of tissue optical properties. Appl Opt, 28(12), 2331-2336.

Patterson, M S, Wilson, B C, and Wyman, D R. 1991a. The propagation of optical radiation in tissue I. Models of radiation transport and their application. Lasers Med Sci, 6, 155-168.

Patterson, M S, Wilson, B C, and Wyman, D R. 1991b. The propagation of optical radiation in tissue II. Optical properties of tissue and resulting fluence distributions. Lasers Med Sci, 6, 379-390.

Patterson, M S, Andersson-Engels, S, Wilson, B C, and Osei, E K. 1995. Absorption spectroscopy in tissue-simulating materials: a theoretical and experimental study of photon paths. Appl Opt, 34(1), 22-30. 
Pegau, W S, and Zaneveld, J R. 1994. Temperature dependence of pure water in the visible portion of the spectrum. Pages 597-604 of: Ackleson, S (ed), Ocean Optics XII. Proc SPIE, vol. 2258.

Pegau, W S, Gray, D, and Zaneveld, J R. 1997. Absorption and attenuation of visible and near-infrared light in water: dependence on temperature and salinity. Appl Opt, 36(24), 6035-6046.

Penrice, J, Lorek, A, Cady, E B, Amess, P N, Wylezinska, M, Cooper, C E, D’Souza, P, Brown, G C, Kirkbride, V, Edwards, A D, Wyatt, J S, and Reynolds, E O. 1997. Proton magnetic resonance spectroscopy of the brain during acute hypoxia-ischemia and delayed cerebral energy failure in the newborn piglet. Pediatric Research, 41(6), 795-802.

Pimental, G C, and McClellan, A L. 1960. The hydrogen bond. Reinhold: Freeman.

Prahl, S A. 1988. Light transport in tissue. PhD Dissertation, University of Texas, Texas.

Press, W H, Teukolksy, S A, T, Vetterling W, and Flannery, B P. 1992. Numerical recipes in $C$. The art of scientific computing. Second edn. Cambridge: Cambridge University Press.

Rayleigh, Lord. 1871a. On the light from the sky, its polarisation and colour. Philos Mag, 41(273), 107-120.

Rayleigh, Lord. 1871b. On the light from the sky, its polarisation and colour. Philos Mag, 41(273), 274-279.

Rayleigh, Lord. 1881. On the electromagnetic theory of light. Philos Mag, 12(73), 81-101.

Robertson, N J, and Edwards, A D. 1998. Recent advances in developing neuroprotective strategies for perinatal asphyxia. Curr Opin Pediatr, 10(6), 575-580.

Robinson, J L, Seal, R F, Spady, D W, and Joffres, M R. 1998. Comparison of esophageal, rectal, axillary, bladder, tympanic and pulmonary artery temperatures in children. $J$ Pediatr, 133(4), 553-556.

Rolfe, P. 2002. In vivo near-infrared spectroscopy. Annu Rev Biomed Eng, 2, 715-754.

Ross, K F. 1967. Phase conrast and inteference microscopy for cell biologists. London: Edward Arnold. 
Rutishauser, S. 1994. Physiology and anatomy: a basis for nursing and health care. Edinburgh: Churchill Livingstone.

San Bagio, P L, Vitrano, E, Cupane, A, Madonia, F, and Palma, M U. 1977. Temperature induced difference spectra of oxy and deoxyhaemoglobin in the near ir, visible and Soret regions. Biochem Biophys Res Comm, 77(4), 1158-1165.

Sato, K T, Kane, N L, Soos, G, Gisolfi, C V, Kondo, N, and Sato, K. 1996. Reexamination of tympanic membrane temperature as a core temperature. J Appl Physiol, 80(4), 1233-1239.

Schmidt, F E, Fry, M E, Hillman, E M, and Hebden, J C. 2000. A 32-channel time-resolved instrument for medical optical tomography. Rev Sci Instrum, 71(1), 256-265.

Schneider, A S, Middaugh, C R, and Oldewurtel, M D. 1979. Role of bound water in biological membrane structure: Fluorescence and infrared studies. J Supramolec Struct, 10, 265-275.

Schultz, J W, and Hornig, D F. 1961. The effect of dissolved alkali halides on the Raman spectrum of water. J Phys Chem, 65, 2131-2138.

Schweiger, M, Arridge, S R, and Delpy, D T. 1993. Application of the finite element method for the forward and inverse models in optical tomography. J Math Imag Vision, 3, 263-83.

Schweiger, M, Arridge, S R, Hiraoka, M, and Delpy, D T. 1995. The finite element method for the propagation of light in scattering media: boundary and source conditions. Med Phys, 22(11), 1779-1792.

Seip, R, and Ebbini, E S. 1995. Noninvasive estimation of tissue temperature response to heating fields using diagnostic ultrasound. IEEE Trans Biomed Eng, 42(8), 828-839.

Sekar, T S, MacDonnell, K F, Namsirikul, P, and Herman, R S. 1980. Survival after prolonged submersion in cold water without neurologic sequelae. Report of two cases. Arch Intern Med, 140(6), 775-779.

Senior, W A, and Verrall, R E. 1969. Spectroscopic evidence for the mixture model in HOD solutions. J Phys Chem, 73(12), 4242.

Sfareni, R, Boff, A, Quaresima, V, and Ferrari, M. 1997. Near infrared absorption spectra of human deoxy- and oxyhaemoglobin in the temperature range $20-40{ }^{\circ} \mathrm{C}$. Biochim Biophys Acta, 1340, 165-169. 
Sganga, A, Wallace, R, Kiehl, E, Irving, T, and Witter, L. 2000. A comparison of four methods of normal newborn temperature measurement. MCN Am J Matern Child Nurs, 25(2), 76-9.

Shibasaki, M, Kondo, N, Tominaga, H, Aoki, K, Hasegawa, E, Idota, Y, and Moriwaki, T. 1998. Continuous measurement of tympanic temperature with a new infrared method using an optical fiber. $J$ Appl Physiol, 85(3), 921-926.

Shinozaki, T, Deane, R, and Perkins, F M. 1988. Infrared tympanic thermometer: evaluation of a new clinical thermometer. Crit Care Med, 16(2), 148-150.

Shiraki, K, Sagawa, S, Tajima, F, Yokota, A, Hashimoto, M, and Brengelmann, G L. 1988. Independence of brain and tympanic temperatures in an anaethetized human. $J A p p l$ Physiol, 65(1), 482-486.

Siebke, H, Rod, T, Breivik, H, and Link, B. 1975. Survival after 40 minutes; submersion without cerebral sequeae. Lancet, $7(1$ (7919)), 1275-1277.

Simbruner, G, Nanz, S, Fleishhacker, E, and Derganc, M. 1994. Brain temperature discriminates between neonates with damaged, hypoperfused and normal brains. $A m J$ Perinat, 11(2), 137-143.

Simbruner, G, Haberl, C, Harrison, V, Linley, L, and Willeitner, A E. 1999. Induced brain hypothermia in asphyxiated human newborn infants: a retrospective chart analysis of physiological and adverse effects. Intensive Care Med, 25(10), 1111-1117.

Simpson, R C, Kohl, M, Essenpreis, M, and Cope, M. 1998. Near-infrared optical properties of ex vivo human skin and subcutaneous tissues measured using the Monte Carlo inversion technique. Phys Med Biol, 43, 2465-2478.

Singer, B, and Lipton, B. 1975. Monitoring of core temperature measurement through the skin: a comparison with esophageal and tympanic temperatures. Bull NY Acad Med, $51,947-952$.

Skinner, M G, Everts, S, Reid, A D, Vitkin, I A, Lilge, L, and Sherar, M D. 2000. Changes in optical properties of ex vivo rat prostate due to heating. Phys Med Biol, 45, 13751386.

Solman, A J, and Dalton, J C. 1973. New thermometers for deep tissue temperature. Biomed Eng, 8(10), 432-435. 
Springett, R, Wylezinska, M, Cady, E B, Cope, M, and Delpy, D T. 2000. Oxygen dependency of cerebral oxidative phosphorylation in newborn piglets. J Cereb Blood Flow Metab, 20(2), 280-289.

Steinke, J M, and Shepherd, A P. 1988. Comparison of Mie theory and the light scattering of red blood cells. Appl Opt, 27(19), 4027-4033.

Stone, J G, Young, W L, Smith, C R, Soloman, R A, Wald, A, Ostapkovich, N, and Shrebnick, D B. 1995. Do standard monitoring sites reflect true brain temperature when profound hypothermia is rapidly induced and reversed? Anesthesiology, 82(2), $344-351$.

Terndrup, T E, Crofton, D J, Mortelliti, A J, Kelley, R, and Rajk, J. 1997. Estimation of contact tympanic membrane temperature with a noncontact infrared thermometer. Ann Emerg Med, 30(2), 171-175.

Thoresen, M. 1999. Cooling the asphyxiated brain - ready for clinical trials? Eur $J$ Pediatr, 158(Suppl 1), S5-S8.

Thoresen, M, Penrice, J, Lorek, A, Cady, E B, Wylezinska, M, Kirkbride, V, Cooper, C E, Brown, G C, Edwards, A D, Wyatt, J S, and Reynolds, E O. 1995. Mild hypothermia after severe transient hypoxia-ischemia ameliorates delayed cerebral energy failure in the newborn piglet. Ped Res, 37(5), 1-4.

Togawa, T. 1979. Non-invasive physiological measurements. Vol. 1. London: Academic Press. edited by Rolfe P. Chap. 11, pages 261-277.

Togawa, T. 1985. Body temperature measurement. Clin Phys Physiol Meas, 6(2), 83-108.

Togawa, T, Nemoto, T, Yamazaki, T, and Kobayashi, T. 1976. A modifled internal temperature measurement device. Med Biol Eng, 14, 361-364.

Torricelli, A, Pifferi, A, Taroni, P, Giambattistelli, E, and Cubeddu, R. 2001. In vivo optical characterization of human tissues from $610-1010 \mathrm{~nm}$ by time-resolved reflectance spectroscopy. Phys Med Biol, 46, 2227-2237.

Troy, T L, and Thennadil, S N. 2001. Optical properties of human skin in the near infrared wavelength range of 1000 to $2200 \mathrm{~nm}$. J Biomed Opt, 6(2), 167-176.

Tsuji, T, Suma, K, Togawa, T, and Nemoto, T. 1976. Deep body temperature monitoring in cardiac surgery. Jap J Med Electron Biol Eng, 14, 220-224. 
van Beekvelt, M C, Borghuis, M S, van Engelen, B G, Wevers, R A, and Colier, W N. 2001. Adipose tissue thickness affects in vivo quantitative near-IR spectroscopy in human skeletal muscle. Clin Sci, 101, 21-28.

Van Bel, F, Zeeuwe, P E, Dorrepaal, C A, Benders, M J, Van de Bor, M, and Hardjowijono, R. 1996. Changes in cerebral hemodynamics and oxygenation during hypothermic cardiopulmonary bypass in neonates and infants. Biol Neonate, 70(3), 141-154.

van de Hulst, H C. 1980. Multiple light scattering tables, formulas and applications. New York: Academic Press.

van de Hulst, H C. 1981. Light scattering by small particles. New York: Dover Publications. van der Zee, P. 1993. Measurement and modelling of the optical properties of human tissue in the near infrared. $\mathrm{PhD}$, University College London, London.

van der Zee, P, and Delpy, D T. 1987. Simulations of the point spread function for light in tissue by Monte Carlo technique. Adv Exp Med Biol, 215, 179-191.

van der Zee, P, Cope, M, Arridge, S R, Essenpreis, M, Potter, L A, Edwards, A D, Wyatt, J S, McCormick, D C, Roth, S C, Reynolds, E O, and et al. 1992. Experimentally measured optical pathlengths for the adult head, calf and forearm and the head of the newborn infant as a function of inter optode spacing. Adv Exp Med Biol, 316, $143-53$.

van Staveren, H G, Moes, C J, van Marle, J, Prahl, S A, and Gemert, M J. 1991. Light scattering in Intralipid-10\% in the wavelength range of 400-1100 nanometers. Appl $O p t, 30,4507-4514$.

Vannucci, R C, and Perlman, J M. 1997. Interventions for perinatal hypoxic-ischemic encephalopathy. Pediatrics, 100(6), 1004-1014.

Wall, T T, and Hornig, D F. 1965. Raman intensities of HDO and structure in liquid water. J Chem Phys, 43(6), 2079-2087.

Walrafen, G E. 1967. Raman spectral studies of the effects of temperature on water structure. J Chem Phys, 47(1), 114-126.

Wang, L, and Jaques, S L. 1992. Monte Carlo modeling of light transport in multi-layered tissues in standard $C$. Anderson Cancer Center, University of Texas. 
Wang, Z, Noyszewski, E A, and Leigh, Jr., J S. 1990. In vivo measurement of deoxymyoglobin in human forearm. Mag Res Med, 14, 562-567.

Weissbluth, M. 1974. Hemoglobin: cooperativity and electronic properties. Berlin: Springer-Verlag.

Weng, L, Reid, J M, Shankar, P M, Soetanto, K, and Lu, X M. 1992. Nonuniform phase distribution in ultrasound speckle analysis - Part I: Background and experimental demonstration. IEEE Trans Ultrason Ferroelec Freq Contr, 39(3), 360-365.

White, D R, Widdowson, E M, Woodard, H Q, and Dickerson, J W. 1991. The composition of body tissues (II). Fetus to young adult. $B r J$ Radiol, 64(758), 149-159.

Wickramasinghe, Y A, Palmer, K S, Houston, R, Spencer, S A, Rolfe, P, Thorniley, M S, Oeseburg, B, and Colier, W. 1993. Effect of fetal hemoglobin on the determination of neonatal cerebral oxygenation by near-infrared spectroscopy. Ped Res, 34(1), 15-17.

Williams, G D, Dardzinski, B J, Buckalew, A R, and Smith, M B. 1997. Modest hypothermia preserves cerebral energy metabolism during hypoxia-ischemia and correlates with brain damage: a 31P nuclear magnetic resonance study in unanesthetized neonatal rats. Pediatr Res, 42(5), 700-708.

Wilson, B C, and Jacques, S L. 1990. Optical reflectance and transmittance of tissues: principals and applications. IEEE J Quant Elec, 26(12), 2186-2199.

Wolf, M, von Siebenthal, K, Keel, M, Dietz, V, Baenziger, O, and Bucher, H U. 2000. Tissue oxygen saturation measured by near-infrared spectrophotometry correlates with arterial oxygen saturation during induced oxygenation changes in neonates. Physiol Meas, 21, 481-491.

Woodard, H Q, and White, D R. 1986. The composition of body tissues. Br J Radiol, 59, 1209-1218.

Wray, S, Cope, M, Delpy, D T, Wyatt, J S, and Reynolds, E O. 1988. Characterization of the near infrared absorption spectra of cytochrome $a a_{3}$ and haemoglobin for the noninvasive monitoring of cerebral oxygenation. Biochim Biophys Acta, 933, 184-192.

Wyatt, J S. 1999. Personal communication. University College Hospital, London UK.

Wyatt, J S, Edwards, A D, Azzopardi, D, and Reynolds, E O. 1989. Magnetic resonance and near infrared spectroscopy for investigation of perinatal hypoxic-ischaemic brain injury. Arch Dis Child, 64(7), 953-963. 
Wyatt, J S, Cope, M, Delpy, D T, Richardson, C E, Edwards, A D, Wray, S, and Reynolds, E O. 1990. Quantitation of cerebral blood volume in human infants by near-infrared spectroscopy. J Appl Physiol, 68(3), 1086-1091.

$\mathrm{Xu}, \mathrm{X}$, Tikuisis, P, and Giesbrecht, G. 1999. A mathematical model for human brain cooling during cold-water near-drowning. J Appl Physiol, 86(1), 265-272.

Yeh, S, Khalil, O S, Hanna, C, Kantor, S, Wu, X, Jeng, T, and Bolt, R A. 2001. Temperature dependence of optical properties of in vivo human skin. SPIE Proc, 4350, 455-461.

Yetman, R J, Coody, D K, West, M S, Montgomery, D, and M, Brown. 1993. Comparison of temperature measurements by an aural infrared thermometer with measurements by traditional rectal and axillary techniques. J Pediatr, 122(5 (1)), 769-773.

Young, I, Hand, J, Oatridge, A, Prior, M, and Forse, G. 1994. Further observations on the measurement of tissue $\mathrm{T}_{1}$ to monitor temperature in vivo by MRI. Mag Res Med, 31, 342-345.

Young, I, Hajnal, J, Ling, J, and Hill-Cottingham, R. 1995. Modelling of tissue response to temperature as depicted in vivo with the water-peak phase-shift method. Proc RSNA, 81st Annual Meeting, Chicago.

Zhu, L, and Diao, C. 2001. Theoretical simulation of temperature distribution in the brain during mild hypothermia treatment for brain injury. Med Biol Eng Comp, 39, 681-687.

Zijp, J R, and ten Bosch, J J. 1998. Optical properties of bovine muscle tissue in vitro; a comparison of methods. Phys Med Biol, 43, 3065-3081.

Zuo, C S, Bowers, J L, Metz, K R, Nosaka, T, Sherry, A D, and Clouse, M E. 1996. TmDOTP5-: a substance for NMR temperature measurements in vivo. Mag Res Med, 36(6), 955-959. 